Supporting Information

\title{
Function of Fimsbactin B as an Acinetobacter-Selective Antibiotic Delivery Vehicle
}

\author{
Do Young Kim, Hak Joong Kim* \\ Department of Chemistry and Center for ProteoGenomics Research \\ Korea University, Seoul 02841, Republic of Korea
}

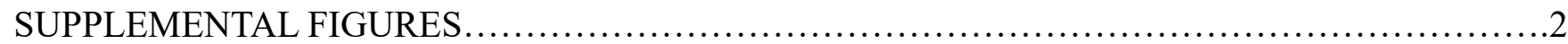

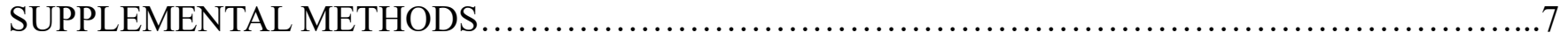

1. The Evaluation of the Antimicrobial Activity........................................

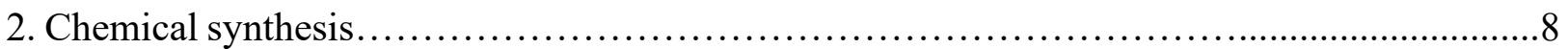

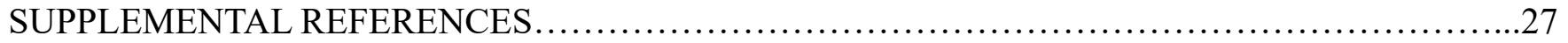

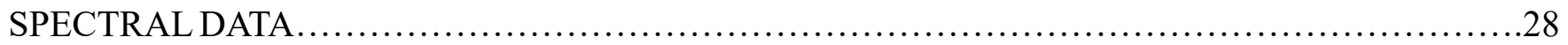




\section{SUPPLEMENTAL FIGURES}
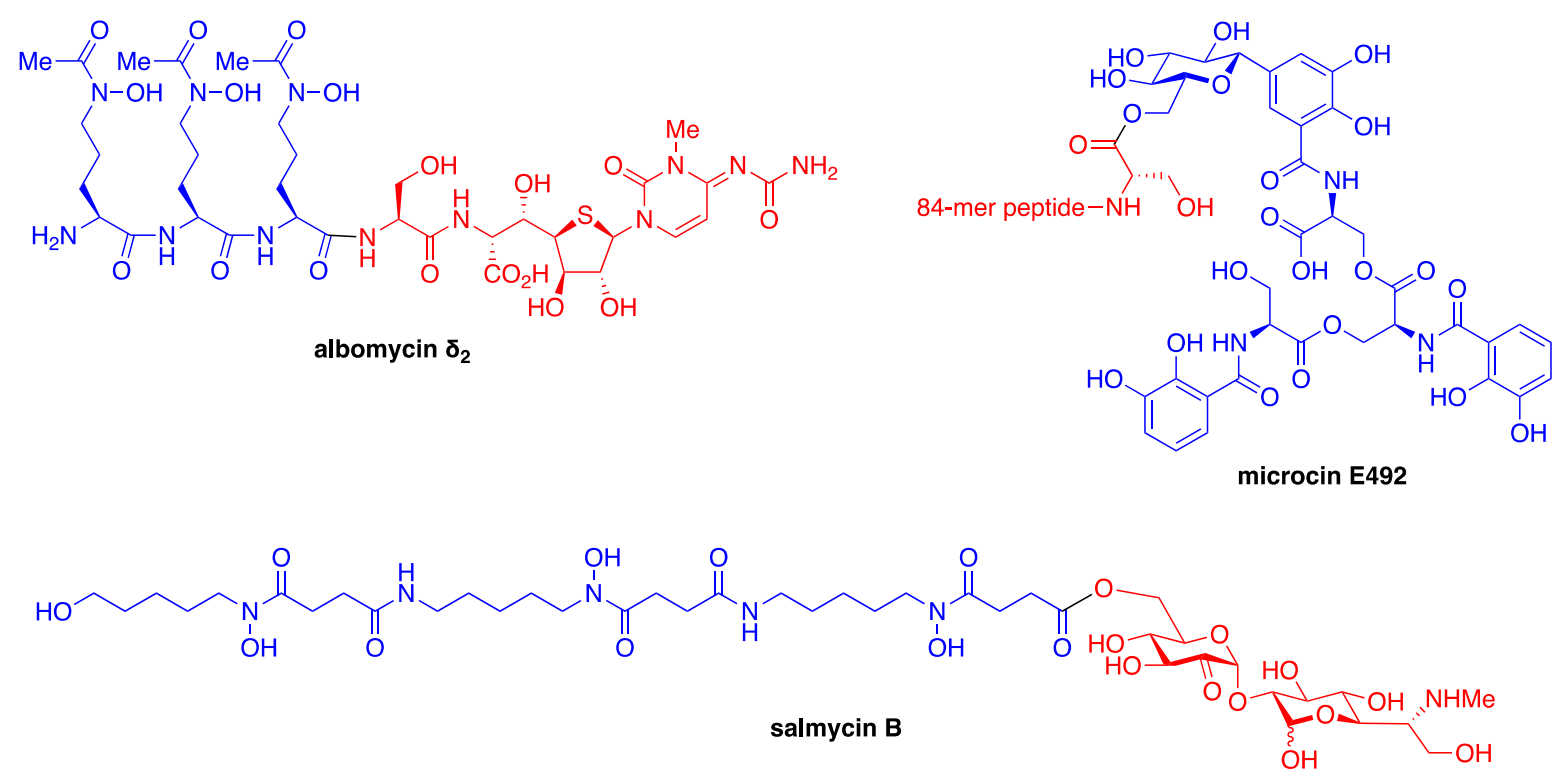

Figure S1. Structures of representative natural product sideromycins. Siderophore and antibiotic segments were colored in blue and red, respectively. 


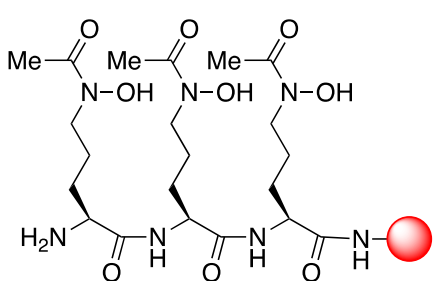

acyclic ferrichrome

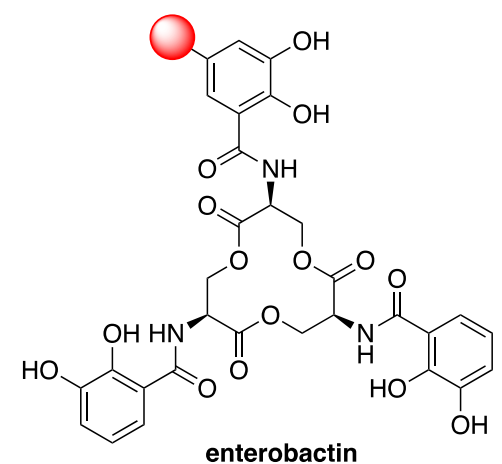<smiles>O=C(CCC(=O)N(O)CCCCCO)NCCCCCN(O)C(=O)CCC(=O)NCCCCCN(O)C(=O)CCC(=O)C1CCCC1</smiles><smiles>CC(=O)N(O)CCCCCNC(=O)CCC(=O)N(O)CCCCCNC(=O)CCC(=O)N(O)CCCCCNc1ccccc1</smiles>

desferrioxamine

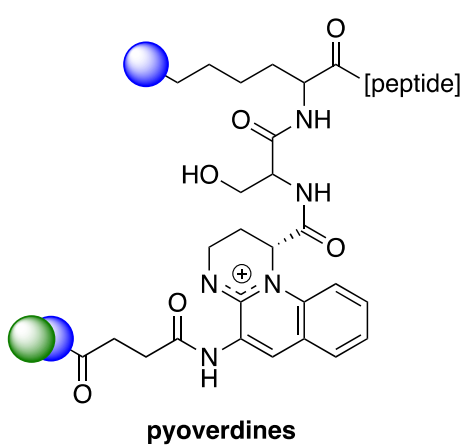<smiles>COc1ccc(O)c(C2=NC([C@H]3SC[C@@H](C(=O)O)C3CO)CS2)c1</smiles>

pyochelin

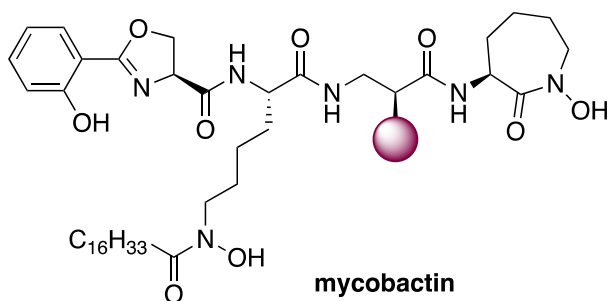

formation about the structure-activity relationship

Synthetic accessibility

Structural information of the cognate receptor

Natural sideromycin design

Figure S2. Examples of rational approaches employed for selecting the optimal antibiotic conjugation site during the design of novel sideromycins. 

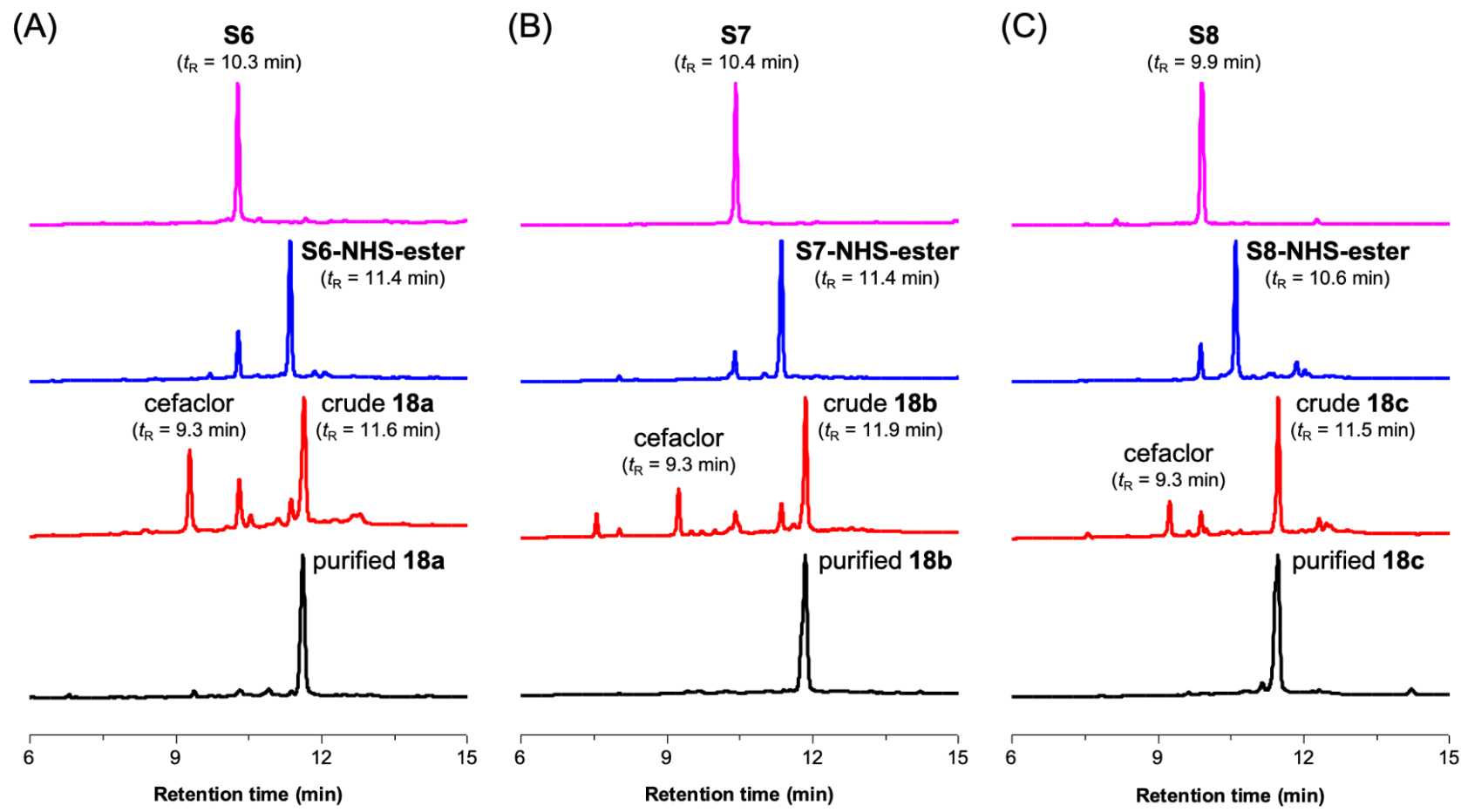

Figure S3. HPLC analysis-based monitoring of the synthetic processes for conjugation between each globally deprotected precursor (S6, S7, and S8) and cefaclor mediating the corresponding NHS-ester intermediates. Preparation of (A) 18a, (B) $\mathbf{1 8 b}$, and (C) $\mathbf{1 8 c}$ from S6, S7, and S8, respectively. All peaks were detected at $254 \mathrm{~nm}$. 


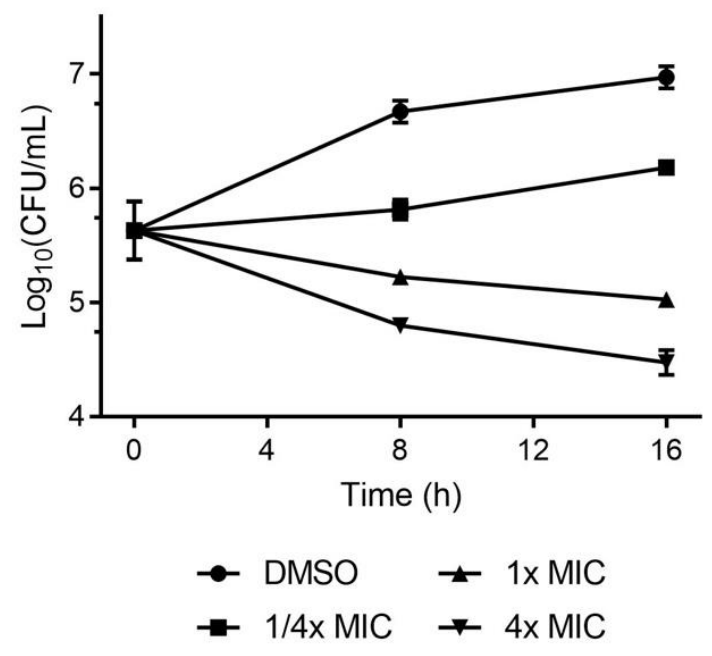

Figure S4. Time-kill experiment results using 18a showing the bactericidal activity of the molecule. The culture was grown in the CAMHB media containing $300 \mu \mathrm{M}$ DP at $37^{\circ} \mathrm{C}$. Error bars represent the standard deviations $(n=3)$. 


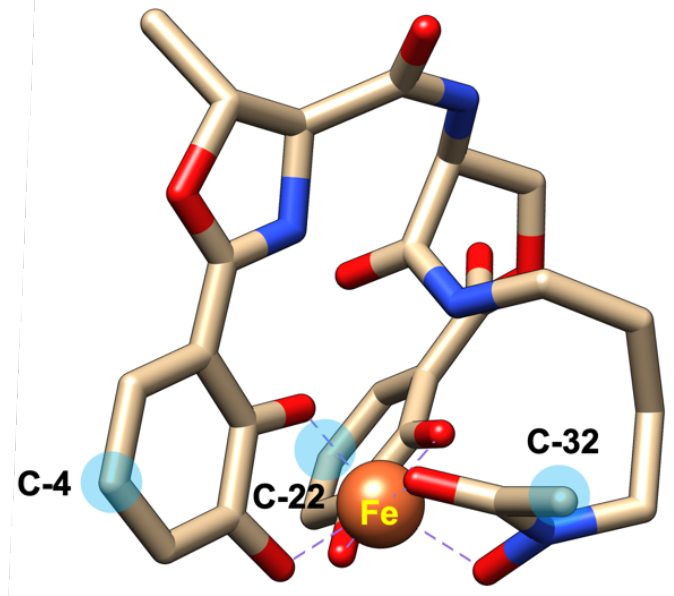

Figure S5. Iron-fimsbactin B complex model generated using CambridgeSoft Chem3D Pro version 12. Energy was minimized by molecular mechanics calculation. 


\section{SUPPLEMENTAL METHODS}

\section{The Evaluation of the Antimicrobial Activity}

\subsection{Strains and Materials}

The strains used in this study were obtained from the following sources: Acinetobacter baumannii ATCC 17978, A. baumannii ATCC 19606, and A. nosocomialis ATCC 17903 from the American Type Culture Collection (ATCC), Escherichia coli KCTC 1467 (K12), and Pseudomonas putida KCTC 1751 (equivalent to ATCC 12633) from Korean Collection for Type Cultures (KCTC). Each strain was maintained according to the instruction provided by the corresponding distributor. Both Luria-Bertani broth (LB broth, Miller, C/N 244620) and cation-adjusted Mueller-Hinton II broth (CAMHB, C/N 212322) were obtained from BD Difco (New Jersey, USA). WT strain was used in this study. Culture media were as follows: BBL Mueller-Hinton II broth Cation-Adjusted (CAMHB, Becton, Dickinson) media and $300 \mu \mathrm{M}$ 2,2'-dipyridyl (DP) to reduce iron availability (CAMHB$\mathrm{DP}_{300}$ ). Other reagents used in the biological experiments were obtained from Sigma-Aldrich (Michigan, USA).

\subsection{Procedure for the MIC Determination}

The MIC was determined according to the Clinical \& Laboratory Standard Institute (CLSI) guideline (M07, 11th edition). A frozen stock of the indicated bacterium was spread on the Luria-Bertani-agar (LB-agar) plate. After overnight incubation at 37 ${ }^{\circ} \mathrm{C}$, a single colony was picked to inoculate the LB broth. After overnight incubation at $37^{\circ} \mathrm{C}$, the culture solution was diluted by 100 -fold with CAMHB. After incubation at $37^{\circ} \mathrm{C}$ for $3 \mathrm{~h}$, this starter culture was diluted to the optical density at $600 \mathrm{~nm}$ (OD600) of 0.01, corresponding to ca. $5 \times 10^{5} \mathrm{CFU} / \mathrm{mL}$. In a 96-well microplate (Greiner Bio-One, C/N 655180, Kremsmünster, Austria), each well was loaded with $2 \mu \mathrm{L}$ solution of a chemical in DMSO, followed by treatment of $198 \mu \mathrm{L}$ starter culture prepared as described above. After covered with Breath-Easy ${ }^{\circledR}$ film (Diversified Biotech, C/N BEM-1), the microplate was incubated at $37^{\circ} \mathrm{C}$ for $24 \mathrm{~h}$ or $48 \mathrm{~h}$ before monitoring the cell growth inhibition to determine the MIC values. All experiments were conducted in duplicate or triplicate.

\subsection{Procedure for the time kill assay}

A frozen stock of the indicated bacterium was spread on the LB-agar plate. After overnight incubation at $37^{\circ} \mathrm{C}$, a single colony was picked to inoculate the LB broth. After overnight incubation at $37^{\circ} \mathrm{C}$, the culture solution was diluted by 100 -fold with CAMHB. After incubation at $37^{\circ} \mathrm{C}$ for $3 \mathrm{~h}$, this starter culture was diluted to the optical density at $600 \mathrm{~nm}$ of 0.01 , corresponding to ca. $5 \times 10^{5} \mathrm{CFU} / \mathrm{mL}$. Then, this culture solution aliquoted by $2,985 \mu \mathrm{L}$ and each sample was loaded with $15 \mu \mathrm{L}$ the indicated concentration of $\mathbf{1 8 a}$ solution in DMSO. Following the initiation of incubation at $37^{\circ} \mathrm{C}$, the culture was sampled at $0 \mathrm{~h}, 8 \mathrm{~h}$, 
and $16 \mathrm{~h}$. The CFU/mL of each sample was determined by overlaying the LB-agar plate with appropriate diluents. The resulting colonies were counted using ImageJ (U. S. National Institutes of Health, Bethesda, Maryland, USA), and the results were plotted using GraphPad Prism 7 (GraphPad software, San Diego, California, USA). This assay was conducted in triplicates.

\section{Chemical synthesis}

\subsection{Materials and general methods}

All reactions were conducted in an oven-dried glassware under inert atmospheric conditions with anhydrous solvents, unless otherwise noted. All reactions were monitored by analytical thin-layer chromatography (TLC) using a precoated silica aluminum plate with F254 indicators, and the product profiles were visualized by UV irradiation $(254 \mathrm{~nm})$ and staining with ninhydrin, potassium permanganate, and/or phosphomolybdic acid solution. The solvents including dichloromethane, tetrahydrofuran, ethyl ether, and toluene were dried by being passed through activated alumina column. Anhydrous methanol, dimethylformamide, acetonitrile, and other solvents were purchased from Sigma-Aldrich (Missouri, USA) or Alfa-Aesar (Massachusetts, USA). All other chemicals were purchased from Sigma-Aldrich, Alfa Aesar, TCI Chemicals (Japan), AK scientific (California, USA), or Daejung Chemicals \& Metals (Republic of Korea), and they were used as received unless otherwise noted.

The ${ }^{1} \mathrm{H}$ NMR and ${ }^{13} \mathrm{C}$ NMR data were recorded using Bruker Advance 500 (Bruker, Massachusetts, United States). Chemical shifts were reported in parts per million (ppm) relative to chloroform $\left({ }^{1} \mathrm{H}: 7.26 \mathrm{ppm},{ }^{13} \mathrm{C}: 77.16 \mathrm{ppm}\right)$, methanol $\left({ }^{1} \mathrm{H}: 3.31 \mathrm{ppm}\right.$, ${ }^{13} \mathrm{C}: 49.00 \mathrm{ppm}$ ), $\mathrm{D}_{2} \mathrm{O}\left({ }^{1} \mathrm{H}: 4.65 \mathrm{ppm}\right)$, dimethyl sulfoxide $\left({ }^{1} \mathrm{H}: 2.50 \mathrm{ppm},{ }^{13} \mathrm{C}: 39.52 \mathrm{ppm}\right.$ ), or tetramethylsilane (TMS, 0.00 $\mathrm{ppm}$ ), and the coupling constants were reported in Herz (Hz). High-resolution mass spectra were collected using Bruker Compact QTOF, where the electrospray ionization method was employed for ionization.

Both analytical and preparative high performance liquid chromatography (HPLC) experiments were conducted using a Thermo Ultimate 3000 equipped with a diode array detector (commonly, the absorbance at $215 \mathrm{~nm}, 254 \mathrm{~nm}$, and $280 \mathrm{~nm}$ was monitored). For analysis and purification, Acclaim $5 \mu \mathrm{m} \mathrm{C18} 120 \AA$ column (Thermo Fisher scientific, $150 \times 4.6 \mathrm{~mm}, \mathrm{C} / \mathrm{N}$ : 05941) and Luna $5 \mu \mathrm{m} \mathrm{C18(2)} 100 \AA$ column (Phenomenex, $250 \times 10 \mathrm{~mm}, \mathrm{P} / \mathrm{N}: 00 \mathrm{G}-4252-\mathrm{N} 0)$ were used, respectively.

\subsection{Synthetic procedures}

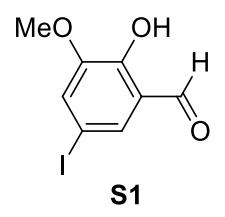

2-Hydroxy-5-iodo-3-methoxybenzaldehyde (S1) 
Compound $\mathbf{S 1}$ was synthesized from the $o$-vanillin as previously reported (Syn. Comm. 2008, 38, 3, 434).

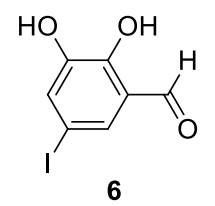

\section{2,3-Dihydroxy-5-iodobenzaldehyde (6)}

To a solution of compound $\mathbf{S 1}(15.7 \mathrm{~g}, 56.6 \mathrm{mmol})$ in dichloromethane $(250 \mathrm{~mL})$ was added boron tribromide $(10.7 \mathrm{~mL}, 113$. mmol) at $-78{ }^{\circ} \mathrm{C}$. The reaction mixture was stirred at room temperature for $30 \mathrm{~min}$ before quenched with iced water. The reaction mixture was then diluted with dichloromethane, washed with brine, and dried over anhydrous magnesium sulfate. After concentrated under reduced pressure, the crude residue was purified by flash column chromatography $\left(\mathrm{SiO}_{2}\right.$, hexanes:ethyl acetate $=9: 1)$ to afford the desired product $6(12.4 \mathrm{~g}, 47.0 \mathrm{mmol}, 83 \%$ yield $)$ as a white solid. ${ }^{1} \mathrm{H}$ NMR (500 $\left.\mathrm{MHz}, \mathrm{CDCl}_{3}\right) \delta 11.05(\mathrm{~s}, 1 \mathrm{H}), 9.81(\mathrm{~s}, 1 \mathrm{H}), 7.47(\mathrm{dd}, J=2.0,0.7 \mathrm{~Hz}, 1 \mathrm{H}), 7.46(\mathrm{~d}, J=2.0 \mathrm{~Hz}, 1 \mathrm{H}), 5.63(\mathrm{~s}, 1 \mathrm{H}) .{ }^{13} \mathrm{C} \mathrm{NMR}$ $\left(125 \mathrm{MHz}, \mathrm{CDCl}_{3}\right) \delta 195.6,148.4,146.0,132.7,130.0,122.1,80.7$. HR-MS (ESI-TOF) $m / z$ for $\left[\mathrm{C}_{7} \mathrm{H}_{4} \mathrm{IO}_{3}\right]^{-}\left([\mathrm{M}-\mathrm{H}]^{-}\right)$: calculated 262.9211, measured 262.9208.

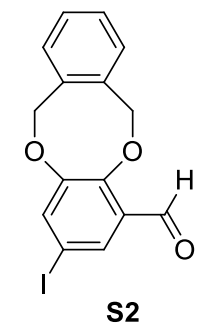

\section{3-Iodo-6,11-dihydrodibenzo[b,f][1,4]dioxocine-1-cabaldehyde (S2)}

To a solution of compound 6 (12.4 g, $47.0 \mathrm{mmol})$ in $N, N$-dimethylformamide (DMF, $50 \mathrm{~mL}$ ) was added potassium carbonate (19.4 g, $141 \mathrm{mmol})$ and $\alpha, \alpha^{\prime}$-dibromo-o-xylene $(14.7 \mathrm{~g}, 56.3 \mathrm{mmol})$. The reaction mixture was heated with an oil bath to $120^{\circ} \mathrm{C}$ and stirred for $5 \mathrm{~h}$. Then, the solvent was evaporated by vacuo. The reaction residue was then diluted with ethyl acetate, washed with brine, and dried over anhydrous magnesium sulfate. After concentrated under reduced pressure, the crude residue was purified by flash column chromatography $\left(\mathrm{SiO}_{2}\right.$, hexanes:ethyl acetate $\left.=19: 1\right)$ to afford the desired product $\mathbf{S 2}(10.5 \mathrm{~g}, 28.7$ mmol, $61 \%$ yield) as a white solid. ${ }^{1} \mathrm{H}$ NMR $\left(500 \mathrm{MHz}, \mathrm{CDCl}_{3}\right) \delta 10.30(\mathrm{~s}, 1 \mathrm{H}), 7.78(\mathrm{~d}, J=2.2 \mathrm{~Hz}, 1 \mathrm{H}), 7.58(\mathrm{~d}, J=2.3 \mathrm{~Hz}$, 1H), $7.33(\mathrm{dd}, J=5.7,3.3 \mathrm{~Hz}, 2 \mathrm{H}), 7.29(\mathrm{~s}, 1 \mathrm{H}), 7.17$ (dd, $J=5.2,3.6 \mathrm{~Hz}, 1 \mathrm{H}), 5.57(\mathrm{~s}, 2 \mathrm{H}), 5.39(\mathrm{~s}, 2 \mathrm{H}) .{ }^{13} \mathrm{C} \mathrm{NMR}(125 \mathrm{MHz}$, $\left.\mathrm{CDCl}_{3}\right) \delta 188.4,153.3,151.0,137.4,135.8,133.9,132.3,130.5,130.1,129.5,129.1,128.3,84.6,76.8,75.2$. 


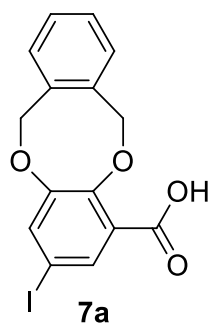

\section{3-Iodo-6,11-dihyrodibenzo[b,f][1,4]dioxocine-1-carboxylic acid (7a)}

To a solution of compound $\mathbf{S 2}(10.5 \mathrm{~g}, 28.7 \mathrm{mmol})$ in $t$-butanol $(280 \mathrm{~mL})$ was added 2-methyl-2-butene (30.4 $\mathrm{mL}, 287 \mathrm{mmol})$ at room temperature. This mixture was then treated with a solution of sodium chlorite $(7.78 \mathrm{~g}, 86.0 \mathrm{mmol})$ and monosodium phosphate $(10.3 \mathrm{~g}, 86.0 \mathrm{mmol})$ in $\mathrm{H}_{2} \mathrm{O}(20 \mathrm{~mL})$, and it was stirred for $5 \mathrm{~h}$. After removal of $t$-butanol under reduced pressure, the aqueous layer was basified with $10 \%$ sodium hydroxide solution and washed with dichloromethane. Then, the aqueous layer was acidified with $1 \mathrm{M}$ hydrochloric acid solution before extracted with ethyl acetate (three times). The combined organic layers were washed with brine and dried over anhydrous magnesium sulfate. After concentrated under reduced pressure, the desire product 7a (10.8 g, $28.3 \mathrm{mmol}, 99 \%$ yield) was afforded as a pure white solid without further purification. ${ }^{1} \mathrm{H}$ NMR (500 $\left.\mathrm{MHz}, \mathrm{CDCl}_{3}\right) \delta 8.14(\mathrm{~d}, J=2.3 \mathrm{~Hz}, 1 \mathrm{H}), 7.65-7.59(\mathrm{~m}, 1 \mathrm{H}), 7.35(\mathrm{ddd}, J=5.7,4.8,2.3 \mathrm{~Hz}, 2 \mathrm{H}), 7.33-7.31(\mathrm{~m}, 1 \mathrm{H}), 7.16$ (dd, $J=5.6,3.1 \mathrm{~Hz}, 1 \mathrm{H}), 5.70$ (s, 2H), 5.38 (s, 2H). ${ }^{13} \mathrm{C}$ NMR $\left(125 \mathrm{MHz}, \mathrm{CDCl}_{3}\right) \delta 164.1,150.2,149.9,137.1,137.0,136.1$, 132.2, 131.0, 130.2, 129.3, 128.1, 122.9, 85.2, 75.8. HR-MS (ESI-TOF) $\mathrm{m} / z$ for $\left[\mathrm{C}_{15} \mathrm{H}_{10} \mathrm{IO}_{4}\right]^{-}\left([\mathrm{M}-\mathrm{H}]^{-}\right)$: calculated 380.9629, measured 380.9628 .

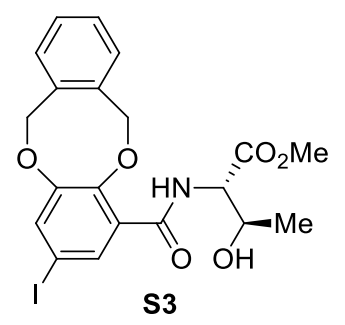

\section{Methyl (3-iodo-6,11-dihydrodibenzo[b,f][1,4]dioxocine-1-carbonyl)-L-threoninate (S3)}

To a solution of compound 7a $(10.8 \mathrm{~g}, 28.3 \mathrm{mmol})$ in DMF $(50 \mathrm{~mL})$ was added a solution of L-Thr- $\mathrm{OCH}_{3} \cdot \mathrm{HCl}(7.2 \mathrm{~g}, 42.3$ $\mathrm{mmol})$ and diisopropylethylamine $(14.8 \mathrm{~mL}, 84.8 \mathrm{mmol})$ pre-mixed in DMF $(20 \mathrm{~mL})$ at $0{ }^{\circ} \mathrm{C}$, followed by the addition of 1 hydroxybenzotriazole (HOBT, $7.64 \mathrm{~g}, 56.5 \mathrm{mmol}$ ) and 1-ethyl-3-(3-dimethylaminopropyl)carbodiimide (EDC, $10.8 \mathrm{~g}, 56.5$ mmol). The temperature was slowly elevated to room temperature, and the reaction mixture was stirred for $3 \mathrm{~h}$. The solvent was removed under reduced pressure, and the residue was diluted with ethyl acetate. The organic phase was washed with $1 \mathrm{~N}$ hydrochloric acid, saturated sodium bicarbonate solution, and brine, and then it was dried over anhydrous magnesium sulfate. The solvent was removed by vacuo to afford the desired product $\mathbf{S 3}$ (11.8 g, $23.7 \mathrm{mmol}, 84 \%$ yield). ${ }^{1} \mathrm{H}$ NMR $(500 \mathrm{MHz}$, $\left.\mathrm{CDCl}_{3}\right) \delta 8.77(\mathrm{~d}, J=8.4 \mathrm{~Hz}, 1 \mathrm{H}), 8.10(\mathrm{~d}, J=2.3 \mathrm{~Hz}, 1 \mathrm{H}), 7.51(\mathrm{~d}, J=2.3 \mathrm{~Hz}, 1 \mathrm{H}), 7.32-7.30(\mathrm{~m}, 3 \mathrm{H}), 7.15(\mathrm{dd}, J=6.7,2.6$ $\mathrm{Hz}, 1 \mathrm{H}), 5.72-5.54(\mathrm{~m}, 2 \mathrm{H}), 5.45-5.28(\mathrm{~m}, 2 \mathrm{H}), 4.82(\mathrm{dd}, J=8.5,2.4 \mathrm{~Hz}, 1 \mathrm{H}), 4.44(\mathrm{ddd}, J=10.8,5.6,3.1 \mathrm{~Hz}, 1 \mathrm{H}), 3.82$ 
(s, 3H), $2.15(\mathrm{~d}, J=4.9 \mathrm{~Hz}, 1 \mathrm{H}), 1.28$ (d, $J=6.4 \mathrm{~Hz}, 3 \mathrm{H}) .{ }^{13} \mathrm{C} \mathrm{NMR}\left(125 \mathrm{MHz}, \mathrm{CDCl}_{3}\right) \delta 171.8,164.1,150.7,149.4,136.1$, 135.7, 134.9, 133.4, 130.6, 129.5, 129.0, 128.1, 126.5, 84.9, 76.7, 75.2, 68.4, 57.8, 52.8, 20.2. HR-MS (ESI-TOF) $m / z$ for $\left[\mathrm{C}_{20} \mathrm{H}_{20} \mathrm{INNaO}_{6}\right]^{+}\left([\mathrm{M}+\mathrm{Na}]^{+}\right)$: calculated 520.0228, measured 520.0229.

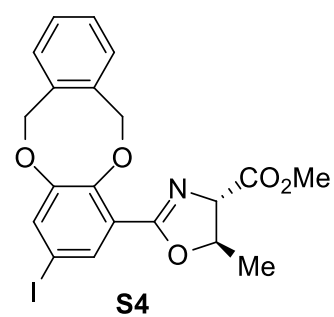

\section{Methyl (4S,5R)-2-(3-iodo-6,11-dihydrodibenzo[b,f] [1,4]dioxocin-1-yl)-5-methyl-4,5-dihydrooxazole-4-carboxylate (S4)}

This reaction was carried out in a flask fitted with a pressure-equalized addition funnel (containing a cotton plug and molecular sieves to function as a Soxhlet extractor) surmounted with a reflux condenser. A solution of compound S3 (11.8 g, $23.7 \mathrm{mmol}$ ) and bis(acetylacetonato)dioxomolybdenum(VI) $\left(\mathrm{MoO}_{2}(\mathrm{acac})_{2}, 774 \mathrm{mg}, 2.37 \mathrm{mmol}\right)$ in toluene $(250 \mathrm{~mL})$ was heated with an oil bath under azeotropic reflux. After $24 \mathrm{~h}$, the reaction mixture was cooled to the ambient temperature and concentrated under reduced pressure. The crude product was subjected to flash column chromatography $\left(\mathrm{SiO}_{2}\right.$, hexanes:ethyl acetate $\left.=9: 1\right)$ to yield the desired oxazoline ester $\mathbf{S 4}(8.87 \mathrm{~g}, 18.9 \mathrm{mmol}, 80 \%$ yield $) .1 \mathrm{H} \mathrm{NMR}\left(500 \mathrm{MHz}, \mathrm{CDCl}_{3}\right) \delta 7.73(\mathrm{~d}, J=2.2 \mathrm{~Hz}, 1 \mathrm{H}), 7.40$ $(\mathrm{d}, J=2.3 \mathrm{~Hz}, 1 \mathrm{H}), 7.28(\mathrm{dd}, J=6.2,2.9 \mathrm{~Hz}, 2 \mathrm{H}), 7.24-7.21(\mathrm{~m}, 1 \mathrm{H}), 7.18-7.14(\mathrm{~m}, 1 \mathrm{H}), 5.43$ (ddd, $J=32.0,15.5,11.0 \mathrm{~Hz}$, $4 \mathrm{H}), 4.97$ (dd, $J=7.1,6.3 \mathrm{~Hz}, 1 \mathrm{H}), 4.49$ (d, $J=7.2 \mathrm{~Hz}, 1 \mathrm{H}), 3.83(\mathrm{~s}, 3 \mathrm{H}), 1.55$ (d, $J=1.9 \mathrm{~Hz}, 3 \mathrm{H}) .{ }^{13} \mathrm{C} \mathrm{NMR}\left(125 \mathrm{MHz}, \mathrm{CDCl}_{3}\right)$ $\delta 171.5,162.9,152.0,149.2,135.8,134.7,133.5,133.5,129.7,128.9,128.7,128.6,123.7,85.4,78.8,75.9,75.2,74.8,52.7$, 21.1. HR-MS (ESI-TOF) $\mathrm{m} / z$ for $\left[\mathrm{C}_{20} \mathrm{H}_{19} \mathrm{INO}_{5}\right]^{+}\left([\mathrm{M}+\mathrm{H}]^{+}\right)$: calculated 480.0302 , measured 480.0306 .

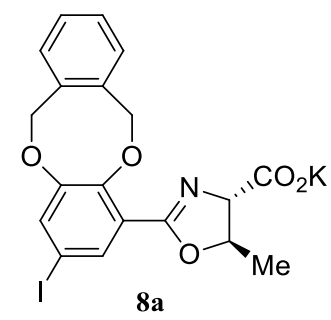

Potassium(4S,5R)-2-(3-iodo-6,11-dihydrodibenzo[b,f][1,4]dioxocin-1-yl)-5-methyl-4,5-dihydrooxazole-4-carboxylate (8a)

Ester S4 (8.87 g, $15.9 \mathrm{mmol})$ was dissolved in ethyl ether $(95 \mathrm{~mL})$ and treated with potassium trimethylsilanolate (2.68 g, 20.9 mmol) at room temperature. After stirred for $3 \mathrm{~h}$, the resulting solid was collected by filtration and washed with hexanes:ethyl acetate $=1: 1$ to afford the desired potassium carboxylate $8 \mathbf{a}(8.80 \mathrm{~g}, 17.5 \mathrm{mmol}, 98 \%$ yield $)$. The product was used in the next step without further purification. ${ }^{1} \mathrm{H} \mathrm{NMR}\left(500 \mathrm{MHz}, \mathrm{D}_{2} \mathrm{O}\right) \delta 7.73(\mathrm{~d}, J=2.2 \mathrm{~Hz}, 1 \mathrm{H}), 7.56(\mathrm{~d}, J=2.2 \mathrm{~Hz}, 1 \mathrm{H}), 7.38-7.26$ 
(m, 4H), $5.53-5.43(\mathrm{~m}, 4 \mathrm{H}), 4.88(\mathrm{p}, J=6.3 \mathrm{~Hz}, 1 \mathrm{H}), 4.30(\mathrm{~d}, J=6.7 \mathrm{~Hz}, 1 \mathrm{H}), 1.51(\mathrm{~d}, J=6.3 \mathrm{~Hz}, 3 \mathrm{H}) .{ }^{13} \mathrm{C} \mathrm{NMR}(125 \mathrm{MHz}$, $\left.\mathrm{D}_{2} \mathrm{O}\right) \delta 178.2,161.6,150.7,148.7,134.6,133.9,129.5,129.2,129.1,123.1,85.5,80.5,76.9,75.0,74.8,20.9$. HR-MS (ESITOF) $\mathrm{m} / z$ for $\left[\mathrm{C}_{19} \mathrm{H}_{17} \mathrm{INO}_{5}\right]^{+}\left([\mathrm{M}-\mathrm{K}+2 \mathrm{H}]^{+}\right)$: calculated 466.0146, measured 466.0148 .

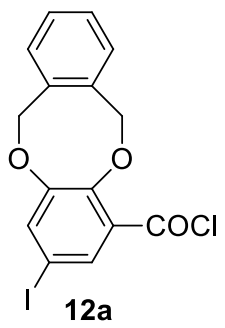

\section{3-Iodo-6,11-dihyrodibenzo[b,f][1,4]dioxocine-1-carbonyl chloride (12a)}

Carboxylic acid 7a $(1.00 \mathrm{~g}, 2.50 \mathrm{mmol})$ was treated with a thionyl chloride solution in dichloromethane $(1 \mathrm{M}, 50 \mathrm{~mL})$ at room temperature. After stirred for $14 \mathrm{~h}$ under reflux condition, heated with an oil bath, the reaction mixture was concentrated under reduced pressure to afford the desired carbonyl chloride 12a, which was used in the next step without further purification. ${ }^{1} \mathrm{H}$ NMR (500 MHz, DMSO-d $\left.{ }^{6}\right) \delta 7.52(\mathrm{~d}, J=2.2 \mathrm{~Hz}, 1 \mathrm{H}), 7.46(\mathrm{~d}, J=2.2 \mathrm{~Hz}, 1 \mathrm{H}), 7.33-7.26(\mathrm{~m}, 4 \mathrm{H}), 5.42(\mathrm{~d}, J=9.4 \mathrm{~Hz}, 4 \mathrm{H})$. ${ }^{13} \mathrm{C}$ NMR (125 MHz, DMSO-d $\left.{ }^{6}\right) \delta 165.5,151.4,148.4,135.3,135.1,133.1,132.6,129.5,129.2,128.9,85.9,74.8,74.3$.

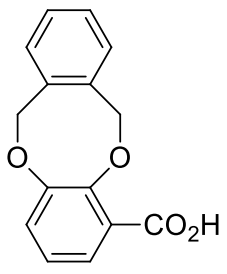

$7 \mathbf{b}$

\section{6,11-Dihydrodibenzo[b,f][1,4]dioxocine-1-carboxylic acid (7b)}

Compound $\mathbf{7 b}$ was prepared as previously reported (Bull. Kor. Chem. Soc. 2015, 36, 439).

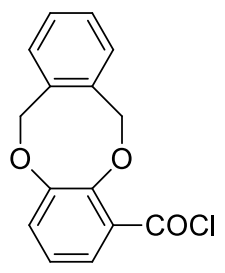




\section{6,11-Dihyrodibenzo[b,f][1,4]dioxocine-1-carbonyl chloride (12b)}

Synthesis of acid chloride 12b from carboxylic acid $7 \mathbf{b}(1.00 \mathrm{~g}, 3.64 \mathrm{mmol})$ was accomplished analogously to the transformation of 7a to 12a. ${ }^{1} \mathrm{H}$ NMR $\left(500 \mathrm{MHz}, \mathrm{DMSO}-\mathrm{d}^{6}\right) \delta 7.52(\mathrm{~d}, J=2.2 \mathrm{~Hz}, 1 \mathrm{H}), 7.46(\mathrm{~d}, J=2.2 \mathrm{~Hz}, 1 \mathrm{H}), 7.33-7.26$ $(\mathrm{m}, 5 \mathrm{H}), 5.42(\mathrm{~d}, J=9.4 \mathrm{~Hz}, 4 \mathrm{H}) .{ }^{13} \mathrm{C}$ NMR $\left(125 \mathrm{MHz}, \mathrm{DMSO}-\mathrm{d}^{6}\right) \delta 167.0,150.5,148.4,135.7,135.4,129.4,129.0,128.7$, $128.7,126.9,124.9,124.5,123.3,74.8,74.0$.

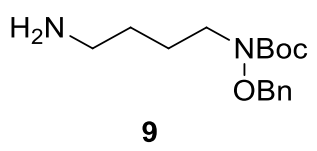

tert-Butyl (4-aminobutyl)(benzyloxy)carbamate (9)

Compound 9 was prepared as previously reported (ChemMedChem, 2020, 15, 23, 2269).

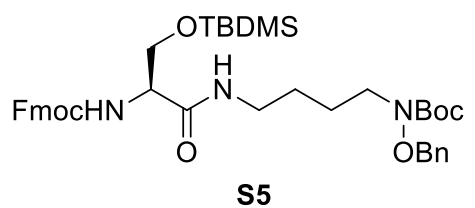

(S)-tert-Butyl (4-(2-((((9H-fluoren-9-yl)methoxy)carbonyl)amino)-3-((tert-butyldimethylsilyl)oxy)propanamido) butyl) (benzyloxy)carbamate (S5)

To a solution of amine 9 (10.0 g, $34.0 \mathrm{mmol})$ and Fmoc-Ser(TBDMS)-OH (19.5 g, $44.2 \mathrm{mmol})$ in DMF (80 mL) was added EDC $(13.0 \mathrm{~g}, 67.9 \mathrm{mmol})$ and $\mathrm{HOBt}(10.4 \mathrm{~g}, 67.9 \mathrm{mmol})$ at $0{ }^{\circ} \mathrm{C}$. After stirred for $3 \mathrm{~h}$ at room temperature, the reaction mixture was diluted with ethyl acetate and then poured into $1 \mathrm{~N} \mathrm{HCl}$ solution After collection of the organic layer, the aqueous layer was further extracted with ethyl acetate twice. The combined organic layers were washed with saturated sodium bicarbonate solution and brine, dried over anhydrous magnesium sulfate, and then concentrated under reduced pressure. The crude residue was subjected to flash column chromatography $\left(\mathrm{SiO}_{2}, 2 \%\right.$ methanol in dichloromethane) to afford the desired product $\mathbf{S 5}$ (17.6 g, $24.5 \mathrm{mmol}, 72 \%$ yield). ${ }^{1} \mathrm{H}$ NMR $\left(500 \mathrm{MHz}, \mathrm{CDCl}_{3}\right) \delta 7.77(\mathrm{t}, J=7.1 \mathrm{~Hz}, 2 \mathrm{H}), 7.60$ (t, $\left.J=12.1 \mathrm{~Hz}, 2 \mathrm{H}\right), 7.44-7.29$ (m, 9H), $6.52(\mathrm{~s}, 1 \mathrm{H}), 5.71(\mathrm{~s}, 1 \mathrm{H}), 4.81(\mathrm{~s}, 2 \mathrm{H}), 4.44-4.37(\mathrm{~m}, 2 \mathrm{H}), 4.22(\mathrm{t}, J=7.1 \mathrm{~Hz}, 1 \mathrm{H}), 4.12(\mathrm{q}, J=7.1 \mathrm{~Hz}, 1 \mathrm{H}), 4.07-3.97$ $(\mathrm{m}, 1 \mathrm{H}), 3.60(\mathrm{t}, J=8.6 \mathrm{~Hz}, 1 \mathrm{H}), 3.42(\mathrm{t}, J=6.9 \mathrm{~Hz}, 2 \mathrm{H}), 3.34-3.20(\mathrm{~m}, 2 \mathrm{H}), 1.61(\mathrm{dd}, J=14.8,7.2 \mathrm{~Hz}, 4 \mathrm{H}), 1.54-1.48(\mathrm{~m}$, $9 \mathrm{H}), 0.89$ (s, 9H), 0.08 (d, $J=6.5 \mathrm{~Hz}, 6 \mathrm{H}) .{ }^{13} \mathrm{C} \mathrm{NMR}\left(125 \mathrm{MHz}, \mathrm{CDCl}_{3}\right) \delta 170.1,156.7,144.0,143.8,141.5,135.7,129.6$, 128.7, 128.6, 127.9, 127.2, 125.2, 120.1, 81.5, 67.2, 63.3, 60.5, 55.8, 53.6, 49.1, 47.3, 39.5, 28.5, 26.8, 25.9, 24.7, 21.2, 18.3, 14.3, -5.3, -5.4. HR-MS (ESI-TOF) $\mathrm{m} / z$ for $\left[\mathrm{C}_{40} \mathrm{H}_{55} \mathrm{~N}_{3} \mathrm{NaO}_{7} \mathrm{Si}\right]^{+}\left([\mathrm{M}+\mathrm{Na}]^{+}\right)$: calculated 740.3701, measured 740.3705 . 


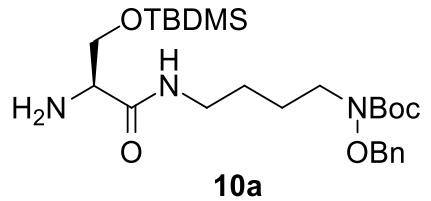

(S)-tert-Butyl (4-(2-amino-3-((tert-butyldimethylsilyl)oxy)propanamido)butyl)(benzyloxy)carbamate(10a)

A solution of compound $\mathbf{S 5}(17.6 \mathrm{~g}, 24.5 \mathrm{mmol}$ in dichloromethane $(80 \mathrm{~mL})$ was treated with diethylamine $(40 \mathrm{~mL})$ at room temperature. After stirred for $6 \mathrm{~h}$, the reaction mixture was concentrated under reduced pressure. The crude product was then subjected to flash column chromatography $\left(\mathrm{SiO}_{2}, 5 \%\right.$ methanol in dichloromethane) to afford the desired product $\mathbf{1 0 a}$ (14.8 $\mathrm{g}$, $23.6 \mathrm{mmol}, 97 \%$ yield). ${ }^{1} \mathrm{H}$ NMR $\left(500 \mathrm{MHz} \mathrm{CDCl}_{3}\right) \delta 7.43-7.32(\mathrm{~m}, 5 \mathrm{H}), 4.81(\mathrm{~s}, 2 \mathrm{H}), 3.77$ (ddd, J=16.0, $\left.9.8,5.4 \mathrm{~Hz}, 2 \mathrm{H}\right)$, $3.44-3.39(\mathrm{~m}, 3 \mathrm{H}), 3.23(\mathrm{dd}, J=13.3,6.9 \mathrm{~Hz}, 2 \mathrm{H}), 1.65-1.58(\mathrm{~m}, 4 \mathrm{H}), 1.50(\mathrm{~s}, 9 \mathrm{H}), 0.88(\mathrm{~s}, 9 \mathrm{H}), 0.05(\mathrm{~d}, J=4.4 \mathrm{~Hz}, 6 \mathrm{H})$. ${ }^{13} \mathrm{C} \mathrm{NMR}\left(125 \mathrm{MHz}, \mathrm{CDCl}_{3}\right) \delta 172.9,156.6,135.7,129.5,128.6,128.5,81.4,65.4,56.8,49.2,38.8,28.4,26.9,25.9,24.6$, 18.3, -5.3, -5.4. HR-MS (ESI-TOF) $m / z$ for $\left[\mathrm{C}_{25} \mathrm{H}_{46} \mathrm{~N}_{3} \mathrm{O}_{5} \mathrm{Si}\right]^{+}\left([\mathrm{M}+\mathrm{H}]^{+}\right)$: calculated 496.3201, measured 496.3204 .

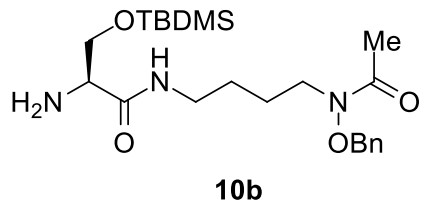

(S)-2-Amino-N-(4-(N-(benzyloxy)acetamido)butyl)-3-((tert-butyldimethylsilyl)oxy)propenamide (10b)

Compound 10b was prepared as previously reported (Bull. Kor. Chem. Soc. 2015, 36, 439).

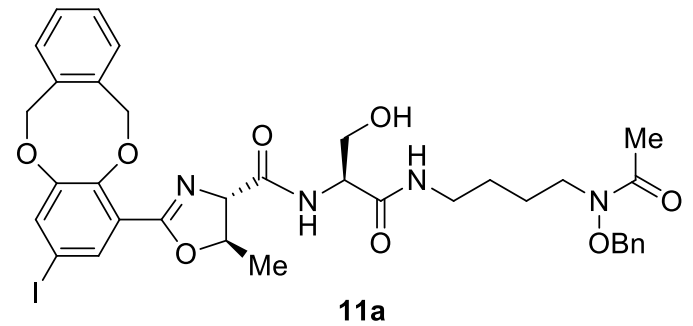

(4S,5R)-N-((S)-1-((4-(N-(Benzyloxy)acetamido)butyl)amino)-3-hydroxy-1-oxopropan-2-yl)-2-(3-iodo-6,11dihydrodibenzo[b,f][1,4] dioxocin-1-yl)-5-methyl-4,5-dihydrooxazole-4-carboxamide (11a)

To a solution of amine $\mathbf{1 0 b}(8.35 \mathrm{~g}, 19.1 \mathrm{mmol})$ in DMF $(50 \mathrm{~mL})$ was added potassium carboxylate $\mathbf{8 a}(8.00 \mathrm{~g}, 15.9 \mathrm{mmol})$, $\operatorname{EDC}(4.87 \mathrm{~g}, 31.8 \mathrm{mmol})$ and $\mathrm{HOBt}(4.87 \mathrm{~g}, 31.8 \mathrm{mmol})$ at $0^{\circ} \mathrm{C}$. After the reaction mixture was stirred at room temperature for $6 \mathrm{~h}$, the solvent was removed by vacuo. The resulting residue was then diluted with ethyl acetate, washed with brine, and dried over anhydrous magnesium sulfate. After concentrated under reduced pressure, the crude residue was purified by flash column chromatography $\left(\mathrm{SiO}_{2}, 1 \%\right.$ methanol in ethyl acetate) to afford the crude silyl protected intermediate (11.3 g, 12.7 
mmol, crude $80 \%$ yield), which was directly subjected to the deprotection reaction. This intermediate was then dissolved in tetrahydrofuran (THF, $60 \mathrm{~mL}$ ) and treated with tetrabutylammonium fluoride (TBAF, 1.0 M solution in THF, 19.2 mL, 19.2 $\mathrm{mmol}$ ) at $0{ }^{\circ} \mathrm{C}$. The reaction mixture was stirred for $1 \mathrm{~h}$ at room temperature, and then the solvent was removed by vacuo. The resulting crude residue was purified by flash column chromatography $\left(\mathrm{SiO}_{2}, 3 \%\right.$ methanol in ethyl acetate) to afford the desired product $11 \mathrm{a}\left(9.16 \mathrm{~g}, 11.9 \mathrm{mmol}\right.$, two-step $\underline{5874 \%}$ yield) as a white solid. ${ }^{1} \mathrm{H}$ NMR $\left(500 \mathrm{MHz}, \mathrm{CDCl}_{3}\right) \delta 7.76(\mathrm{~d}, J=7.6 \mathrm{~Hz}$, $1 \mathrm{H}), 7.72(\mathrm{~d}, J=2.3 \mathrm{~Hz}, 1 \mathrm{H}), 7.45(\mathrm{~d}, J=2.2 \mathrm{~Hz}, 1 \mathrm{H}), 7.40-7.35(\mathrm{~m}, 5 \mathrm{H}), 7.28(\mathrm{~d}, J=3.9 \mathrm{~Hz}, 2 \mathrm{H}), 7.19(\mathrm{dt}, J=7.7,2.7 \mathrm{~Hz}$, $1 \mathrm{H}), 6.79(\mathrm{t}, J=5.7 \mathrm{~Hz}, 1 \mathrm{H}), 5.65(\mathrm{~d}, J=13.2 \mathrm{~Hz}, 1 \mathrm{H}), 5.49(\mathrm{~d}, J=13.0 \mathrm{~Hz}, 1 \mathrm{H}), 5.46(\mathrm{~d}, J=13.2 \mathrm{~Hz}, 1 \mathrm{H}), 5.32(\mathrm{~d}, J=13.1$ $\mathrm{Hz}, 1 \mathrm{H}), 4.81(\mathrm{t}, J=3.1 \mathrm{~Hz}, 2 \mathrm{H}), 4.44(\mathrm{~d}, J=7.7 \mathrm{~Hz}, 1 \mathrm{H}), 4.40(\mathrm{ddd}, J=7.3,4.3,2.8 \mathrm{~Hz}, 1 \mathrm{H}), 4.17$ (ddd, $J=11.5,3.8,2.8 \mathrm{~Hz}$, 1H), 3.62 (ddd, $J=11.5,9.3,4.4 \mathrm{~Hz}, 3 \mathrm{H}), 3.41$ (dd, $J=8.8,3.2 \mathrm{~Hz}, 1 \mathrm{H}), 3.29$ (q, $J=6.6 \mathrm{~Hz}, 2 \mathrm{H}), 2.08$ (s, 3H), $1.69-1.65$ (m, $2 \mathrm{H}), 1.58(\mathrm{~d}, J=6.2 \mathrm{~Hz}, 3 \mathrm{H}), 1.55-1.51(\mathrm{~m}, 2 \mathrm{H}) .{ }^{13} \mathrm{C} \mathrm{NMR}\left(125 \mathrm{MHz}, \mathrm{CDCl}_{3}\right) \delta 172.0,170.2,162.1,151.8,149.8,135.4$, 134.8, 133.8, 133.4, 129.1, 129.0, 128.9, 128.8, 128.7, 128.6, 122.9, 84.8, 78.9, 76.2, 75.8, 75.6, 75.2, 62.6, 54.2, 39.0, 26.3, 25.7, 24.1, 21.8, 20.4. HR-MS (ESI-TOF) $\mathrm{m} / \mathrm{z}$ for $\left[\mathrm{C}_{35} \mathrm{H}_{40} \mathrm{IN}_{4} \mathrm{O}_{8}\right]^{+}\left([\mathrm{M}+\mathrm{H}]^{+}\right)$: calculated 771.1885, measured 771.1890.

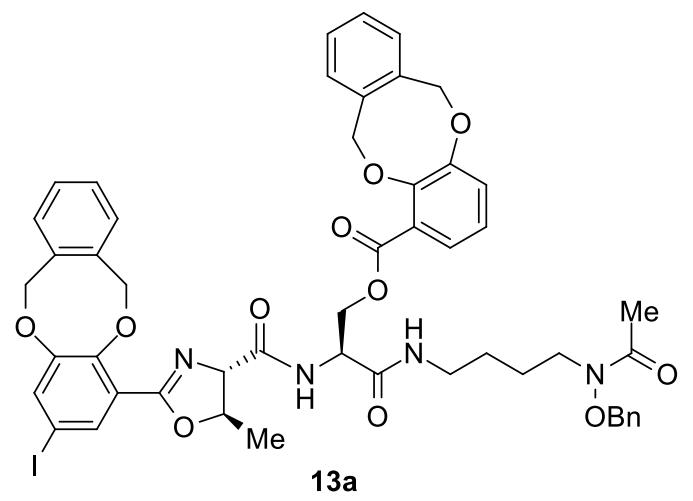

(S)-3-((4-(N-(Benzyloxy)acetamido)butyl)amino)-2-((4S,5R)-2-(3-iodo-6,11-dihydrodibenzo[b,f] [1,4]dioxocin-1-yl)-5methyl-4,5-dihydrooxazole-4-carboxamido)-3-oxopropyl 6,11-dihydrodibenzo[b,f][1,4]dioxocine-1-carboxylate (13a)

To a solution of compound 11a $(500 \mathrm{mg}, 0.649 \mathrm{mmol})$ and triethylamine $(271 \mu \mathrm{L}, 1.95 \mathrm{mmol})$ in dichloromethane $(4 \mathrm{~mL})$ was added a solution of $\mathbf{1 2 b}(357 \mathrm{mg}, 1.30 \mathrm{mmol})$ in dichloromethane $(2.5 \mathrm{~mL})$ dropwise at $0{ }^{\circ} \mathrm{C}$, followed by treatment of 4 dimethylaminopyridine (DMAP, $40.0 \mathrm{mg}, 0.325 \mathrm{mmol}$ ). After stirred for $1 \mathrm{~h}$ at room temperature, the reaction mixture was concentrated under reduced pressure. The crude residue was then purified by flash column chromatography $\left(\mathrm{SiO}_{2}, 3 \%\right.$ methanol in ethyl acetate) to afford the desired product 13a (576 mg, $0.571 \mathrm{mmol}, 88 \%$ yield) as a white solid. ${ }^{1} \mathrm{H}$ NMR (500 MHz, $\left.\mathrm{CDCl}_{3}\right) \delta 7.66(\mathrm{~d}, J=2.2 \mathrm{~Hz}, 1 \mathrm{H}), 7.63(\mathrm{~d}, J=7.4 \mathrm{~Hz}, 1 \mathrm{H}), 7.40-7.30(\mathrm{~m}, 8 \mathrm{H}), 7.25-7.20(\mathrm{~m}, 4 \mathrm{H}), 7.18-7.14(\mathrm{~m}, 3 \mathrm{H}), 7.11$ $(\mathrm{dd}, J=8.0,1.7 \mathrm{~Hz}, 1 \mathrm{H}), 6.81(\mathrm{t}, J=7.9 \mathrm{~Hz}, 1 \mathrm{H}), 6.73(\mathrm{t}, J=5.5 \mathrm{~Hz}, 1 \mathrm{H}), 5.56-5.26(\mathrm{~m}, 8 \mathrm{H}), 4.80(\mathrm{ddd}, J=10.5,8.7,6.0$ $\mathrm{Hz}, 2 \mathrm{H}), 4.76(\mathrm{~s}, 2 \mathrm{H}), 4.68$ (dd, $J=11.2,5.5 \mathrm{~Hz}, 1 \mathrm{H}), 4.48(\mathrm{dd}, J=11.2,6.0 \mathrm{~Hz}, 1 \mathrm{H}), 4.42(\mathrm{~d}, J=7.6 \mathrm{~Hz}, 1 \mathrm{H}), 3.57$ (s, 2H), $3.22(\mathrm{ddt}, J=19.6,13.4,6.6 \mathrm{~Hz}, 2 \mathrm{H}), 2.04(\mathrm{~d}, J=10.9 \mathrm{~Hz}, 3 \mathrm{H}), 1.59-1.53(\mathrm{~m}, 2 \mathrm{H}), 1.54(\mathrm{~d}, J=6.3 \mathrm{~Hz}, 3 \mathrm{H}), 1.43-1.37$ (m, 2H). ${ }^{13} \mathrm{C}$ NMR $\left(125 \mathrm{MHz}, \mathrm{CDCl}_{3}\right) \delta 172.5,171.9,168.3,165.4,162.5,151.9,151.4,150.2,149.8,135.6,135.5,135.4,134.9$, 134.4, 133.8, 133.5, 129.2, 129.2, 129.1, 128.9, 128.9, 128.8, 128.7, 126.2, 125.7, 124.2, 123.2, 85.0, 79.2, 76.4, 75.9, 75.9, 75.9, 75.3, 75.2, 64.0, 53.5, 52.2, 44.7, 39.4, 26.3, 24.4, 21.9, 20.6. HR-MS (ESI-TOF) $m / z$ for $\left[\mathrm{C}_{50} \mathrm{H}_{50} \mathrm{IN}_{4} \mathrm{O}_{11}\right]^{+}\left([\mathrm{M}+\mathrm{H}]^{+}\right)$: calculated 1009.2515, measured 1009.2517. 


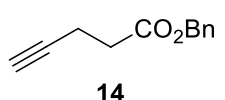

14

\section{Benzyl pent-4-ynoate (14)}

Compound 14 was prepared as previously reported (Org. Lett. 2016, 18, 5, 1020).

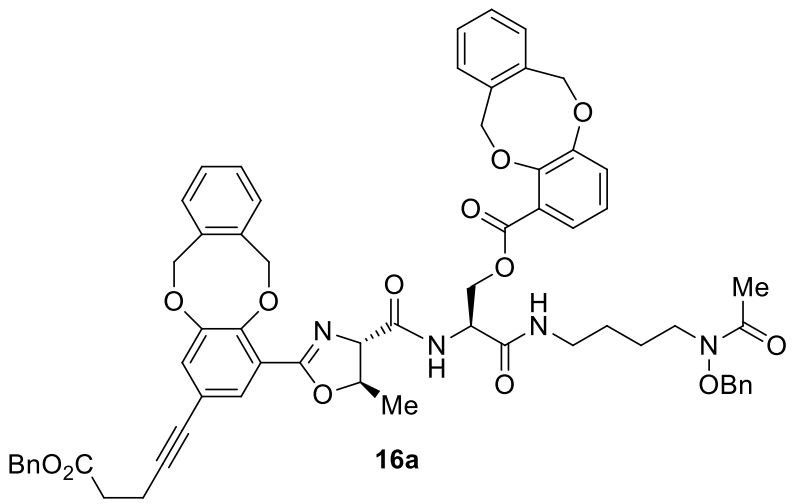

(S)-2-((4S,5R)-2-(3-(5-(Benzyloxy)-5-oxopent-1-yn-1-yl)-6,11-dihydrodibenzo[b,f][1,4]dioxocin-1-yl)-5-methyl-4,5-

dihydrooxazole-4-carboxamido)-3-((4-(N-(benzyloxy)acetamido)butyl)amino)-3-oxopropyl 6,11-dihydrodibenzo[b,f][1, 4]dioxocine-1-carboxylate (16a)

To a solution of compound 13a (100 mg, $0.0991 \mathrm{mmol})$, alkyne 14 (35.7 mg, 0.198mmol), and diisopropylethyl amine (86 $\mu \mathrm{L}$, $0.496 \mathrm{mmol})$ in degassed dimethylformamide $(2 \mathrm{~mL})$ was added tetrakis(triphenylphosphine)palladium $(0)(8.01 \mathrm{mg}, 6.94 \mu \mathrm{mol})$ and copper iodide $(1.89 \mathrm{mg}, 9.91 \mu \mathrm{mol})$, at which time the temperature was elevated with an oil bath to $100{ }^{\circ} \mathrm{C}$. After stirred for $30 \mathrm{~min}$, the reaction mixture was concentrated under reduced pressure. The crude residue was then purified by flash column chromatography $\left(\mathrm{SiO}_{2}, 3 \%\right.$ methanol in ethyl acetate) to afford the desired product 16a (71.0 mg, 0.0664 mmol, 67\%). ${ }^{1} \mathrm{H} \mathrm{NMR}$ $\left(500 \mathrm{MHz}, \mathrm{CDCl}_{3}\right) \delta 7.66(\mathrm{~d}, J=7.4 \mathrm{~Hz}, 1 \mathrm{H}), 7.42-7.08(\mathrm{~m}, 22 \mathrm{H}), 6.79(\mathrm{t}, J=7.9 \mathrm{~Hz}, 1 \mathrm{H}), 6.74(\mathrm{t}, J=4.5 \mathrm{~Hz} 1 \mathrm{H}), 5.60-$ $5.24(\mathrm{~m}, 8 \mathrm{H}), 5.15(\mathrm{~s}, 2 \mathrm{H}), 4.83-4.79(\mathrm{~m}, 1 \mathrm{H}), 4.80-4.76(\mathrm{~m}, 1 \mathrm{H}), 4.75(\mathrm{~s}, 2 \mathrm{H}), 4.66(\mathrm{dd}, J=11.2,5.5 \mathrm{~Hz}, 1 \mathrm{H}), 4.47(\mathrm{dd}, J$ $=11.2,6.1 \mathrm{~Hz}, 1 \mathrm{H}), 4.42(\mathrm{~d}, J=7.5 \mathrm{~Hz}, 1 \mathrm{H}), 3.56(\mathrm{~s}, 2 \mathrm{H}), 3.22(\mathrm{tdt}, J=20.3,13.4,6.8 \mathrm{~Hz}, 2 \mathrm{H}), 2.73-2.66(\mathrm{~m}, 2 \mathrm{H}), 2.66-$ $2.59(\mathrm{~m}, 2 \mathrm{H}), 2.04(\mathrm{~s}, 3 \mathrm{H}), 1.60-1.51(\mathrm{~m}, 2 \mathrm{H}), 1.53(\mathrm{~d}, J=6.2 \mathrm{~Hz}, 3 \mathrm{H}), 1.45-1.36(\mathrm{~m}, 2 \mathrm{H}) .{ }^{13} \mathrm{C} \mathrm{NMR}(125 \mathrm{MHz}, \mathrm{CDCl}) \delta$ $172.2,171.8,168.4,165.5,163.4,151.5,150.8,150.3,149.9,135.9,135.6,135.5,135.4,129.5,129.3,129.2,129.0,128.9$, 128.9, 128.8, 128.8, 128.8, 128.8, 128.7, 128.4, 128.3, 128.2, 126.3, 125.8, 124.2, 123.3, 121.3, 118.5, 88.1, 79.1, 76.4, 76.0, 76.0, 75.8, 75.5, 75.4, 66.6, 64.0, 52.2, 39.5, 33.7, 29.8, 26.3, 24.5, 22.0, 20.6, 15.4. HR-MS (ESI-TOF) $m / z$ for $\left[\mathrm{C}_{62} \mathrm{H}_{61} \mathrm{~N}_{4} \mathrm{O}_{13}\right]^{+}$ $\left([\mathrm{M}+\mathrm{H}]^{+}\right)$: calculated 1069.4230, measured 1069.4264 


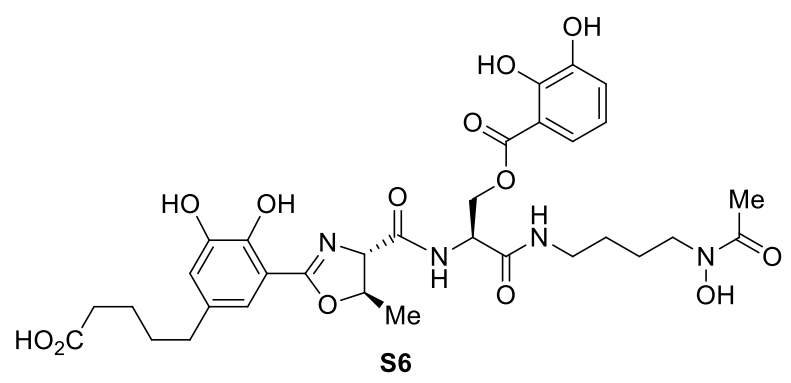

5-(3-((4S,5R)-4-(((S)-3-((2,3-Dihydroxybenzoyl)oxy)-1-((4-(N-hydroxyacetamido)butyl)amino)-1-oxopropan-2yl)carbamoyl)-5-methyl-4,5-dihydrooxazol-2-yl)-4,5-dihydroxyphenyl)pentanoicacid (S6)

To a solution of compound $\mathbf{1 6 a}(71.0 \mathrm{mg}, 0.0664 \mathrm{mmol})$ in methanol $(2 \mathrm{~mL})$ and ethyl acetate $(1 \mathrm{~mL})$ was added a palladium on activated carbon $(10 \% \mathrm{Pd} / \mathrm{C}, 70.6 \mathrm{mg}, 0.0664 \mathrm{mmol})$. This reaction mixture was then charged with hydrogen gas in a balloon. After stirred for $3 \mathrm{~h}$, the reaction mixture was filtered through a pad of celite to remove the catalyst and concentrated under reduced pressure to afford compound $\mathbf{S 6}(40.7 \mathrm{mg}, 0.0591 \mathrm{mmol} 89 \%$ yield $)$. This reaction progress was monitored by analytical HPLC (Acclaim $5 \mu \mathrm{m} \mathrm{C18} 120 \AA$ column, DW/ACN = 95/5 to 20/80 over $15 \mathrm{~min}, 0.1 \%$ trifluoroacetic acid was added as an ion pairing agent, flow rate $=1.0 \mathrm{~min} / \mathrm{mL}, 1=254 \mathrm{~nm}), t_{\mathrm{R}}=10.3 \mathrm{~min}(\mathbf{S 6}), t_{\mathrm{R}}=13.7 \mathrm{~min}(\mathbf{1 6 a}) .{ }^{1} \mathrm{H} \mathrm{NMR}\left(500 \mathrm{MHz}, \mathrm{CD}{ }_{3} \mathrm{OD}\right)$ $\delta 7.20(\mathrm{~d}, J=7.8 \mathrm{~Hz}, 1 \mathrm{H}), 6.95(\mathrm{~s}, 1 \mathrm{H}), 6.93(\mathrm{~d}, J=7.8 \mathrm{~Hz}, 1 \mathrm{H}), 6.82(\mathrm{~s}, 1 \mathrm{H}), 6.57(\mathrm{t}, J=7.7 \mathrm{~Hz}, 1 \mathrm{H}), 4.85-4.82(\mathrm{~m}, 2 \mathrm{H})$, $4.73(\mathrm{~d}, J=7.5 \mathrm{~Hz}, 1 \mathrm{H}), 4.55(\mathrm{~d}, J=6.6 \mathrm{~Hz}, 2 \mathrm{H}), 3.59(\mathrm{t}, J=6.3 \mathrm{~Hz}, 2 \mathrm{H}), 3.26(b r \mathrm{~s}, 2 \mathrm{H}), 2.51(b r \mathrm{~s}, 2 \mathrm{H}), 2.30(b r \mathrm{~s}, 2 \mathrm{H}), 2.08$ $(\mathrm{s}, 3 \mathrm{H}), 1.61(b r \mathrm{~s}, 6 \mathrm{H}), 1.53(b r \mathrm{~s}, 2 \mathrm{H}), 1.52(\mathrm{~d}, J=6.0 \mathrm{~Hz}, 3 \mathrm{H}) .{ }^{13} \mathrm{C} \mathrm{NMR}\left(125 \mathrm{MHz}, \mathrm{CD}_{3} \mathrm{OD}\right) \delta 173.6,173.1,171.2,170.4$, 168.6, 151.2, 147.7, 147.0, 146.4, 134.2, 122.0, 121.4, 120.8, 120.2, 119.2, 113.4, 111.2, 80.3, 75.5, 65.3, 53.7, 49.9, 48.4, 40.2, 35.8, 32.1, 27.3, 25.7, 25.0, 21.3, 20.2. HR-MS (ESI-TOF) $\mathrm{m} / z$ for $\left[\mathrm{C}_{32} \mathrm{H}_{40} \mathrm{~N}_{4} \mathrm{NaO}_{13}\right]^{+}\left([\mathrm{M}+\mathrm{Na}]^{+}\right.$): calculated 711.2484, measured 711.2485 .

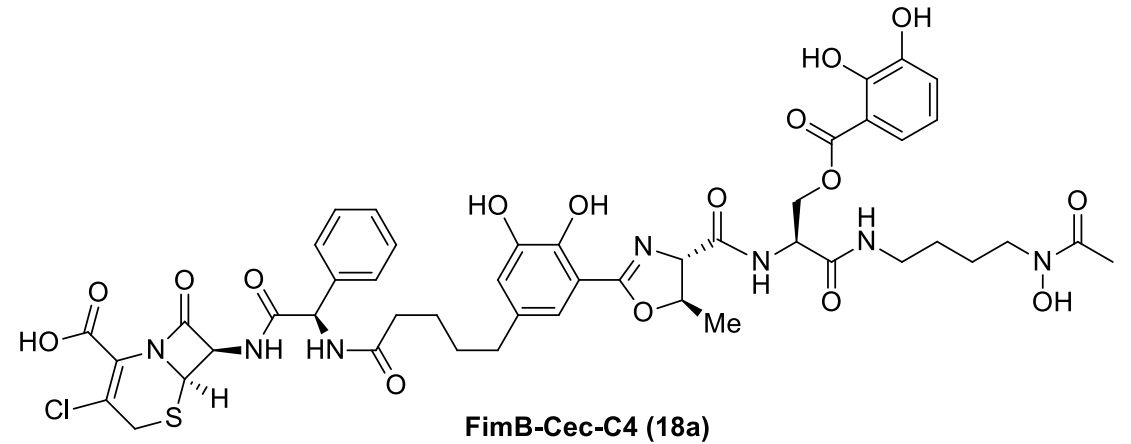

(6R)-3-Chloro-7-((S)-2-(5-(3-((4S,5R)-4-(((S)-3-((2,3-dihydroxybenzoyl)oxy)-1-((4-(N-hydroxyacetamido)butyl)amino)1-oxopropan-2-yl)carbamoyl)-5-methyl-4,5-dihydrooxazol-2-yl)-4,5-dihydroxyphenyl)pentanamido)-2phenylacetamido)-8-oxo-5-thia-1-azabicyclo[4.2.0]oct-2-ene-2-carboxylic acid (FimB-Cec-C4, 18a)

To a solution of compound $\mathbf{S 6}(13 \mathrm{mg}, 0.0189 \mathrm{mmol})$ in DMF $(300 \mu \mathrm{L})$ was added EDC (5.42 mg, $0.0283 \mathrm{mmol})$ and Nhydroxysuccinimide (NHS, $3.26 \mathrm{mg}, 0.0283 \mathrm{mmol}$ ) at room temperature. This reaction progress was monitored by analytical 
HPLC (Acclaim $5 \mu \mathrm{m}$ C18 $120 \AA$ column, DW/ACN = 95/5 to 20/80 over $15 \mathrm{~min}, 0.1 \%$ trifluoroacetic acid was added as an ion pairing agent, flow rate $=1.0 \mathrm{~min} / \mathrm{mL}, 1=254 \mathrm{~nm}), t_{\mathrm{R}}=10.3 \mathrm{~min}(\mathbf{S 6}), t_{\mathrm{R}}=11.4 \mathrm{~min}($ NHS-ester $), t_{\mathrm{R}}=11.6 \mathrm{~min}(\mathbf{1 8 a})$. After $6.5 \mathrm{~h}$ stirring, approximately $75 \%$ of compound $\mathbf{S 6}$ to the corresponding NHS-ester was converted, but further progression was not observed. To the resulting solution of NHS-ester in DMF was added a solution of cefaclor (10.4 $\mathrm{mg}, 0.0283 \mathrm{mmol})$ and diisopropylethyl amine $(13.2 \mu \mathrm{L}, 0.0755 \mathrm{mmol})$ in dimethyl sulfoxide $(300 \mu \mathrm{L})$ at room temperature. After $2 \mathrm{~h}$ stirring, approximately $92 \%$ conversion of the NHS ester intermediate to the corresponding cefaclor conjugate $\mathbf{1 8 a}$ was observed. The crude reaction mixture was purified by semi-preparative HPLC (Luna $5 \mu \mathrm{m} \mathrm{C18(2)} 100 \AA$ column, DW/ACN $=80 / 20$ to 65/35 over $50 \mathrm{~min}, 10 \mathrm{mM}$ ammonium bicarbonate was added as an ion pairing agent for $\mathrm{DW}$ and $\mathrm{pH}=7.2$, flow rate $=5.0 \mathrm{~mL} / \mathrm{min}$, $1=254 \mathrm{~nm}), t_{\mathrm{R}}=24.0 \mathrm{~min}(\mathbf{1 8 a})$. After lyophilization, the desired product 18a was afforded ( $3.50 \mathrm{mg}, 3.37 \mu \mathrm{mol}$, two-step $24 \%$ yield) as a white solid. ${ }^{1} \mathrm{H}$ NMR $\left(500 \mathrm{MHz}, \mathrm{CD}_{3} \mathrm{OD}\right) \delta 7.42(\mathrm{~d}, J=6.8 \mathrm{~Hz}, 2 \mathrm{H}), 7.37-7.26(\mathrm{~m}, 3 \mathrm{H}), 7.21(\mathrm{~d}, J=8.4 \mathrm{~Hz}, 1 \mathrm{H})$, $6.94(\mathrm{~d}, J=10.0 \mathrm{~Hz}, 2 \mathrm{H}), 6.81(\mathrm{~d}, J=1.8 \mathrm{~Hz}, 1 \mathrm{H}), 6.58(\mathrm{t}, J=8.0 \mathrm{~Hz}, 1 \mathrm{H}), 5.67(\mathrm{~d}, J=4.8 \mathrm{~Hz}, 1 \mathrm{H}), 5.53(\mathrm{~s}, 1 \mathrm{H}), 5.00(\mathrm{~d}, J=$ $4.8 \mathrm{~Hz}, 2 \mathrm{H}), 4.87-4.82(\mathrm{~m}, 2 \mathrm{H}), 4.73(\mathrm{dd}, J=11.3,4.3 \mathrm{~Hz}, 1 \mathrm{H}), 4.56(\mathrm{dd}, J=6.9,4.2 \mathrm{~Hz}, 2 \mathrm{H}), 3.72(\mathrm{~d}, J=17.6 \mathrm{~Hz}, 1 \mathrm{H}), 3.59$ $(\mathrm{t}, J=6.8 \mathrm{~Hz}, 2 \mathrm{H}), 3.30-3.20(\mathrm{~m}, 3 \mathrm{H}), 2.50(\mathrm{t}, J=7.0 \mathrm{~Hz}, 2 \mathrm{H}), 2.34-2.26(\mathrm{~m}, 2 \mathrm{H}), 2.08(\mathrm{~s}, 3 \mathrm{H}), 1.68-1.53(\mathrm{~m}, 8 \mathrm{H}), 1.52$ $(\mathrm{d}, J=6.3 \mathrm{~Hz}, 3 \mathrm{H}) .{ }^{13} \mathrm{C}$ NMR $\left(125 \mathrm{MHz}, \mathrm{DMSO}-\mathrm{d}^{6}\right) \delta 171.9,170.2,169.8,168.9,167.9,166.0,149.4,146.4,146.0,145.4$, 138.3, 132.4, 128.2, 127.5, 127.1, 127.0, 120.8, 119.7, 118.8, 117.0, 112.8, 109.7, 78.7, 73.4, 64.4, 58.1, 57.1, 55.5, 51.5, 48.6, 46.4, 38.5, 34.6, 34.1, 30.6, 26.1, 24.8, 23.7, 20.4. HR-MS (ESI-TOF) $m / z$ for $\left[\mathrm{C}_{47} \mathrm{H}_{53} \mathrm{ClN}_{7} \mathrm{O}_{16} \mathrm{~S}\right]^{+}\left([\mathrm{M}+\mathrm{H}]^{+}\right)$: calculated 1038.2953, measured 1038.2947.

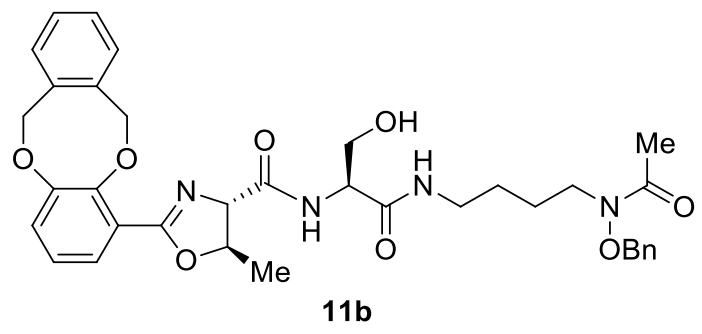

(4S,5R)-N-((S)-1-((4-(N-(Benzyloxy)acetamido)butyl)amino)-3-hydroxy-1-oxopropan-2-yl)-2-(6,11-dihydrodibenzo [b,f ][1,4]dioxocin-1-yl)-5-methyl-4,5-dihydrooxazole-4-carboxamide (11b)

Compound 11b was prepared as previously reported (Bull. Kor. Chem. Soc. 2015, 36, 439). ${ }^{1} \mathrm{H}$ NMR (500 MHz, $\left.\mathrm{CDCl}_{3}\right) \delta 7.83$ $-7.77(\mathrm{~m}, J=7.6 \mathrm{~Hz}, 1 \mathrm{H}), 7.43(\mathrm{dd}, J=7.8,1.6 \mathrm{~Hz}, 1 \mathrm{H}), 7.39-7.34(\mathrm{~m}, 5 \mathrm{H}), 7.30-7.22(\mathrm{~m}, 3 \mathrm{H}), 7.20-7.13(\mathrm{~m}, 2 \mathrm{H}), 6.96$ (t, $J=7.9 \mathrm{~Hz}, 1 \mathrm{H}), 6.89(\mathrm{~s}, 1 \mathrm{H}), 5.48(\mathrm{ddd}, J=77.0,68.2,13.2 \mathrm{~Hz}, 4 \mathrm{H}), 4.85-4.81(\mathrm{~m}, J=7.5,6.3 \mathrm{~Hz}, 1 \mathrm{H}), 4.80(\mathrm{~s}, 2 \mathrm{H}), 4.44$ $(\mathrm{d}, J=7.6 \mathrm{~Hz}, 1 \mathrm{H}), 4.41(\mathrm{~d}, J=2.8 \mathrm{~Hz}, 1 \mathrm{H}), 4.19-4.08(\mathrm{~m}, J=13.2 \mathrm{~Hz}, 1 \mathrm{H}), 3.67-3.58(\mathrm{~m}, 3 \mathrm{H}), 3.28(\mathrm{dd}, J=12.9,6.6 \mathrm{~Hz}$, $2 \mathrm{H}), 2.08(\mathrm{~s}, 3 \mathrm{H}), 1.70-1.63(\mathrm{~m}, 2 \mathrm{H}), 1.58(\mathrm{~d}, J=6.2 \mathrm{~Hz}, 3 \mathrm{H}), 1.52(\mathrm{dt}, J=13.9,6.9 \mathrm{~Hz}, 2 \mathrm{H})$. 


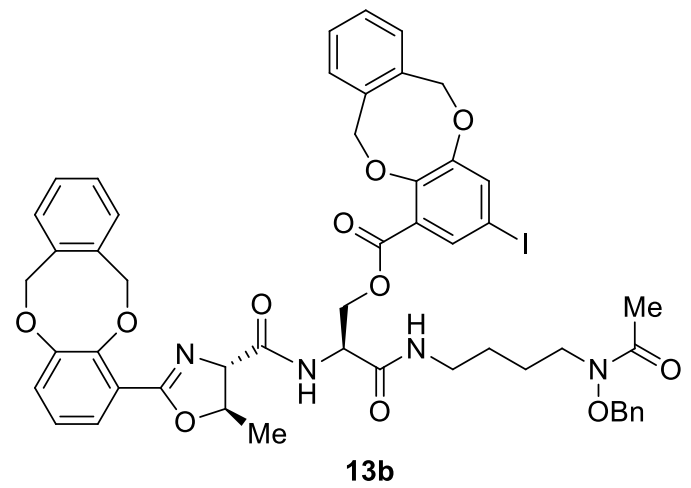

(S)-3-((4-(N-(Benzyloxy)acetamido)butyl)amino)-2-((4S,5R)-2-(6,11-dihydrodibenzo[b,f][1,4]dioxocin-1-yl)-5methyl-4,5-dihydrooxazole-4-carboxamido)-3-oxopropyl 3-iodo-6,11-dihydrodibenzo[b,f][1,4]dioxocine-1-carboxy late (13b)

To a solution of $11 \mathrm{~b}(500 \mathrm{mg}, 0.776 \mathrm{mmol})$ and triethylamine $(324 \mu \mathrm{L}, 2.33 \mathrm{mmol})$ in anhydrous dichloromethane $(3.00 \mathrm{~mL})$ was added a solution of 12a $(621 \mathrm{mg}, 1.55 \mathrm{mmol})$ in dichloromethane $(1.00 \mathrm{~mL})$ dropwise at $0{ }^{\circ} \mathrm{C}$, followed by treatment of 4 dimethylaminopyridine (DMAP, $47 \mathrm{mg}, 0.388 \mathrm{mmol}$ ). After $1 \mathrm{~h}$ stirring, the solvent was removed by vacuo. The crude residue was purified by flash column chromatography $\left(\mathrm{SiO}_{2}, 3 \%\right.$ methanol in ethyl acetate) to afford the desired product $\mathbf{1 3 b}(618 \mathrm{mg}$, $0.613 \mathrm{mmol}, 79 \%$ yield) as a white solid. ${ }^{1} \mathrm{H}$ NMR $\left(500 \mathrm{MHz}, \mathrm{CDCl}_{3}\right) \delta 7.61(\mathrm{~s}, 1 \mathrm{H}), 7.60(\mathrm{~d}, J=2.2 \mathrm{~Hz}, 1 \mathrm{H}), 7.40-7.33(\mathrm{~m}$, $7 \mathrm{H}), 7.26-7.13(\mathrm{~m}, 8 \mathrm{H}), 7.10(\mathrm{dd}, J=8.0,1.6 \mathrm{~Hz}, 1 \mathrm{H}), 6.87(\mathrm{t}, J=7.9 \mathrm{~Hz}, 1 \mathrm{H}), 6.79(\mathrm{~s}, 1 \mathrm{H}), 5.54-5.25(\mathrm{~m}, 8 \mathrm{H}), 4.86-4.82$ (m, 1H), $4.82-4.79(\mathrm{~m}, 1 \mathrm{H}), 4.77(\mathrm{~s}, 2 \mathrm{H}), 4.65(\mathrm{dd}, J=11.2,5.8 \mathrm{~Hz}, 1 \mathrm{H}), 4.49(\mathrm{dd}, J=11.2,5.5 \mathrm{~Hz}, 1 \mathrm{H}), 4.43(\mathrm{~d}, J=7.5 \mathrm{~Hz}$, $1 \mathrm{H}), 3.60(b r \mathrm{~s}, 2 \mathrm{H}), 3.31-3.19(\mathrm{~m}, 2 \mathrm{H}), 2.06(\mathrm{~s}, 3 \mathrm{H}), 1.63-1.58(\mathrm{~m}, 2 \mathrm{H}), 1.56(\mathrm{~d}, J=6.2 \mathrm{~Hz}, 3 \mathrm{H}), 1.45(\mathrm{dt}, J=13.4,6.6 \mathrm{~Hz}$, 2H). ${ }^{13} \mathrm{C}$ NMR (125 MHz, $\left.\mathrm{CDCl}_{3}\right) \delta 172.4,168.3,164.4,164.1,152.1,151.4,150.1,150.0,135.8,135.2,135.0,134.9,134.2$, 129.3, 129.2, 129.2, 129.1, 129.1, 129.0, 129.0, 128.9, 128.8, 128.6, 126.1, 125.3, 125.1, 123.3, 121.4, 85.0, 79.3, 76.5, 76.0, 76.0, 75.8, 75.3, 64.5, 52.2, 39.5, 26.3, 24.5, 22.0, 20.6. HR-MS (ESI-TOF) $m / z$ for $\left[\mathrm{C}_{50} \mathrm{H}_{50} \mathrm{IN}_{4} \mathrm{O}_{11}\right]^{+}\left([\mathrm{M}+\mathrm{H}]^{+}\right)$: calculated 1009.2515, measured 1009.2519.

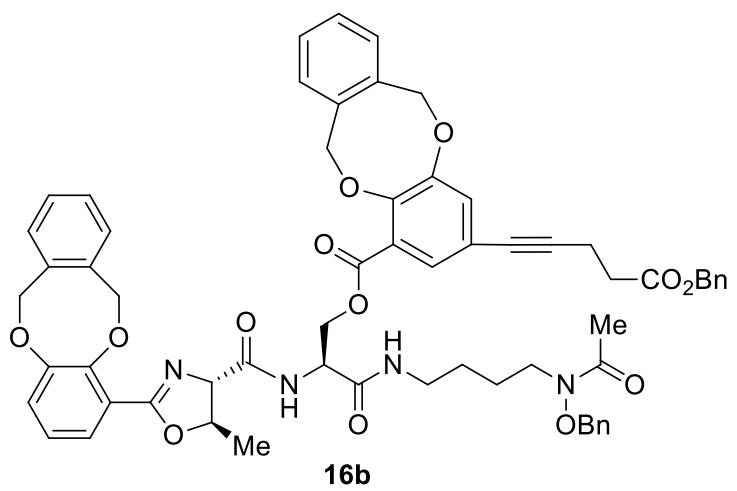

(S)-3-((4-(N-(Benzyloxy)acetamido)butyl)amino)-2-((4S,5R)-2-(6,11-dihydrodibenzo[b,f][1,4]dioxocin-1-yl)-5methyl-4,5-dihydrooxazole-4-carboxamido)-3-oxopropyl 3-(5-(benzyloxy)-5-oxopent-1-yn-1-yl)-6,11-dihydrodibenzo[b 


\section{,f][1,4]dioxocine-1-carboxylate (16b)}

To a solution of compound 13b (100 mg, $0.0991 \mathrm{mmol})$, alkyne 14 (35.7 mg, 0.198mmol), and DIPEA (86 $\mu \mathrm{L}, 0.496 \mathrm{mmol})$ in degassed dimethylformamide $(2 \mathrm{~mL})$ was added tetrakis(triphenylphosphine)palladium(0) $(8.01 \mathrm{mg}, 6.94 \mu \mathrm{mol})$ and copper iodide $(1.89 \mathrm{mg}, 9.91 \mu \mathrm{mol})$ at room temperature, after which time the temperature was elevated with an oil bath to $100{ }^{\circ} \mathrm{C}$. After $30 \mathrm{~min}$ stirring, the solvent was removed by vacuo. The resulting crude residue was purified by flash column chromatography ( $\mathrm{SiO}_{2}, 3 \%$ methanol in ethyl acetate) to afford the desired product $\mathbf{1 6 b}(65.7 \mathrm{mg}, 0.0615 \mathrm{mmol}, 62 \%)$. ${ }^{1} \mathrm{H}$ $\operatorname{NMR}\left(500 \mathrm{MHz}, \mathrm{CDCl}_{3}\right) \delta 7.70-7.64(\mathrm{~m}, 1 \mathrm{H}), 7.63(\mathrm{~d}, J=7.7 \mathrm{~Hz}, 1 \mathrm{H}), 7.39-7.28(\mathrm{~m}, 12 \mathrm{H}), 7.26-7.18(\mathrm{~m}, 4 \mathrm{H}), 7.18-$ $7.11(\mathrm{~m}, 3 \mathrm{H}), 7.11-7.06(\mathrm{~m}, 2 \mathrm{H}), 6.86(\mathrm{t}, J=7.9 \mathrm{~Hz}, 1 \mathrm{H}), 6.76(\mathrm{~s}, 1 \mathrm{H}), 5.54-5.26(\mathrm{~m}, 8 \mathrm{H}), 5.14(\mathrm{~s}, 2 \mathrm{H}), 4.81(\mathrm{dd}, J=7.3$, $6.4 \mathrm{~Hz}, 2 \mathrm{H}), 4.75(\mathrm{~s}, 2 \mathrm{H}), 4.65(\mathrm{dd}, J=11.2,5.6 \mathrm{~Hz}, 1 \mathrm{H}), 4.46(\mathrm{dd}, J=11.2,6.0 \mathrm{~Hz}, 1 \mathrm{H}), 4.42(\mathrm{~d}, J=7.5 \mathrm{~Hz}, 1 \mathrm{H}), 3.57(b r \mathrm{~s}$, $2 \mathrm{H}), 3.31-3.13(\mathrm{~m}, 2 \mathrm{H}), 2.73-2.52(\mathrm{~m}, 4 \mathrm{H}), 2.05(\mathrm{~s}, 3 \mathrm{H}), 1.60-1.56(\mathrm{~m}, 2 \mathrm{H}), 1.55(\mathrm{~d}, J=6.2 \mathrm{~Hz}, 3 \mathrm{H}), 1.46-1.36(\mathrm{~m}, 2 \mathrm{H})$. ${ }^{13} \mathrm{C}$ NMR $\left(125 \mathrm{MHz}, \mathrm{CDCl}_{3}\right) \delta 172.3,171.7,168.4,164.9,151.4,150.9,150.1,150.0,135.9,135.8,135.5,135.4,135.1,134.5$, 132.2, 132.2, 132.1, 129.3, 129.1, 129.0, 128.9, 128.7, 128.5, 128.4, 128.3, 125.2, 125.1, 124.2, 123.2, 121.4, 118.6, 88.4, 79.6, 79.2, 76.4 76.0, 75.8, 75.5, 75.3, 66.6, 64.2, 52.2, 44.8, 39.5, 33.6, 26.3, 24.5, 22.0, 20.6, 15.4. HR-MS (ESI-TOF) $\mathrm{m} / z$ for $\left[\mathrm{C}_{62} \mathrm{H}_{61} \mathrm{~N}_{4} \mathrm{O}_{13}\right]^{+}\left([\mathrm{M}+\mathrm{H}]^{+}\right)$: calculated 1069.4230, measured 1069.4256.

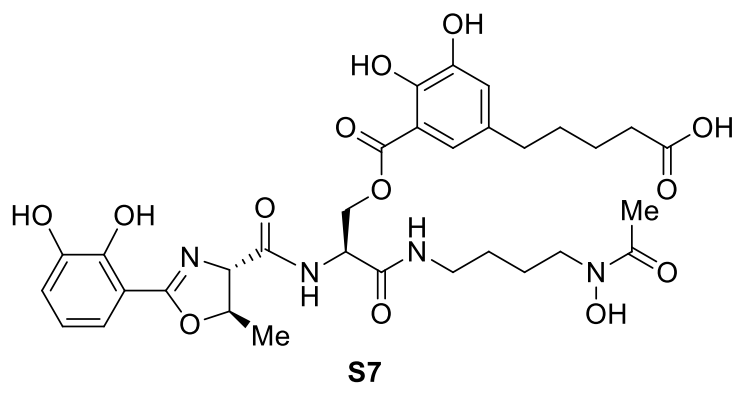

\section{5-(3-(((S)-2-((4S,5R)-2-(2,3-Dihydroxyphenyl)-5-methyl-4,5-dihydrooxazole-4-carboxamido)-3-((4-(N-} hydroxyacetamido)butyl)amino)-3-oxopropoxy)carbonyl)-4,5-dihydroxyphenyl)pentanoic acid (S7)

To a solution of $\mathbf{1 6 b}(65.7 \mathrm{mg}, 0.0615 \mathrm{mmol})$ in methanol $(2 \mathrm{~mL})$ and ethyl acetate $(1 \mathrm{~mL})$ was added a palladium on activated carbon $(10 \% \mathrm{Pd}, 65.4 \mathrm{mg}, 0.0615 \mathrm{mmol})$ at room temperature. This reaction mixture was then charged with hydrogen gas in a balloon. After stirred for $3 \mathrm{hr}$, the reaction mixture was filtered through a pad of celite to remove the catalyst and concentrated under reduced pressure to afford the desired product $\mathbf{S} 7$ (37.6 $\mathrm{mg}, 0.0547 \mathrm{mmol} 89 \%$ yield). This reaction progress was monitored by analytical HPLC (Acclaim $5 \mu \mathrm{m} \mathrm{C18} 120 \AA \AA$ column, DW/ACN = 95/5 to 20/80 over $15 \mathrm{~min}, 0.1 \%$ trifluoroacetic acid was added as an ion pairing agent, flow rate $=1.0 \mathrm{~min} / \mathrm{mL}, 1=254 \mathrm{~nm}), t_{\mathrm{R}}=10.4 \mathrm{~min}(\mathbf{S} 7), t_{\mathrm{R}}=17.6 \mathrm{~min}(\mathbf{1 6 b}) .{ }^{1} \mathrm{H} \mathrm{NMR}$ $\left(500 \mathrm{MHz}, \mathrm{CD}_{3} \mathrm{OD}\right) \delta 7.13(\mathrm{dd}, J=8.0,1.4 \mathrm{~Hz}, 1 \mathrm{H}), 7.06(\mathrm{~d}, J=1.7 \mathrm{~Hz}, 1 \mathrm{H}), 6.94(\mathrm{dd}, J=7.9,1.4 \mathrm{~Hz}, 1 \mathrm{H}), 6.82(\mathrm{~d}, J=1.9$ $\mathrm{Hz}, 1 \mathrm{H}), 6.73(\mathrm{t}, J=7.9 \mathrm{~Hz}, 1 \mathrm{H}), 4.94-4.83(\mathrm{~m}, 2 \mathrm{H}), 4.73(\mathrm{dd}, J=11.3,4.5 \mathrm{~Hz}, 1 \mathrm{H}), 4.59-4.56(\mathrm{~m}, 1 \mathrm{H}), 4.57-4.53(\mathrm{~m}$, $1 \mathrm{H}), 3.59$ (t, $J=6.7 \mathrm{~Hz}, 2 \mathrm{H}), 3.27$ (td, $J=6.8,2.7 \mathrm{~Hz}, 2 \mathrm{H}), 2.41(\mathrm{t}, J=6.9 \mathrm{~Hz}, 2 \mathrm{H}), 2.26(\mathrm{t}, J=6.9 \mathrm{~Hz}, 2 \mathrm{H}), 2.08(\mathrm{~s}, 3 \mathrm{H}), 1.69$ $-1.60(\mathrm{~m}, 2 \mathrm{H}), 1.59-1.52(\mathrm{~m}, 6 \mathrm{H}), 1.53(\mathrm{~d}, J=6.3 \mathrm{~Hz}, 3 \mathrm{H}) .{ }^{13} \mathrm{C} \mathrm{NMR}\left(125 \mathrm{MHz}, \mathrm{CD}_{3} \mathrm{OD}\right) \delta 178.4,173.6,173.0,171.2$, $170.4,168.5,149.5,149.4,146.8,146.7,134.5,133.8,133.1,133.0,130.0,129.9,122.3,120.4,120.4,119.9,119.9,112.9$, 
111.6, 80.4, 75.5, 65.2, 53.7, 40.2, 35.7, 35.4, 31.9, 27.3, 25.8, 25.0, 21.3, 20.2. HR-MS (ESI-TOF) $m / z$ for $\left[\mathrm{C}_{32} \mathrm{H}_{40} \mathrm{~N}_{4} \mathrm{NaO}_{13}\right]^{+}$ $\left([\mathrm{M}+\mathrm{Na}]^{+}\right)$: calculated 711.2484, measured 711.2484.

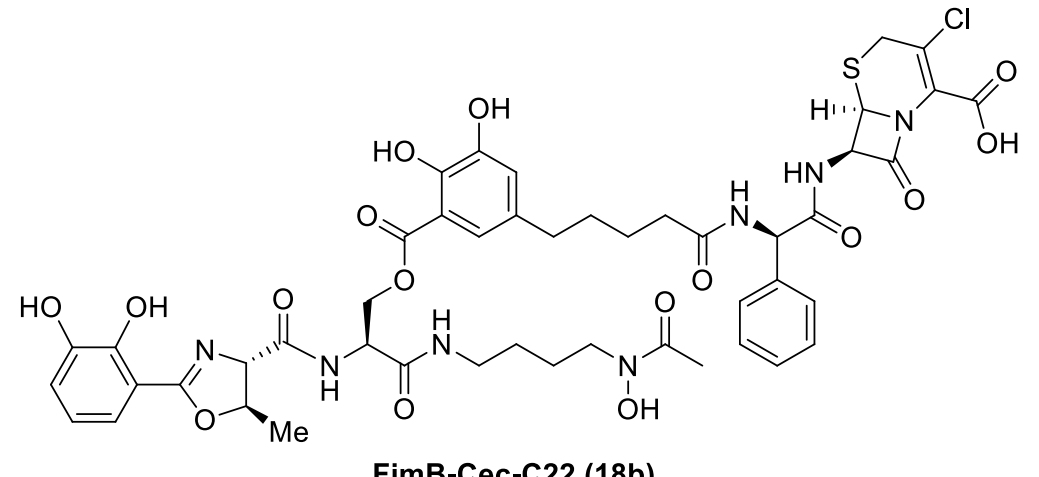

(6R)-3-Chloro-7-((R)-2-(5-(3-(((S)-2-((4S,5R)-2-(2,3-dihydroxyphenyl)-5-methyl-4,5-dihydrooxazole-4-carboxamido)3-((4-(N-hydroxyacetamido)butyl)amino)-3-oxopropoxy)carbonyl)-4,5-

dihydroxyphenyl)pentanamido)-2-phenylacetamido)-8-oxo-5-thia-1-azabicyclo[4.2.0]oct-2-ene-2-carboxylic acid

(FimB-Cec-C22, 18b)

To a solution of compound $\mathbf{S} 7(17.3 \mathrm{mg}, 0.0251 \mathrm{mmol})$ in DMF $(100 \mu \mathrm{L})$ was added EDC (7.22 $\mathrm{mg}, 0.0377 \mathrm{mmol})$ and NHS (4.34 mg, $0.0377 \mathrm{mmol}$ ) at room temperature. This reaction progress was monitored by analytical HPLC (Acclaim $5 \mu \mathrm{m} \mathrm{C18}$ $120 \AA$ column, $\mathrm{DW} / \mathrm{ACN}=95 / 5$ to $20 / 80$ for $15 \mathrm{~min}, 0.1 \%$ trifluoroacetic acid was added as an ion pairing agent, flow rate $=$ $1.0 \mathrm{~min} / \mathrm{mL}, 1=254 \mathrm{~nm}), t_{\mathrm{R}}=10.4 \mathrm{~min}(\mathbf{S} 7), t_{\mathrm{R}}=11.4 \mathrm{~min}(\mathrm{NHS}-\mathrm{ester}), t_{\mathrm{R}}=11.9 \mathrm{~min}(\mathbf{1 8 b})$. After $4.5 \mathrm{~h}$ stirring, approximately $75 \%$ of compound $\mathbf{S} 7$ to the corresponding NHS-ester was converted, but further progression was not observed. To the resulting solution of NHS-ester in DMF was added a solution of cefaclor $(13.9 \mathrm{mg}, 0.0377 \mathrm{mmol})$ and diisopropylethyl amine $(17.5 \mu \mathrm{L}$, $0.100 \mathrm{mmol})$ in dimethyl sulfoxide $(300 \mu \mathrm{L})$ at room temperature. After $2 \mathrm{~h}$ stirring, approximately $86 \%$ conversion of the NHS ester intermediate to the corresponding cefaclor conjugate $\mathbf{1 8 b}$ was observed. The crude reaction mixture was purified by semipreparative HPLC (Luna $5 \mu \mathrm{m} \mathrm{C18(2)} 100 \AA$ column, DW/ACN = 80/20 to 65/35 over $50 \mathrm{~min}, 10 \mathrm{mM}$ ammonium bicarbonate was added as an ion pairing agent for $\mathrm{DW}$ and $\mathrm{pH}=7.2$, flow rate $=5.0 \mathrm{~mL} / \mathrm{min}, 1=254 \mathrm{~nm}), t_{\mathrm{R}}=24.5 \mathrm{~min}(\mathbf{1 8 b})$. After lyophilization, the desired product $\mathbf{1 8 b}$ was afforded (5.6 mg, $5.4 \mu \mathrm{mol}$, two-step $27 \%$ yield) as a white solid. ${ }^{1} \mathrm{H}$ NMR (500 $\left.\mathrm{MHz}, \mathrm{CD}_{3} \mathrm{OD}\right) \delta 7.43(\mathrm{~d}, J=6.8 \mathrm{~Hz}, 2 \mathrm{H}), 7.37-7.27(\mathrm{~m}, 3 \mathrm{H}), 7.12(\mathrm{dd}, J=8.0,1.5 \mathrm{~Hz}, 1 \mathrm{H}), 7.05(\mathrm{~d}, J=1.8 \mathrm{~Hz}, 1 \mathrm{H}), 6.94$ $(\mathrm{dd}, J=7.9,1.5 \mathrm{~Hz}, 1 \mathrm{H}), 6.79(\mathrm{~d}, J=1.9 \mathrm{~Hz}, 1 \mathrm{H}), 6.72(\mathrm{t}, J=7.9 \mathrm{~Hz}, 1 \mathrm{H}), 5.68(\mathrm{~d}, J=4.8 \mathrm{~Hz}, 1 \mathrm{H}), 5.56(\mathrm{~s}, 1 \mathrm{H}), 5.00(\mathrm{~d}, J=$ $4.8 \mathrm{~Hz}, 1 \mathrm{H}), 4.94-4.83(\mathrm{~m}, 2 \mathrm{H}), 4.77(\mathrm{dd}, J=11.3,4.6 \mathrm{~Hz}, 1 \mathrm{H}), 4.54$ (d, $J=7.1 \mathrm{~Hz}, 1 \mathrm{H}), 4.51$ (dd, $J=11.4,7.0 \mathrm{~Hz}, 1 \mathrm{H}), 3.72$ $(\mathrm{d}, J=17.6 \mathrm{~Hz}, 1 \mathrm{H}), 3.58$ (t, $J=6.7 \mathrm{~Hz}, 2 \mathrm{H}), 3.26(\mathrm{t}, J=6.2 \mathrm{~Hz}, 2 \mathrm{H}), 2.39$ (t, $J=7.0 \mathrm{~Hz}, 2 \mathrm{H}), 2.28$ (ddd, $J=36.9,14.3,7.3$ $\mathrm{Hz}, 2 \mathrm{H}), 2.07(\mathrm{~s}, 3 \mathrm{H}), 1.67-1.60(\mathrm{~m}, 2 \mathrm{H}), 1.59-1.51(\mathrm{~m}, 6 \mathrm{H}), 1.50(\mathrm{~d}, J=6.3 \mathrm{~Hz}, 3 \mathrm{H}) .{ }^{13} \mathrm{C}$ NMR $\left(125 \mathrm{MHz}\right.$, DMSO-d $\left.\mathrm{d}^{6}\right) \delta$ $171.9,170.9,170.5,170.2,169.9,168.9,167.9,166.0,148.3,147.6,145.8,138.2,132.6,128.2,127.5,127.1,127.1,121.2$, 119.4, 118.8, 118.6, 117.9, 112.3, 110.2, 78.8, 73.4, 64.4, 59.1, 58.1, 57.1, 55.6, 52.9, 51.5, 46.4, 38.5, 34.7, 34.1, 30.5, 29.4, 26.0, 24.8, 23.7, 20.5, 20.3. HR-MS (ESI-TOF) $\mathrm{m} / z$ for $\left[\mathrm{C}_{47} \mathrm{H}_{52} \mathrm{ClN}_{7} \mathrm{NaO}_{16} \mathrm{~S}\right]^{+}\left([\mathrm{M}+\mathrm{Na}]^{+}\right)$: calculated 1060.2772, measured 1060.2745 . 


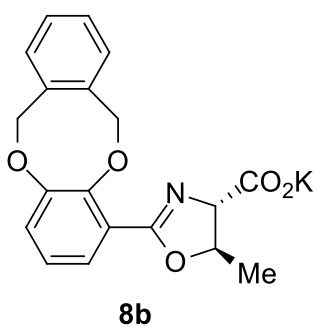

Potassium (4S,5R)-2-(6,11-dihydrodibenzo[b,f][1,4]dioxocin-1-yl)-5-methyl-4,5-dihydrooxazole-4-carboxylate (8b)

Compound $\mathbf{8 b}$ was prepared as previously reported (Bull. Kor. Chem. Soc. 2015, 36, 439). ${ }^{1} \mathrm{H}$ NMR (500 MHz, CD $\left.{ }_{3} \mathrm{OD}\right) \delta 7.38$ (dd, $J=7.7,1.7 \mathrm{~Hz}, 1 \mathrm{H}), 7.30-7.21(\mathrm{~m}, 4 \mathrm{H}), 7.07$ (dd, $J=8.0,1.6 \mathrm{~Hz}, 1 \mathrm{H}), 6.94(\mathrm{t}, J=7.9 \mathrm{~Hz}, 1 \mathrm{H}), 5.53-5.36(\mathrm{~m}, 4 \mathrm{H}), 4.89$ $-4.82(\mathrm{~m}, 1 \mathrm{H}), 4.26(\mathrm{~d}, J=6.9 \mathrm{~Hz}, 1 \mathrm{H}), 1.52(\mathrm{~d}, J=6.3 \mathrm{~Hz}, 3 \mathrm{H})$.

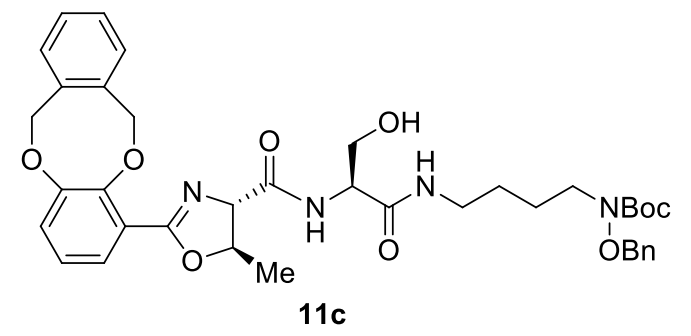

tert-butyl Benzyloxy(4-((S)-2-((4S,5R)-2-(6,11-dihydrodibenzo[b,f][1,4]dioxocin-1-yl)-5-methyl-4,5-dihydrooxazole $\quad-4-$ carboxamido)-3-hydroxypropanamido)butyl)carbamate (11c)

To a solution of amine $10 \mathbf{a}(2.31 \mathrm{~g}, 4.66 \mathrm{mmol})$ and potassium carboxylate $\mathbf{8 b}(2.64 \mathrm{~g}, 6.99 \mathrm{mmol})$ in DMF (12 $\mathrm{mL})$ was added $\mathrm{EDC}(1.79 \mathrm{~g}, 9.32 \mathrm{mmol})$ and $\mathrm{HOBt}(1.43 \mathrm{~g}, 9.32 \mathrm{mmol})$ at $0{ }^{\circ} \mathrm{C}$. After stirred for $6 \mathrm{~h}$, the reaction mixture was concentrated under reduced pressure. The resulting residue was then diluted with ethyl acetate, washed with brine, and dried over anhydrous magnesium sulfate. After concentration under reduced pressure, the crude residue was purified by flash column chromatography ( $\mathrm{SiO}_{2}, 1 \%$ methanol in ethyl acetate) to afford the crude, silyl protected intermediate ( $2.89 \mathrm{~g}, 3.54 \mathrm{mmol}$, crude $76 \%$ yield) which was directly used in the following deprotection step. This intermediate was dissolved in tetrahydrofuran (5 $\mathrm{mL})$ and treated with TBAF $(1.0 \mathrm{M}$ in tetrahydrofuran, $5.31 \mathrm{~mL}, 5.31 \mathrm{mmol})$ at $0{ }^{\circ} \mathrm{C}$. The reaction mixture was stirred for $1 \mathrm{~h}$ at room temperature, and then the solvent was removed by vacuo. The crude residue was then purified by flash column chromatography $\left(\mathrm{SiO}_{2}, 3 \%\right.$ methanol in ethyl acetate) to afford the desired product 11c (795 mg, $1.13 \mathrm{mmol}$, two-step 32\% yield) as a white solid. ${ }^{1} \mathrm{H} \mathrm{NMR}\left(500 \mathrm{MHz}, \mathrm{CDCl}_{3}\right) \delta 7.86(\mathrm{~d}, J=7.6 \mathrm{~Hz}, 1 \mathrm{H}), 7.46-7.12(\mathrm{~m}, 11 \mathrm{H}), 6.95(\mathrm{t}, J=7.9 \mathrm{~Hz}, 2 \mathrm{H})$, $5.48(\mathrm{ddd}, J=75.6,73.9,13.2 \mathrm{~Hz}, 4 \mathrm{H}), 4.85(\mathrm{dd}, J=13.5,6.4 \mathrm{~Hz}, 1 \mathrm{H}), 4.80(\mathrm{~s}, 2 \mathrm{H}), 4.44(\mathrm{~d}, J=7.5 \mathrm{~Hz}, 1 \mathrm{H}), 4.43-4.40$ (m, 1H), $4.08(\mathrm{dd}, J=11.4,3.3 \mathrm{~Hz}, 1 \mathrm{H}), 3.63(\mathrm{dd}, J=11.5,4.7 \mathrm{~Hz}, 1 \mathrm{H}), 3.41(\mathrm{t}, J=7.0 \mathrm{~Hz}, 2 \mathrm{H}), 3.25(\mathrm{ddd}, J=13.0,9.8,6.9 \mathrm{~Hz}$, $2 \mathrm{H}), 1.65-1.58(\mathrm{~m}, 2 \mathrm{H}), 1.57(\mathrm{~d}, J=6.3 \mathrm{~Hz}, 3 \mathrm{H}), 1.54-1.50(\mathrm{~m}, 2 \mathrm{H}), 1.49(\mathrm{~s}, 9 \mathrm{H}) .{ }^{13} \mathrm{C} \mathrm{NMR}\left(125 \mathrm{MHz}, \mathrm{CDCl}_{3}\right) \delta 172.5$, 170.6, 164.2, 156.7, 151.3, 150.3, 135.6, 135.6, 129.5, 129.3, 128.7, 128.7, 128.7, 128.6, 125.8, 125.3, 123.2, 120.7, 81.6, 79.1, 
75.7, 75.6, 75.5, 62.6, 54.0, 49.1, 39.2, 28.4, 26.6, 24.4, 21.9. HR-MS (ESI-TOF) $m / z$ for $\left[\mathrm{C}_{38} \mathrm{H}_{47} \mathrm{~N}_{4} \mathrm{O}_{9}\right]^{+}\left([\mathrm{M}+\mathrm{H}]^{+}\right)$: calculated 703.3338 , measured 703.3342 .

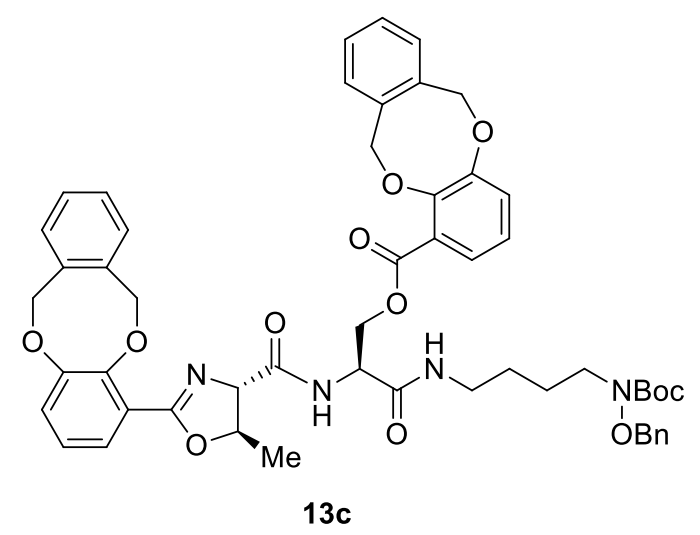

(S)-3-((4-((Benzyloxy)(tert-butoxycarbonyl)amino)butyl)amino)-2-((4S,5R)-2-(6,11-dihydrodibenzo[b,f][1,4]dioxoc in-1-yl)-5-methyl-4,5-dihydrooxazole-4-carboxamido)-3-oxopropyl 6,11-dihydrodibenzo[b,f][1,4]dioxocine-1-carbo xyl ate (13c)

To a solution of compound 11c $(690 \mathrm{mg}, 0.982 \mathrm{mmol})$ and triethylamine $(298 \mu \mathrm{L}, 2.95 \mathrm{mmol})$ in dichloromethane $(4 \mathrm{~mL})$ was added a solution of acid chloride $\mathbf{1 2 b}(485 \mathrm{mg}, 1.77 \mathrm{mmol})$ in dichloromethane $(1 \mathrm{~mL})$ dropwise at $0{ }^{\circ} \mathrm{C}$, followed by treatment of 4-dimethylaminopyridine (DMAP, $60.0 \mathrm{mg}, 0.491 \mathrm{mmol}$ ). After stirred at room temperature for $1 \mathrm{~h}$, the solvent was removed under reduced pressure. The crude residue was purified by flash column chromatography $\left(\mathrm{SiO}_{2}, 3 \%\right.$ methanol in ethyl acetate) to afford the desired product 13c $\left(896 \mathrm{mg}, 0.952 \mathrm{mmol}, 97 \%\right.$ yield) as a white solid. ${ }^{1} \mathrm{H}$ NMR $\left(500 \mathrm{MHz}, \mathrm{CDCl}_{3}\right) \delta 7.66(\mathrm{~d}, J$ $=7.4 \mathrm{~Hz}, 1 \mathrm{H}), 7.40-7.07(\mathrm{~m}, 17 \mathrm{H}), 6.87(\mathrm{t}, J=7.9 \mathrm{~Hz}, 1 \mathrm{H}), 6.79(\mathrm{t}, J=7.9 \mathrm{~Hz}, 1 \mathrm{H}), 6.58(\mathrm{t}, J=5.6 \mathrm{~Hz}, 1 \mathrm{H}), 5.57-5.23(\mathrm{~m}$, $8 \mathrm{H}), 4.83-4.78(\mathrm{~m}, 2 \mathrm{H}), 4.78(\mathrm{~s}, 2 \mathrm{H}), 4.66(\mathrm{dd}, J=11.2,5.5 \mathrm{~Hz}, 1 \mathrm{H}), 4.46(\mathrm{dd}, J=11.2,6.1 \mathrm{~Hz}, 1 \mathrm{H}), 4.41(\mathrm{~d}, J=7.5 \mathrm{~Hz}, 1 \mathrm{H})$, 3.34 (t, $J=7.0 \mathrm{~Hz}, 2 \mathrm{H}), 3.26-3.13(\mathrm{~m}, 2 \mathrm{H}), 1.56(\mathrm{~d}, J=6.2 \mathrm{~Hz}, 3 \mathrm{H}), 1.53-1.47(\mathrm{~m}, 2 \mathrm{H}), 1.48(\mathrm{~s}, 9 \mathrm{H}), 1.42-1.35(\mathrm{~m}, 2 \mathrm{H})$. ${ }^{13} \mathrm{C}$ NMR $\left(125 \mathrm{MHz}, \mathrm{CDCl}_{3}\right) \delta 172.3,168.3,165.4,164.3,156.7,151.5,151.4,150.2,150.0,135.8,135.6,135.5,135.5,135.5$, $129.5,129.0,128.9,128.8,128.8,128.7,128.7,128.6,126.2,125.7,125.2,125.1,124.2,123.3,123.2,121.4,81.5,79.2,76.0$, 75.9, 75.7, 75.3, 64.0, 52.2, 49.0, 39.6, 28.4, 26.5, 24.5, 22.0. HR-MS (ESI-TOF) $m / z$ for $\left[\mathrm{C}_{53} \mathrm{H}_{57} \mathrm{~N}_{4} \mathrm{O}_{12}\right]^{+}\left([\mathrm{M}+\mathrm{H}]^{+}\right)$: calculated 941.3967, measured 941.3973 .<smiles>O=C(O)CCC(=O)NCCC(=O)Oc1ccccc1</smiles>

\section{4-((3-(benzyloxy)-3-oxopropyl)amino)-4-oxobutanoic acid (15)}

Compound 15 was prepared by same method as previously reported (J. Med. Chem. 2002, 45, 3, 598). ${ }^{1} \mathrm{H}$ NMR (500 MHz, $\left.\mathrm{CDCl}_{3}\right) \delta 7.41-7.32(\mathrm{~m}, 5 \mathrm{H}), 6.28(\mathrm{~s}, 1 \mathrm{H}), 5.15(\mathrm{~s}, 2 \mathrm{H}), 3.55(\mathrm{dd}, J=11.9,6.1 \mathrm{~Hz}, 2 \mathrm{H}), 2.67(\mathrm{dd}, J=7.4,5.5 \mathrm{~Hz}, 2 \mathrm{H}), 2.62-$ 


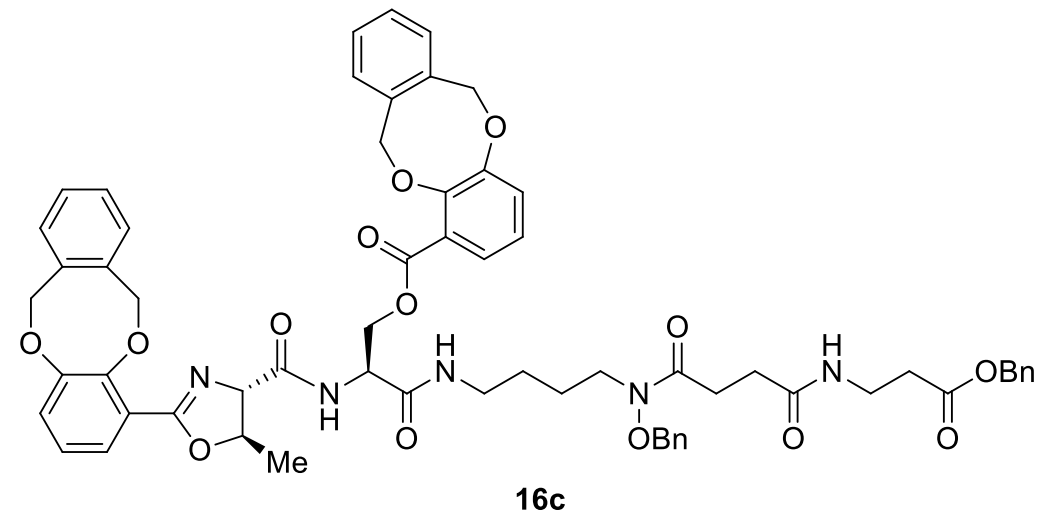

(S)-Benzyl 10-(benzyloxy)-1-((4S,5R)-2-(6,11-dihydrodibenzo[b,f][1,4]dioxocin-1-yl)-5-methyl-4,5-dihydrooxazol-4-yl)3-(((6,11-dihydrodibenzo[b,f][1,4]dioxocine-1-carbonyl)oxy)methyl)-1,4,11,14-tetraoxo-2,5,10,15-tetraazaoctadecan18-oate (16c)

To a solution of $13 \mathbf{c}(800 \mathrm{mg}, 0.850 \mathrm{mmol})$ in dichloromethane $(2 \mathrm{~mL})$ was added trifluoroacetic acid (TFA, $400 \mu \mathrm{L})$ at room temperature. The reaction mixture was stirred for $8 \mathrm{~h}$, and then the solvent was removed under reduced pressure. The resulting TFA salt residue was basified with saturated sodium bicarbonate solution and extracted three times with dichloromethane. The combined organic layers were then washed with brine, dried over anhydrous magnesium sulfate, and concentrated under reduced pressure. The resulting crude intermediate was used for the next step without further purification. To a mixture of this intermediate and carboxylic acid 15 (356 mg, 1.28mmol) in dimethylformamide (4 mL) was added EDC (326 mg, $1.70 \mathrm{mmol})$, HOBt $(260 \mathrm{~g}, 1.70 \mathrm{mmol})$, and diisopropylethyl amine $(740 \mu \mathrm{L}, 4.25 \mathrm{mmol})$ at room temperature, after which time the temperature was elevated with an oil bath to $100^{\circ} \mathrm{C}$. After stirred for $1 \mathrm{~h}$, the reaction mixture was concentrated under reduced pressure. The resulting residue was then diluted with ethyl acetate, washed with brine, and dried over anhydrous magnesium sulfate. After concentration under reduced pressure, the crude residue was purified by flash column chromatography $\left(\mathrm{SiO}_{2}, 4 \%\right.$ methanol in dichloromethane) to afford the desired product $16 \mathrm{c}$ (450 mg, $0.408 \mathrm{mmol}$, two-step 48\% yield) as a white solid. ${ }^{1} \mathrm{H} \mathrm{NMR}\left(500 \mathrm{MHz}, \mathrm{CDCl}_{3}\right) \delta 7.69(\mathrm{~d}, J=7.6 \mathrm{~Hz}, 1 \mathrm{H}), 7.42-6.92(\mathrm{~m}, 22 \mathrm{H}), 6.97(\mathrm{~d}, J=5.2 \mathrm{~Hz}, 1 \mathrm{H}), 6.84(\mathrm{t}, J=7.9 \mathrm{~Hz}, 1 \mathrm{H})$, $6.73(\mathrm{t}, J=7.9 \mathrm{~Hz}, 1 \mathrm{H}), 6.44(\mathrm{~d}, J=5.7 \mathrm{~Hz}, 1 \mathrm{H}), 5.56-5.23(\mathrm{~m}, 8 \mathrm{H}), 5.11(\mathrm{~s}, 2 \mathrm{H}), 4.88-4.80(\mathrm{~m}, 2 \mathrm{H}), 4.80(\mathrm{~s}, 2 \mathrm{H}), 4.64(\mathrm{dd}$, $J=11.2,5.4 \mathrm{~Hz}, 1 \mathrm{H}), 4.52(\mathrm{dd}, J=11.2,5.6 \mathrm{~Hz}, 1 \mathrm{H}), 4.41(\mathrm{~d}, J=7.4 \mathrm{~Hz}, 1 \mathrm{H}), 3.57(b r \mathrm{~s}, 2 \mathrm{H}), 3.49(\mathrm{dd}, J=12.3,6.1 \mathrm{~Hz}, 2 \mathrm{H})$, $3.22(\mathrm{dd}, J=13.1,6.5 \mathrm{~Hz}, 2 \mathrm{H}), 2.72(b r \mathrm{~s}, 2 \mathrm{H}), 2.55(\mathrm{t}, J=6.1 \mathrm{~Hz}, 2 \mathrm{H}), 2.41(\mathrm{t}, J=6.4 \mathrm{~Hz}, 2 \mathrm{H}), 1.61-1.55(\mathrm{~m}, 2 \mathrm{H}), 1.54(\mathrm{~d}$, $J=6.2 \mathrm{~Hz}, 3 \mathrm{H}), 1.41$ (dt, $J=13.3,6.5 \mathrm{~Hz}, 2 \mathrm{H}) .{ }^{13} \mathrm{C} \mathrm{NMR}\left(125 \mathrm{MHz}, \mathrm{CDCl}_{3}\right) \delta 172.4,172.4,172.2,168.4,165.4,164.3,151.4$, $151.4,150.2,149.9,135.8,135.8,135.6,135.5,135.5,129.3,129.1,129.1,129.0,129.0,128.9,128.8,128.8,128.7,128.6$, 128.4, 128.3, 126.1, 125.7, 125.2, 125.1, 124.4, 123.3, 121.5, 79.2, 76.5, 76.0, 75.9, 75.8, 75.2, 75.2, 66.6, 64.2, 59.2, 52.2, 39.3, 35.1, 34.2, 30.6, 27.9, 26.0, 24.3, 22.0. HR-MS (ESI-TOF) $\mathrm{m} / z$ for $\left[\mathrm{C}_{62} \mathrm{H}_{64} \mathrm{~N}_{5} \mathrm{O}_{14}\right]^{+}\left([\mathrm{M}+\mathrm{H}]^{+}\right)$: calculated 1102.4444, measured 1102.4447. 


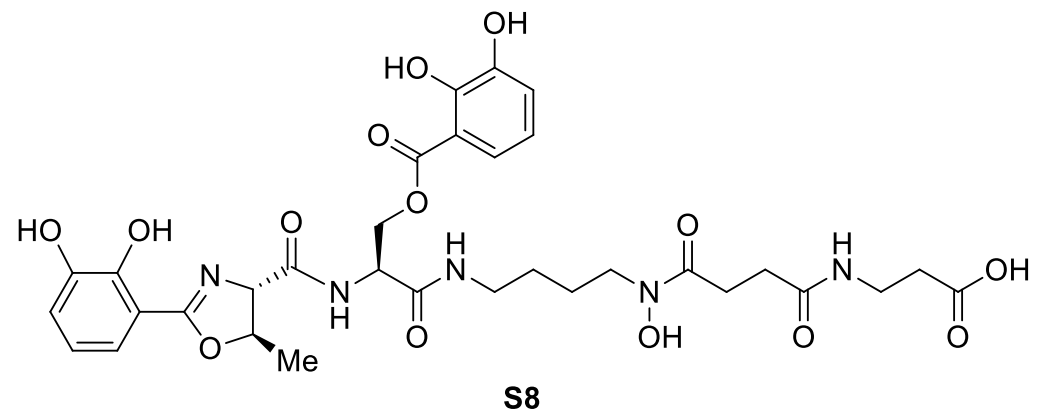

(S)-1-(2,3-dihydroxyphenyl)-4-((4S,5R)-2-(2,3-dihydroxyphenyl)-5-methyl-4,5-dihydrooxazole-4-carboxamido)-11hydroxy-1,5,12,15-tetraoxo-2-oxa-6,11,16-triazanonadecan-19-oic acid (S8)

To a solution of compound 16c $(100 \mathrm{mg}, 0.0907 \mathrm{mmol})$ in methanol $(2 \mathrm{~mL})$ and ethyl acetate $(1 \mathrm{~mL}) \mathrm{was}$ added a palladium on activated carbon $(10 \% \mathrm{Pd} / \mathrm{C}, 96.6 \mathrm{mg}, 0.0907 \mathrm{mmol})$ at room temperature. This reaction mixture was charged with hydrogen gas in a balloon. After stirred for $3 \mathrm{~h}$, the reaction mixture was filtered through a pad of celite to remove the catalyst, and the filtrate was concentrated under reduced pressure to afford the desired product $\mathbf{S 8}$ (57.9 mg, $0.0808 \mathrm{mmol} 89 \%$ yield). This reaction progress was monitored by analytical HPLC (Acclaim $5 \mu \mathrm{m} \mathrm{C18} 120 \AA$ column, DW/ACN = 95/5 to 20/80 over 15 min, $0.1 \%$ trifluoroacetic acid was added as an ion pairing agent, flow rate $=1.0 \mathrm{~min} / \mathrm{mL}, 1=254 \mathrm{~nm}), t_{\mathrm{R}}=9.9 \mathrm{~min}(\mathbf{S 8}), t_{\mathrm{R}}=$ $16.4 \mathrm{~min}(\mathbf{1 6 c}) .{ }^{1} \mathrm{H}$ NMR $\left(500 \mathrm{MHz}, \mathrm{CD}_{3} \mathrm{OD}\right) \delta 7.30-7.20(\mathrm{~m}, 2 \mathrm{H}), 7.06(\mathrm{t}, J=9.7 \mathrm{~Hz}, 1 \mathrm{H}), 6.96(\mathrm{dd}, J=7.8,1.3 \mathrm{~Hz}, 1 \mathrm{H})$, $6.82(\mathrm{t}, J=8.0 \mathrm{~Hz}, 1 \mathrm{H}), 6.65(\mathrm{t}, J=8.0 \mathrm{~Hz}, 1 \mathrm{H}), 5.17-5.11(\mathrm{~m}, 1 \mathrm{H}), 4.85-4.80(\mathrm{~m}, 1 \mathrm{H}), 4.70(\mathrm{dd}, J=11.2,4.6 \mathrm{~Hz}, 2 \mathrm{H}), 4.70$ $(\mathrm{d}, J=6.5 \mathrm{~Hz}, 1 \mathrm{H}), 4.59(\mathrm{dd}, J=11.2,6.4 \mathrm{~Hz}, 1 \mathrm{H}), 3.58(\mathrm{t}, J=6.6 \mathrm{~Hz}, 2 \mathrm{H}), 3.41(\mathrm{t}, J=6.8 \mathrm{~Hz}, 2 \mathrm{H}), 3.30-3.23(\mathrm{~m}, 2 \mathrm{H}), 2.74$ $(\mathrm{t}, J=7.0 \mathrm{~Hz}, 2 \mathrm{H}), 2.50(\mathrm{t}, J=6.8 \mathrm{~Hz}, 2 \mathrm{H}), 2.44(\mathrm{t}, J=7.0 \mathrm{~Hz}, 2 \mathrm{H}), 1.68-1.61(\mathrm{~m}, 2 \mathrm{H}), 1.62(\mathrm{~d}, J=6.3 \mathrm{~Hz}, 3 \mathrm{H}), 1.58-1.46$ (m, 2H). ${ }^{13} \mathrm{C}$ NMR (125 MHz, CD $\left.3 \mathrm{OD}\right) \delta 175.3,175.1,174.6,171.2,170.3,151.2,147.1,147.0,122.0,121.6,121.4,120.6$, $120.4,120.2,113.4,79.5,79.3,79.0,65.3,53.8,40.3,36.4,36.3,34.7,34.6,31.3,28.7,27.2$, 25.0, 20.9, 17.4. HR-MS (ESITOF) $m / z$ for $\left[\mathrm{C}_{32} \mathrm{H}_{40} \mathrm{~N}_{5} \mathrm{O}_{14}\right]^{+}\left([\mathrm{M}+\mathrm{H}]^{+}\right)$: calculated 718.2566, measured 718.2570.

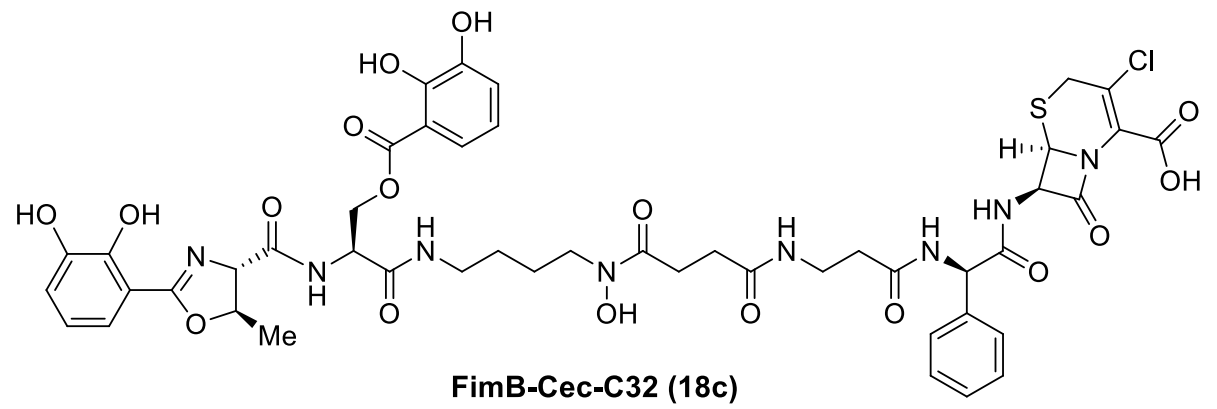

(6R)-3-Chloro-7-((4S,21R)-1-(2,3-dihydroxyphenyl)-4-((4S,5R)-2-(2,3-dihydroxyphenyl)-5-methyl-4,5-dihydrooxazole4-carboxamido)-11-hydroxy-1,5,12,15,19-pentaoxo-21-phenyl-2-oxa-6,11,16,20-tetraazadocosanamido)-8-oxo-5-thia-1azabicyclo[4.2.0]oct-2-ene-2-carboxylic acid (FimB-Cec-C32, 18c)

To a solution of $\mathbf{S 8}(21.6 \mathrm{mg}, 0.0301 \mathrm{mmol})$ in DMF $(300 \mu \mathrm{L})$ was added EDC (8.65 mg, $0.0452 \mathrm{mmol})$ and NHS (5.20 mg, 
$0.0452 \mathrm{mmol}$ ) at room temperature. This reaction progress was monitored by analytical HPLC (Acclaim $5 \mu \mathrm{m}$ C18 $120 \AA$ column, $\mathrm{DW} / \mathrm{ACN}=95 / 5$ to $20 / 80$ for $15 \mathrm{~min}, 0.1 \%$ trifluoroacetic acid was added as an ion pairing agent, flow rate $=1.0$ $\min / \mathrm{mL}, 1=254 \mathrm{~nm}), t_{\mathrm{R}}=9.9 \mathrm{~min}(\mathbf{S 8}), t_{\mathrm{R}}=10.6 \mathrm{~min}(\mathrm{NHS}-\mathrm{ester}), t_{\mathrm{R}}=11.5 \mathrm{~min}(\mathbf{1 8 c})$. After $3 \mathrm{~h}$ stirring, approximately $82 \%$ of compound S8 to the corresponding NHS-ester was converted, but further progression was not observed. To the resulting solution of the NHS-ester in DMF was added a solution of cefaclor (16.6 mg, $0.0452 \mathrm{mmol}$ ) and diisopropylethyl amine (62.9 $\mu \mathrm{L}, 0.361 \mathrm{mmol})$ in dimethyl sulfoxide $(300 \mu \mathrm{L})$ at room temperature. After $2 \mathrm{~h}$ stirring, near $100 \%$ conversion of the NHS ester intermediate to the corresponding cefaclor conjugate $18 \mathbf{c}$ was observed. The crude reaction mixture was purified by semipreparative HPLC (Luna $5 \mu \mathrm{m} \mathrm{C18(2)} 100 \AA$ column, DW/ACN = 85/15 to 70/30 over 50 min, 10 mM ammonium bicarbonate was added as an ion pairing agent for $\mathrm{DW}$ and $\mathrm{pH}=7.2$, flow rate $=5.0 \mathrm{~mL} / \mathrm{min}, 1=254 \mathrm{~nm}), t_{\mathrm{R}}=36.3 \mathrm{~min}(\mathbf{1 8 c})$. After lyophilization, the desired product 18c was afforded (5.0 mg, $4.7 \mu \mathrm{mol}$, two-step $23 \%$ yield) as a white solid. ${ }^{1} \mathrm{H} \mathrm{NMR}(500$ $\left.\mathrm{MHz}, \mathrm{CD}_{3} \mathrm{OD}\right) \delta 7.44(\mathrm{~d}, J=6.8 \mathrm{~Hz}, 2 \mathrm{H}), 7.38-7.27(\mathrm{~m}, 3 \mathrm{H}), 7.21(\mathrm{~d}, J=8.1 \mathrm{~Hz}, 1 \mathrm{H}), 7.14(\mathrm{~d}, J=7.9 \mathrm{~Hz}, 1 \mathrm{H}), 6.94(\mathrm{dd}, J$ $=10.5,8.9 \mathrm{~Hz}, 2 \mathrm{H}), 6.74(\mathrm{t}, J=7.9 \mathrm{~Hz}, 1 \mathrm{H}), 6.58(\mathrm{t}, J=7.9 \mathrm{~Hz}, 1 \mathrm{H}), 5.67(\mathrm{~d}, J=4.2 \mathrm{~Hz}, 1 \mathrm{H}), 5.52(\mathrm{~s}, 1 \mathrm{H}), 5.02(\mathrm{~d}, J=3.7$ $\mathrm{Hz}, 1 \mathrm{H}), 4.94-4.79(\mathrm{~m}, 2 \mathrm{H}), 4.77-4.69(\mathrm{~m}, 1 \mathrm{H}), 4.56(\mathrm{~d}, J=6.3 \mathrm{~Hz}, 2 \mathrm{H}), 3.75(\mathrm{~d}, J=17.7 \mathrm{~Hz}, 1 \mathrm{H}), 3.59-3.51(\mathrm{~m}, 2 \mathrm{H})$, $3.28-3.17(\mathrm{~m}, 4 \mathrm{H}), 2.75(\mathrm{~s}, 2 \mathrm{H}), 2.47-2.39(\mathrm{~m}, 2 \mathrm{H}), 1.68-1.57(\mathrm{~m}, 2 \mathrm{H}), 1.53(\mathrm{~d}, J=14.1 \mathrm{~Hz}, 3 \mathrm{H}), 1.55-1.47(\mathrm{~m}, 4 \mathrm{H}) .{ }^{13} \mathrm{C}$ NMR (125 MHz, DMSO-d $\left.{ }^{6}\right) \delta 172.0,171.5,170.9,170.3,169.8,168.9,167.9,165.9,162.2,161.9,149.4,148.3,146.2,145.7$, 138.1, 128.2, 127.6, 127.2, 120.9, 119.7, 119.4, 118.8, 118.6, 117.9, 112.8, 110.2, 78.7, 73.3, 64.4, 59.1, 58.1, 57.1, 55.8, 51.6, 46.7, 38.5, 35.3, 35.1, 29.9, 29.4, 27.5, 26.0, 23.7, 20.5. HR-MS (ESI-TOF) $m / z$ for $\left[\mathrm{C}_{47} \mathrm{H}_{51} \mathrm{ClN}_{8} \mathrm{NaO}_{17} \mathrm{~S}\right]^{+}\left(\left[\mathrm{M}+\mathrm{Na}^{+}\right)\right.$: calculated 1089.2674, measured 1089.2656. 


\section{SUPPLEMENTAL REFERENCES}

S1. Joshua, A. V.; Sharma, S. K.; Abrams, D. N. Syn. Comm. 2008, 38, 3, 434

S2. Kim, J. Lee, J. E.; Ree, H; Kim, H. J. Bull. Kor. Chem. Soc. 2015, 36, 439

S3. Tibrewal, N.; Elliott, G. I. ChemMedChem, 2020, 15, 23, 2269

S4. Lorion, M. M.; Duarte, F. J. S.; Calhorda, M. J.; Oble, J.; Poli, G. Org. Lett. 2016, 18, 5, 1020

S5. Larsen, S. D.; Barf, T.; Liljebris, C.; May, P. D.; Ogg, D.; O’Sullivan, T. J.; Palazuk, B. J.; Schostarez, H. J.; Stevens, F. C.; Bleasdale, J. E. J. Med. Chem. 2002, 45, 3, 598 


\section{SPECTRAL DATA}

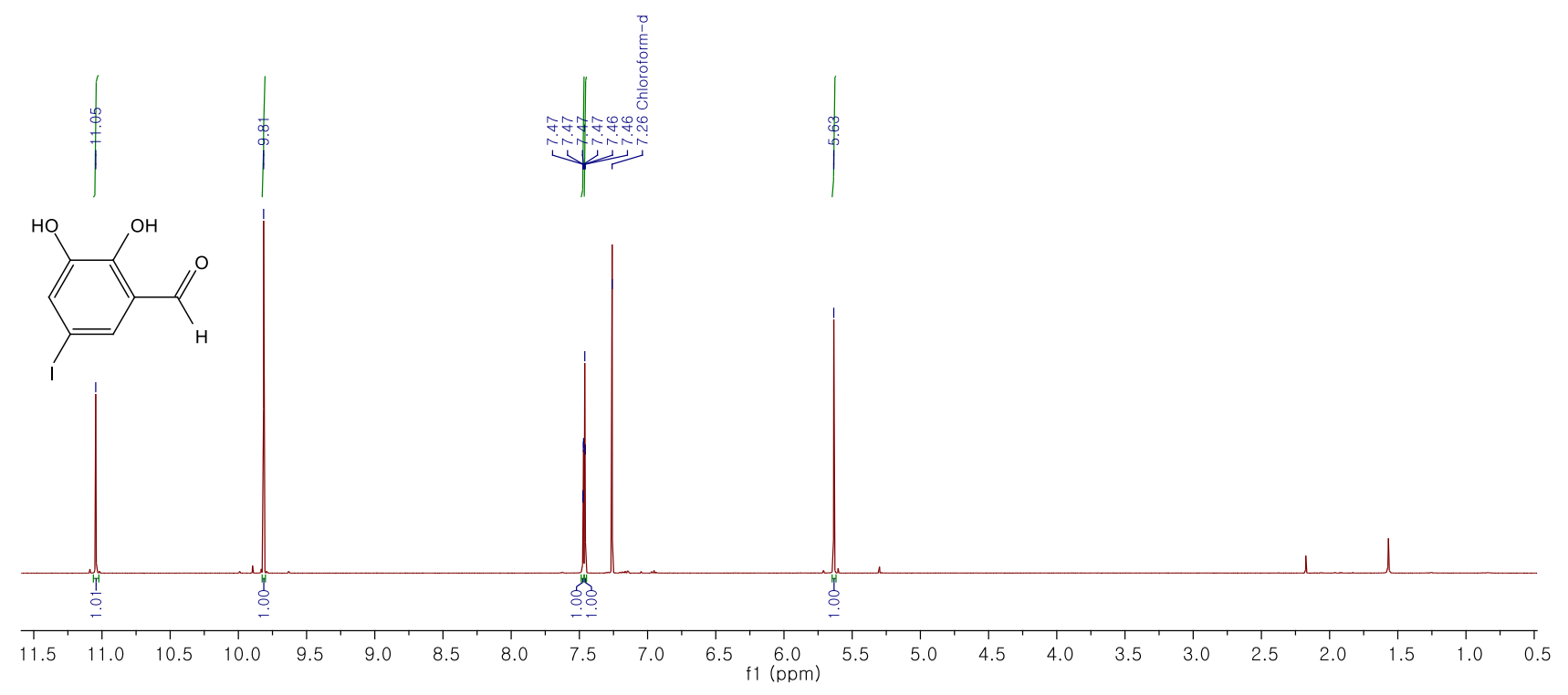

${ }^{1} \mathrm{H}-\mathrm{NMR}$ of compound $6\left(500 \mathrm{MHz}, \mathrm{CDCl}_{3}\right)$
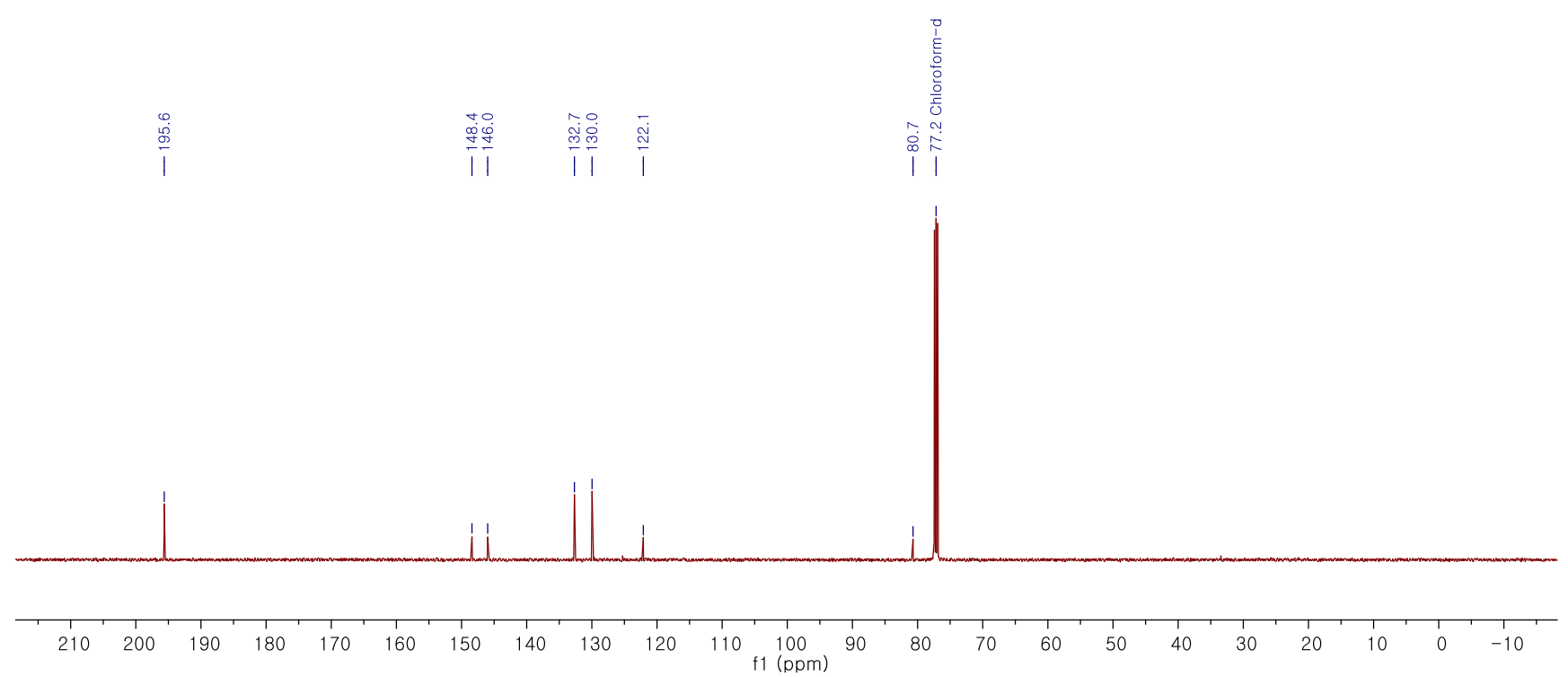

${ }^{13} \mathrm{C}$-NMR of compound $6\left(125 \mathrm{MHz}, \mathrm{CDCl}_{3}\right)$ 


\section{Compound Spectrum SmartFormula Report}

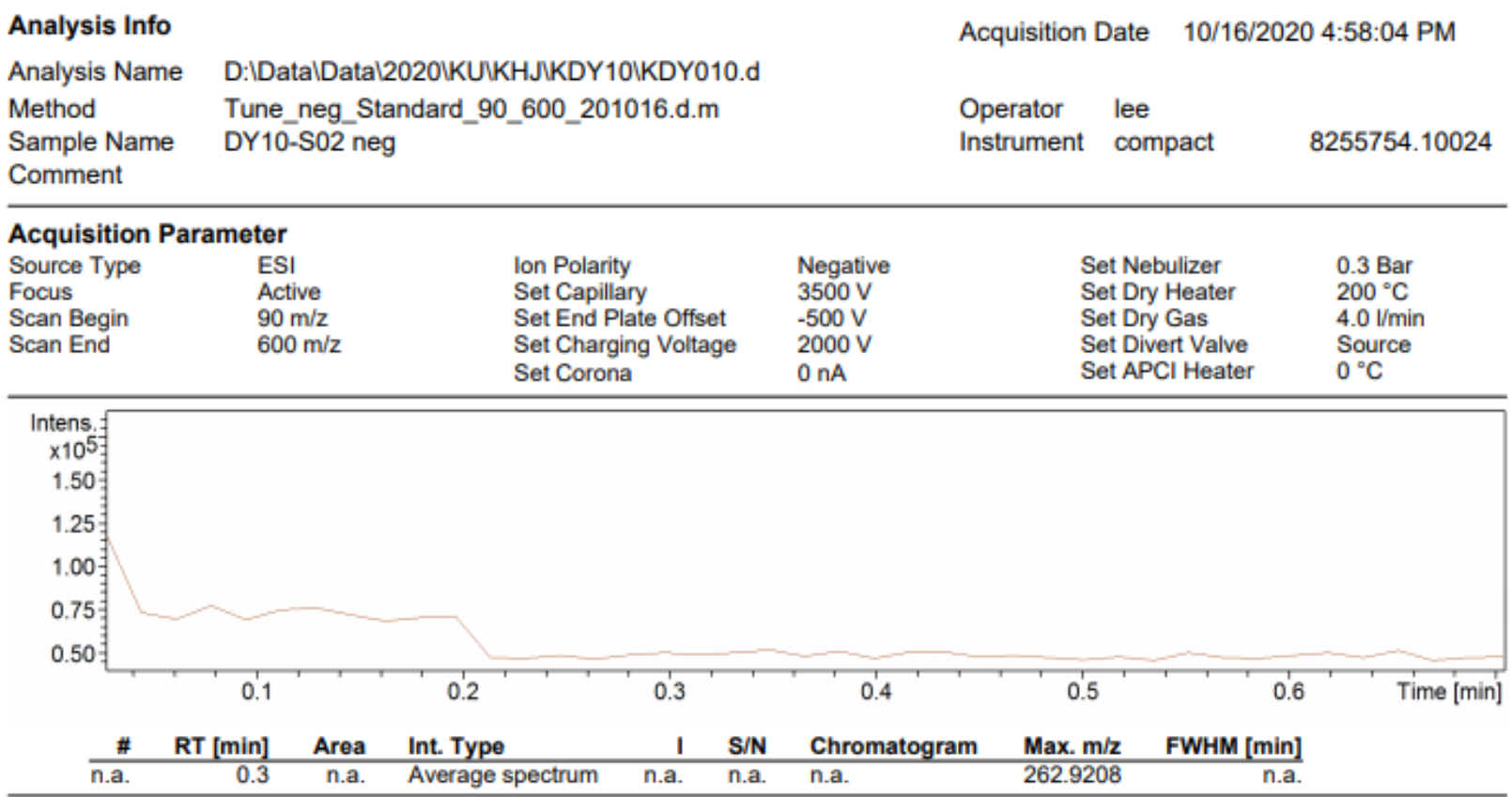

-MS, 0.1-0.6min \#5-32

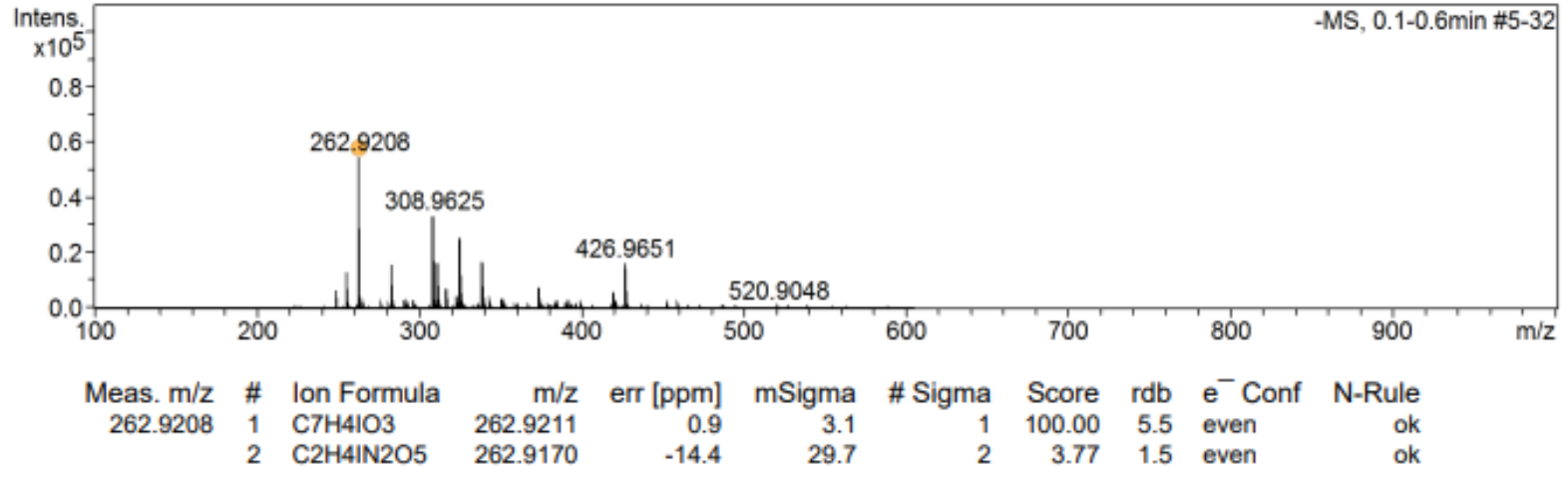

KDY010.d

HR-MS of compound 6 

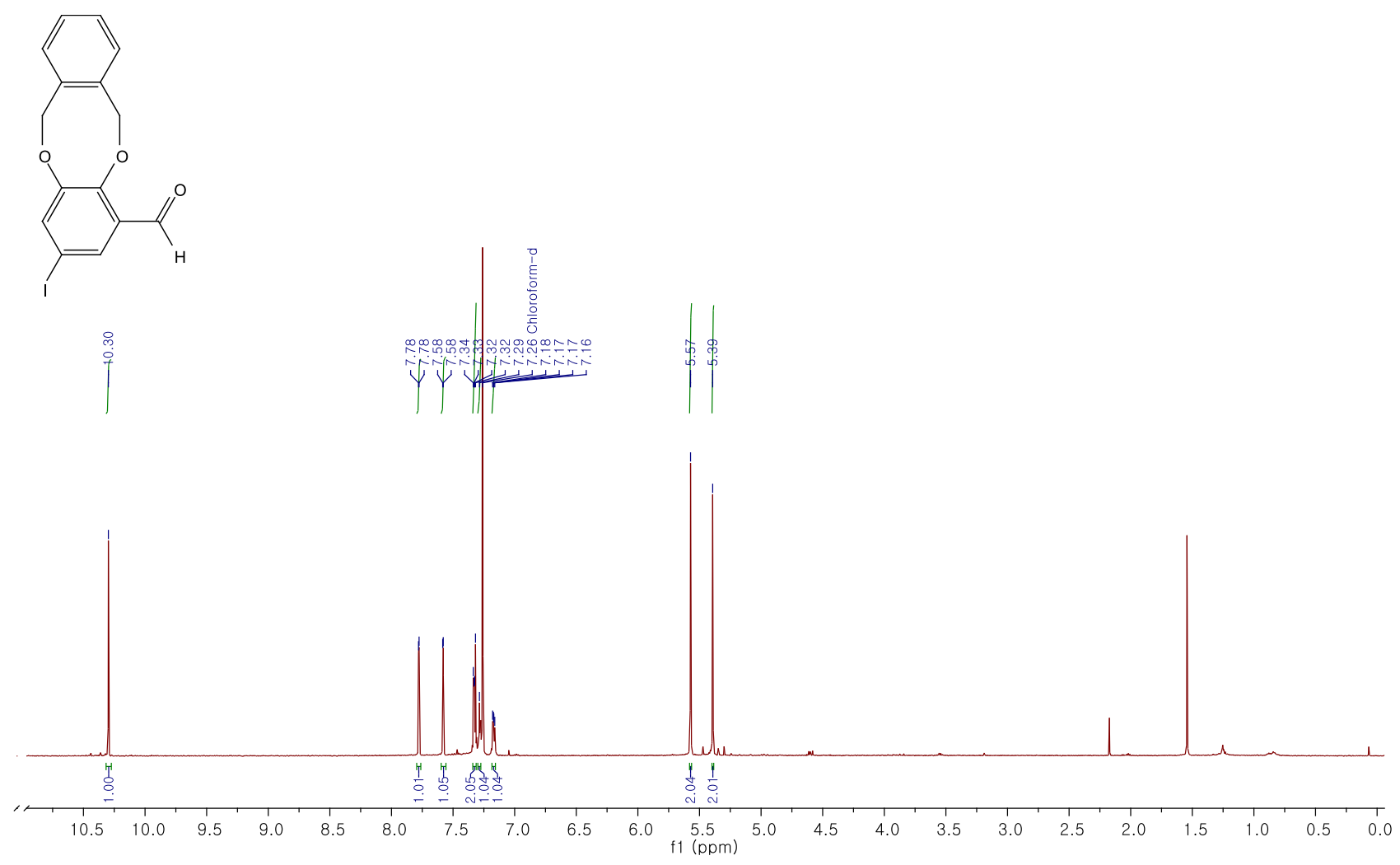

${ }^{1} \mathrm{H}-\mathrm{NMR}$ of compound $\mathbf{S 2}\left(500 \mathrm{MHz}, \mathrm{CDCl}_{3}\right)$
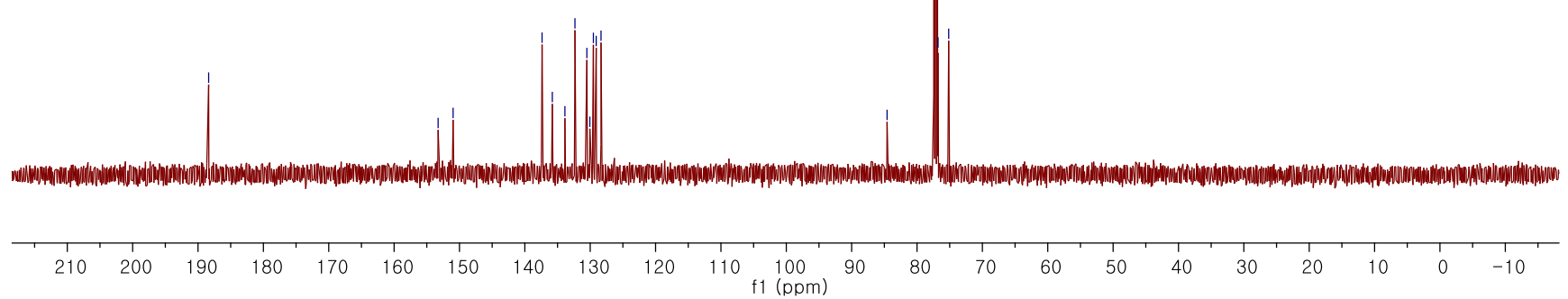

${ }^{13} \mathrm{C}$-NMR of compound $\mathbf{S 2}\left(125 \mathrm{MHz}, \mathrm{CDCl}_{3}\right)$ 


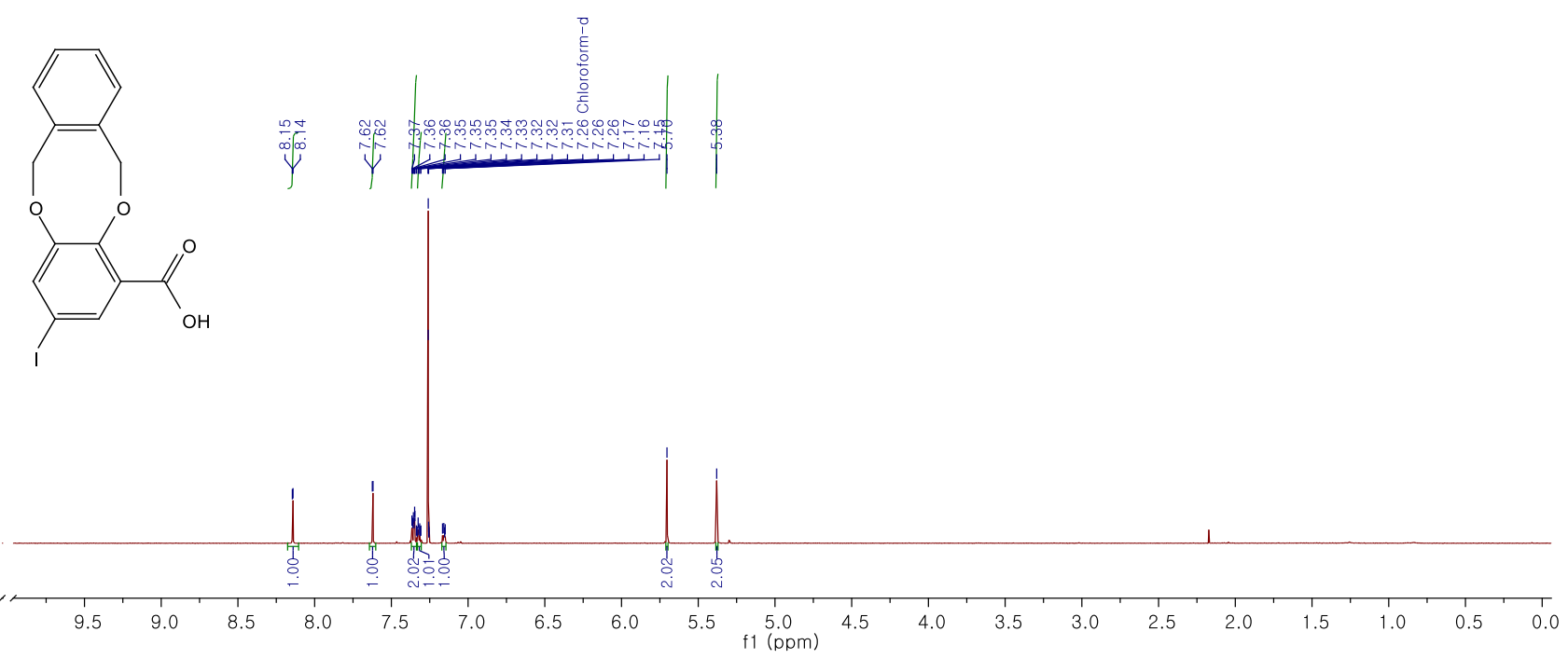

${ }^{1} \mathrm{H}-\mathrm{NMR}$ of compound $7 \mathbf{a}\left(500 \mathrm{MHz}, \mathrm{CDCl}_{3}\right)$

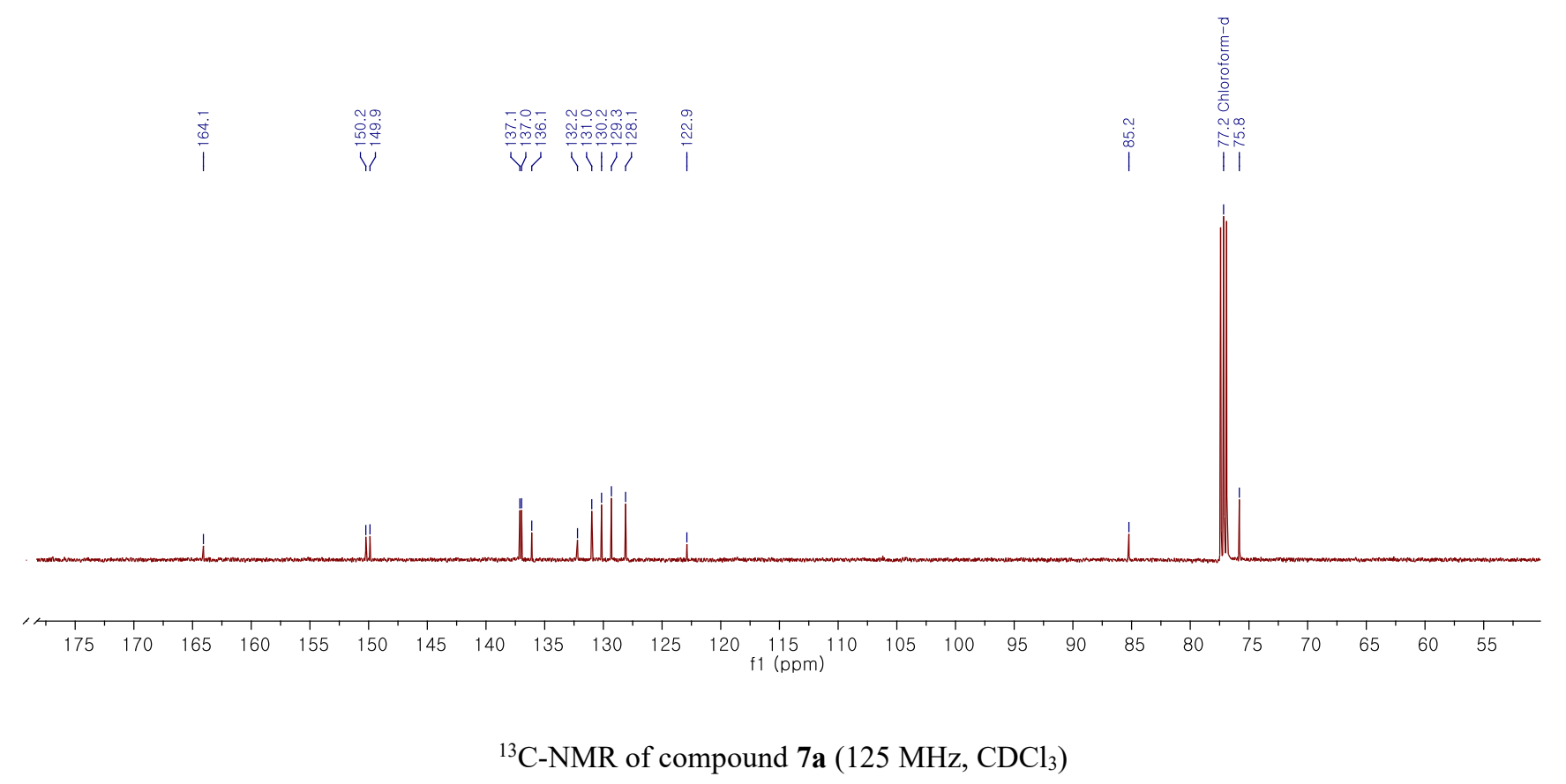




\section{Compound Spectrum SmartFormula Report}

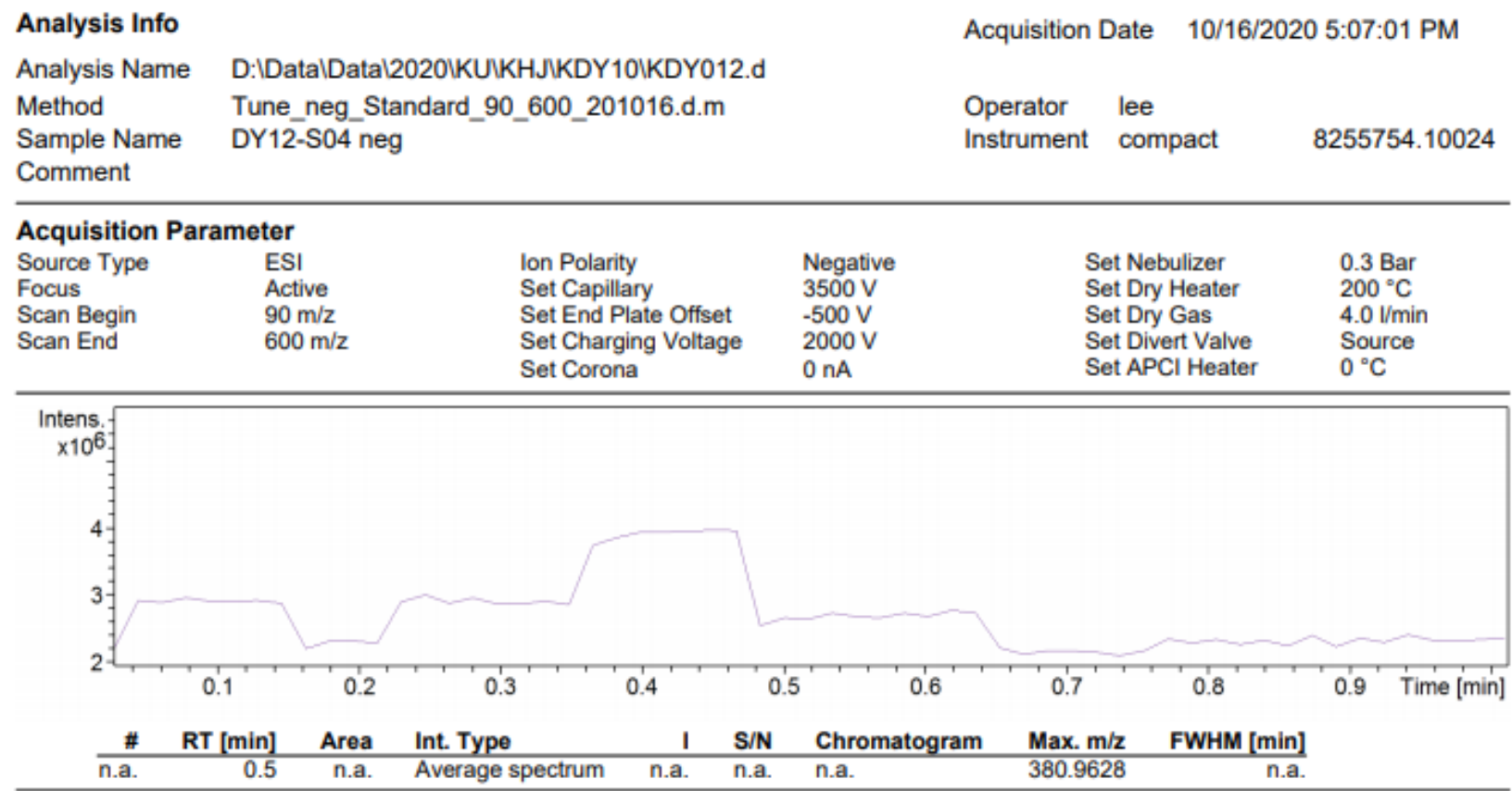

-MS, 0.2-0.8min \#13-44

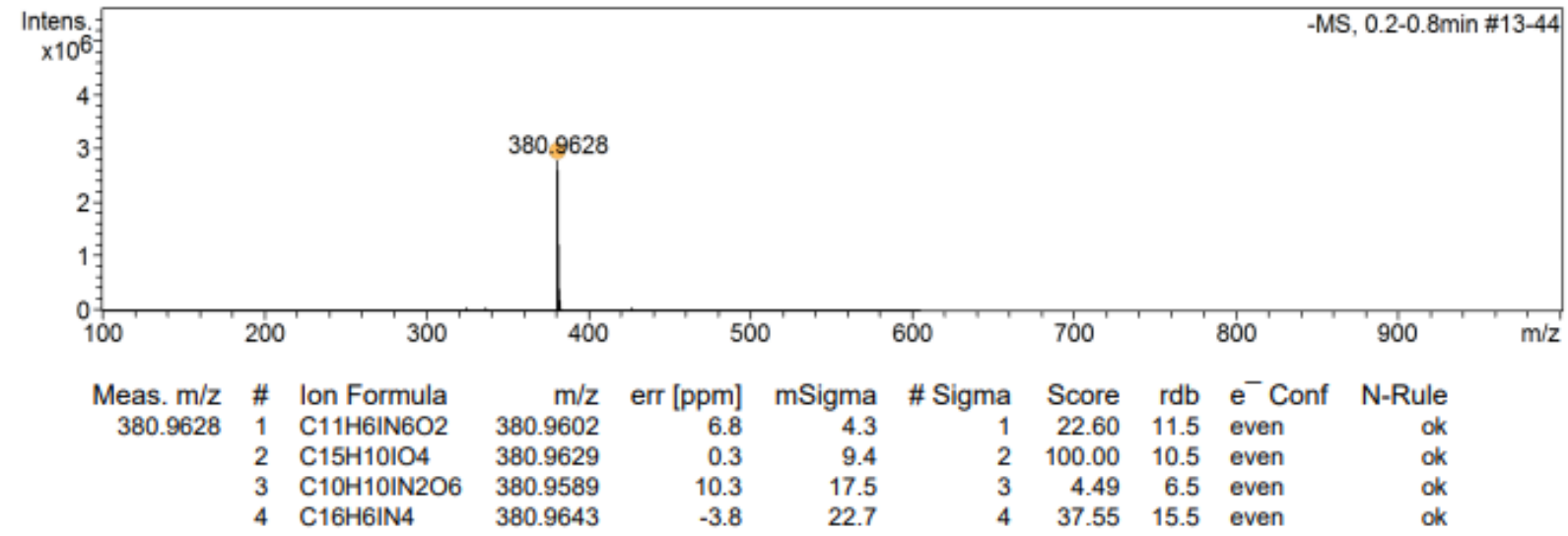

KDY012.d

Bruker Compass DataAnalysis 4.1 $\quad$ printed: $10 / 19 / 2020$ 11:12:42 AM $\quad$ by: lee $\quad$ Page 1 of 1

HR-MS of compound 7a 


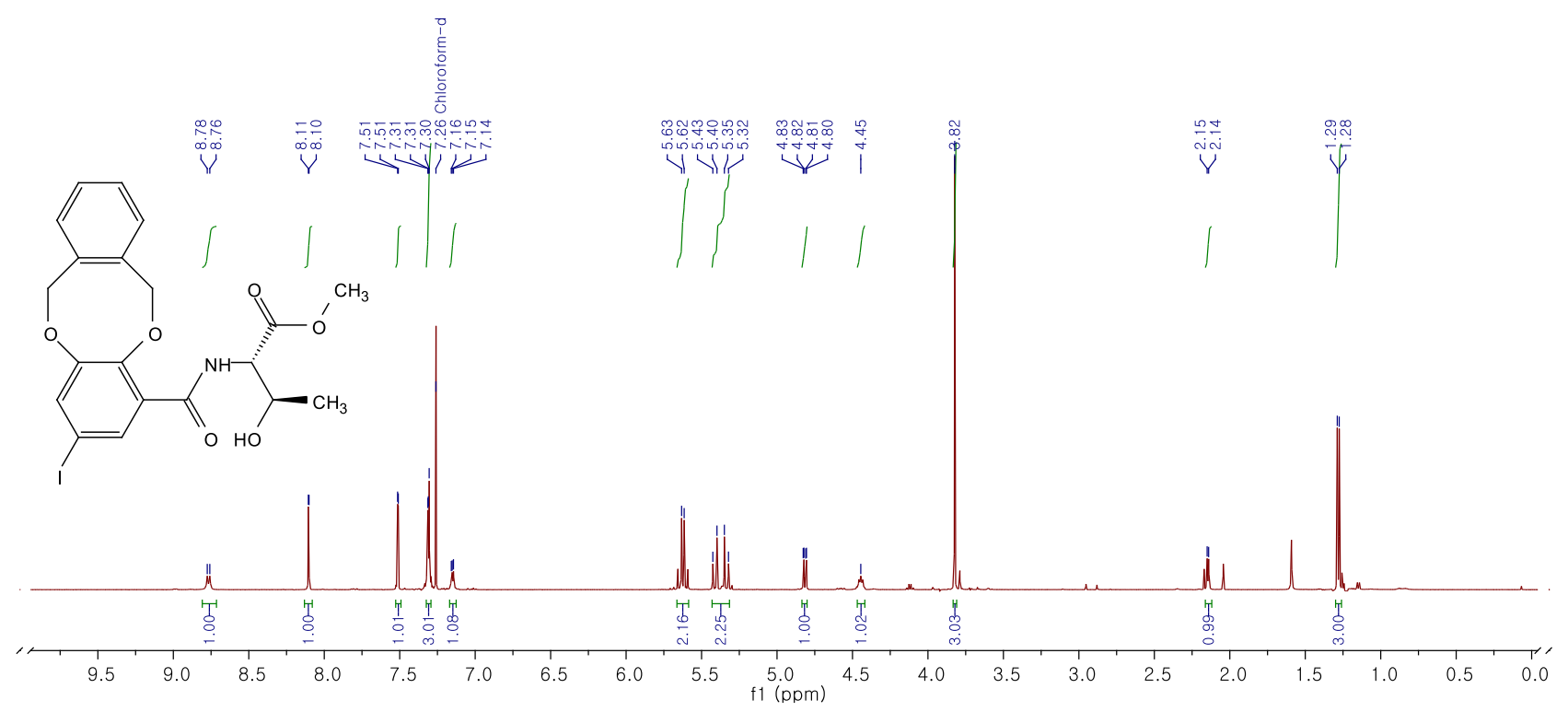

${ }^{1} \mathrm{H}-\mathrm{NMR}$ of compound $\mathbf{S 3}\left(500 \mathrm{MHz}, \mathrm{CDCl}_{3}\right)$

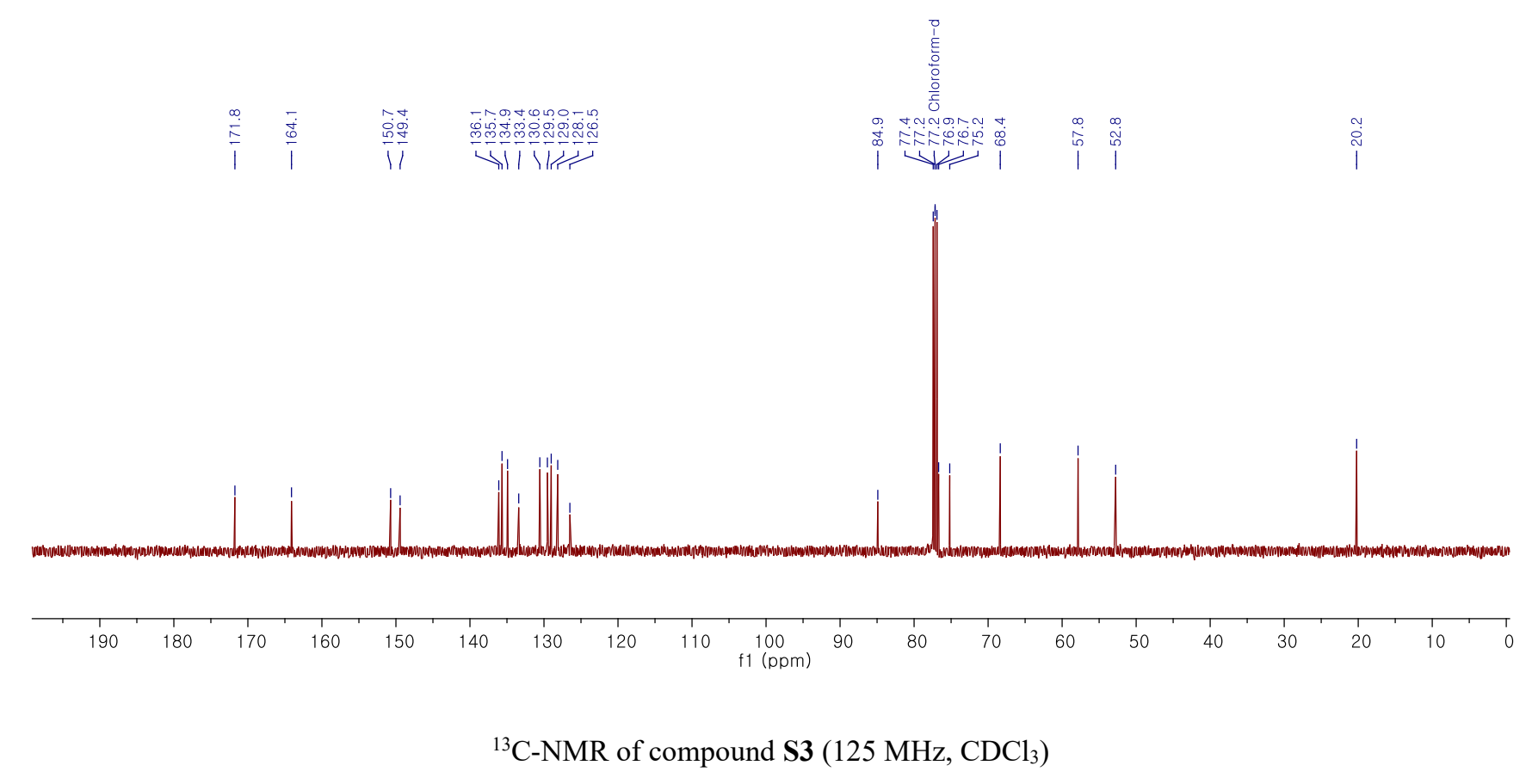




\section{Compound Spectrum SmartFormula Report}

\section{Analysis Info}

Analysis Name

Method

Sample Name

Comment
Acquisition Date $\quad$ 10/16/2020 3:27:59 PM

Operator lee

Instrument compact 8255754.10024

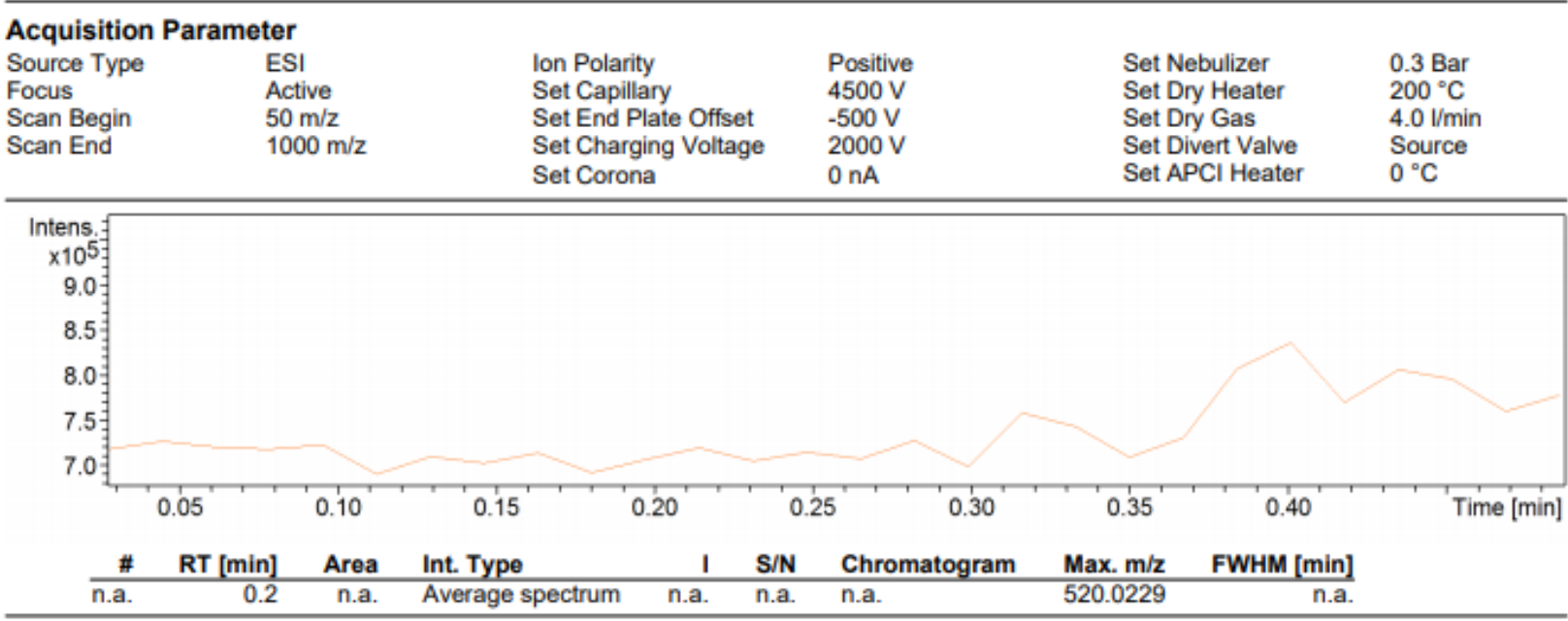

+MS, 0.1-0.4min \#6-21

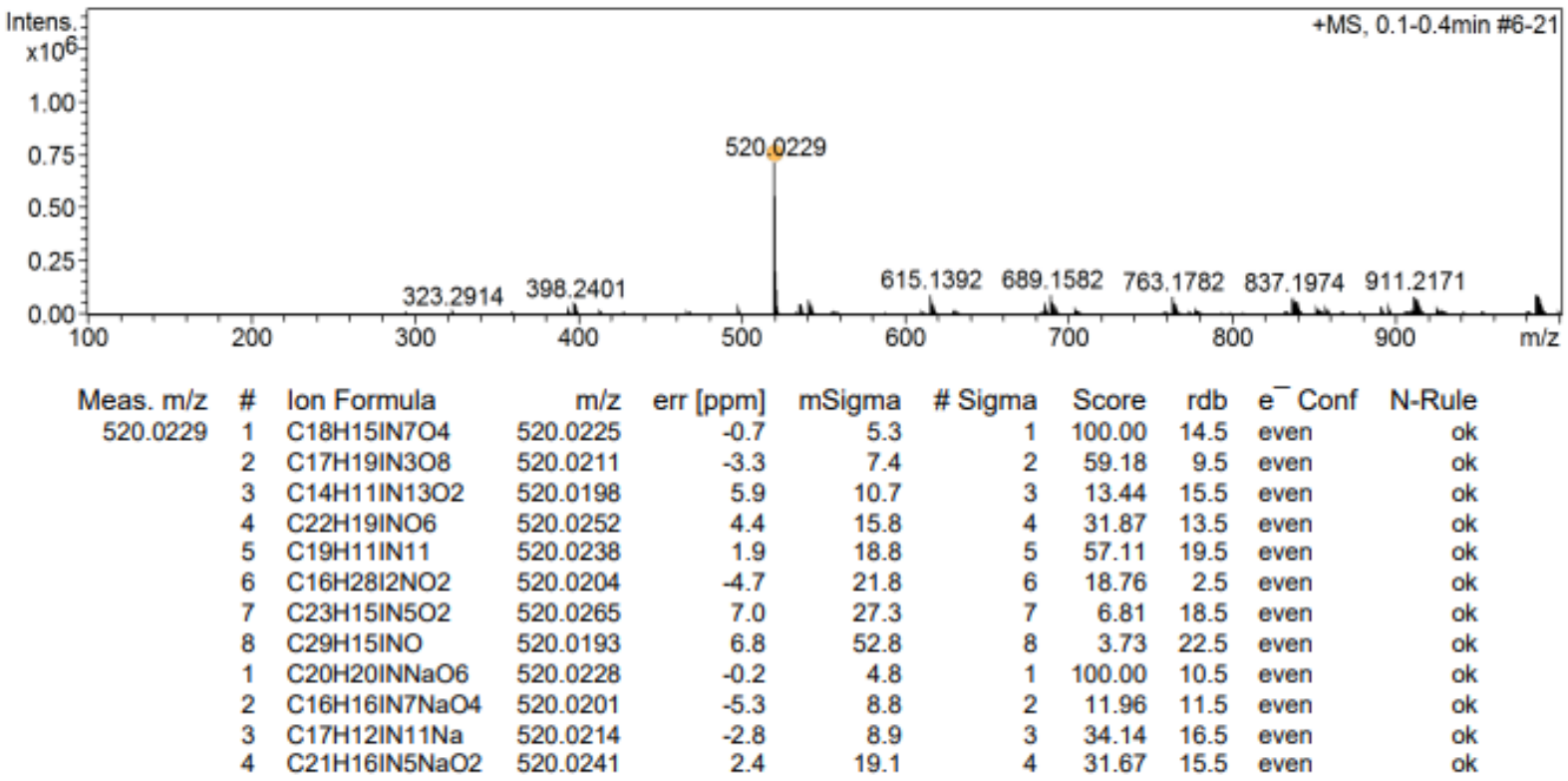

KDY013.d

HR-MS of compound $\mathbf{S 3}$ 


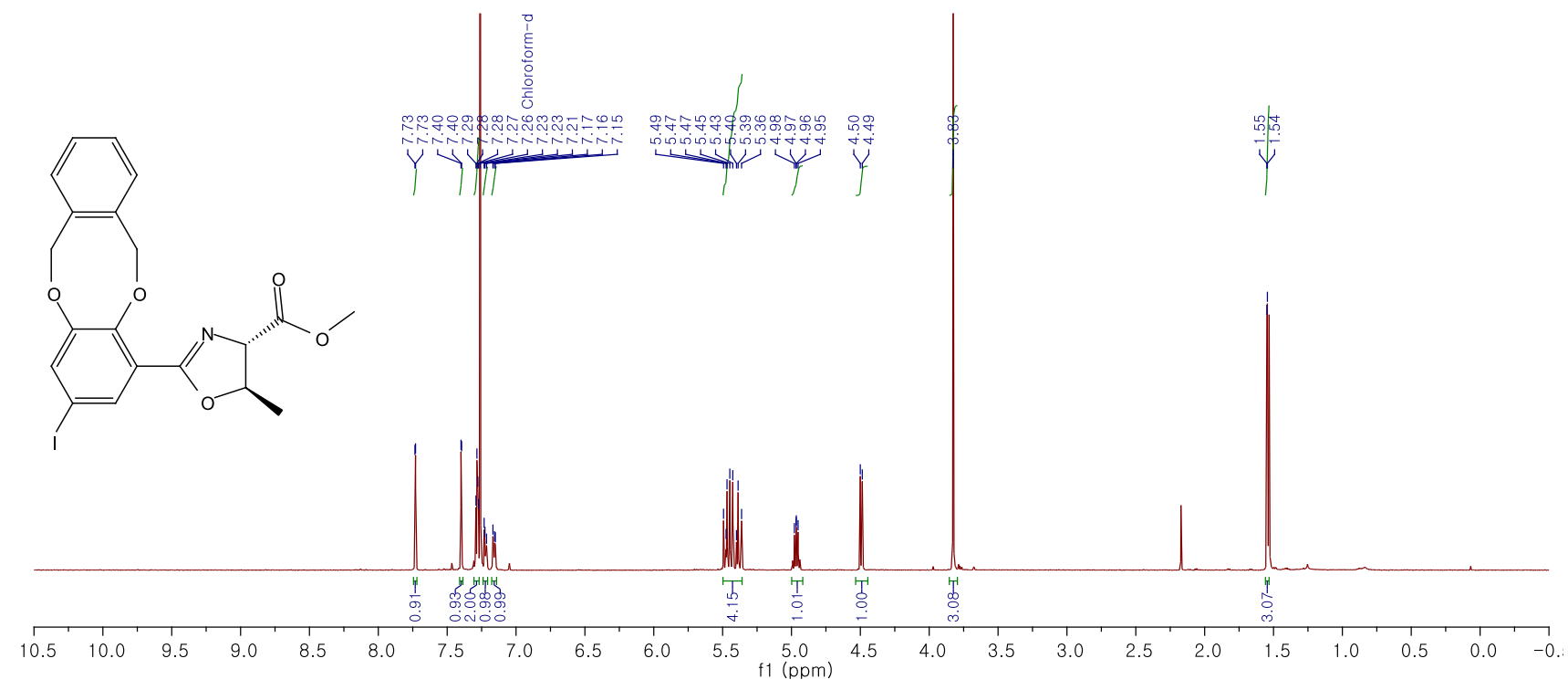

${ }^{1} \mathrm{H}-\mathrm{NMR}$ of compound $\mathbf{S 4}\left(500 \mathrm{MHz}, \mathrm{CDCl}_{3}\right)$
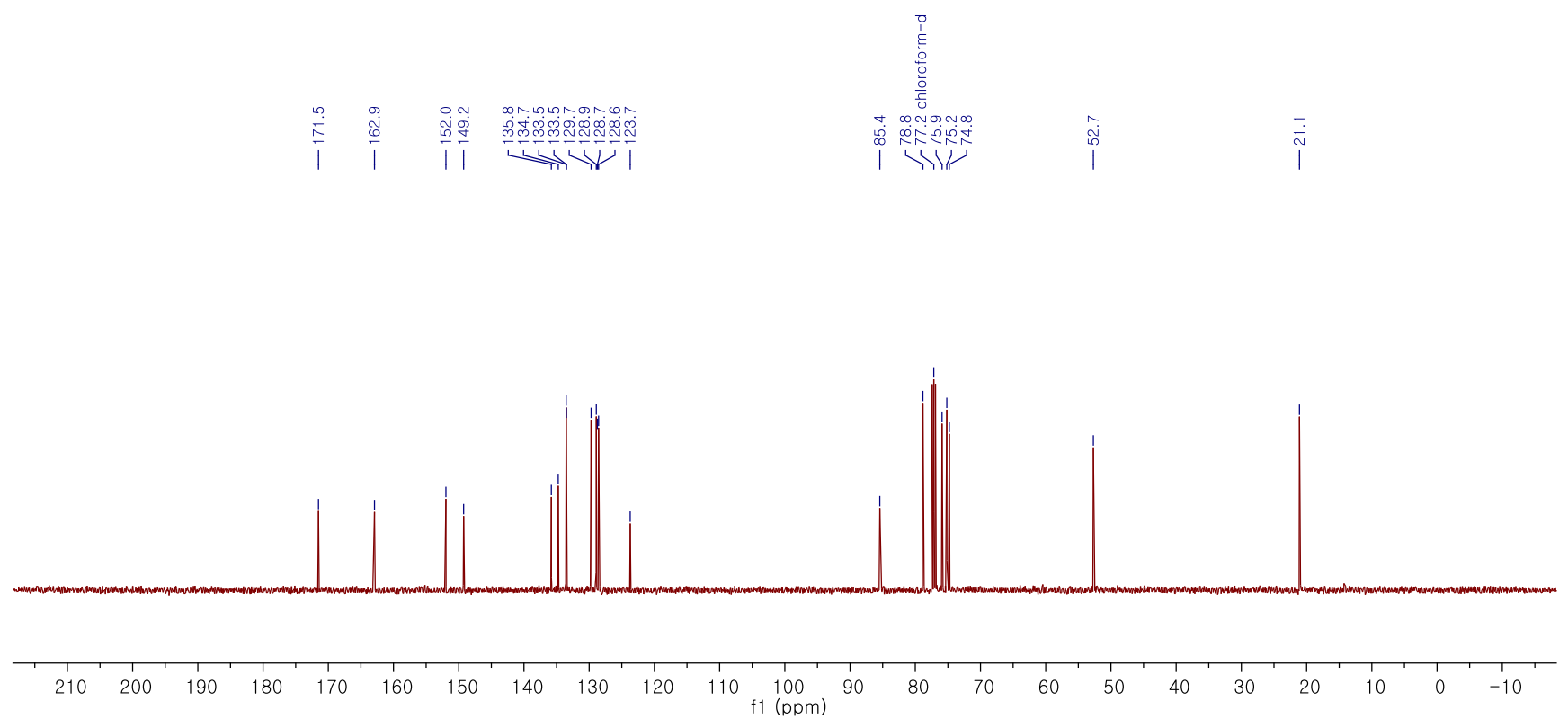

${ }^{13} \mathrm{C}$-NMR of compound $\mathbf{S 4}\left(125 \mathrm{MHz}, \mathrm{CDCl}_{3}\right)$ 


\section{Compound Spectrum SmartFormula Report}

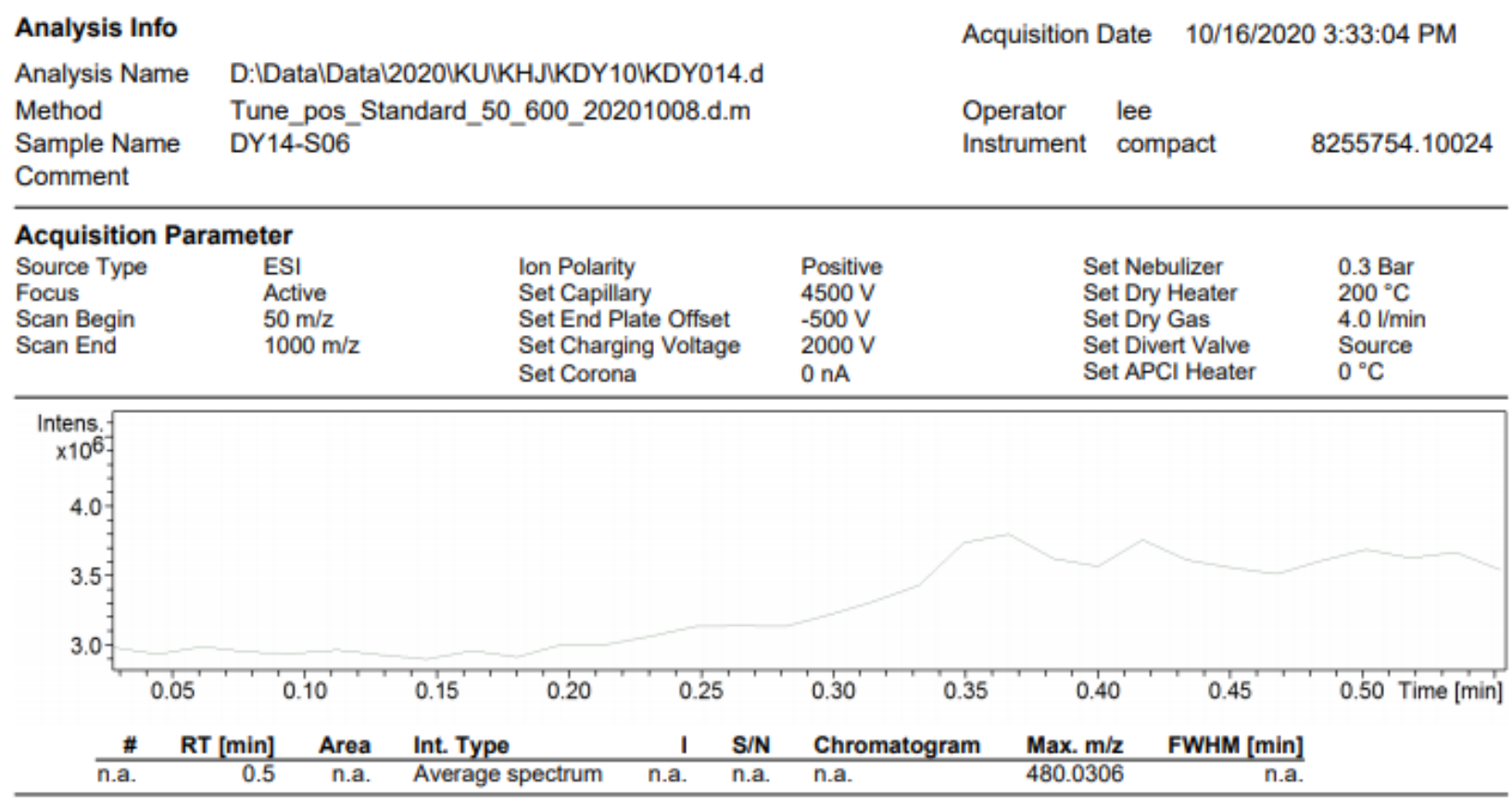

+MS, 0.4-0.5min \#24-31

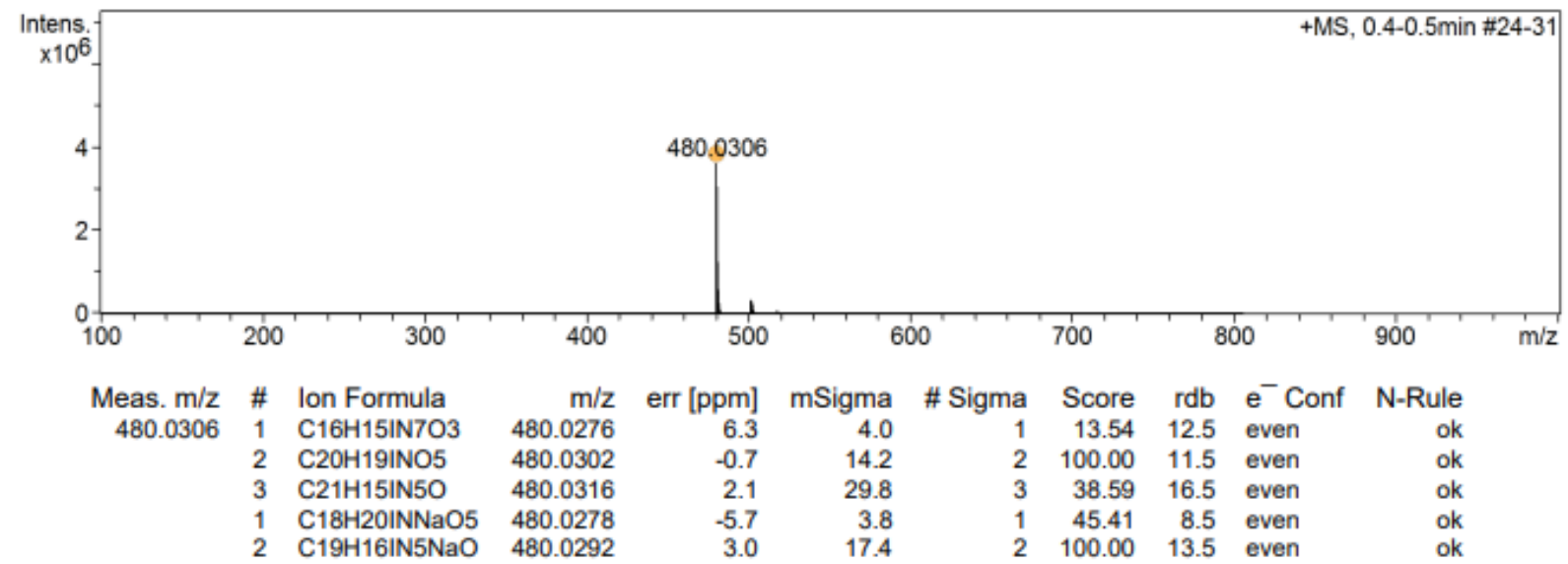

HR-MS of compound S4 


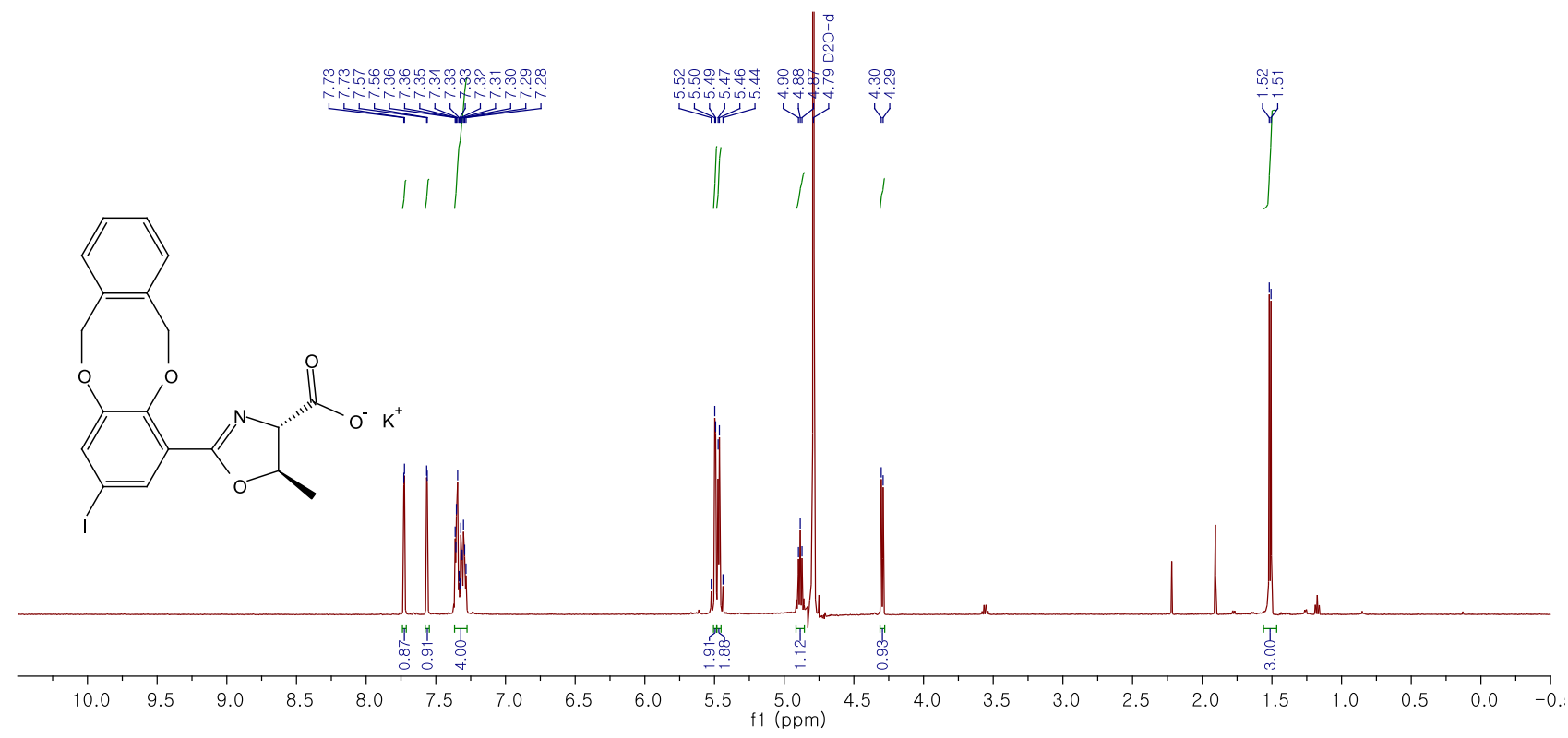

${ }^{1} \mathrm{H}-\mathrm{NMR}$ of compound $\mathbf{8 a}\left(500 \mathrm{MHz}, \mathrm{D}_{2} \mathrm{O}\right)$
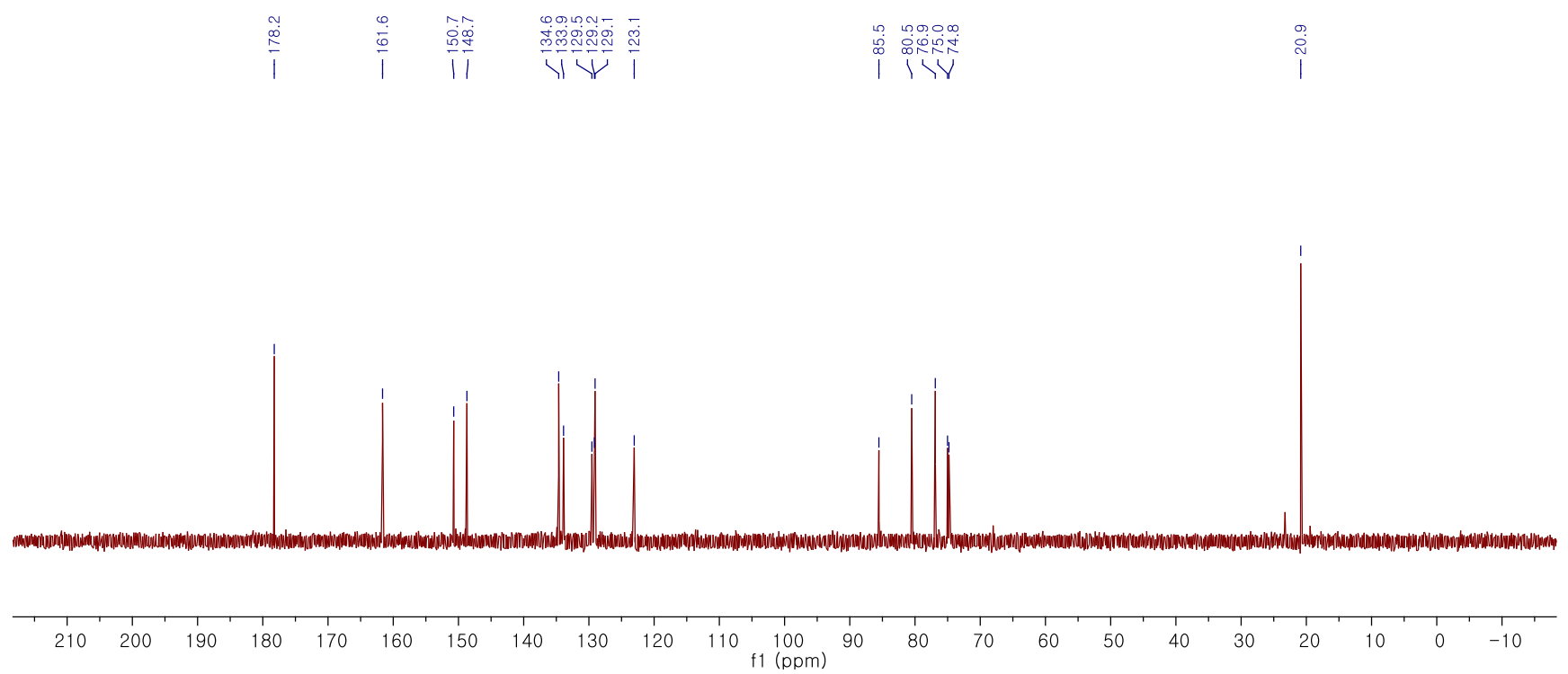

${ }^{13} \mathrm{C}-\mathrm{NMR}$ of compound $\mathbf{8 a}\left(125 \mathrm{MHz}, \mathrm{D}_{2} \mathrm{O}\right)$ 


\section{Compound Spectrum SmartFormula Report}

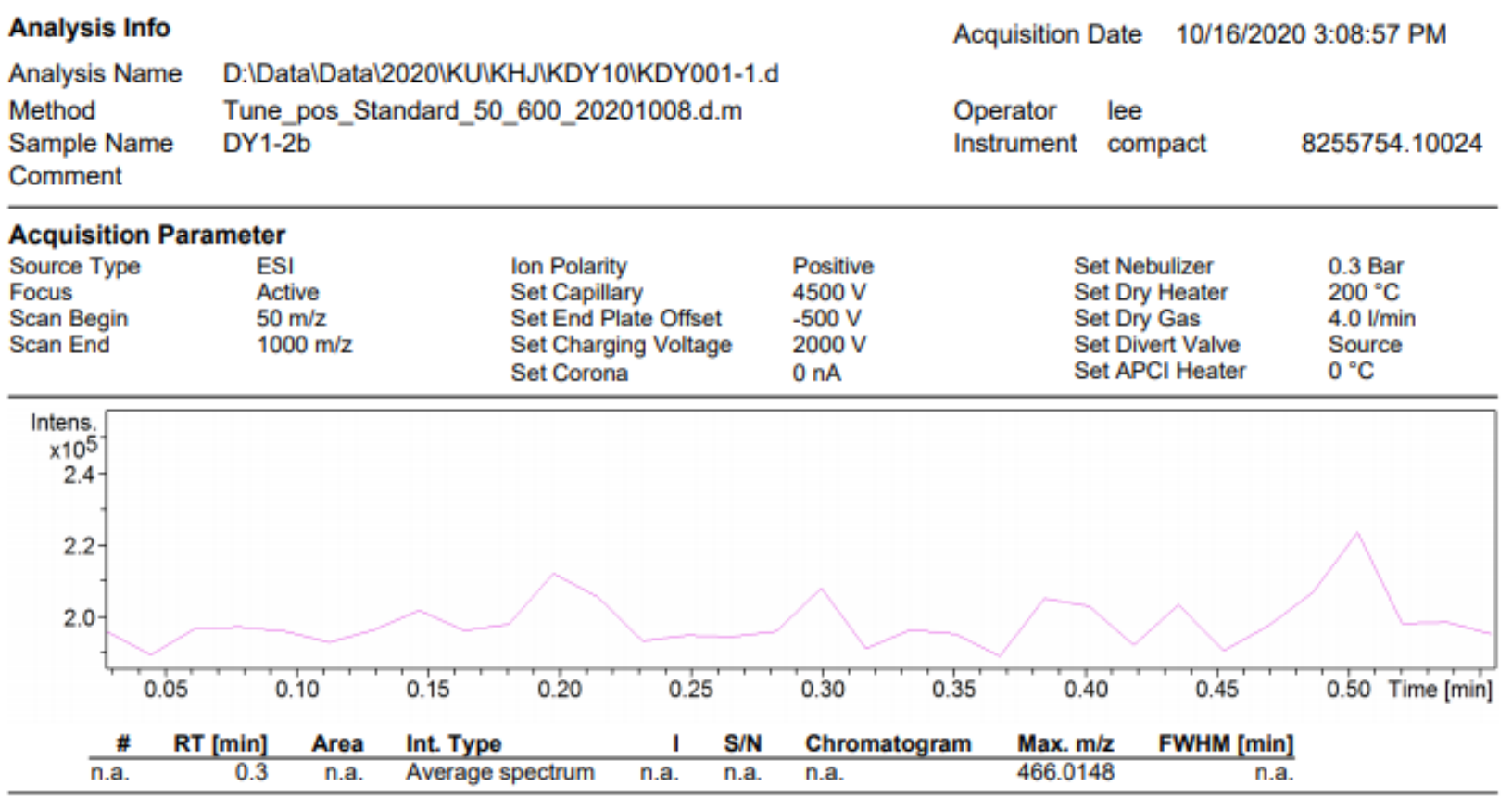

+MS, 0.1-0.4min \#7-24

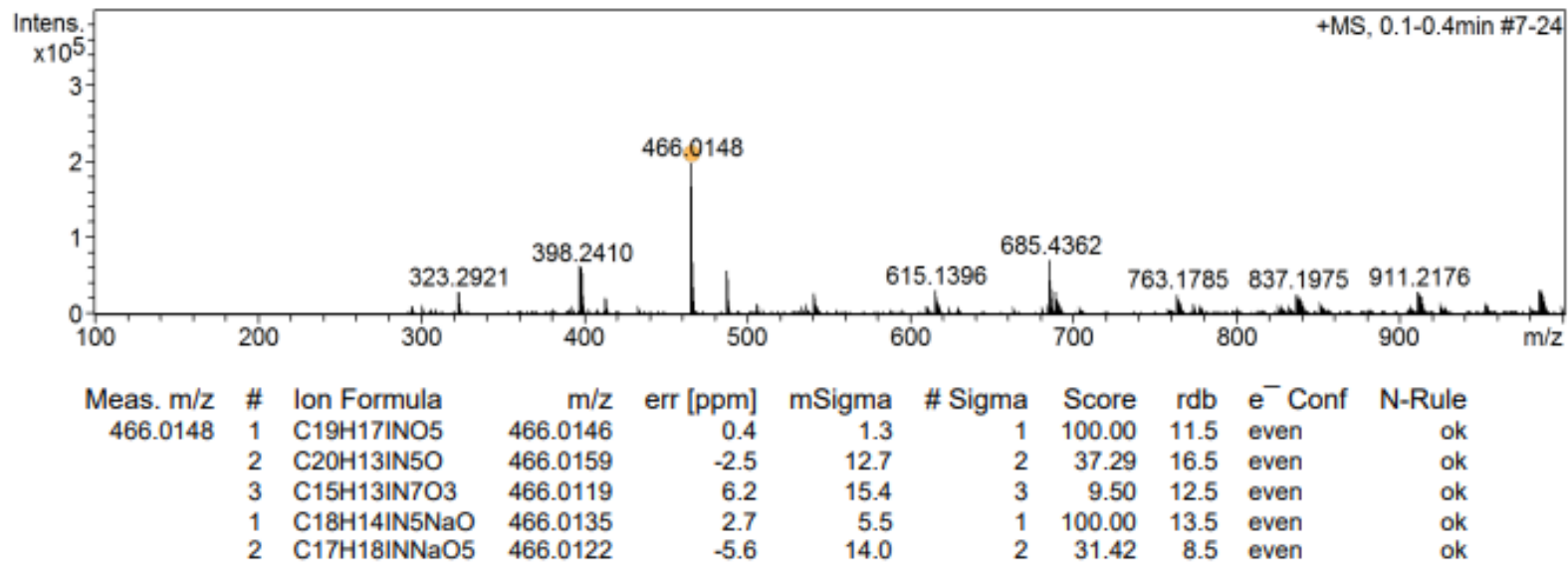

KDY001-1.d

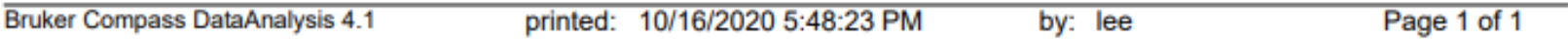

HR-MS of compound $\mathbf{8 a}$ 


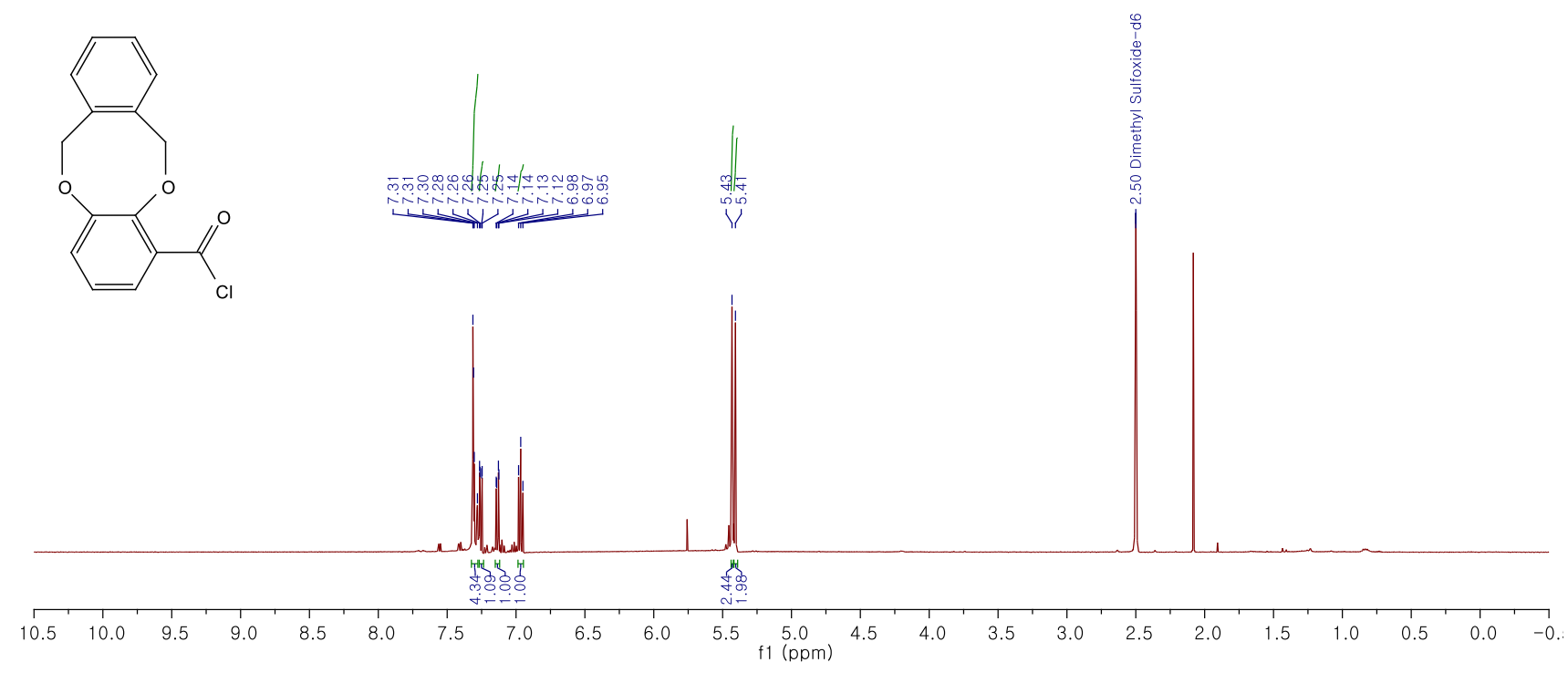

${ }^{1} \mathrm{H}-\mathrm{NMR}$ of compound $\mathbf{1 2 b}$ (500 MHz, DMSO-d ${ }^{6}$ )

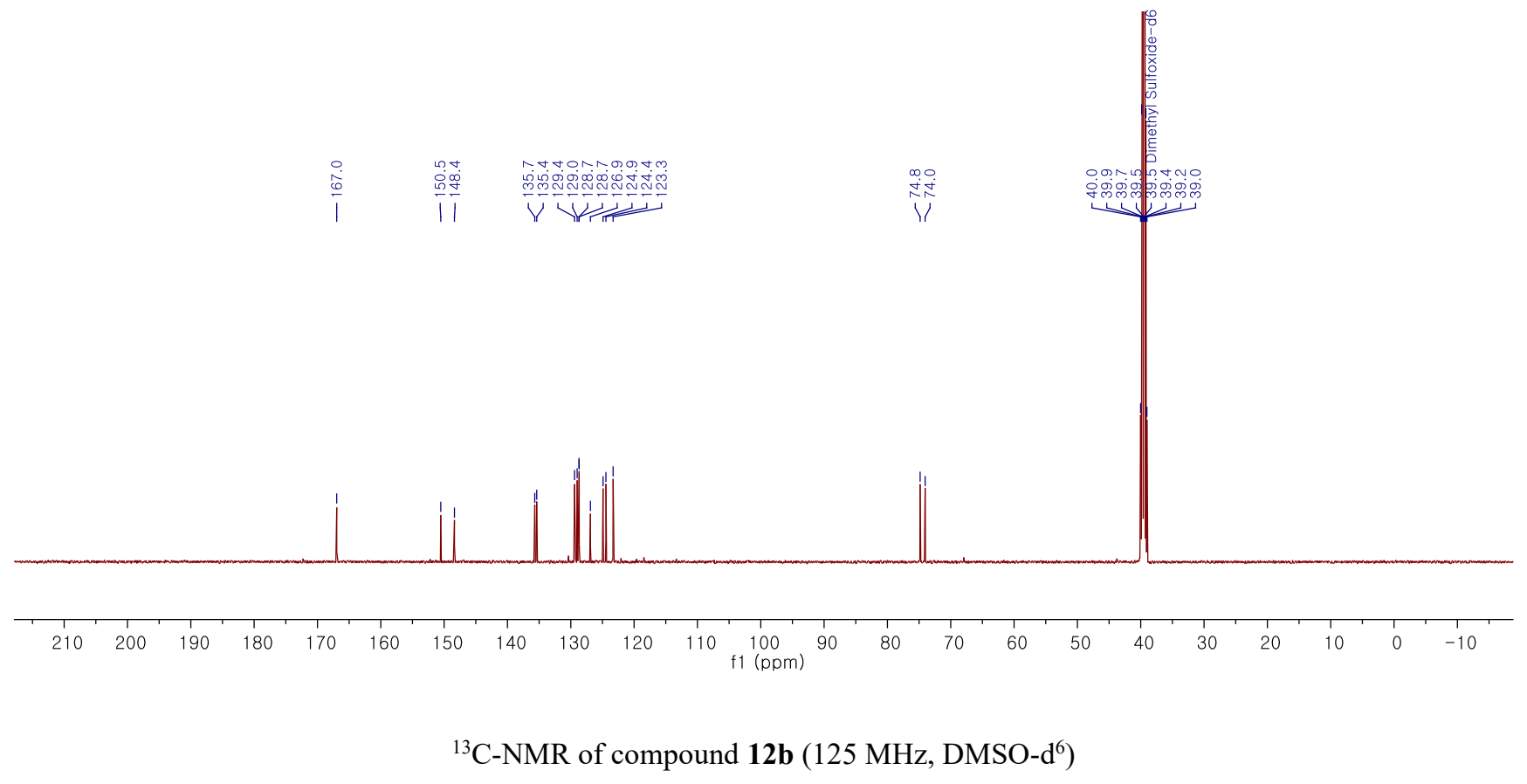




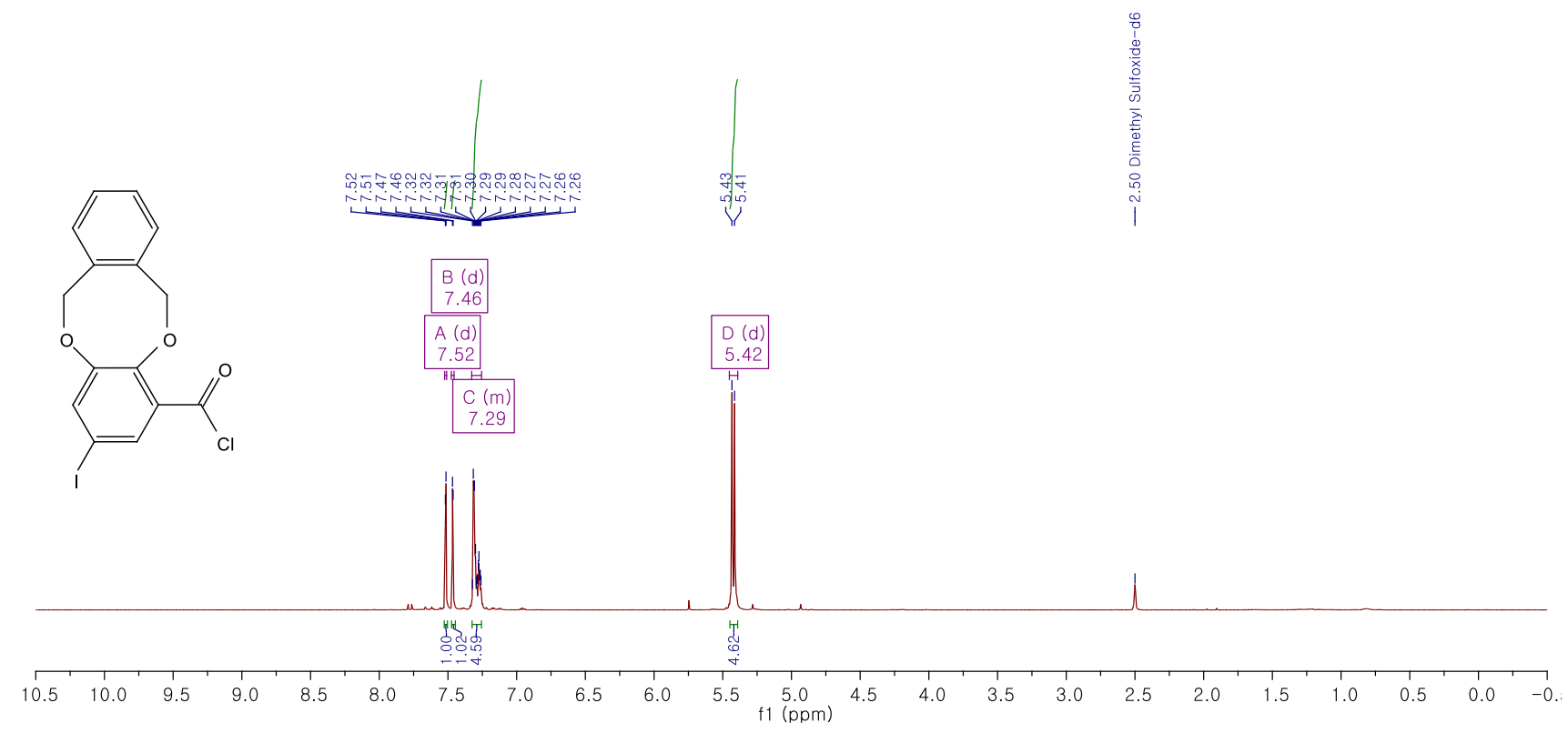

${ }^{1} \mathrm{H}-\mathrm{NMR}$ of compound 12a (500 MHz, DMSO-d ${ }^{6}$ )

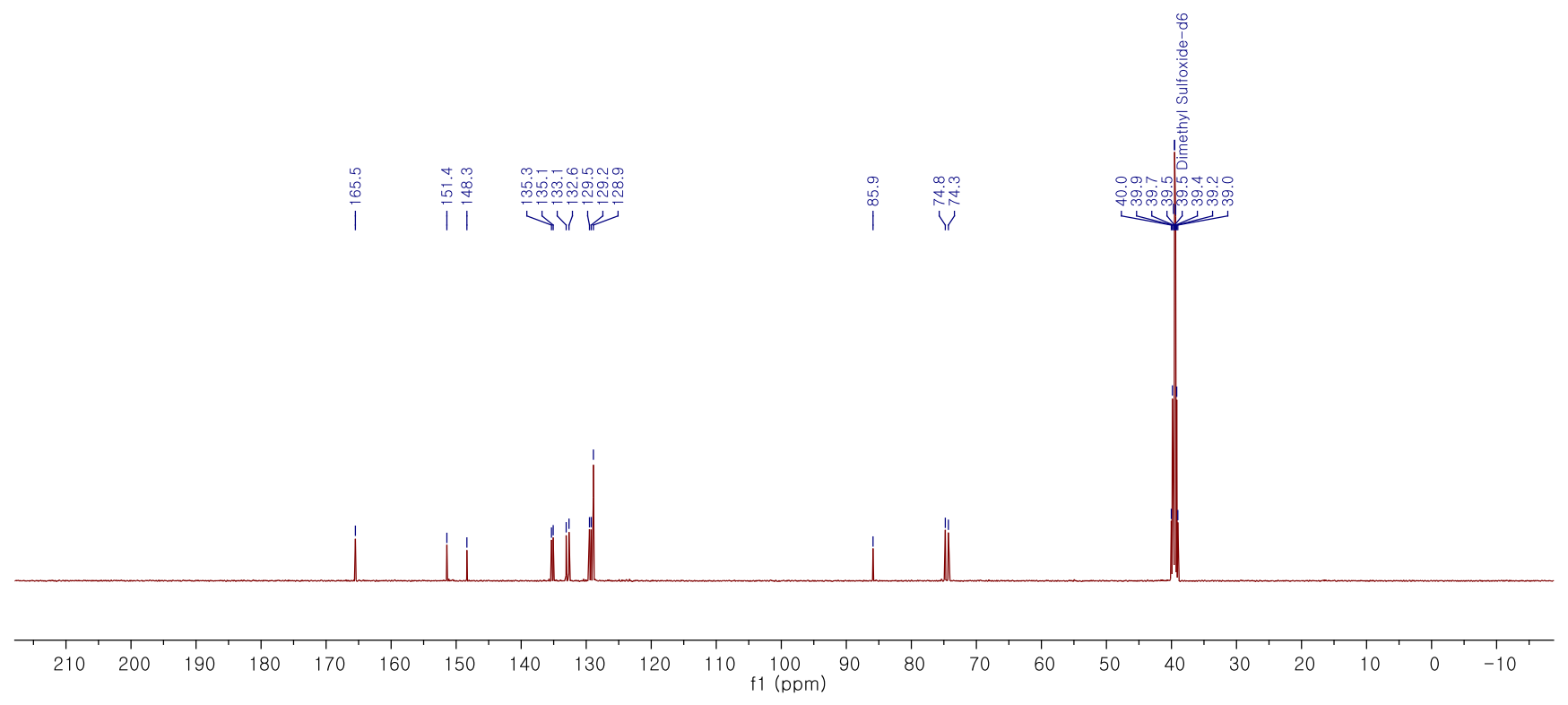

${ }^{13} \mathrm{C}-\mathrm{NMR}$ of compound 12a (125 MHz, DMSO-d ${ }^{6}$ ) 


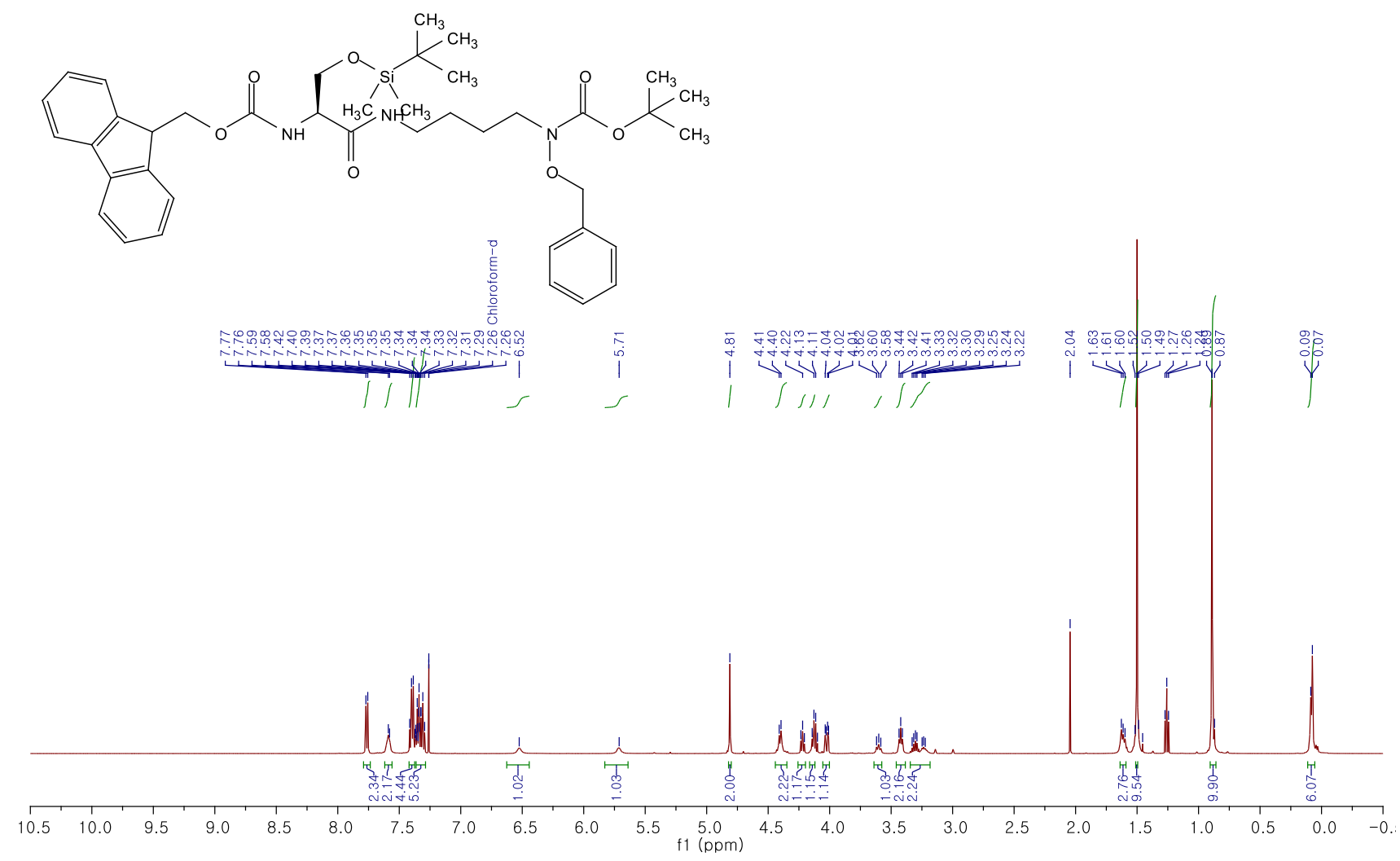

${ }^{1} \mathrm{H}-\mathrm{NMR}$ of compound $\mathbf{S 5}\left(500 \mathrm{MHz}, \mathrm{CDCl}_{3}\right)$

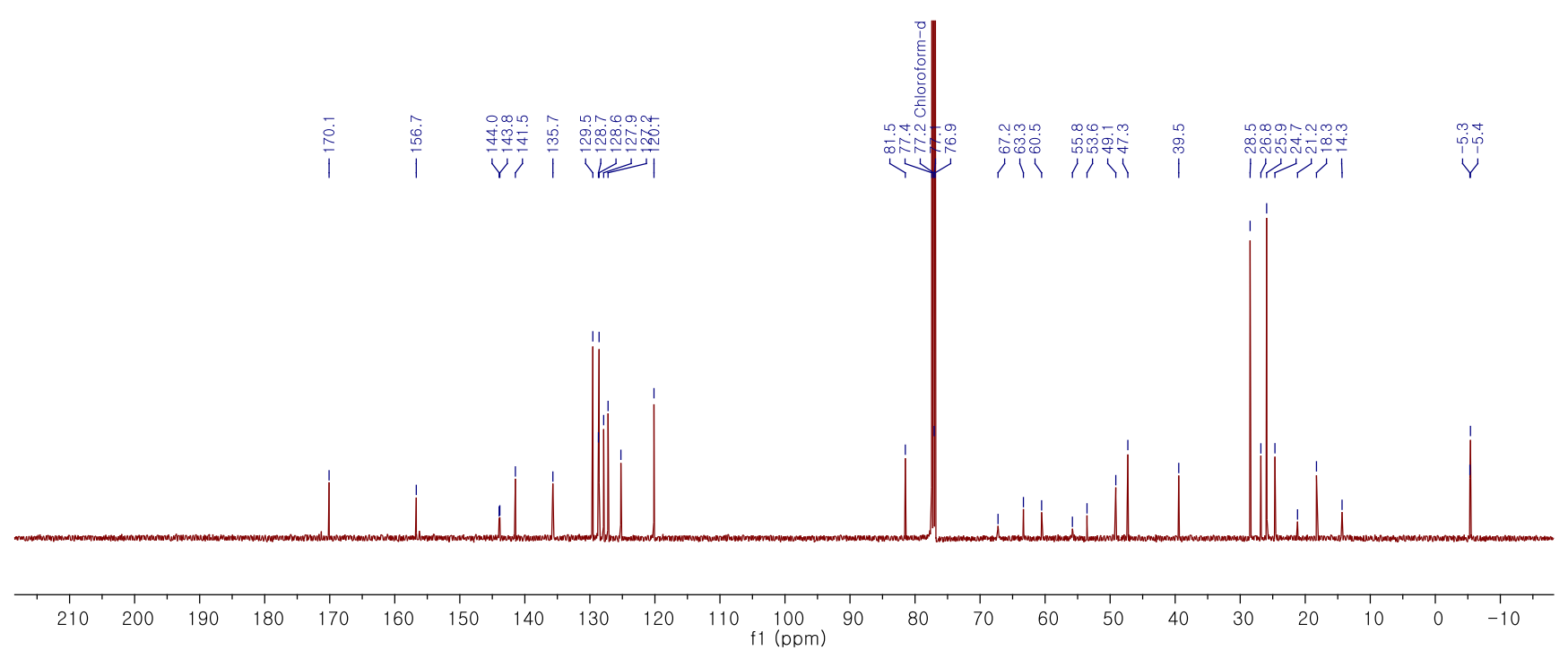

${ }^{13} \mathrm{C}$-NMR of compound $\mathbf{S 5}\left(125 \mathrm{MHz}, \mathrm{CDCl}_{3}\right.$ ) 


\section{Compound Spectrum SmartFormula Report}

\section{Analysis Info}

Analysis Name

Method

Sample Name

Comment
Acquisition Date 10/16/2020 3:36:25 PM

D:IDatalDatal2020IKUIKHJKDY10【KDY015.d

Tune_pos_Standard_50_600_20201008.d.m

DY15-S08
Operator lee

Instrument compact
8255754.10024

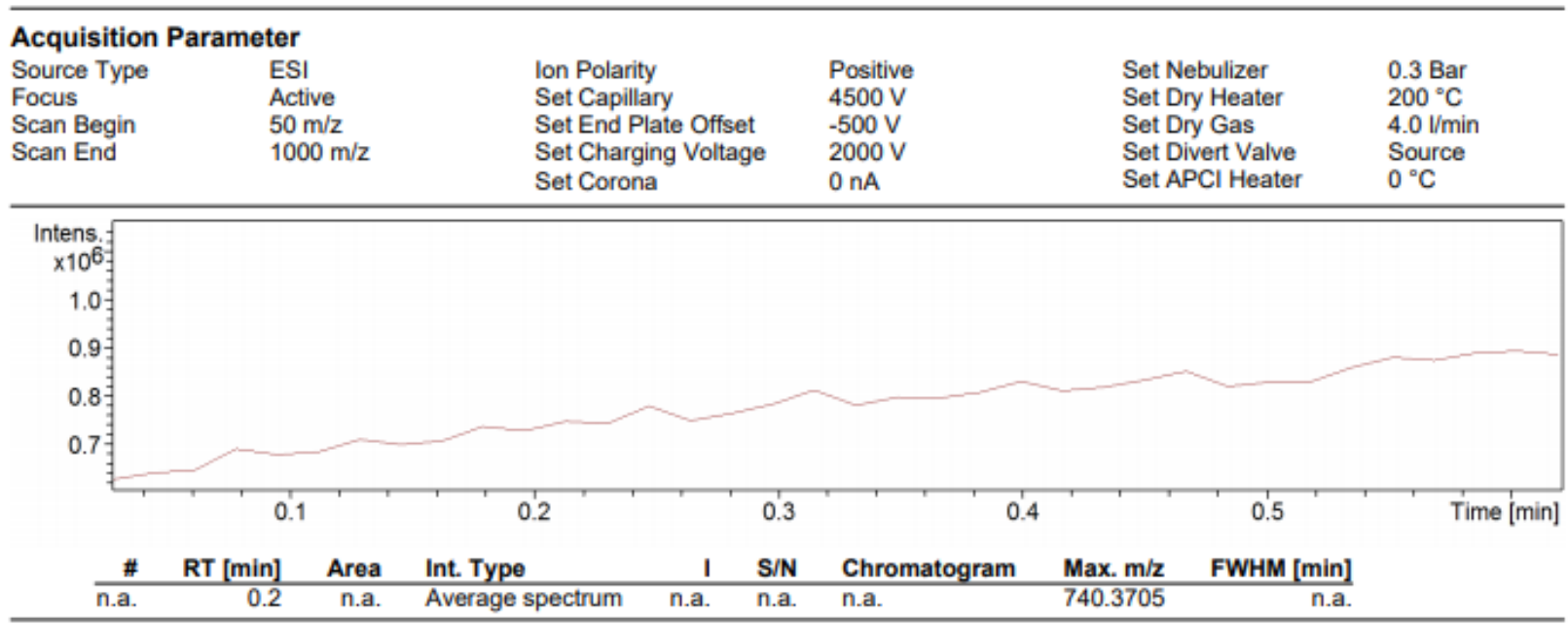

+MS, 0.1-0.2min \#5-14

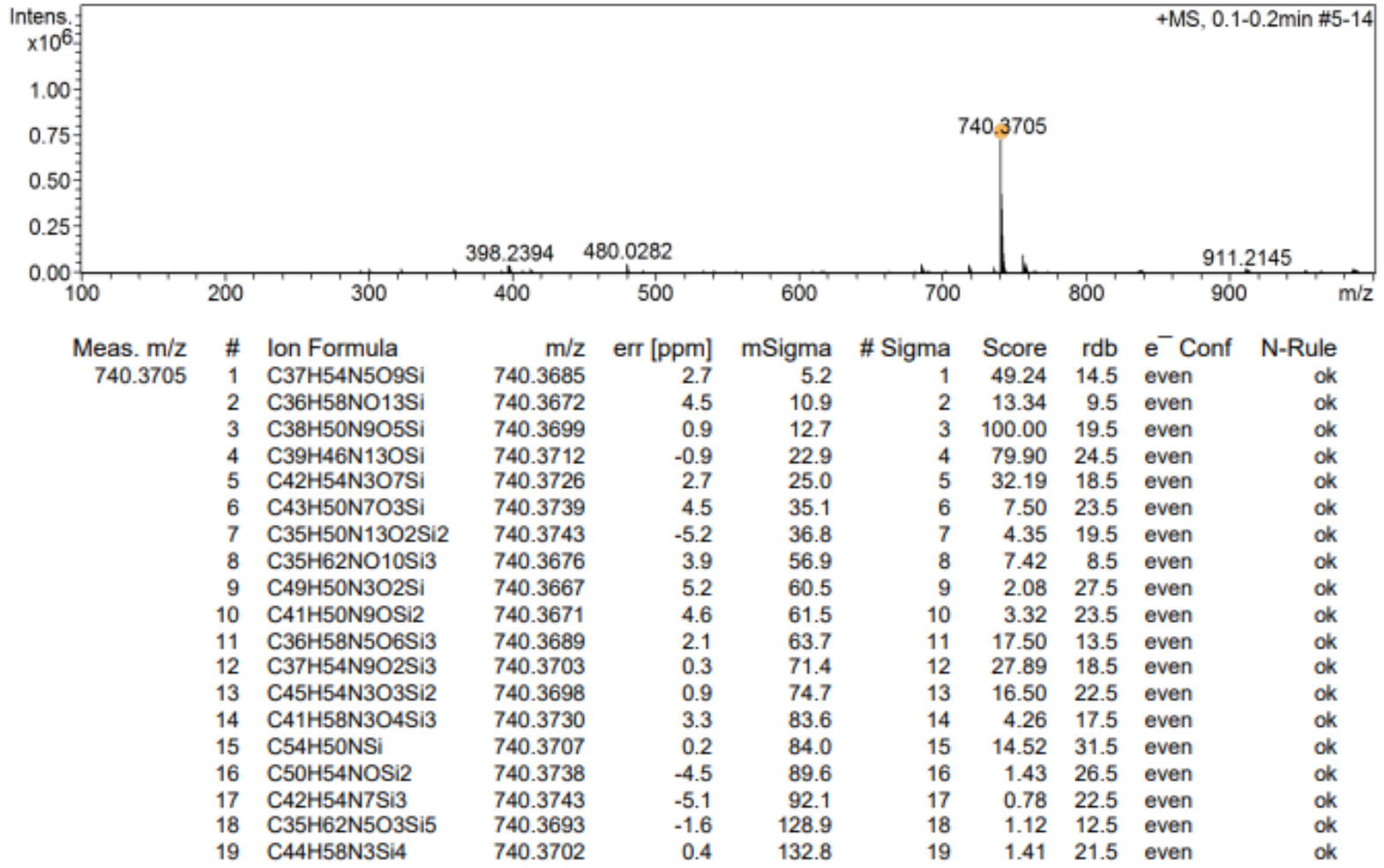

KDY015.d 


\section{Compound Spectrum SmartFormula Report}

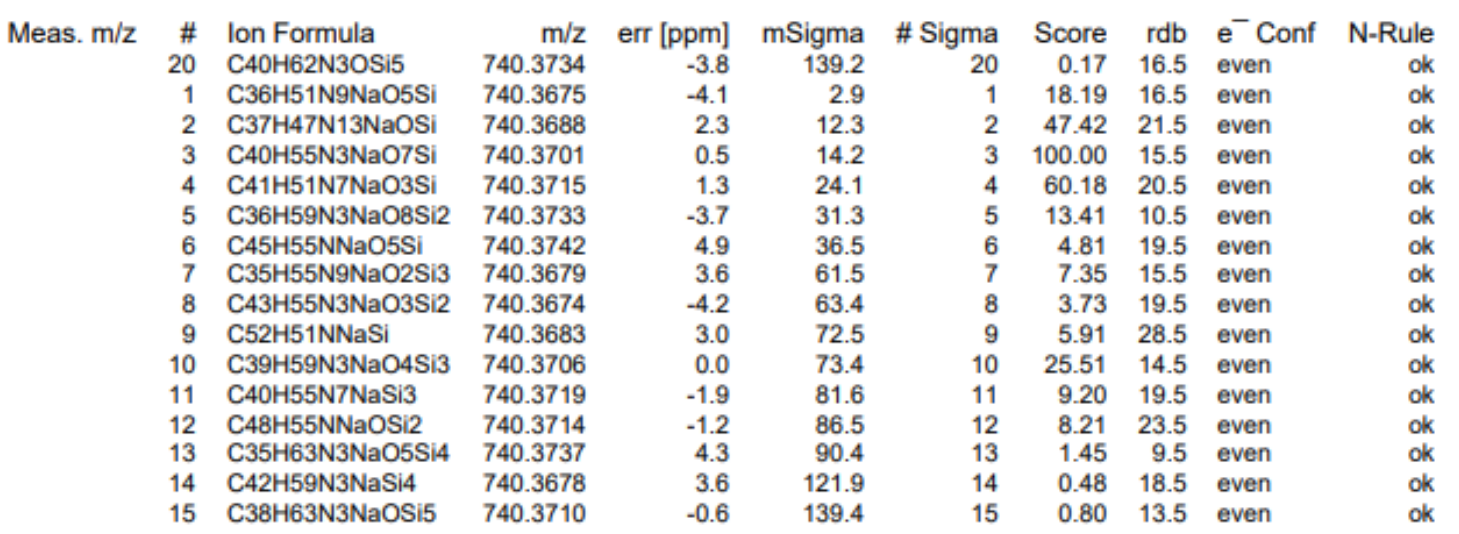

KDY015.d

HR-MS of compound S5 


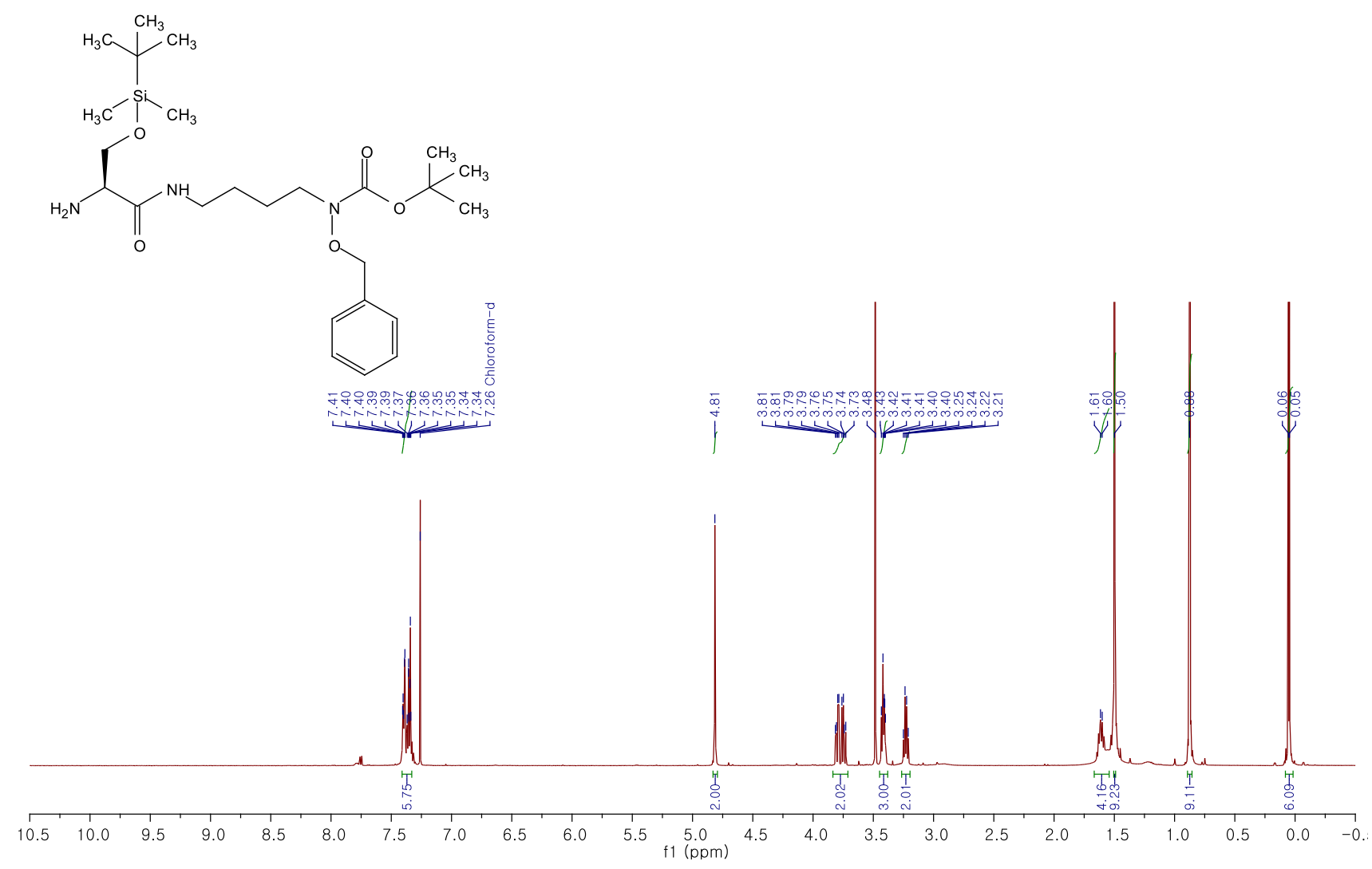

${ }^{1} \mathrm{H}-\mathrm{NMR}$ of compound $\mathbf{1 0 a}\left(500 \mathrm{MHz}, \mathrm{CDCl}_{3}\right)$
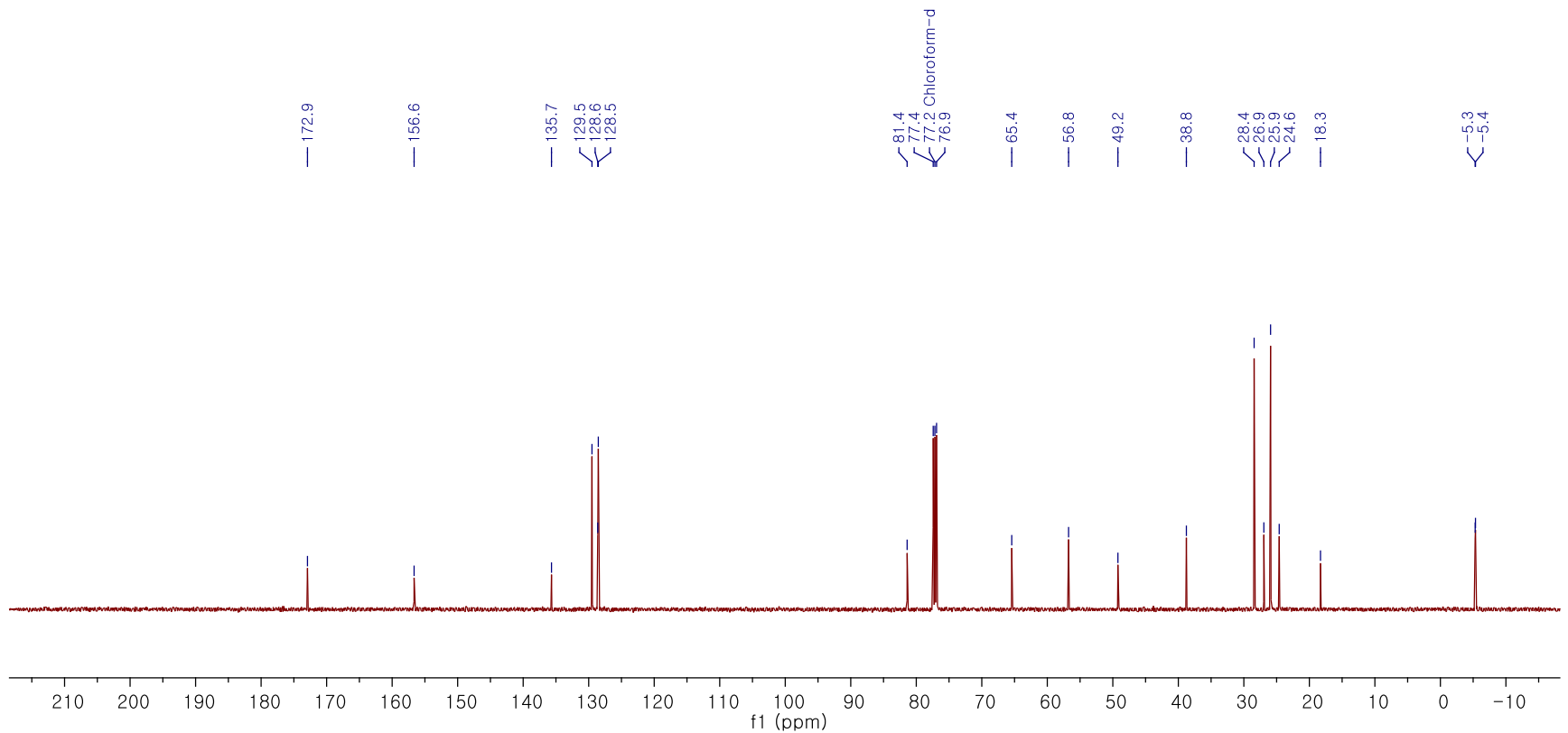

${ }^{13} \mathrm{C}-\mathrm{NMR}$ of compound 10a $\left(125 \mathrm{MHz}, \mathrm{CDCl}_{3}\right)$ 


\section{Compound Spectrum SmartFormula Report}

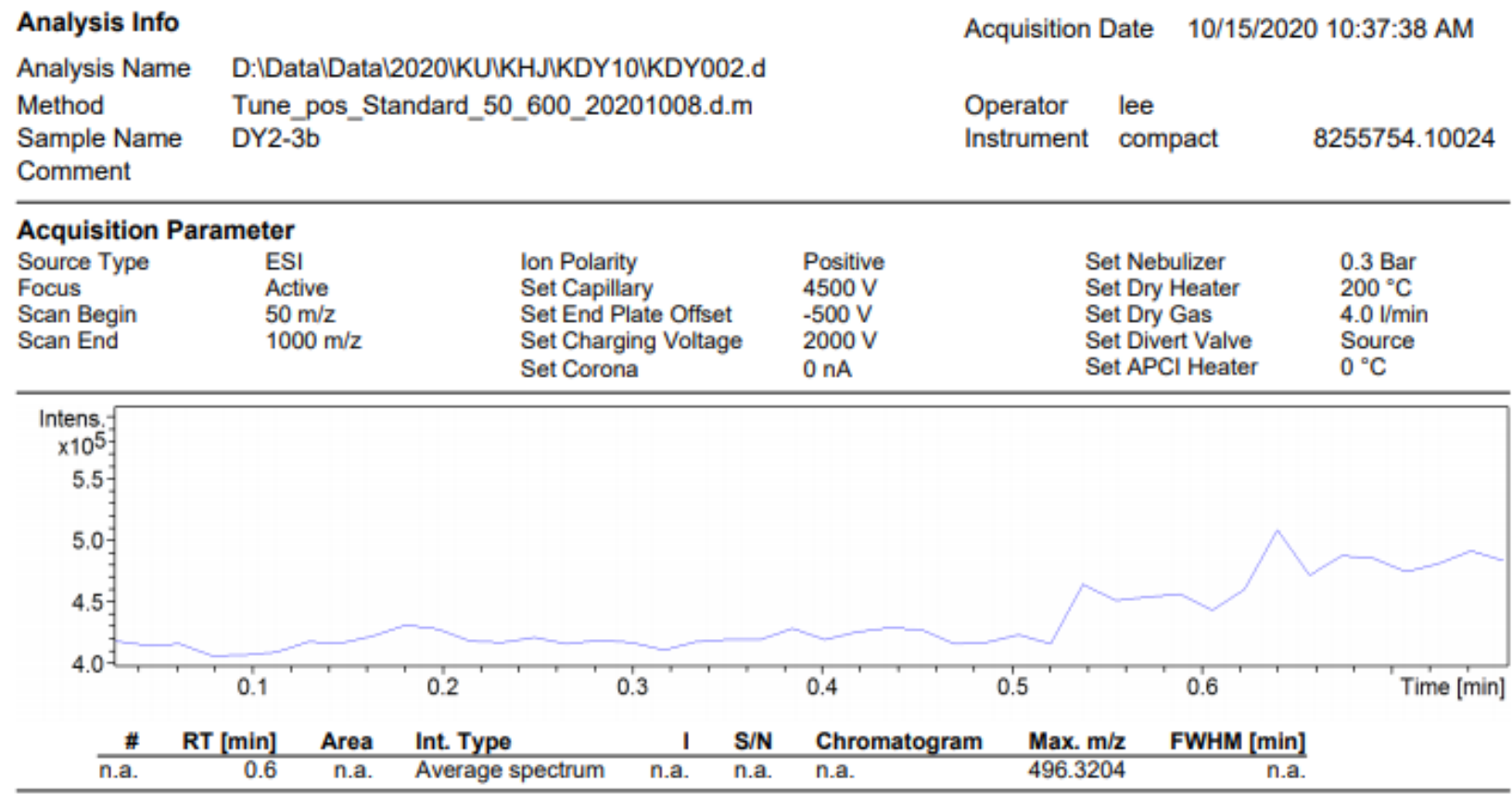

+MS, 0.5-0.7min \#29-42

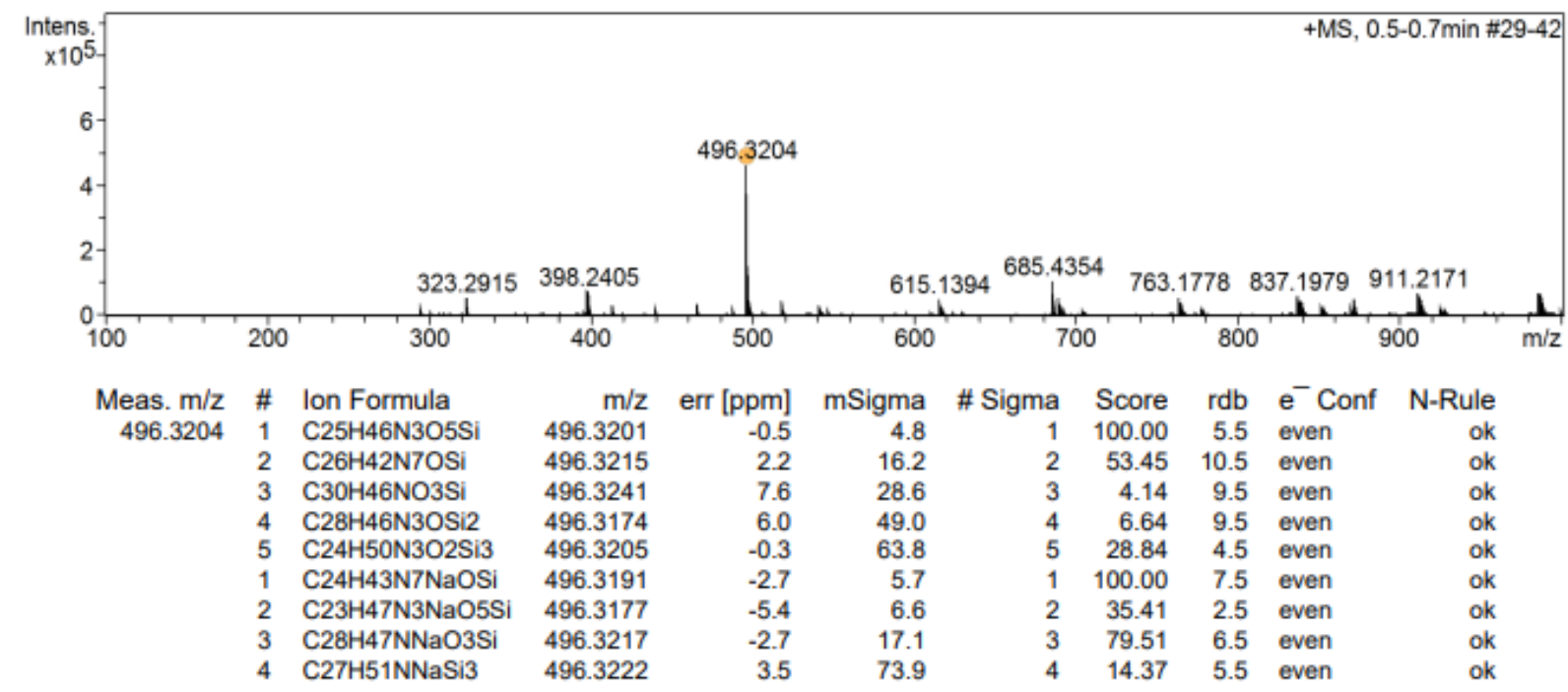

HR-MS of compound 10a 


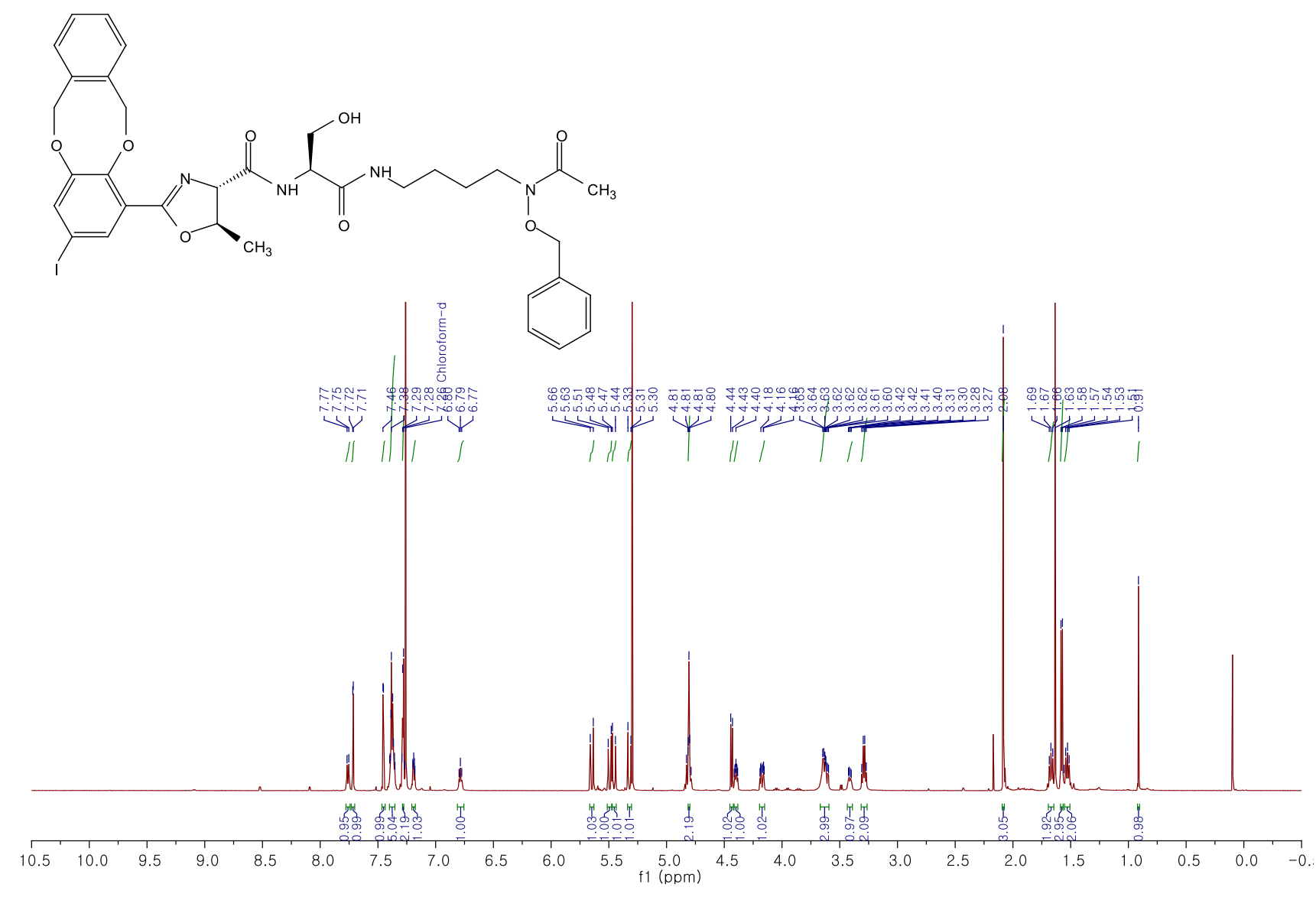

${ }^{1} \mathrm{H}-\mathrm{NMR}$ of compound $\mathbf{1 1 a}\left(500 \mathrm{MHz}, \mathrm{CDCl}_{3}\right)$

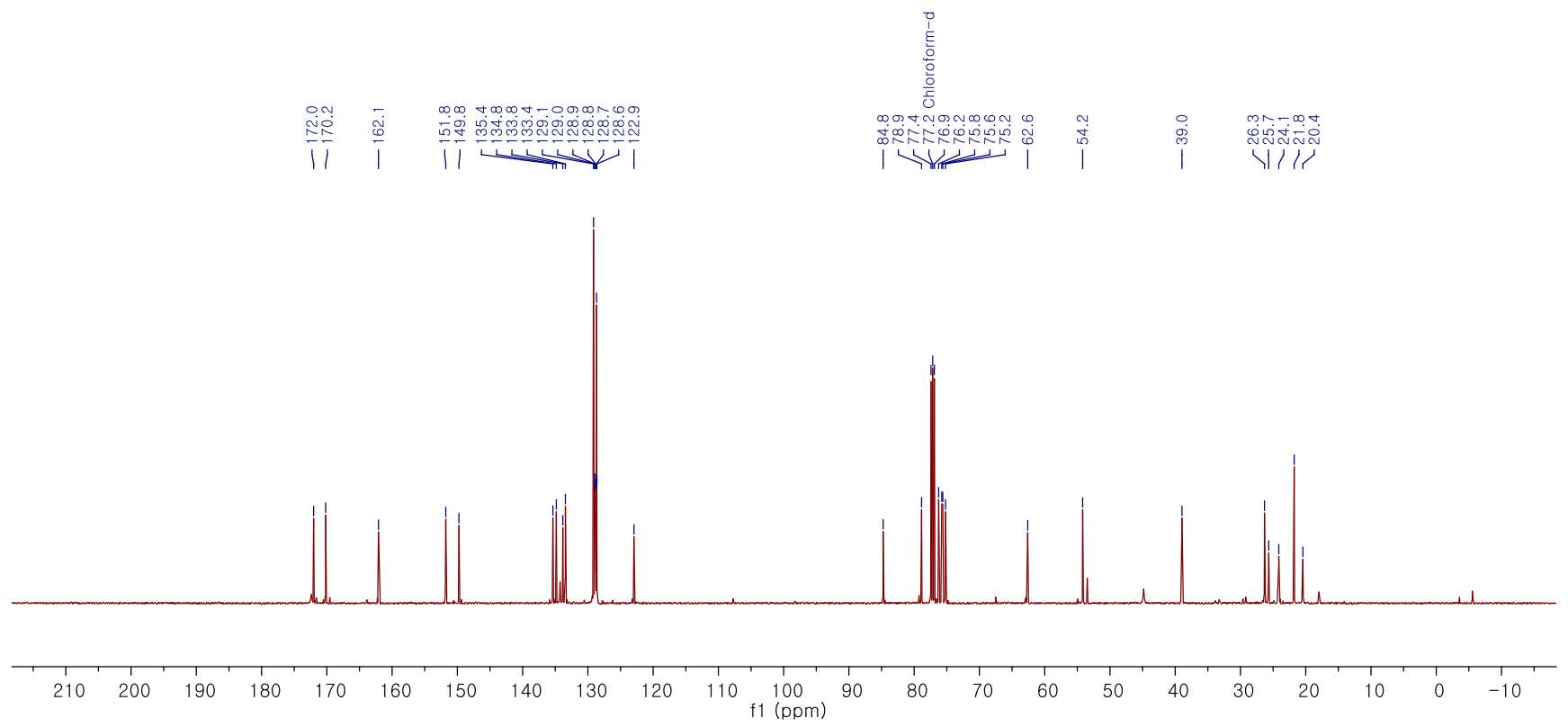

${ }^{13} \mathrm{C}-\mathrm{NMR}$ of compound 11a (125 MHz, $\left.\mathrm{CDCl}_{3}\right)$ 


\section{Compound Spectrum SmartFormula Report}

Analysis Info

Analysis Name

Method

Sample Name

Comment
Acquisition Date $\quad$ 10/15/2020 10:40:26 AM

Operator lee

Instrument compact

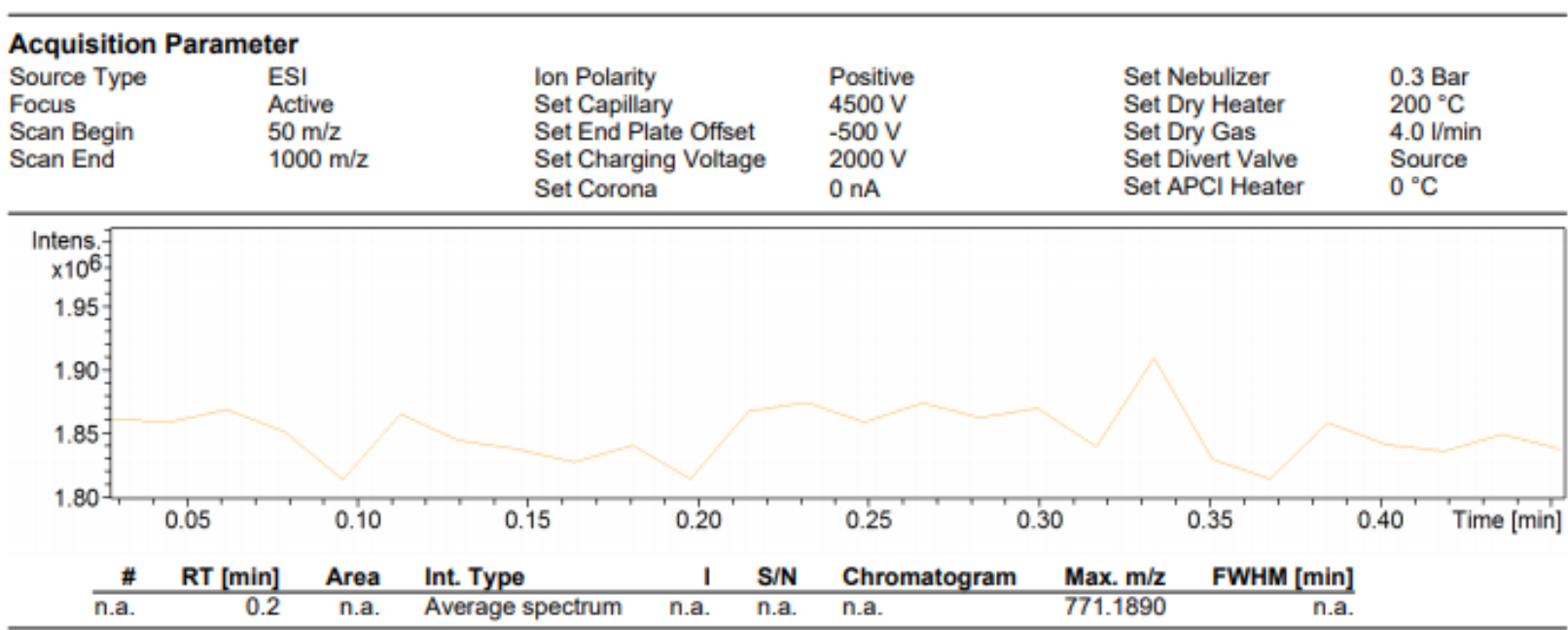

+MS, 0.1-0.3min \#6-17

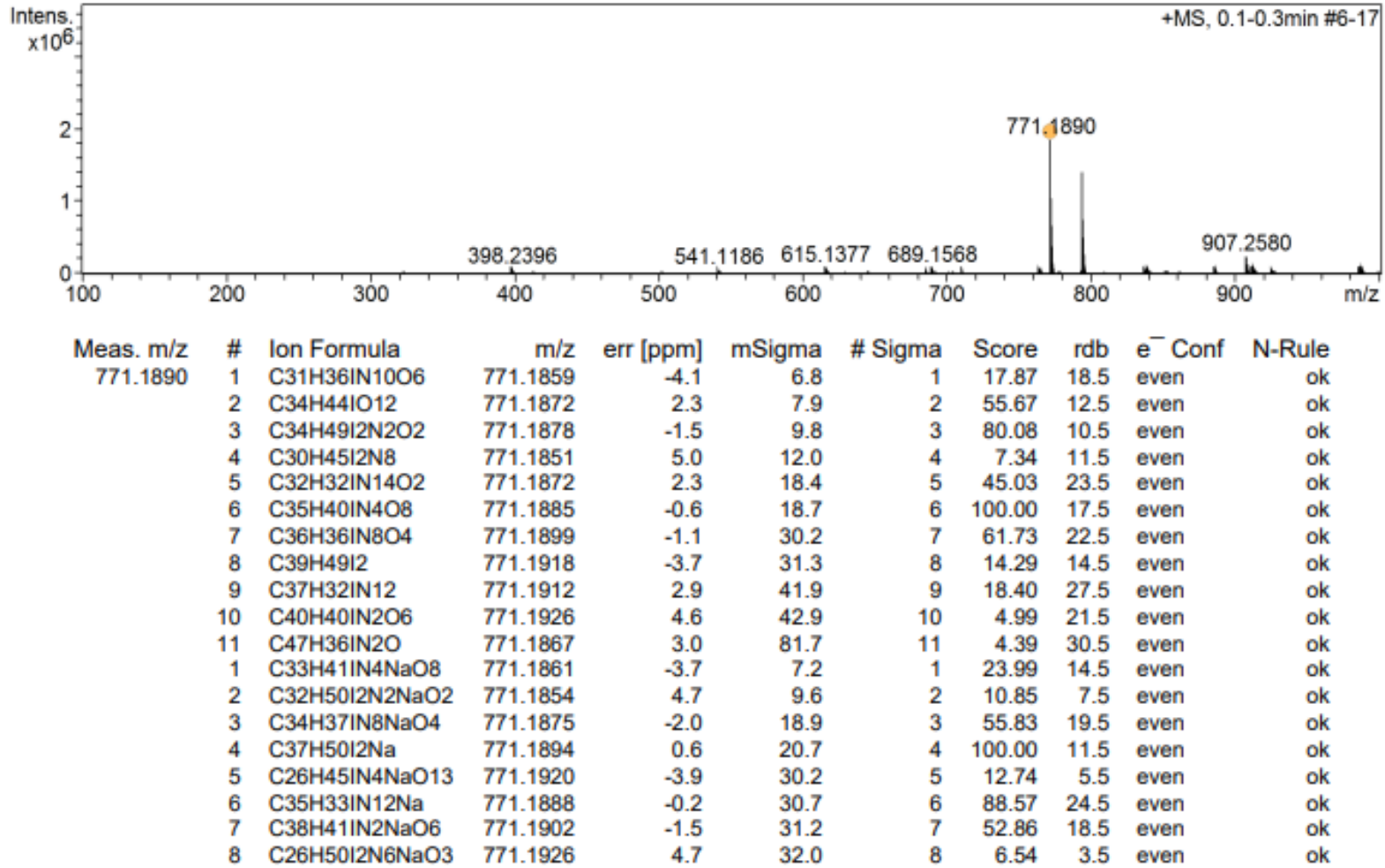

KDY003.d

HR-MS of compound 11a 


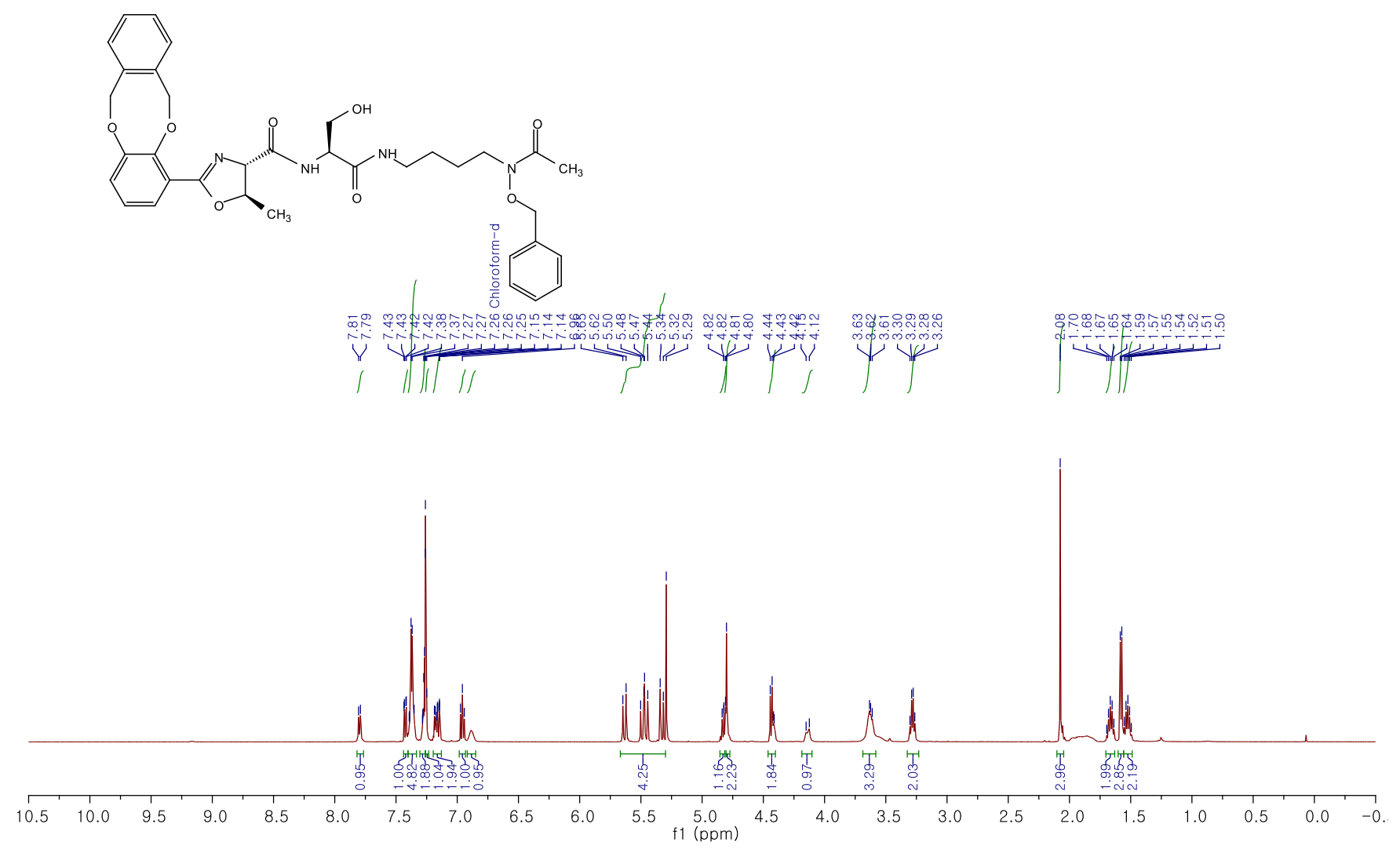

${ }^{1} \mathrm{H}-\mathrm{NMR}$ of compound $\mathbf{1 1 b}\left(500 \mathrm{MHz}, \mathrm{CDCl}_{3}\right.$ ) 


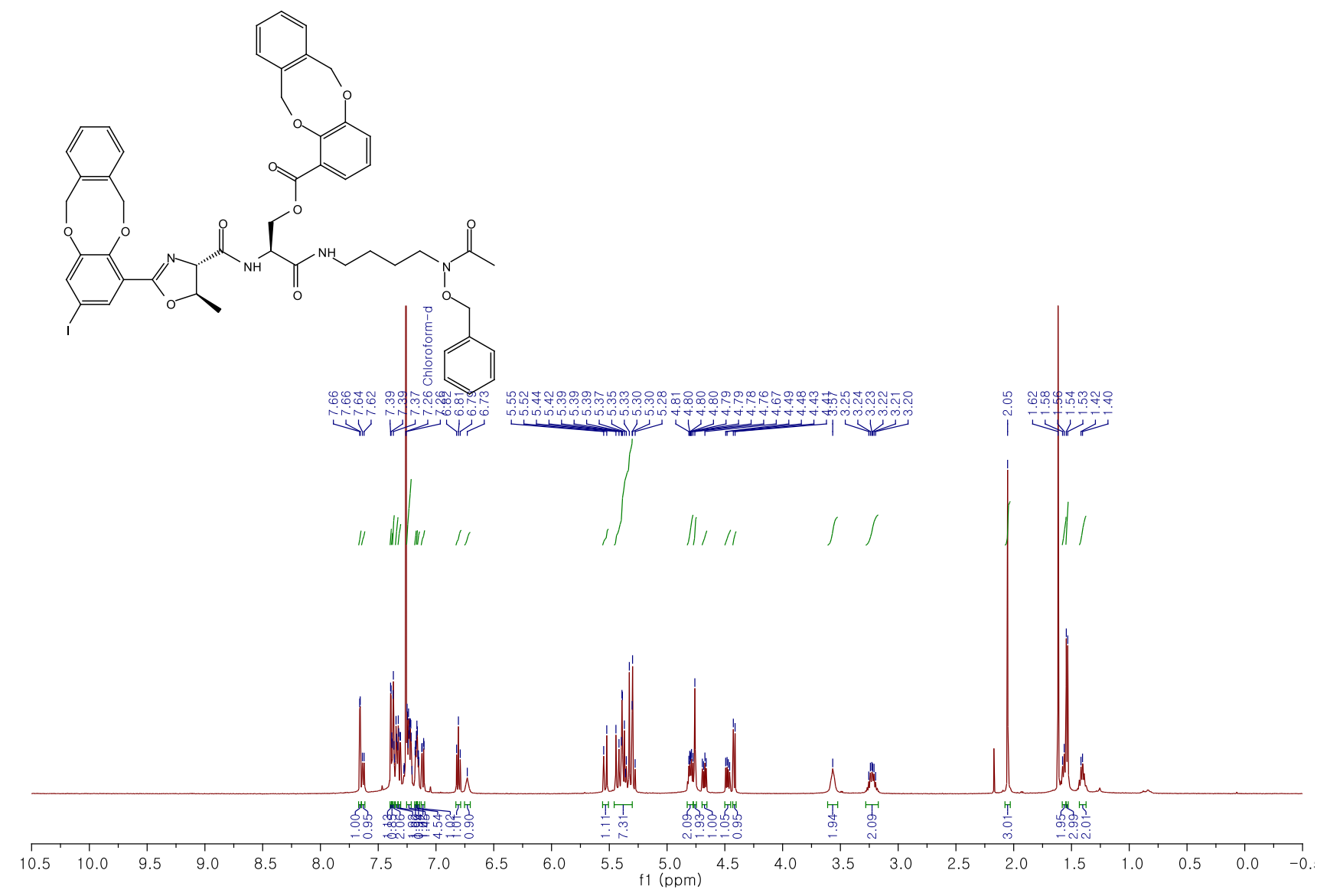

${ }^{1} \mathrm{H}-\mathrm{NMR}$ of compound $\mathbf{1 3 a}\left(500 \mathrm{MHz}, \mathrm{CDCl}_{3}\right.$ )

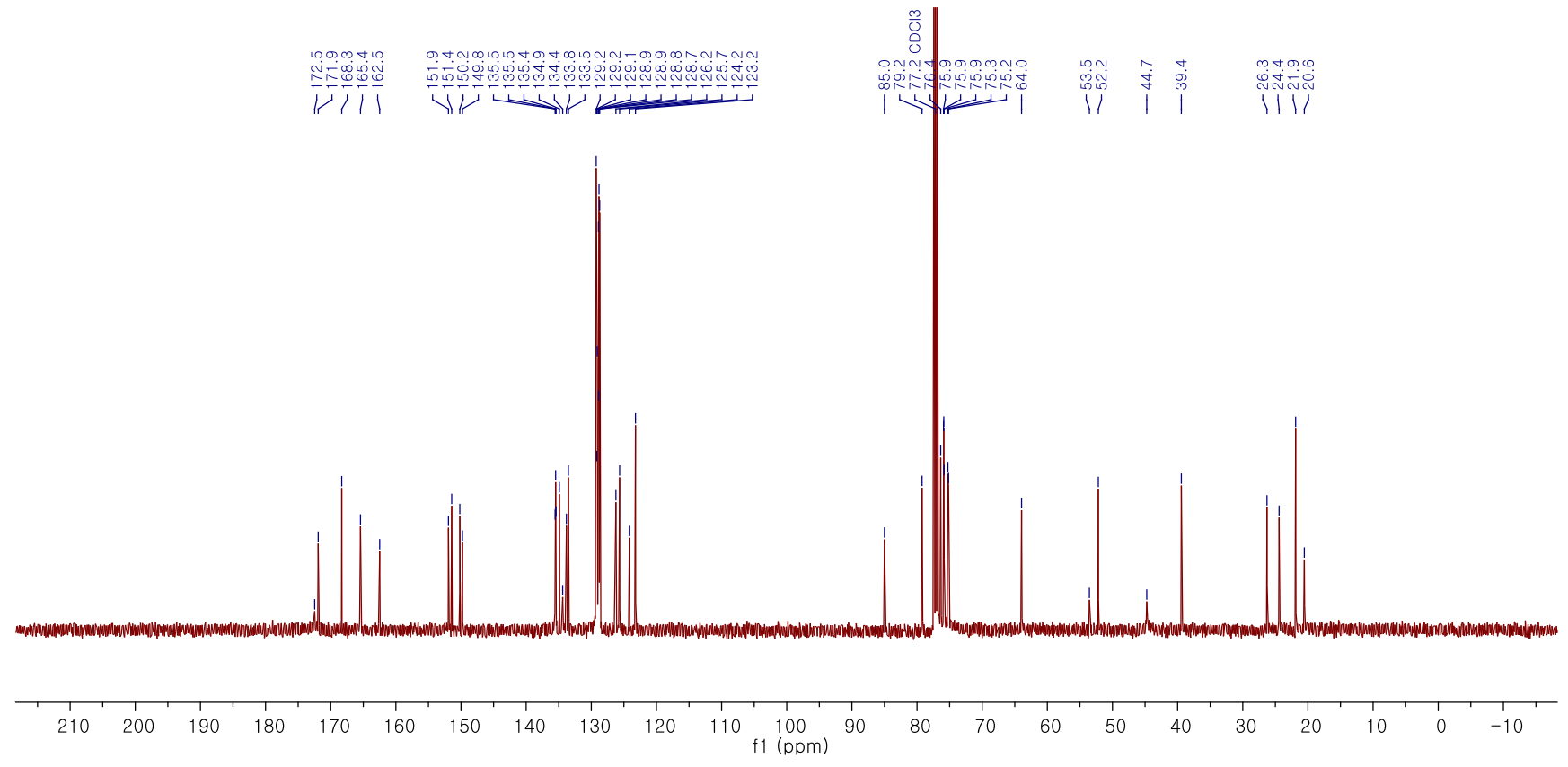

${ }^{13} \mathrm{C}$-NMR of compound 13a (125 MHz, $\left.\mathrm{CDCl}_{3}\right)$ 


\section{Compound Spectrum SmartFormula Report}

\section{Analysis Info}

Analysis Name

Method

Sample Name

Comment
Acquisition Date $\quad$ 10/16/2020 3:19:47 PM

D:IDatalDatal2020UKUIKHJKDY10IKDY008-1.d

Tune_pos_Standard_50_600_20201008.d.m Operator lee

DY8-6b Instrument compact

8255754.10024

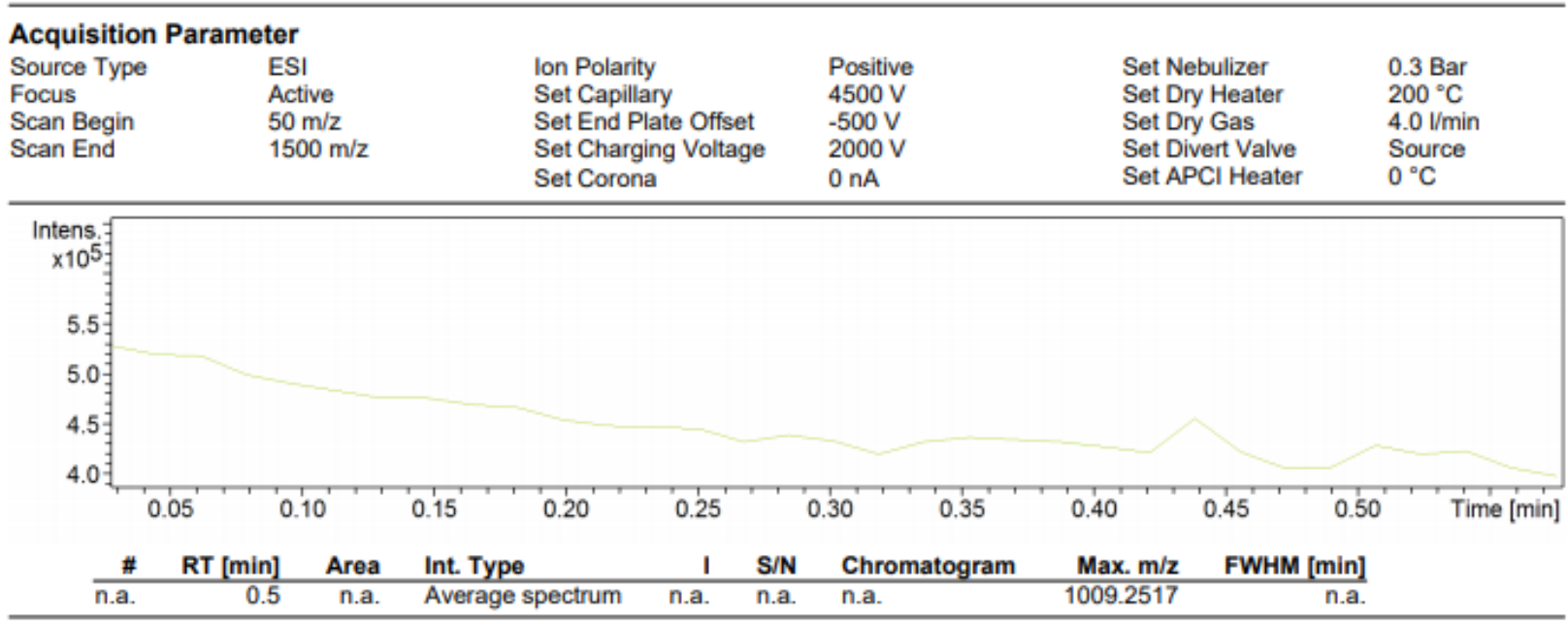

+MS, 0.4-0.6min \#22-32

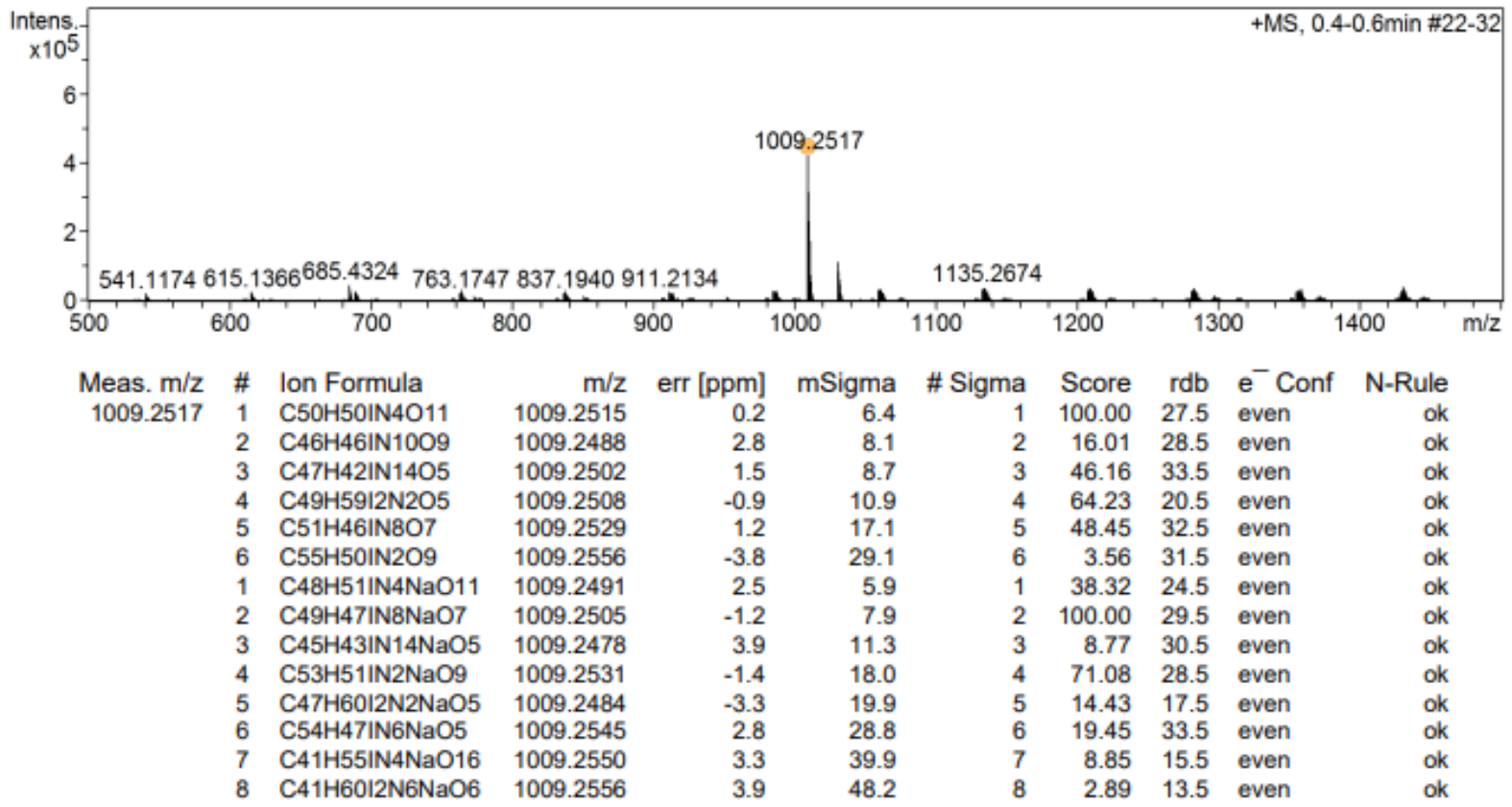

KDY008-1.d

HR-MS of compound 13a 


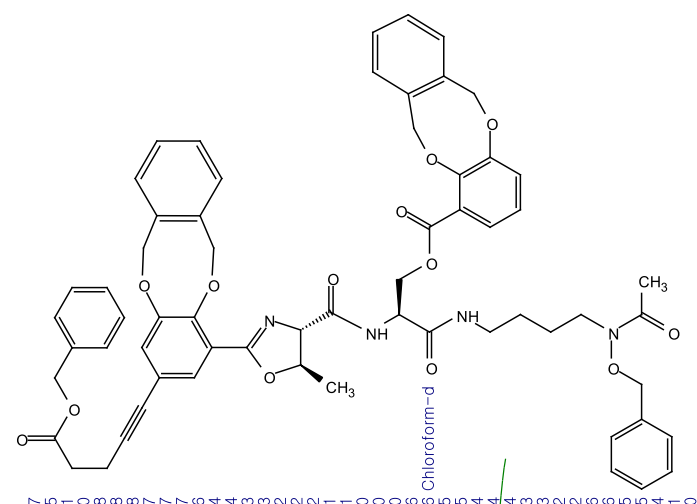

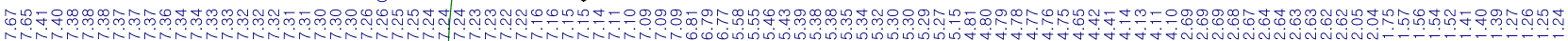
作
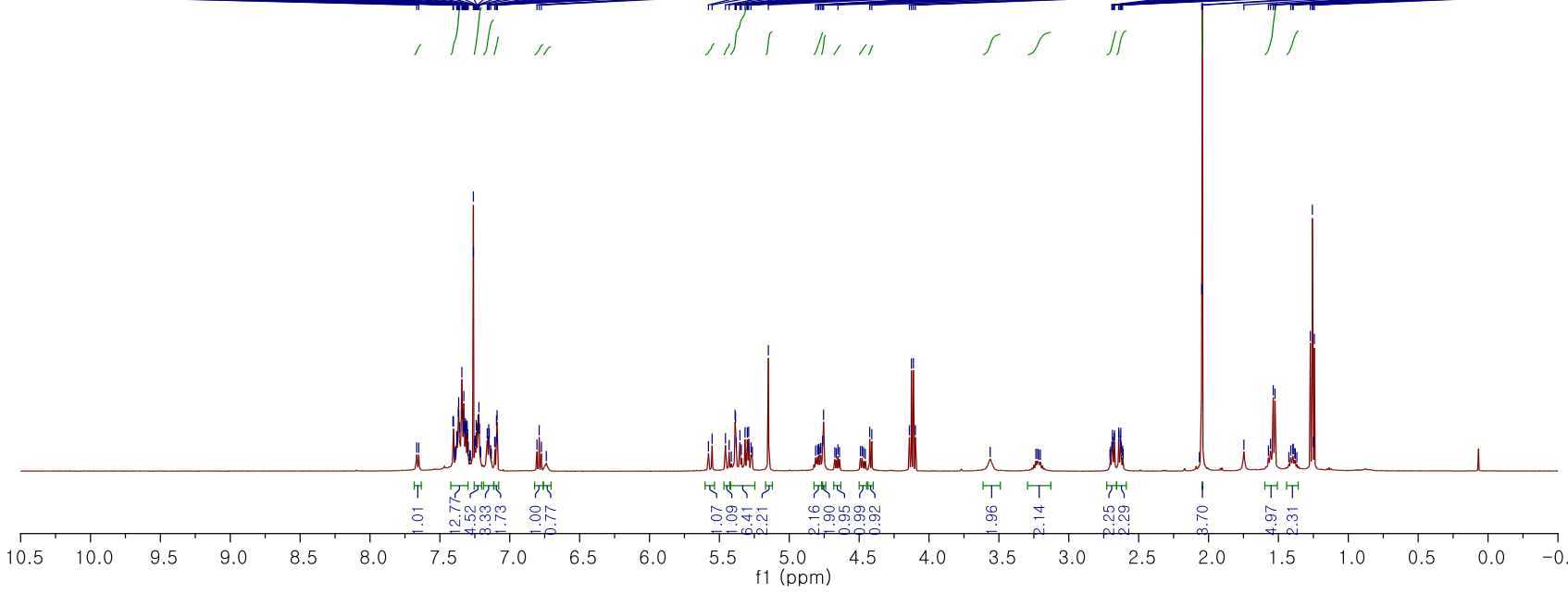

${ }^{1} \mathrm{H}-\mathrm{NMR}$ of compound $\mathbf{1 6 a}\left(500 \mathrm{MHz}, \mathrm{CDCl}_{3}\right)$
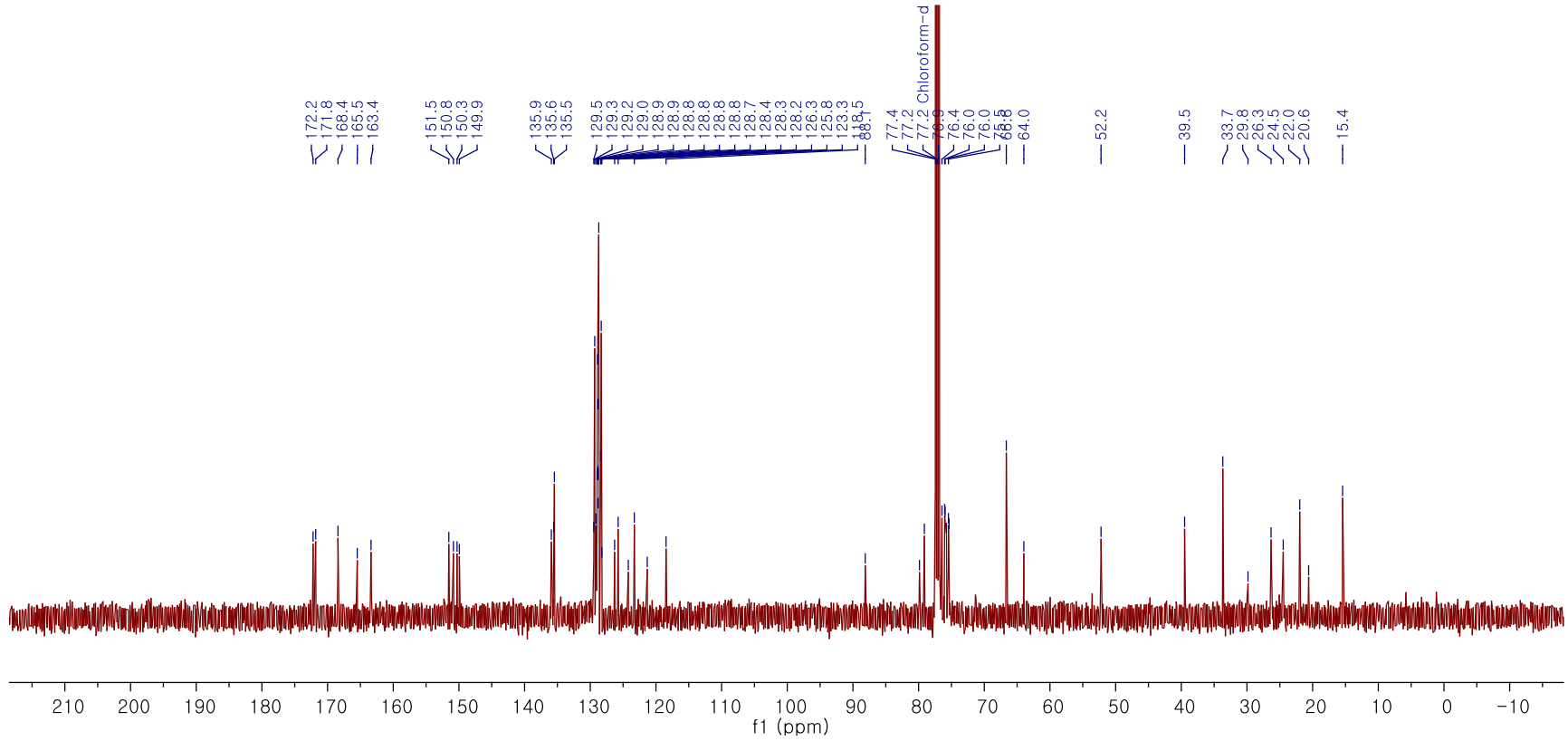

${ }^{13} \mathrm{C}-\mathrm{NMR}$ of compound $\mathbf{1 6 a}\left(125 \mathrm{MHz}, \mathrm{CDCl}_{3}\right)$ 


\section{Compound Spectrum SmartFormula Report}

Analysis Info

Analysis Name

Method

Sample Name

Comment
Acquisition Date $\quad$ 4/26/2021 7:53:19 PM

D:IDatalMCCBIKDY 210426।210426-KDY-B13' intenal calibration.d

NaFormate_50_1600_MS method_POS.m

B13'

Operator

@DE

8255754.20060

\begin{tabular}{|c|c|c|c|c|c|}
\hline \multicolumn{6}{|c|}{ Acquisition Parameter } \\
\hline $\begin{array}{l}\text { Source Type } \\
\text { Focus } \\
\text { Scan Begin } \\
\text { Scan End }\end{array}$ & $\begin{array}{l}\text { ESI } \\
\text { Active } \\
50 \mathrm{~m} / \mathrm{z} \\
1600 \mathrm{~m} / \mathrm{z}\end{array}$ & $\begin{array}{l}\text { Ion Polarity } \\
\text { Set Capillary } \\
\text { Set End Plate Offset } \\
\text { Set Charging Voltage } \\
\text { Set Corona }\end{array}$ & $\begin{array}{l}\text { Positive } \\
4500 \mathrm{~V} \\
-500 \mathrm{~V} \\
2000 \mathrm{~V} \\
0 \mathrm{nA}\end{array}$ & $\begin{array}{l}\text { Set Nebulizer } \\
\text { Set Dry Heater } \\
\text { Set Dry Gas } \\
\text { Set Divert Valve } \\
\text { Set APCI Heater }\end{array}$ & $\begin{array}{l}0.4 \mathrm{Bar} \\
200^{\circ} \mathrm{C} \\
4.0 \mathrm{~V} / \mathrm{min} \\
\text { Source } \\
0^{\circ} \mathrm{C}\end{array}$ \\
\hline \multicolumn{6}{|l|}{$\begin{array}{r}\text { Intens. } \\
\times 10^{6}=\end{array}$} \\
\hline \multicolumn{6}{|l|}{ 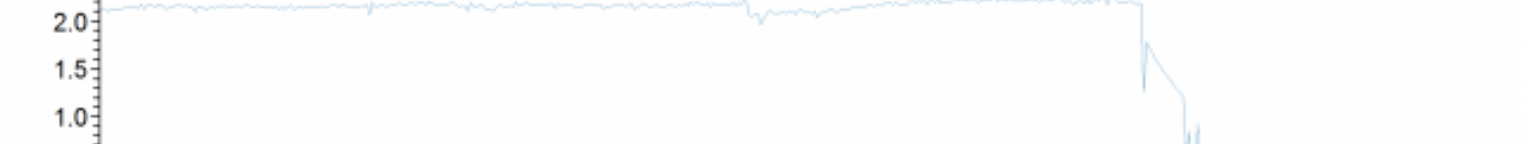 } \\
\hline & 1 & 3 & 5 & 7 & Tin \\
\hline
\end{tabular}

$+\mathrm{MS}, 7.0 \mathrm{~min} \# 412$

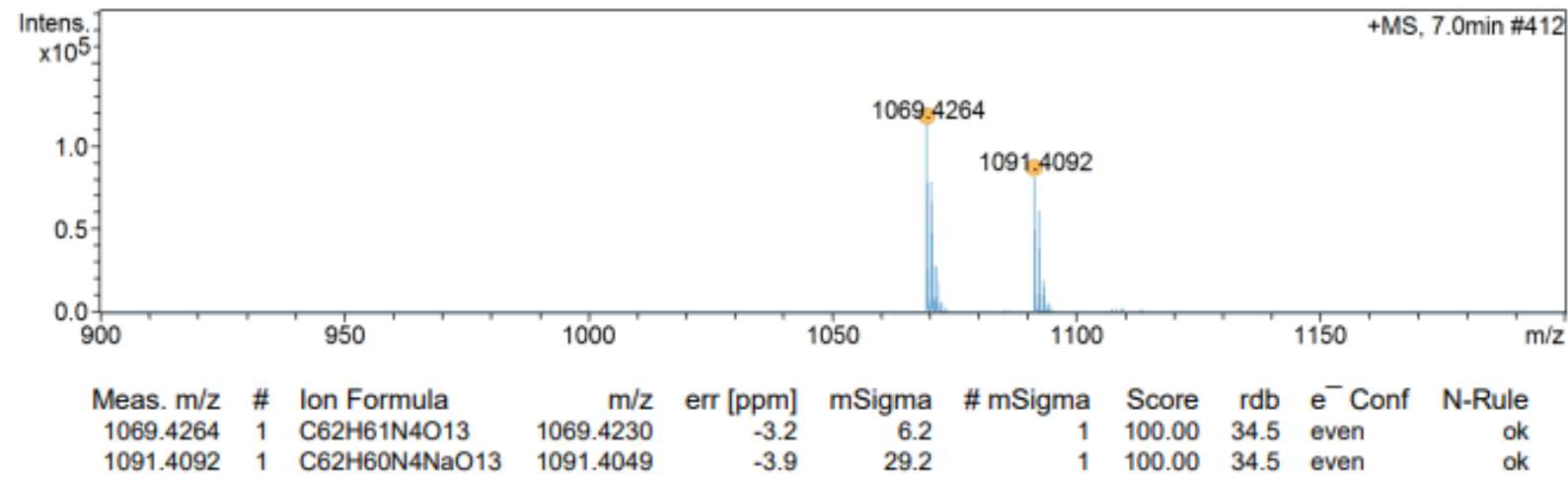

HR-MS of compound 16a 


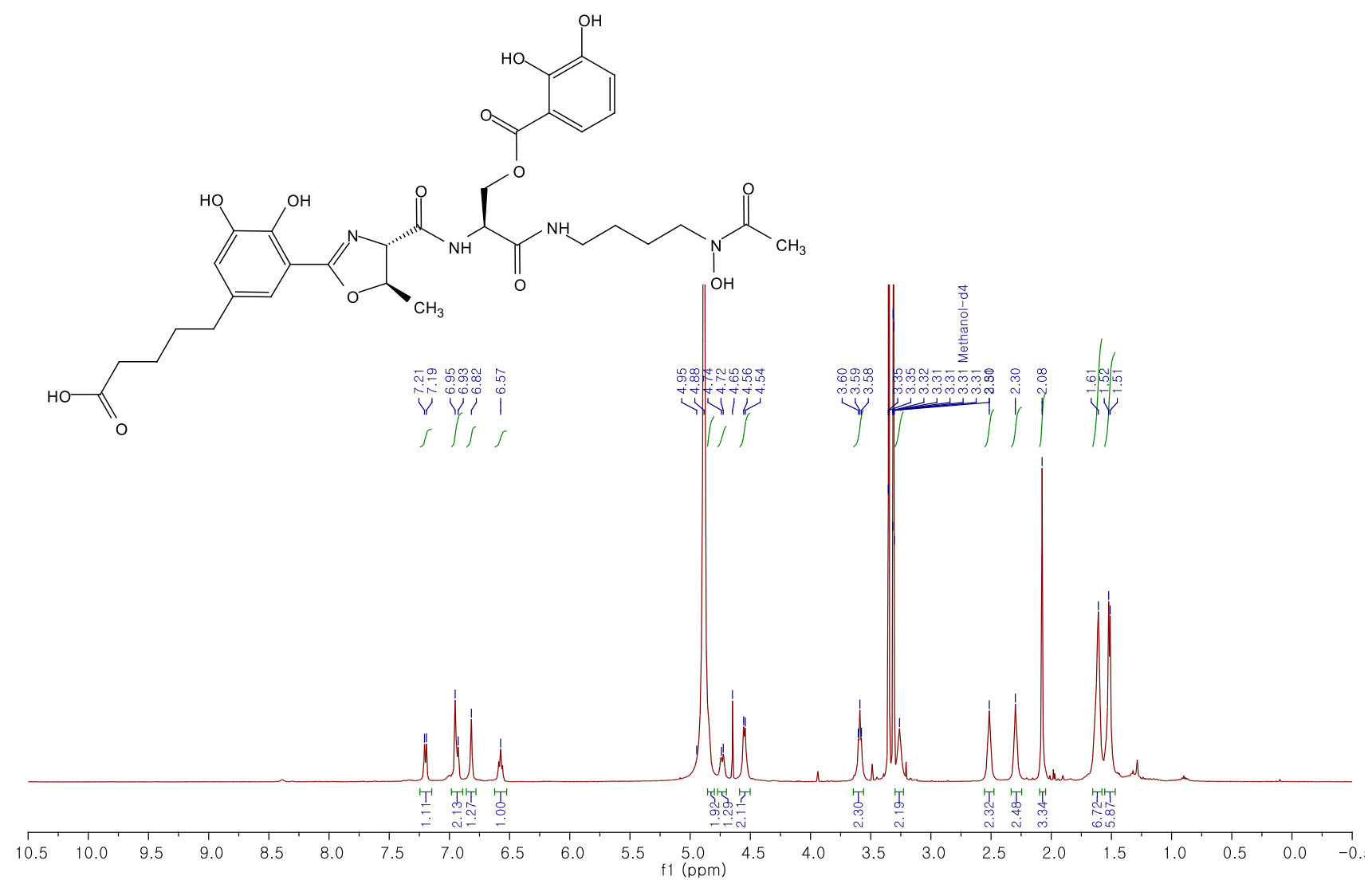

${ }^{1} \mathrm{H}-\mathrm{NMR}$ of compound $\mathbf{S 6}\left(500 \mathrm{MHz}, \mathrm{CD}_{3} \mathrm{OD}\right)$

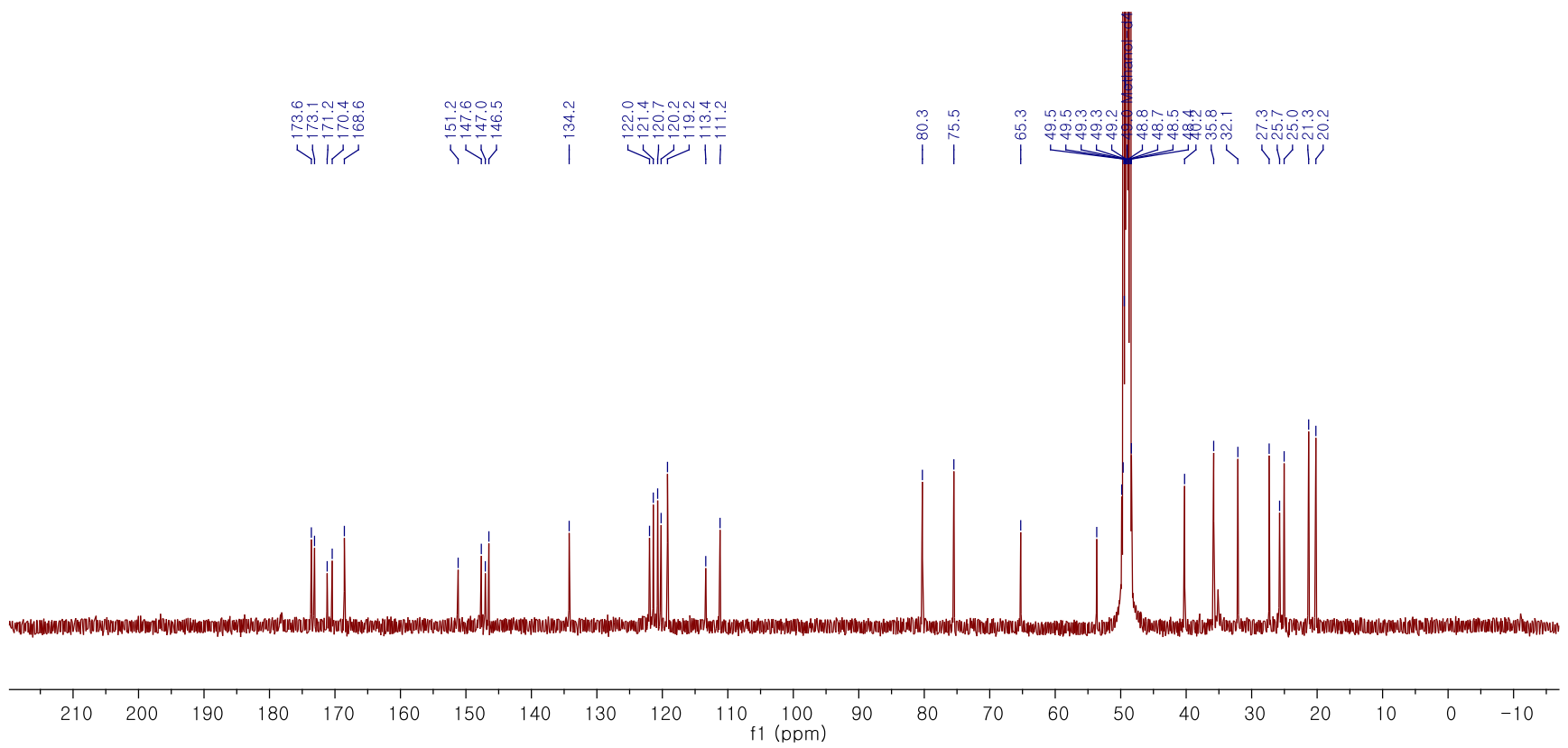

${ }^{13} \mathrm{C}$-NMR of compound $\mathbf{S 6}$ (125 MHz, $\left.\mathrm{CD}_{3} \mathrm{OD}\right)$ 


\section{Compound Spectrum SmartFormula Report}

\section{Analysis Info}

Analysis Name

Method

Sample Name

Comment
Acquisition Date 4/26/2021 9:55:35 PM

D:IDatalMCCBIKDY2104261210426-KDY-B14'.d

NaFormate_50_1600_MS method_POS.m

B14'
Operator BDAL@DE

Instrument compact

8255754.20060

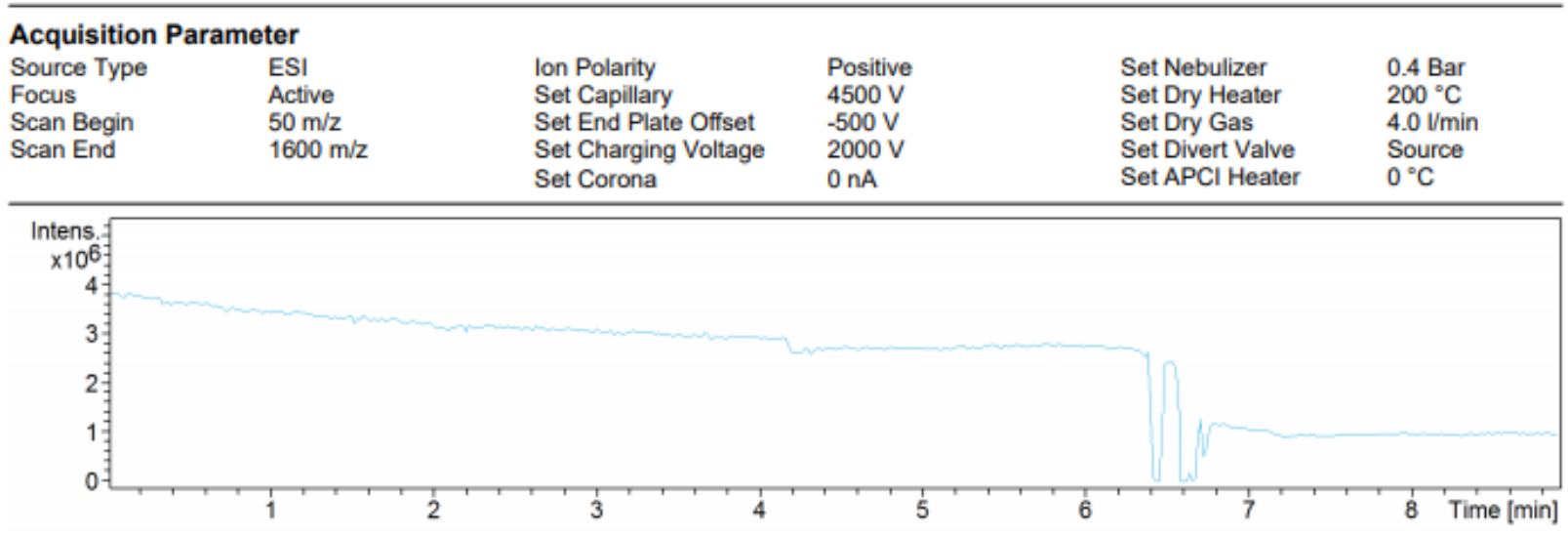

+MS, 6.9-6.9min \#404-406

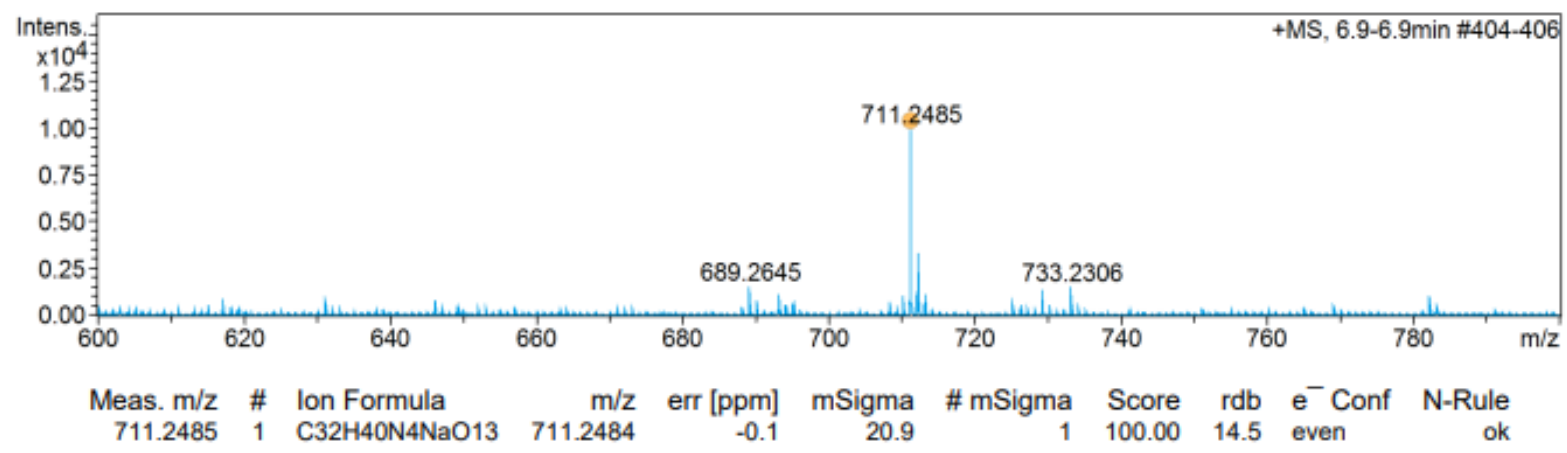

HR-MS of compound S6 


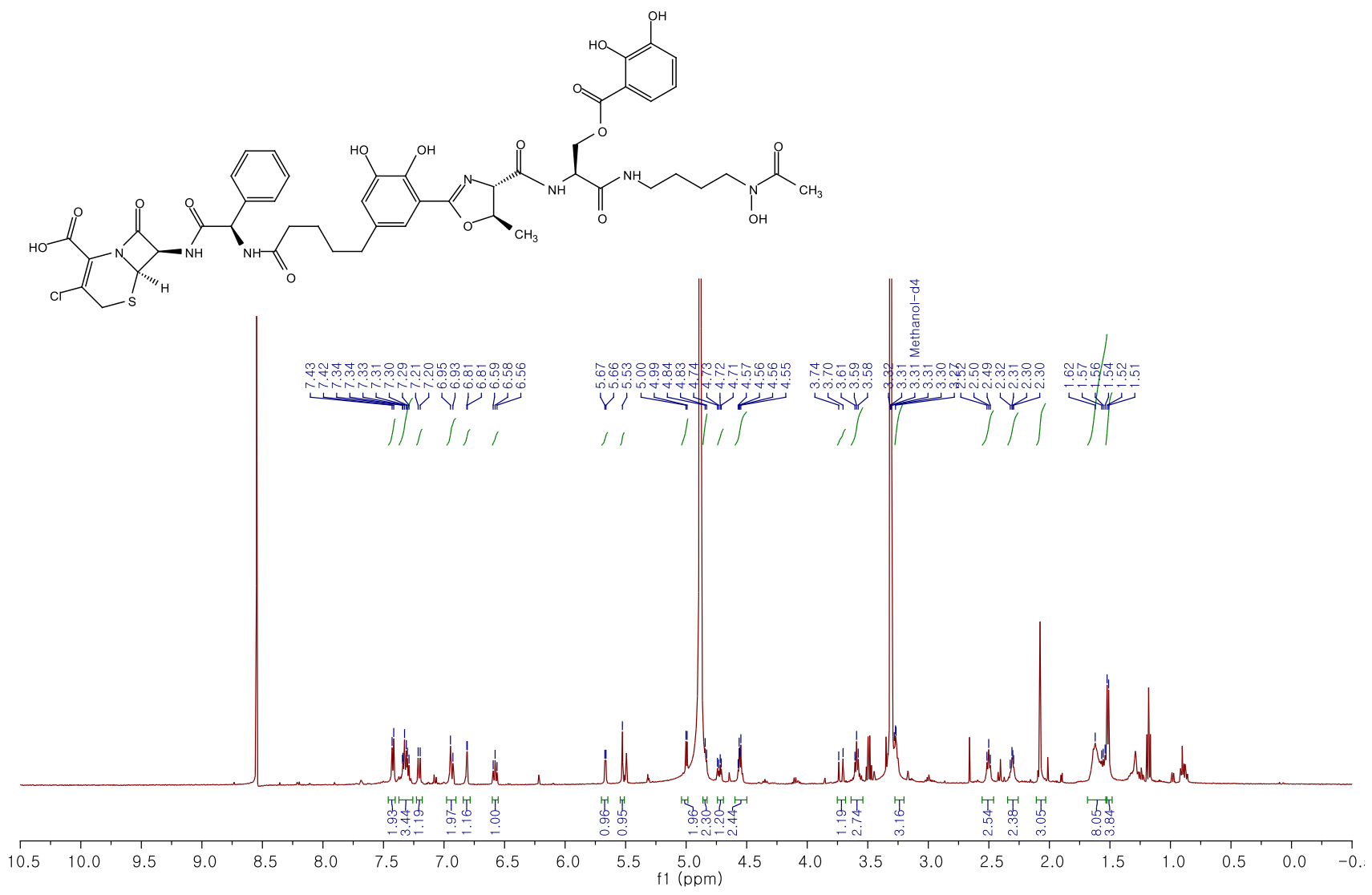

${ }^{1} \mathrm{H}-\mathrm{NMR}$ of compound 18a (500 MHz, $\left.\mathrm{CD}_{3} \mathrm{OD}\right)$

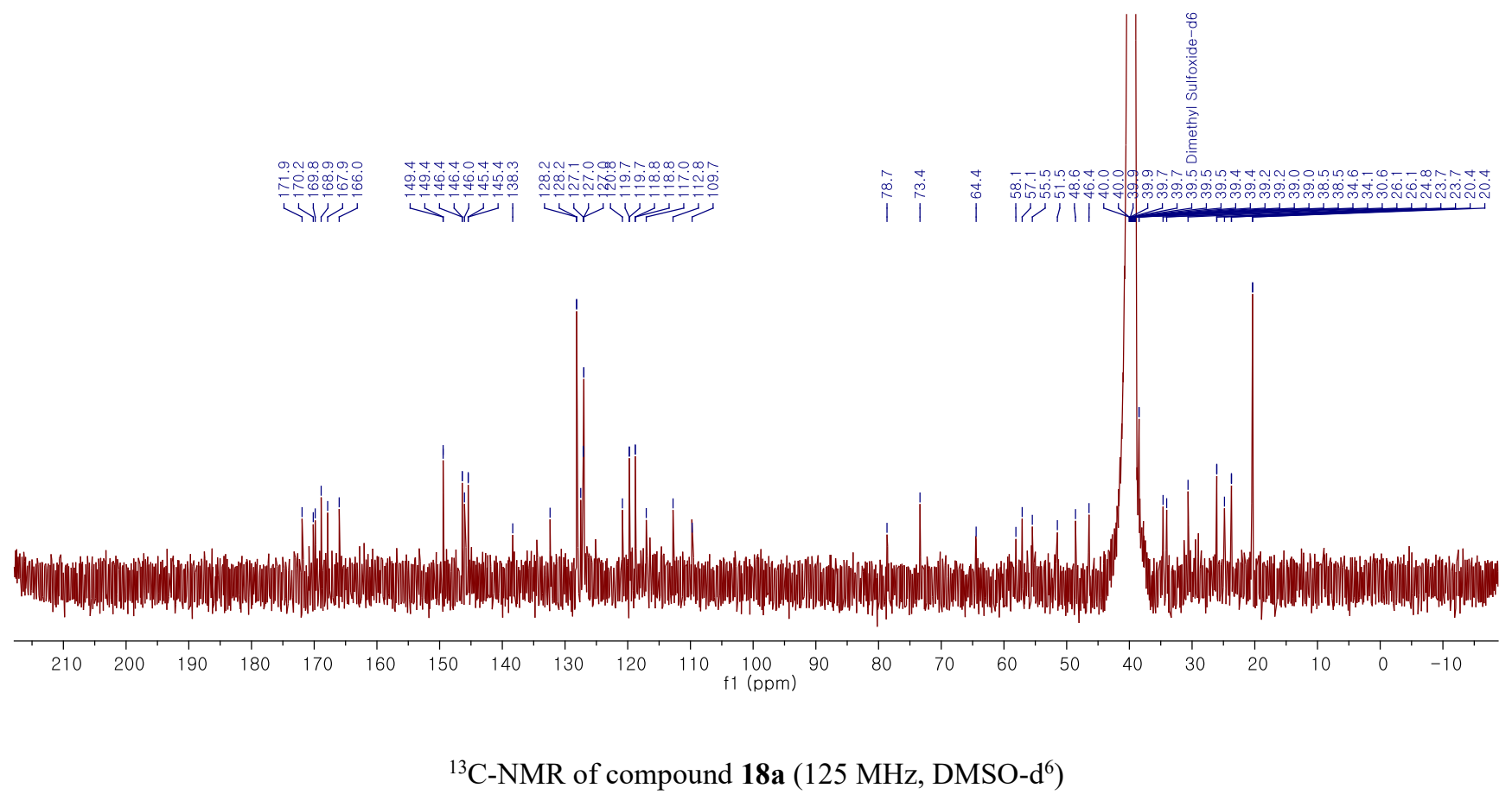




\section{Compound Spectrum SmartFormula Report}

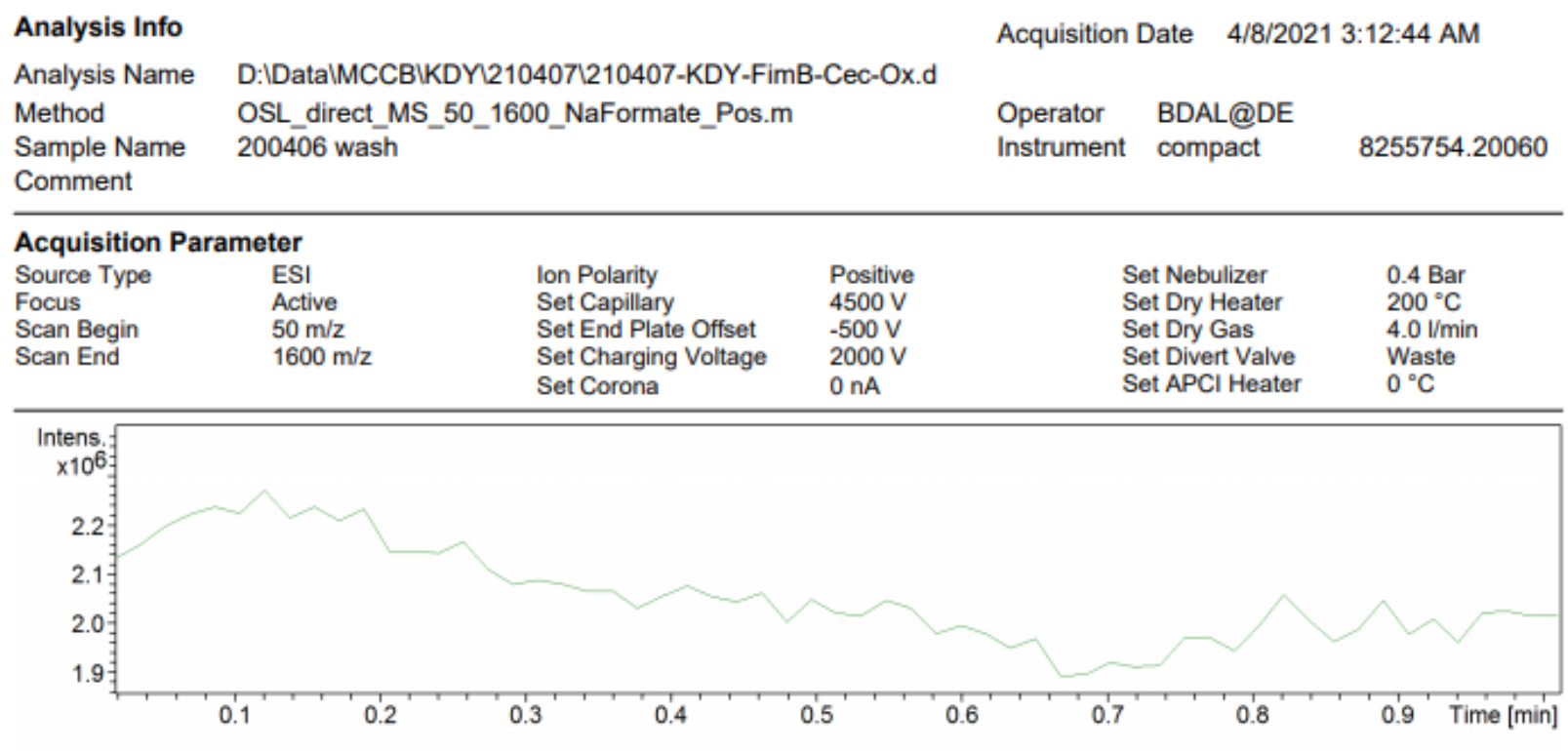

+MS, 0.6-1.0min \#36-57

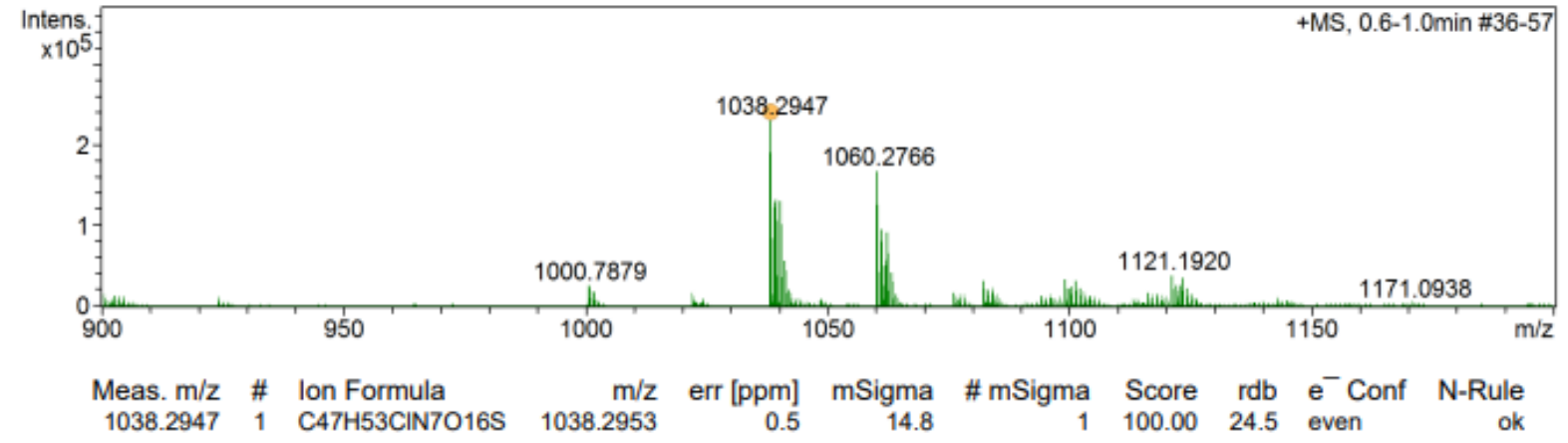

HR-MS of compound 18a 


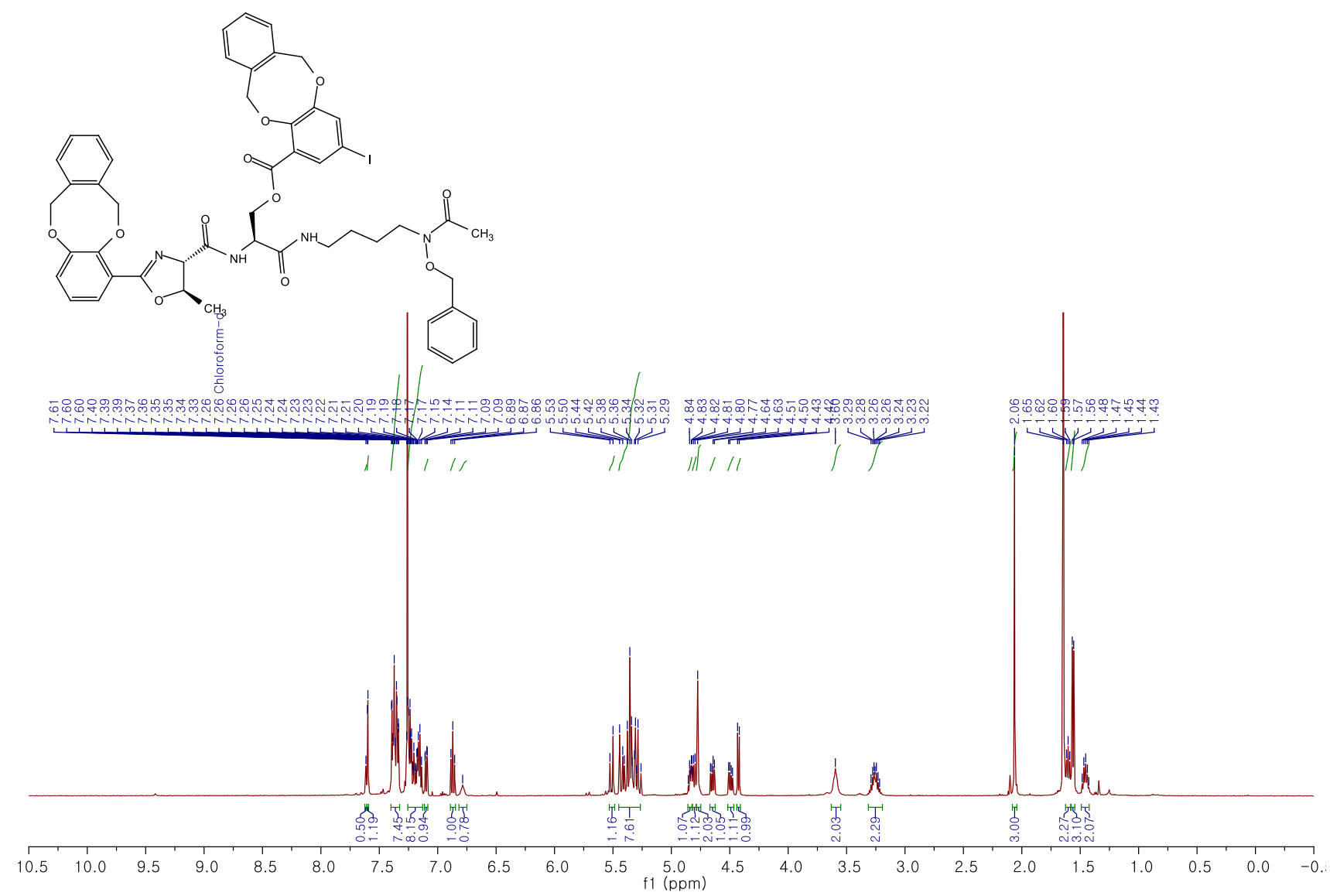

${ }^{1} \mathrm{H}-\mathrm{NMR}$ of compound $\mathbf{1 3 b}\left(500 \mathrm{MHz}, \mathrm{CDCl}_{3}\right)$

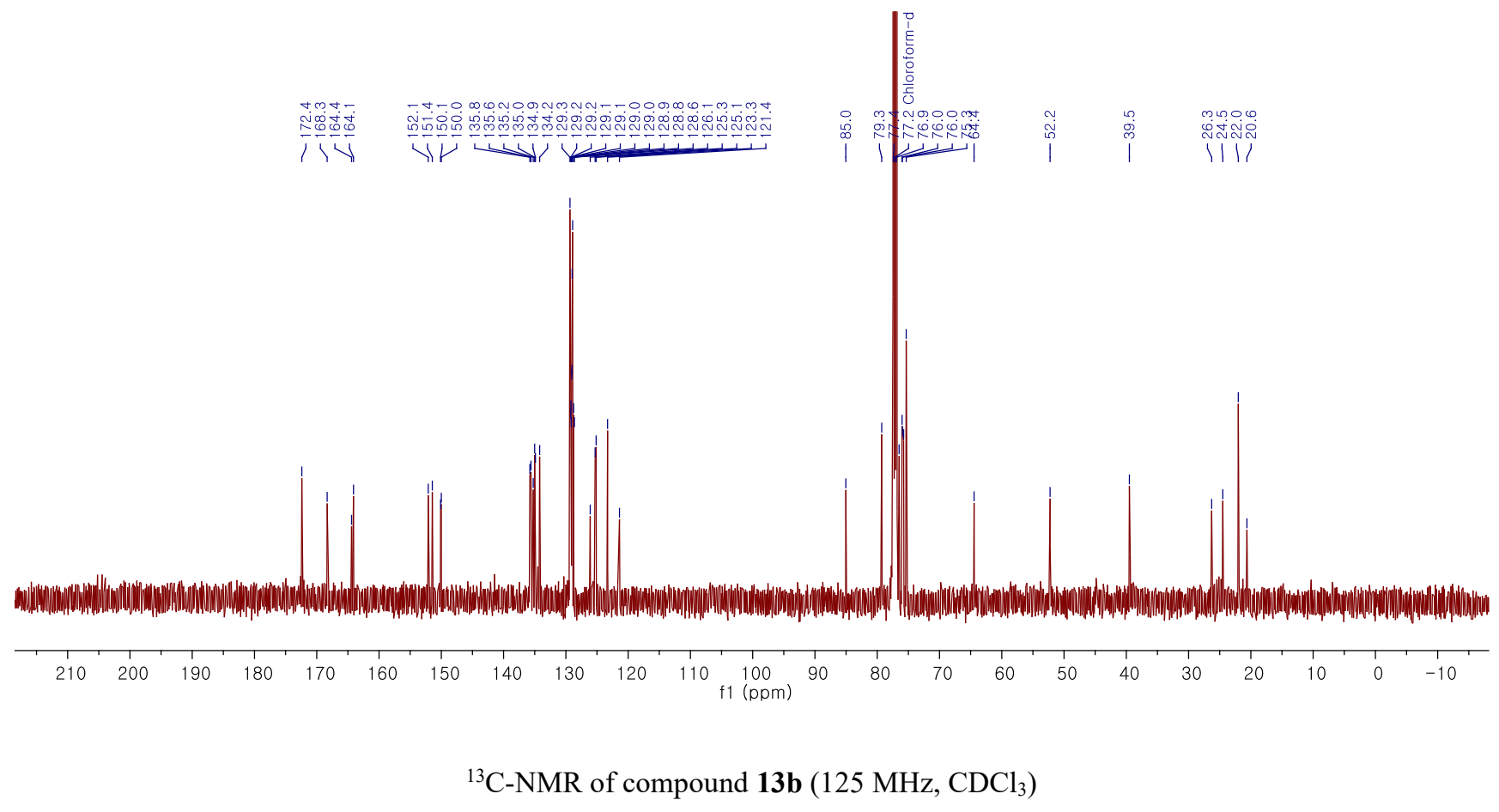




\section{Compound Spectrum SmartFormula Report}

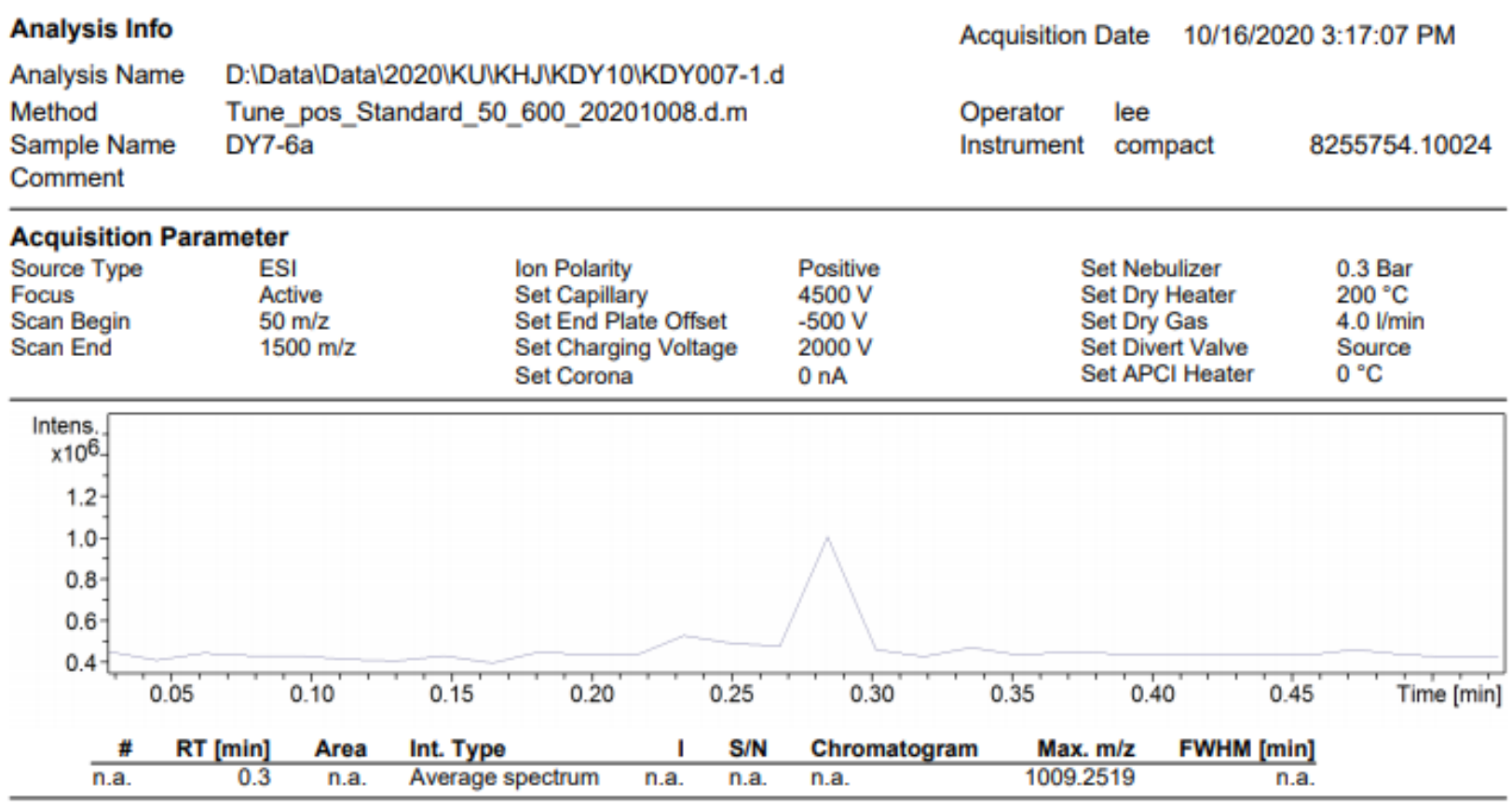

$+\mathrm{MS}, 0.2-0.4 \mathrm{~min} \# 9-24$

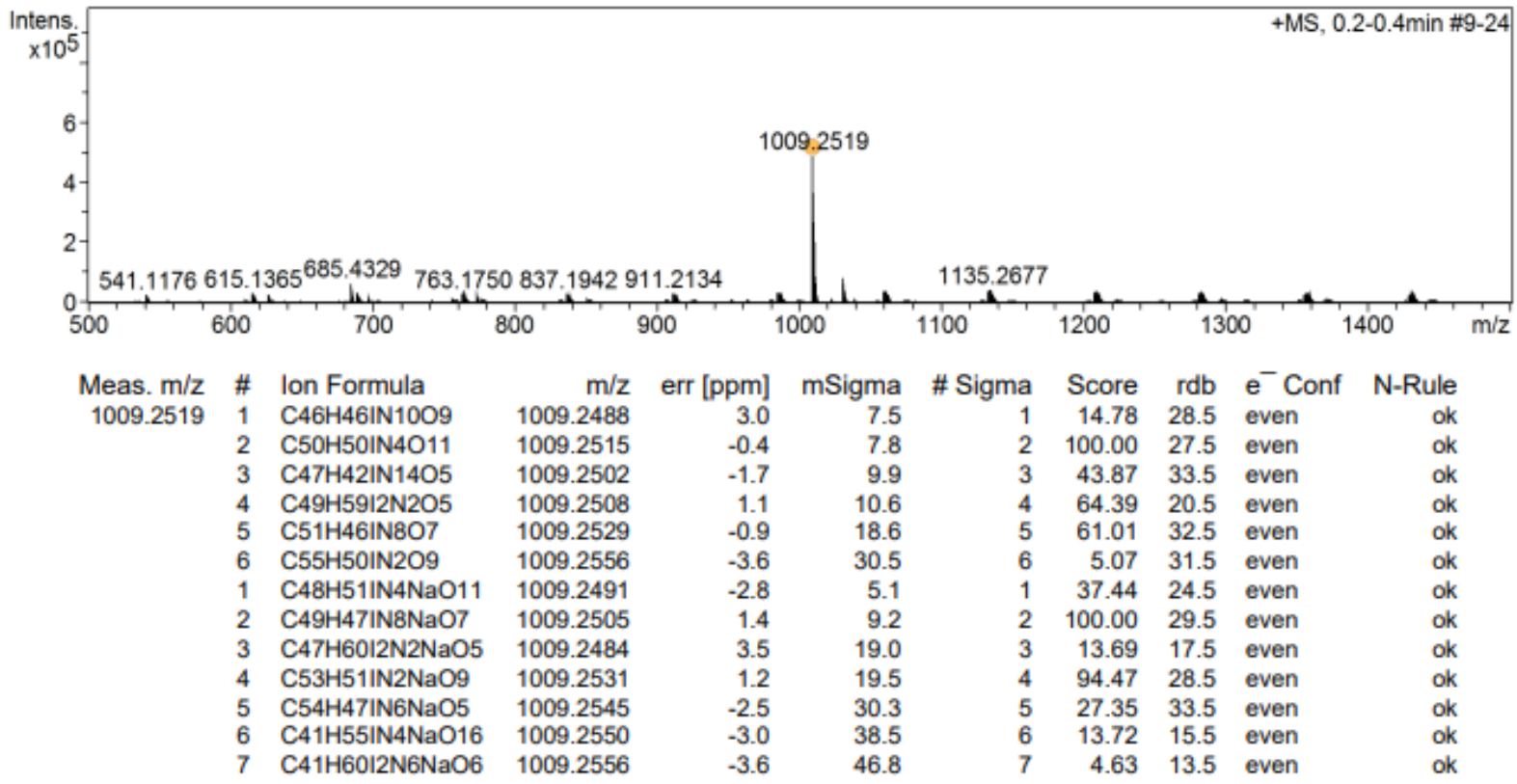

KDY007-1.d

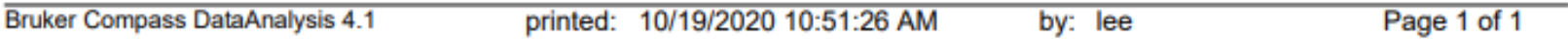

HR-MS of compound 13b 

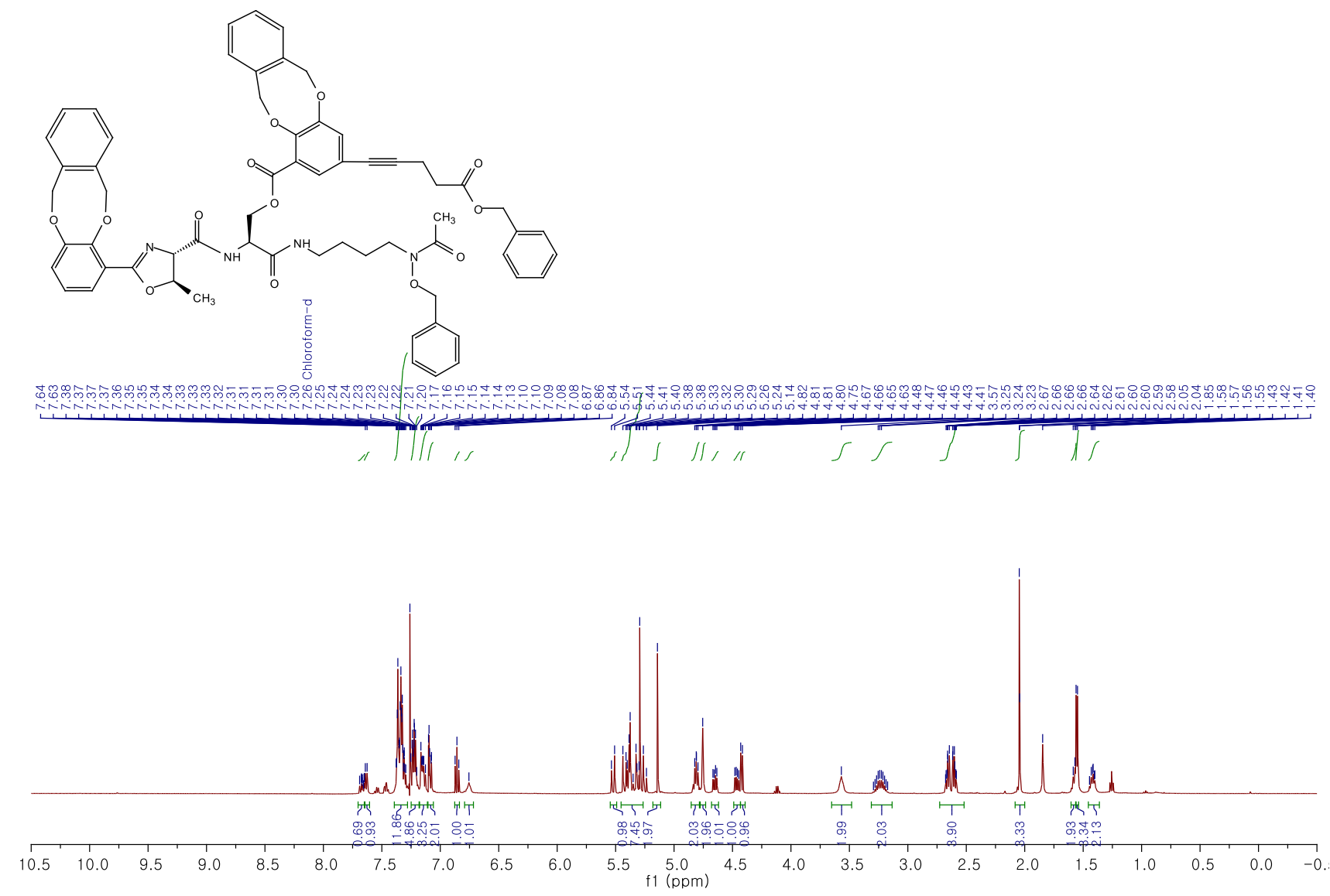

${ }^{1} \mathrm{H}-\mathrm{NMR}$ of compound $\mathbf{1 6 b}\left(500 \mathrm{MHz}, \mathrm{CDCl}_{3}\right)$

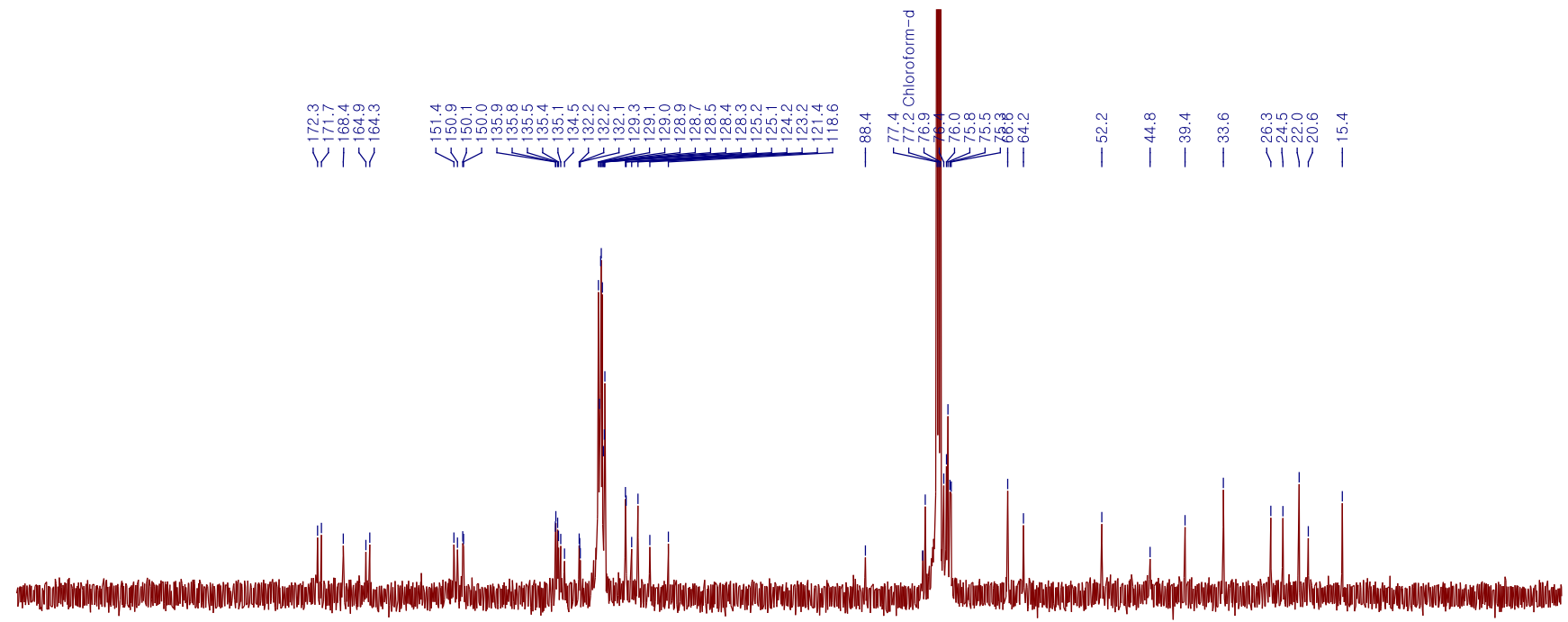

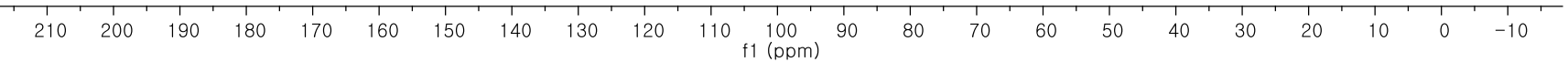

${ }^{13} \mathrm{C}-\mathrm{NMR}$ of compound $\mathbf{1 6 b}\left(125 \mathrm{MHz}, \mathrm{CDCl}_{3}\right)$ 


\section{Compound Spectrum SmartFormula Report}

Analysis Info

Analysis Name

Method

Sample Name

Comment
Acquisition Date 4/26/2021 7:02:32 PM

D:IDatalMCCBIKDY2104261210426-KDY-E12 intenal calibration.d

NaFormate_50_1600_MS method_POS.m

E12

Operator

Instrument

BDAL@DE

compact

8255754.20060

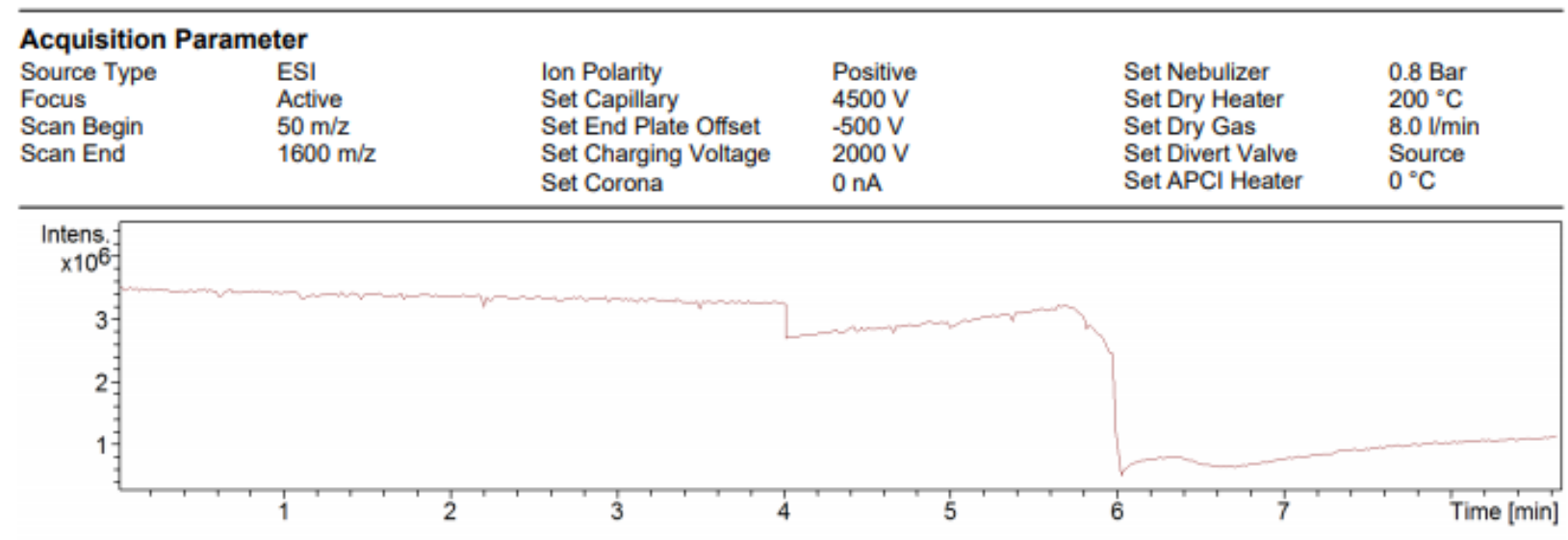

+MS, 6.3min \#368

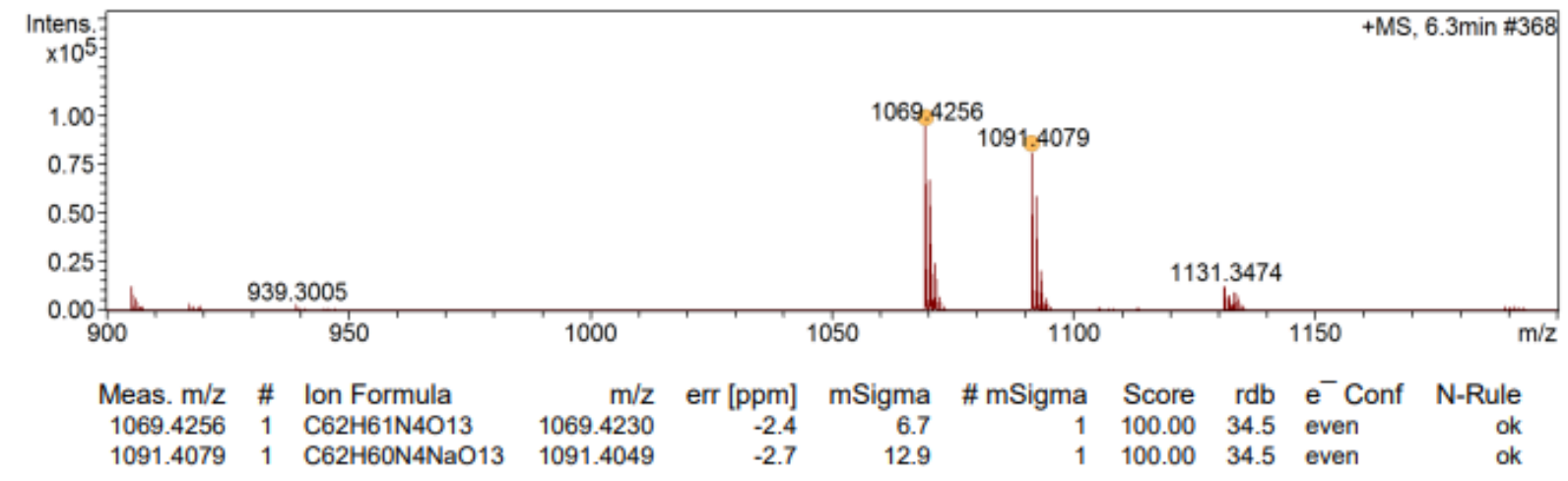

210426-KDY-E12 intenal calibration.d

Bruker Compass DataAnalysis 4.3

printed: 4/26/2021 7:21:58 PM

by: BDAL@DE

Page 1 of 1

HR-MS of compound $\mathbf{1 6 b}$ 


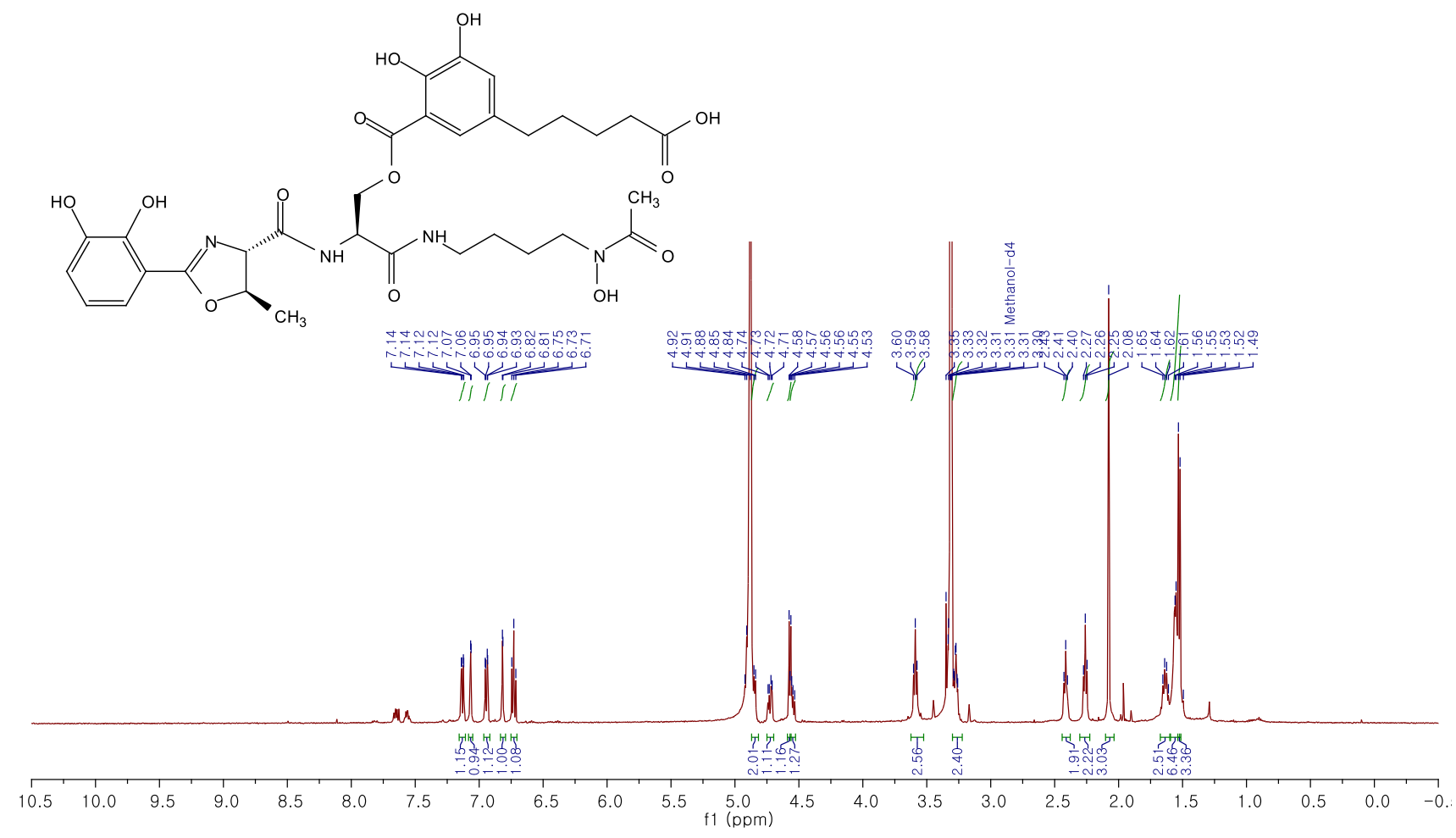

${ }^{1} \mathrm{H}-\mathrm{NMR}$ of compound $\mathbf{S} 7\left(500 \mathrm{MHz}, \mathrm{CD}_{3} \mathrm{OD}\right)$
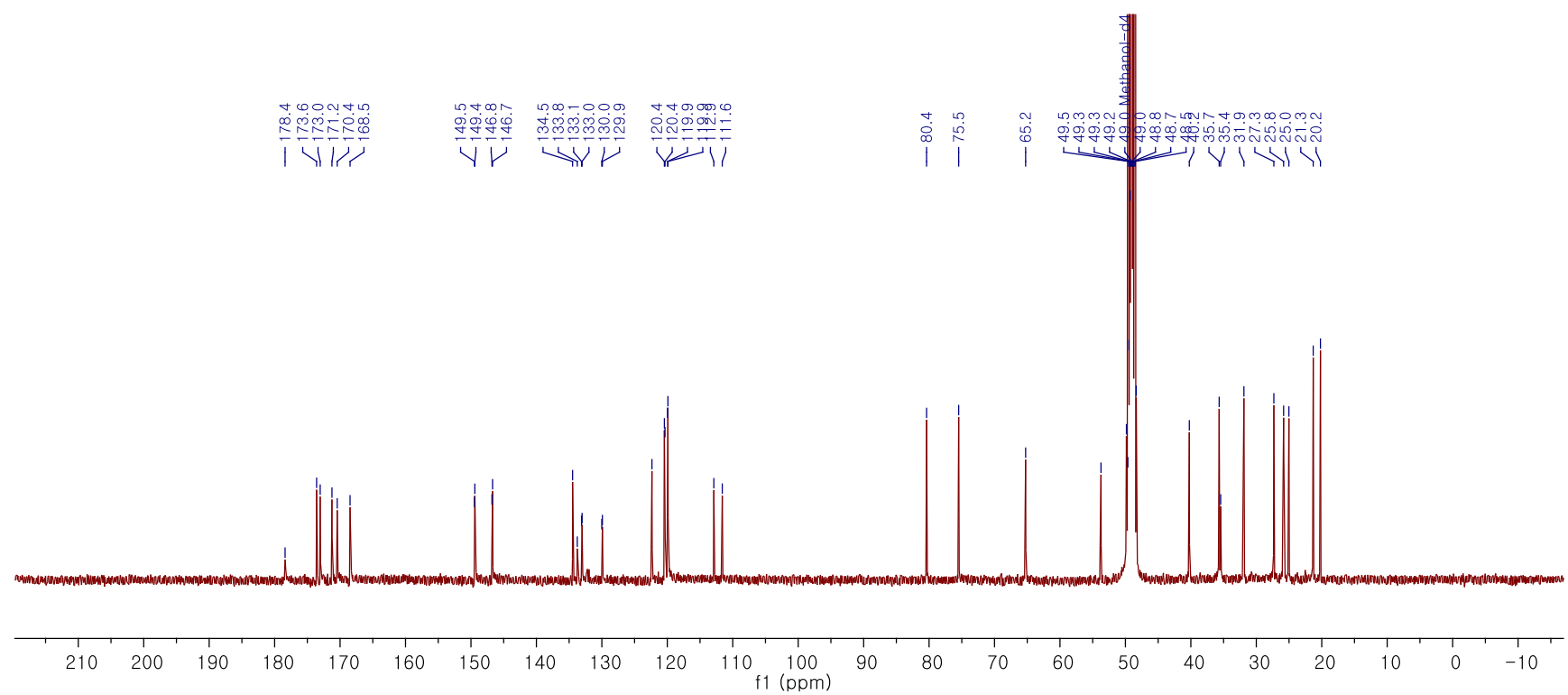

${ }^{13} \mathrm{C}$-NMR of compound $\mathbf{S} 7\left(125 \mathrm{MHz}, \mathrm{CD}_{3} \mathrm{OD}\right)$ 


\section{Compound Spectrum SmartFormula Report}

Analysis Info

Analysis Name Method

Sample Name

Comment
Acquisition Date 4/26/2021 9:33:34 PM

D:IDatalMCCBIKDY2104261210426-KDY-E13.d

NaFormate_50_1600_MS method_POS.m

E13
Operator BDAL@DE

Instrument compact

8255754.20060

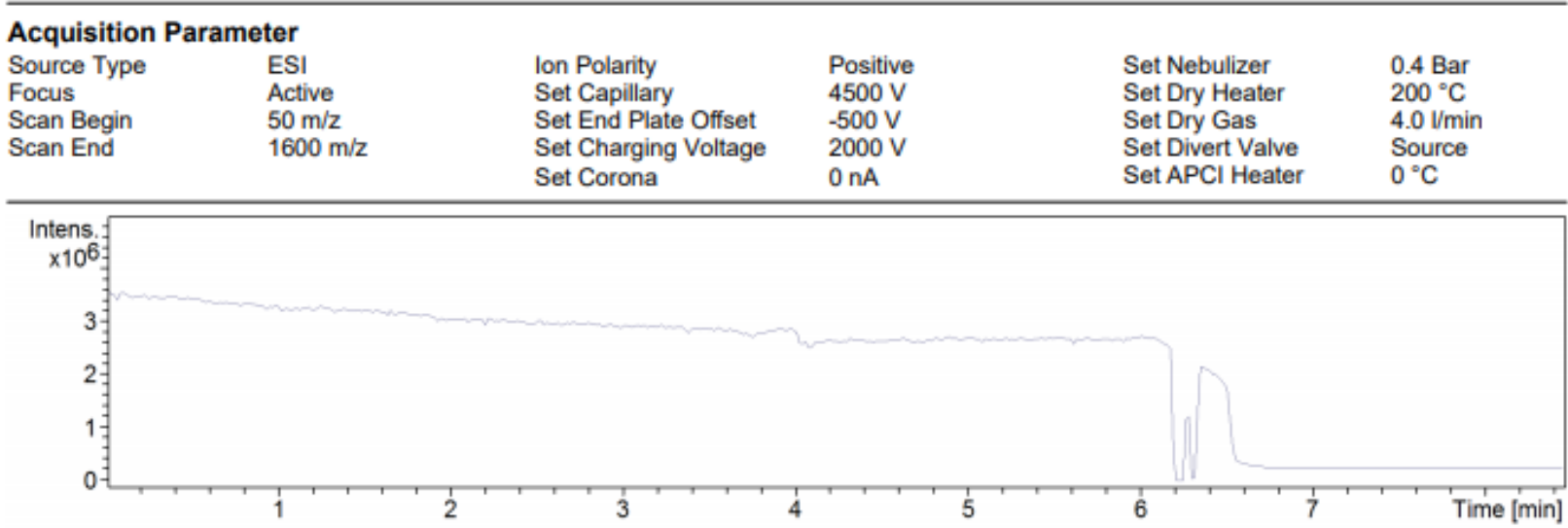

+MS, 6.7-6.8min \#393-398

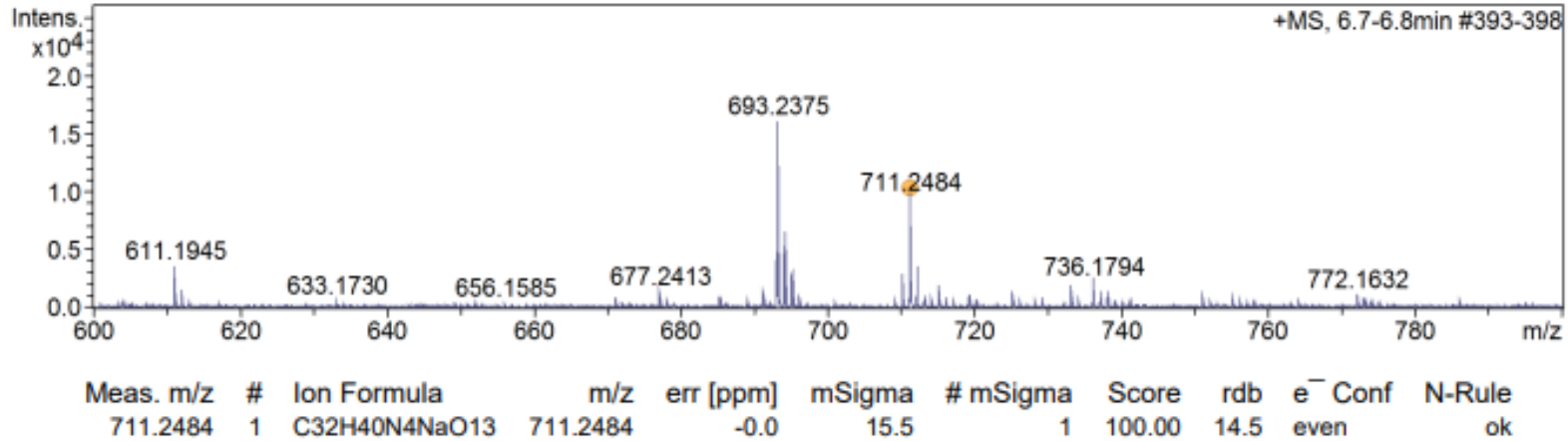

HR-MS of compound $\mathbf{S 7}$ 


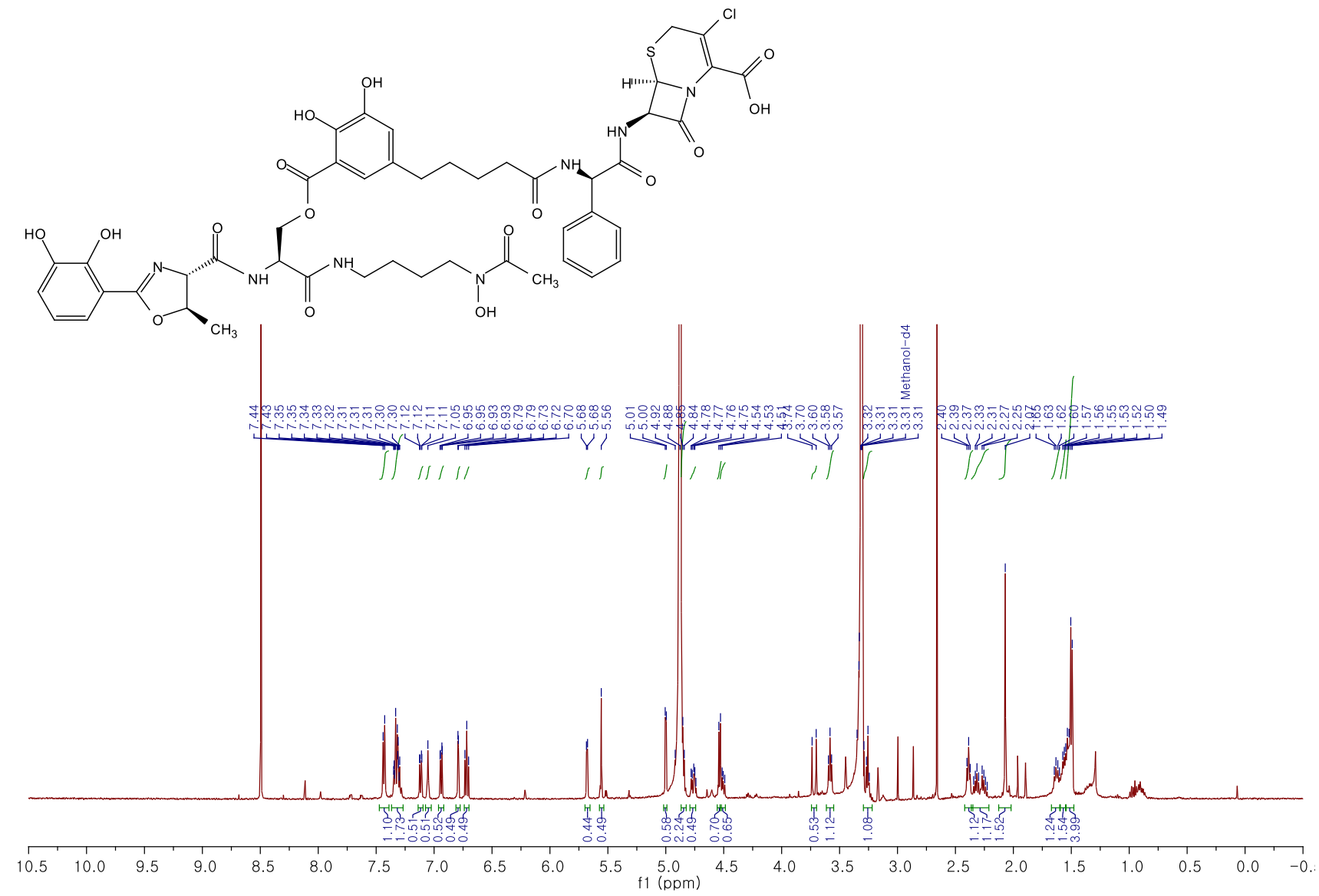

${ }^{1} \mathrm{H}-\mathrm{NMR}$ of compound $\mathbf{1 8 b}\left(500 \mathrm{MHz}, \mathrm{CD}_{3} \mathrm{OD}\right)$

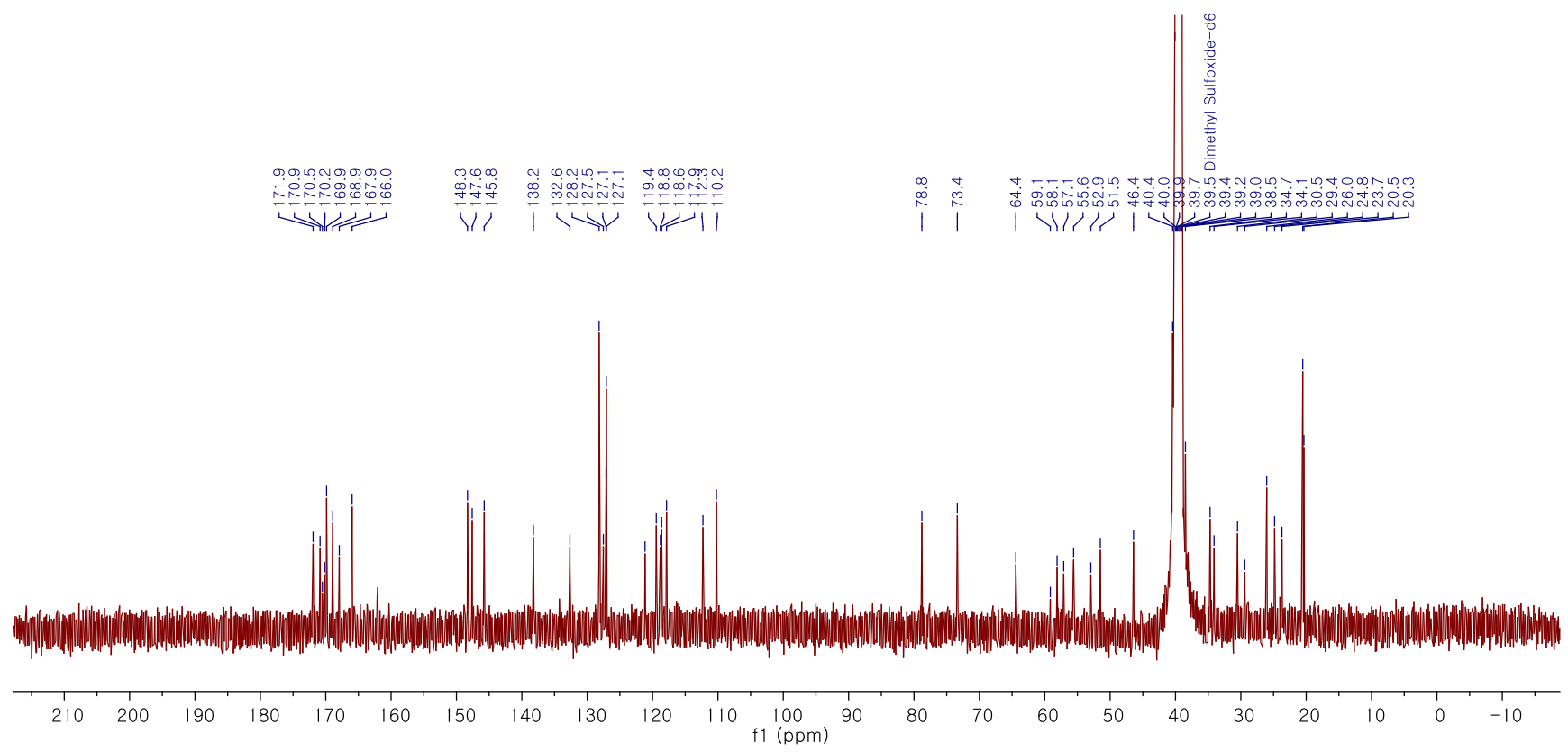

${ }^{13} \mathrm{C}-\mathrm{NMR}$ of compound $\mathbf{1 8 b}\left(125 \mathrm{MHz}, \mathrm{DMSO}-\mathrm{d}^{6}\right)$ 


\section{Compound Spectrum SmartFormula Report}

Analysis Info

Analysis Name

Method

Sample Name

Comment
Acquisition Date 3/26/2021 10:34:26 AM

D:IDatalMCCBIKDY2103261210326_KDY_FimB-Cec-Es_internal calib.d

ms method 0.8 and $8.0 . \mathrm{m}$

wash

Operator

Instrument

BDAL@DE

compact

8255754.20060

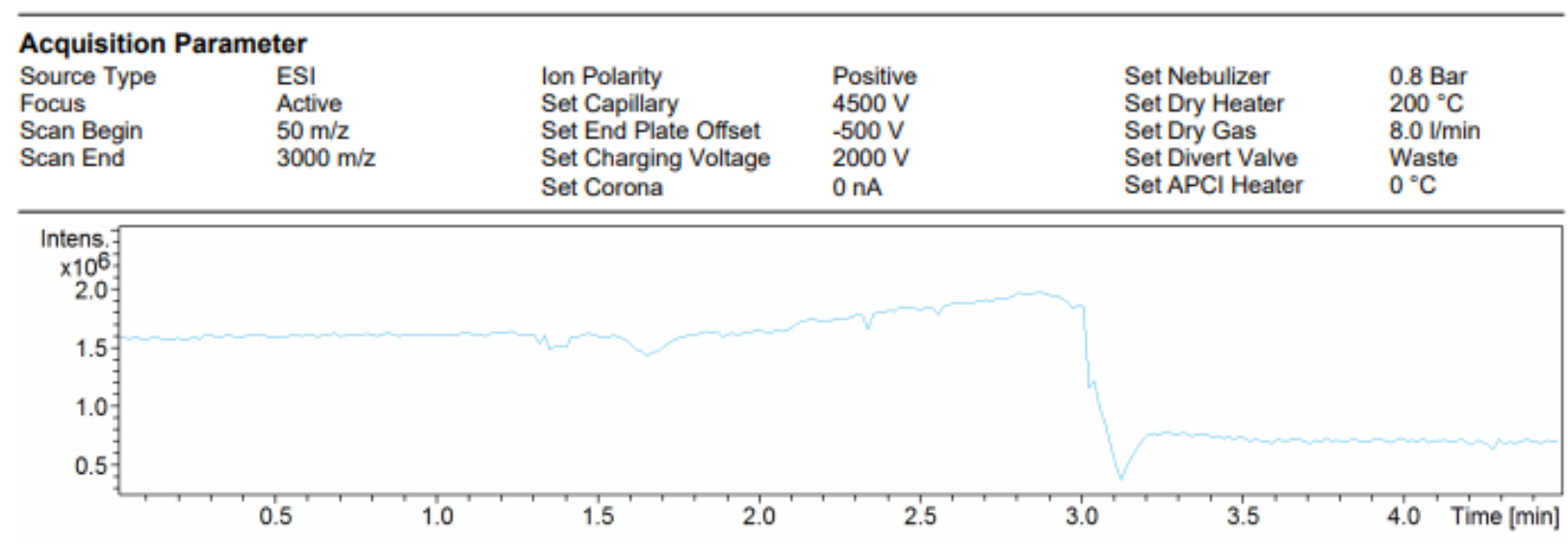

+MS, 4.2-4.2min \#253-254

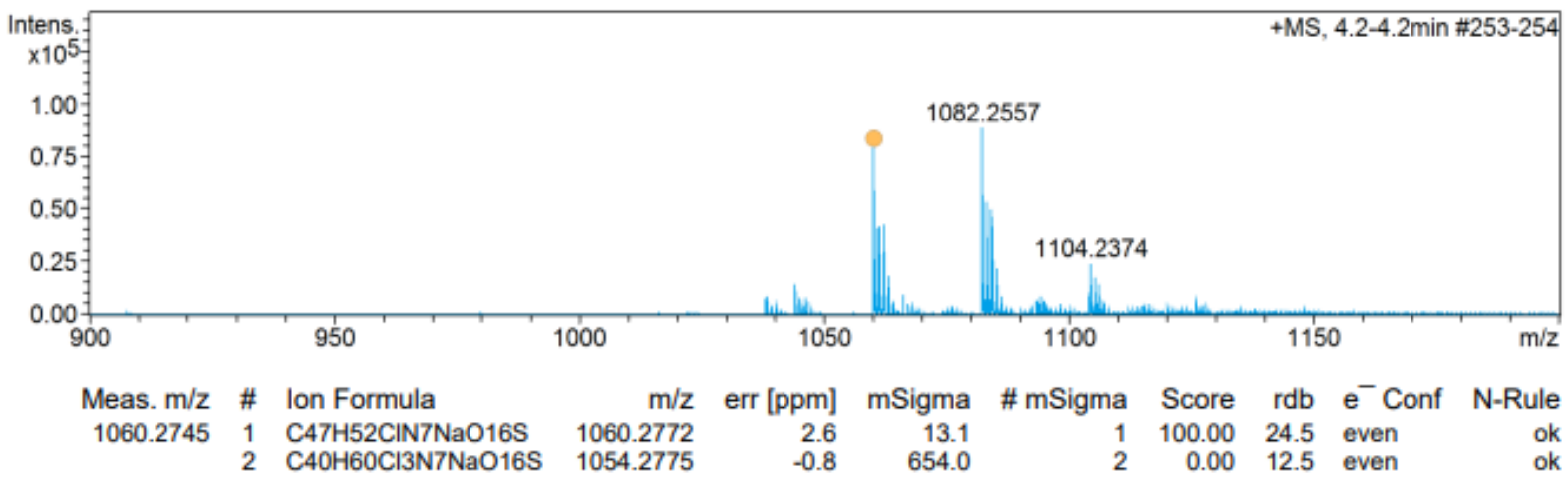

210326_KDY_FimB-Cec-Es_internal calib.d

HR-MS of compound $\mathbf{1 8 b}$ 


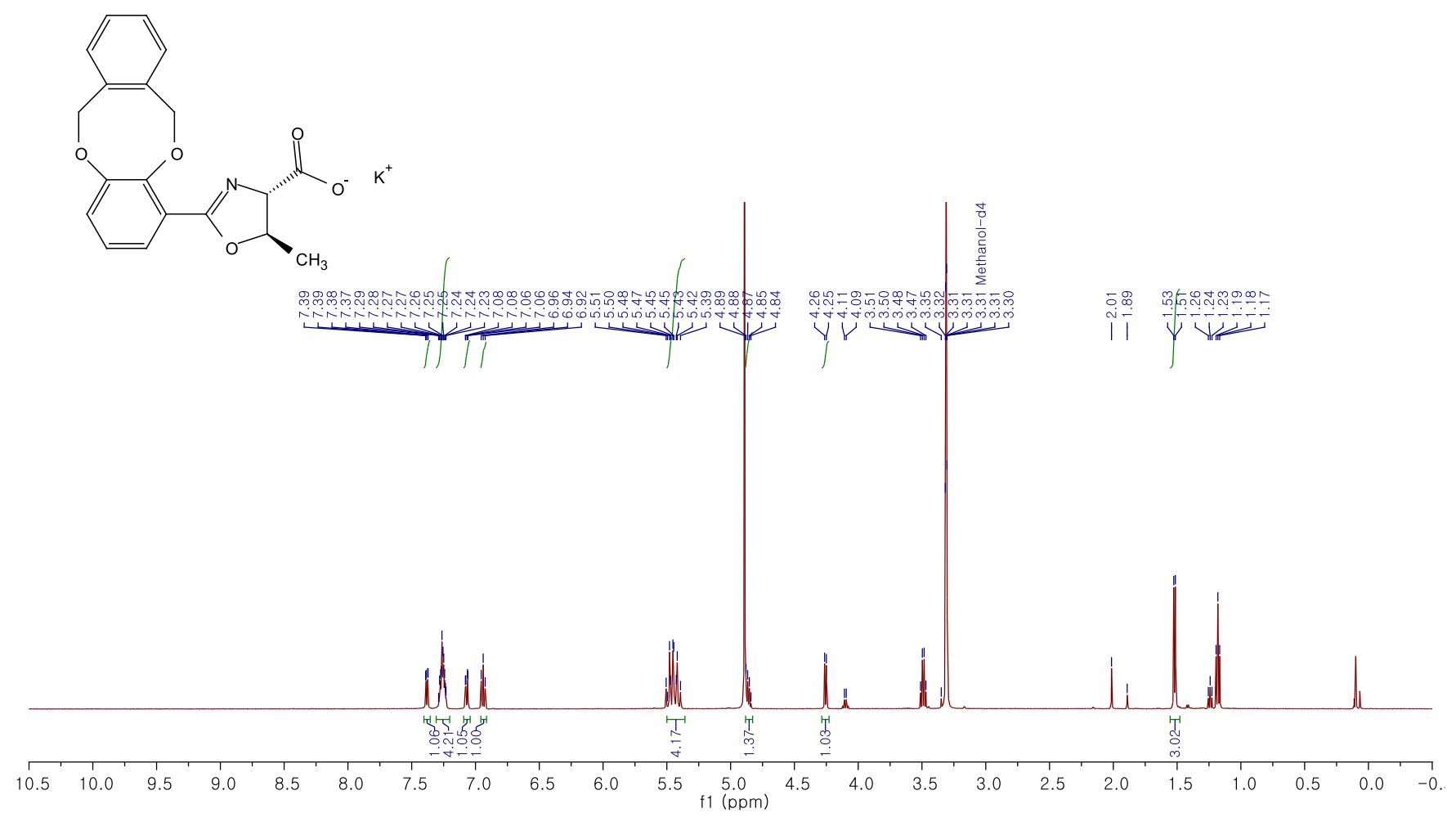

${ }^{1} \mathrm{H}-\mathrm{NMR}$ of compound $\mathbf{8 b}\left(500 \mathrm{MHz}, \mathrm{CD}_{3} \mathrm{OD}\right)$ 

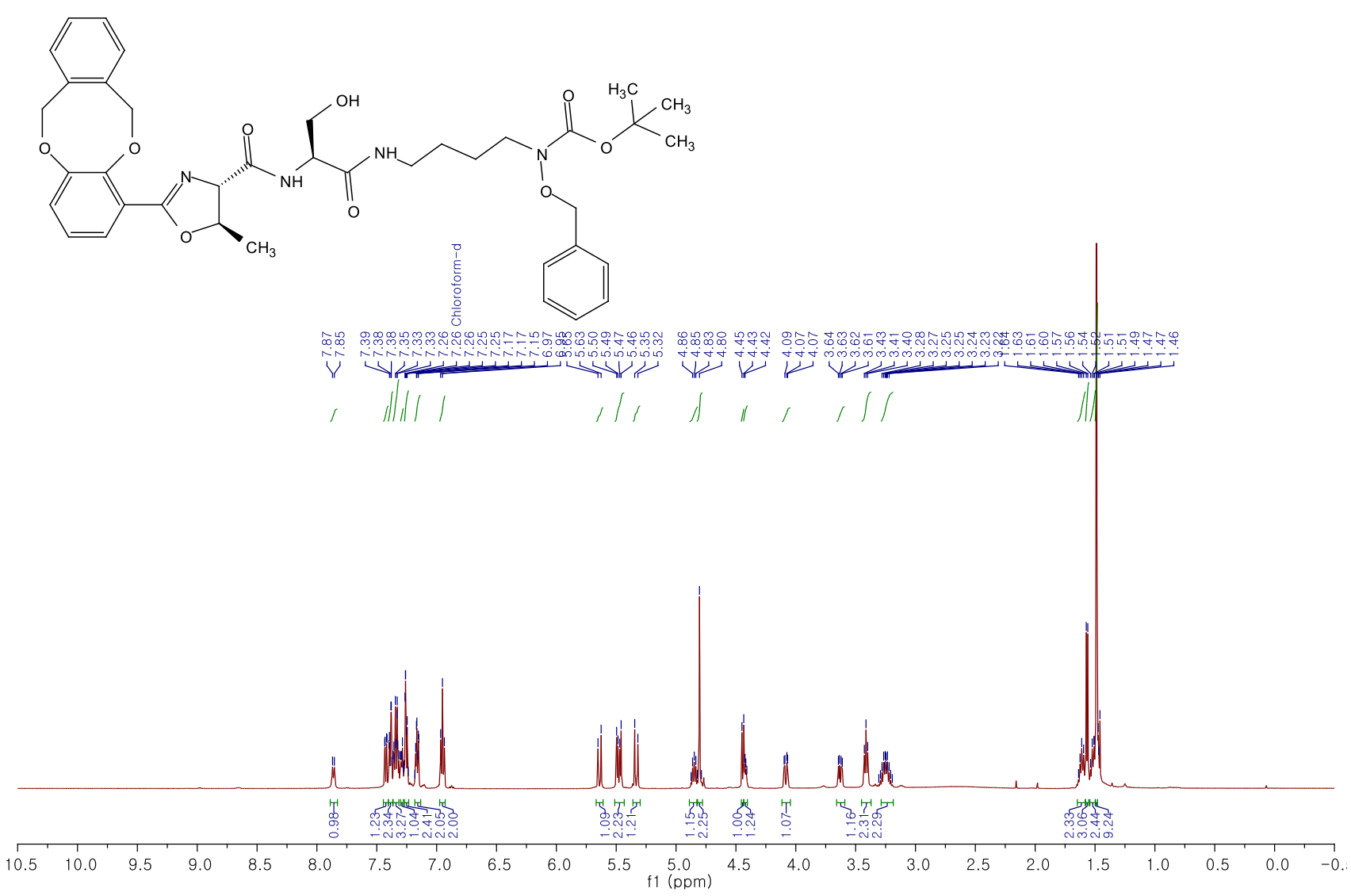

${ }^{1} \mathrm{H}-\mathrm{NMR}$ of compound $11 \mathrm{c}\left(500 \mathrm{MHz}, \mathrm{CDCl}_{3}\right)$

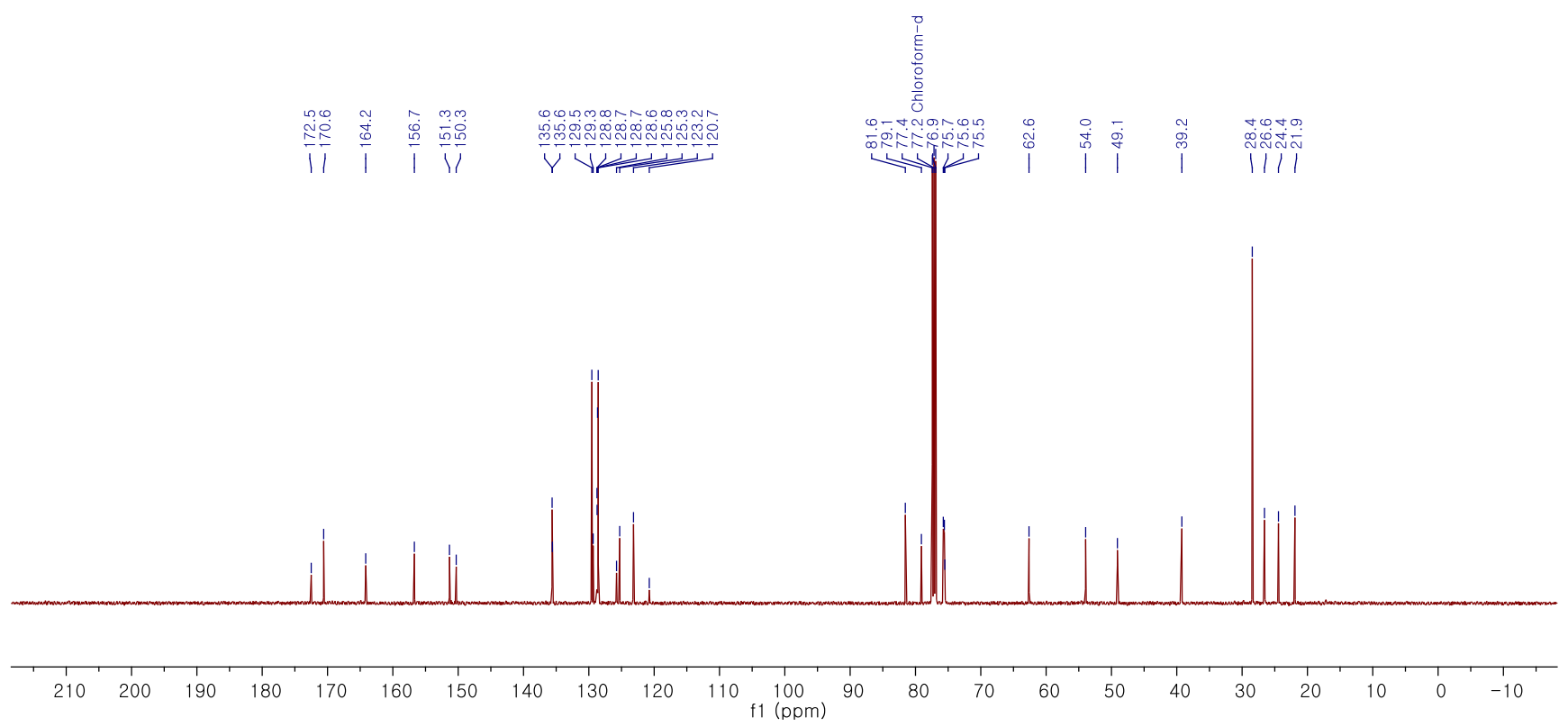

${ }^{13} \mathrm{C}-\mathrm{NMR}$ of compound $11 \mathrm{c}\left(125 \mathrm{MHz}, \mathrm{CDCl}_{3}\right)$ 


\section{Compound Spectrum SmartFormula Report}

\section{Analysis Info}

Analysis Name

Method

Sample Name

Comment
D:IDatalDatal2020IKUVKHJKDY10IKDY004.d

Tune_pos_Standard_50_600_20201008.d.m

DY4-4c
Acquisition Date $\quad 10 / 15 / 2020$ 10:44:52 AM

Operator lee

Instrument compact

\begin{tabular}{llllll}
\hline \multicolumn{2}{l}{ Acquisition Parameter } & & & & \\
Source Type & ESI & Ion Polarity & Positive & Set Nebulizer & 0.3 Bar \\
Focus & Active & Set Capillary & $4500 \mathrm{~V}$ & Set Dry Heater & $200^{\circ} \mathrm{C}$ \\
Scan Begin & $50 \mathrm{~m} / \mathrm{z}$ & Set End Plate Offset & $-500 \mathrm{~V}$ & Set Dry Gas & $4.0 \mathrm{~V} / \mathrm{min}$ \\
Scan End & $1000 \mathrm{~m} / \mathrm{z}$ & Set Charging Voltage & $2000 \mathrm{~V}$ & Set Divert Valve & Source \\
& & Set Corona & $0 \mathrm{nA}$ & Set APCl Heater & $0^{\circ} \mathrm{C}$
\end{tabular}

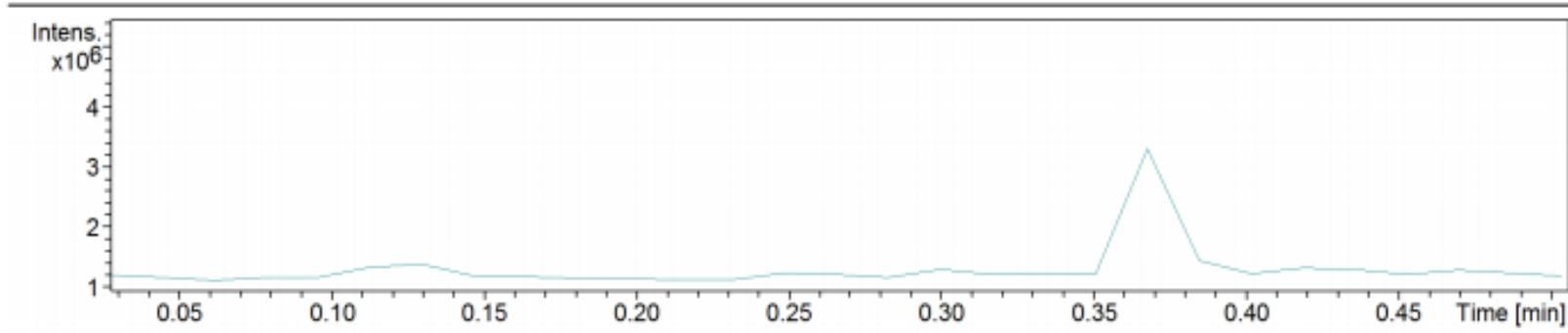

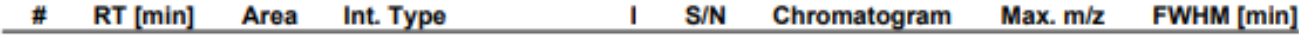

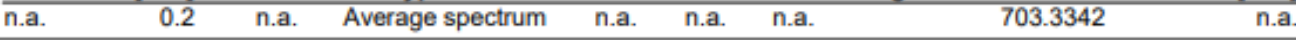

+MS, 0.2-0.3min \#13-15

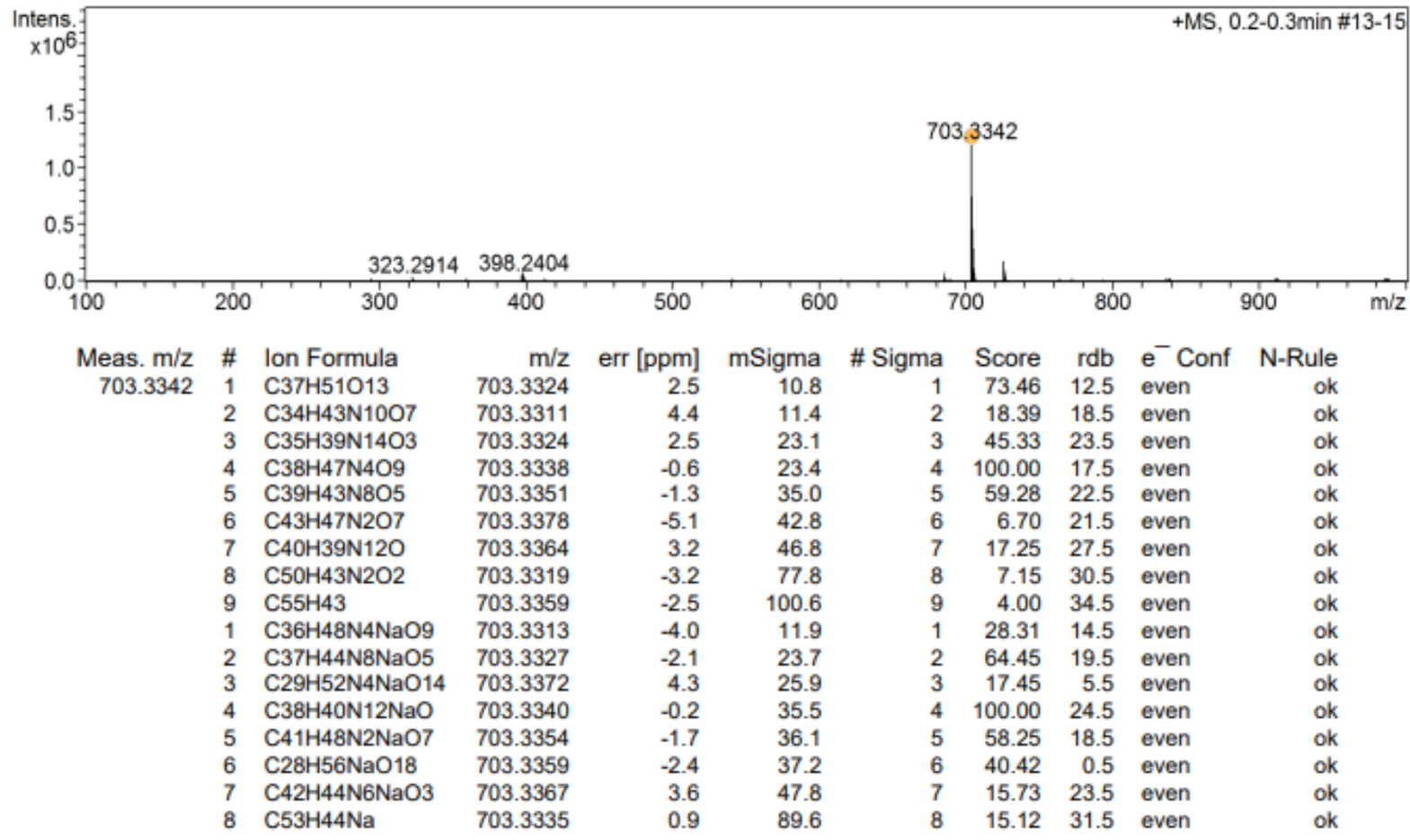

KDY004.d

HR-MS of compound 11c 


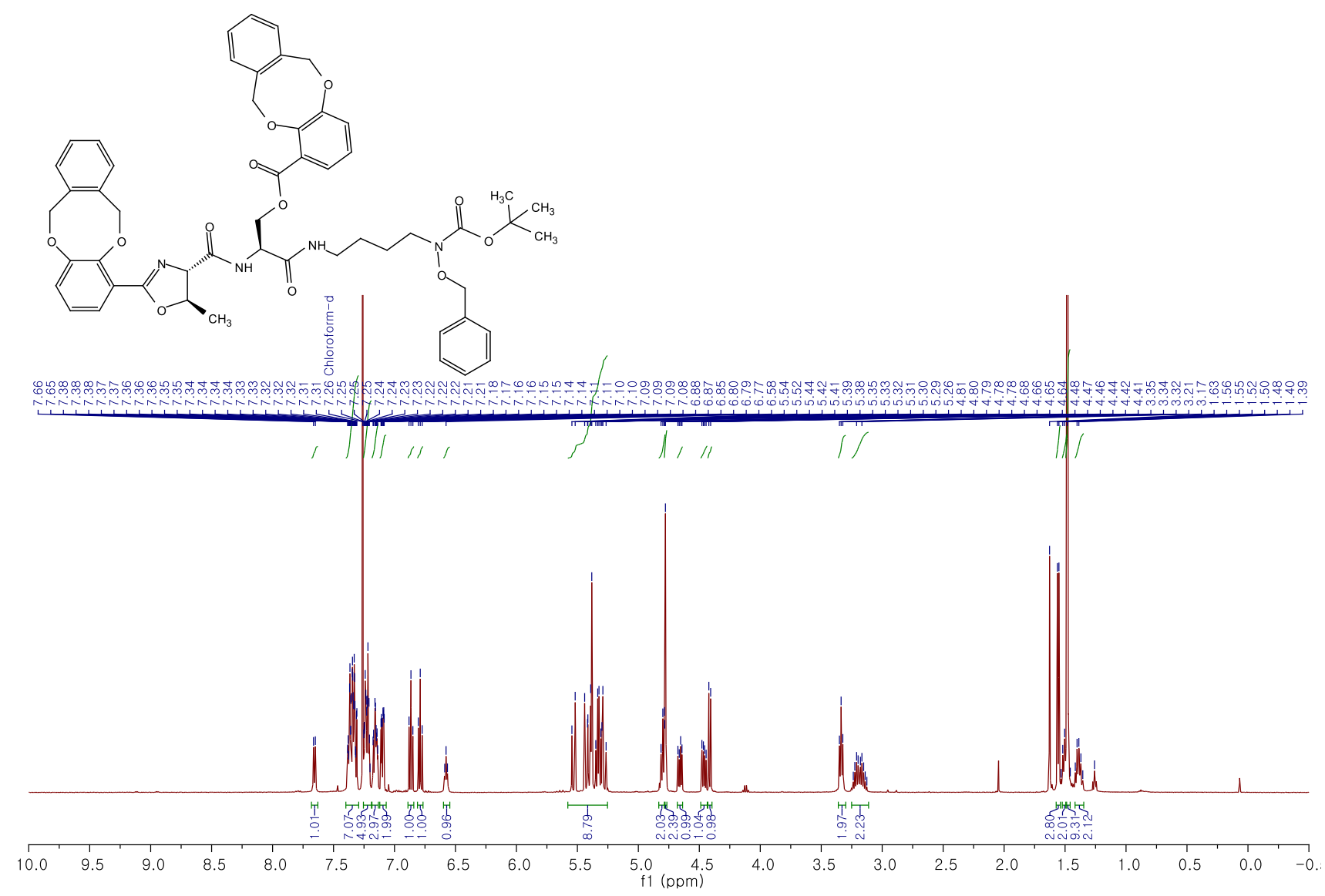

${ }^{1} \mathrm{H}-\mathrm{NMR}$ of compound $\mathbf{1 3 c}\left(500 \mathrm{MHz}, \mathrm{CDCl}_{3}\right)$

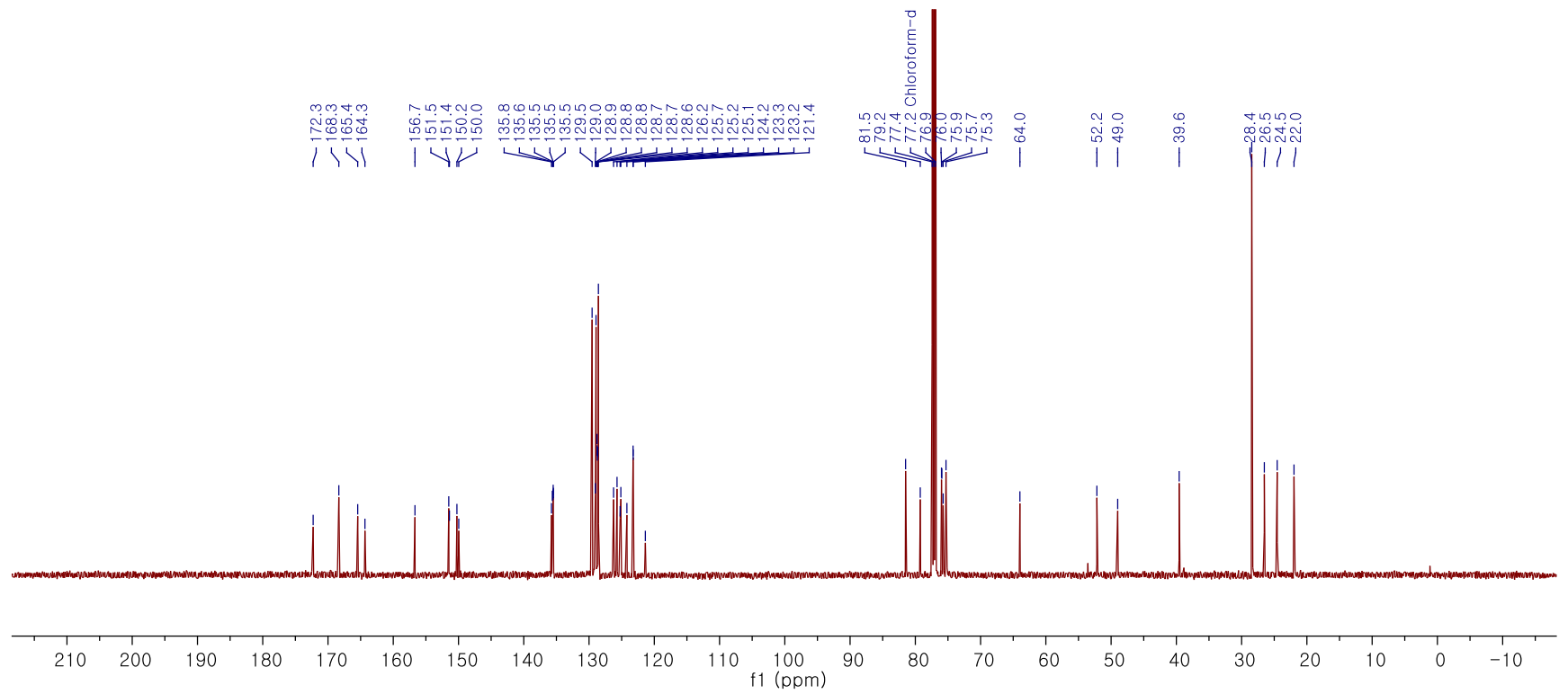

${ }^{13} \mathrm{C}-\mathrm{NMR}$ of compound $\mathbf{1 3 c}\left(125 \mathrm{MHz}, \mathrm{CDCl}_{3}\right)$ 


\section{Compound Spectrum SmartFormula Report}

\section{Analysis Info}

Analysis Name

Method

Sample Name

D:IDatalDatal20201KUKKJJKDY10IKDY009-2.d

Tune_pos_Standard_50_600_20201008.d.m

DY9-6c
Acquisition Date 10/16/2020 3:22:26 PM

Operator lee

Instrument compact

8255754.10024

Comment

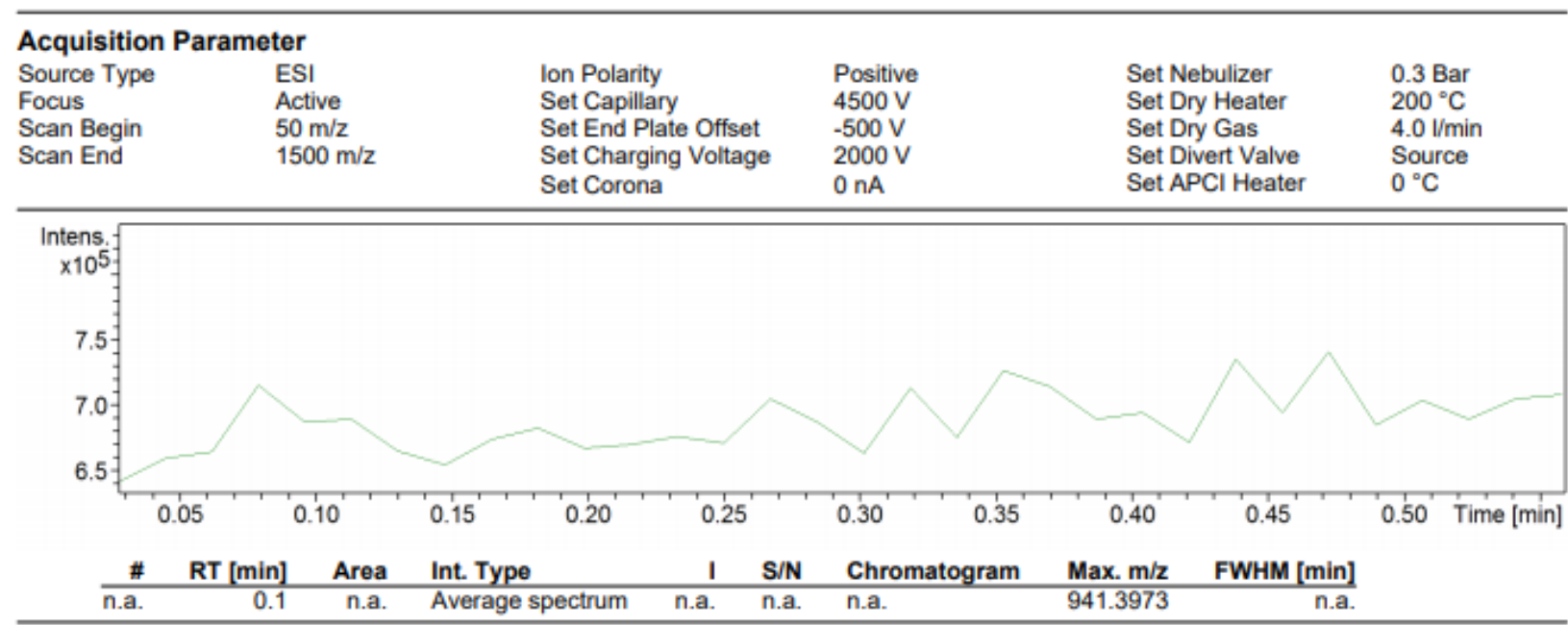

+MS, 0.1-0.2min \#4-12

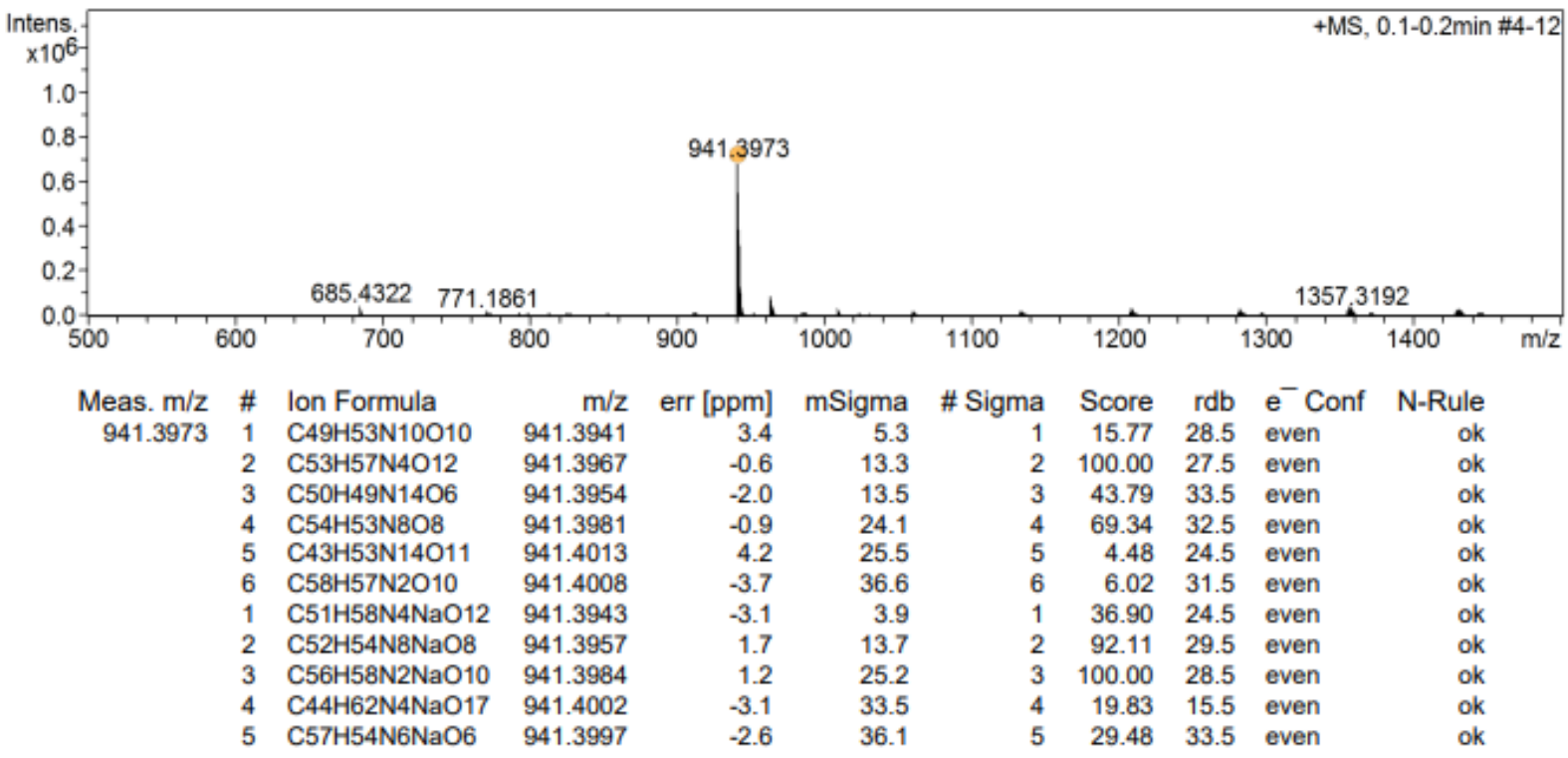

KDY009-2.d

HR-MS of compound 13c 


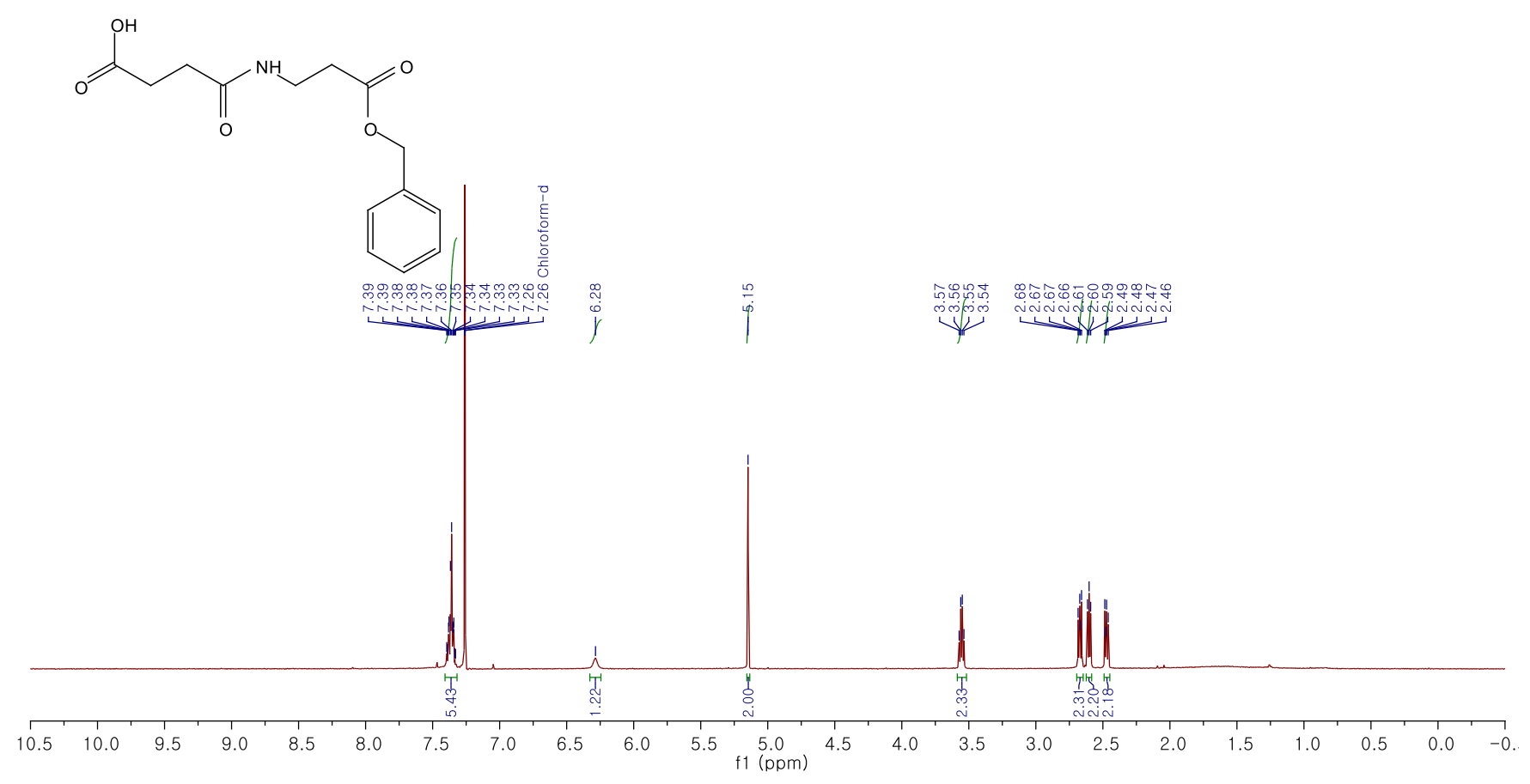

${ }^{1} \mathrm{H}-\mathrm{NMR}$ of compound $15\left(500 \mathrm{MHz}, \mathrm{CDCl}_{3}\right)$ 


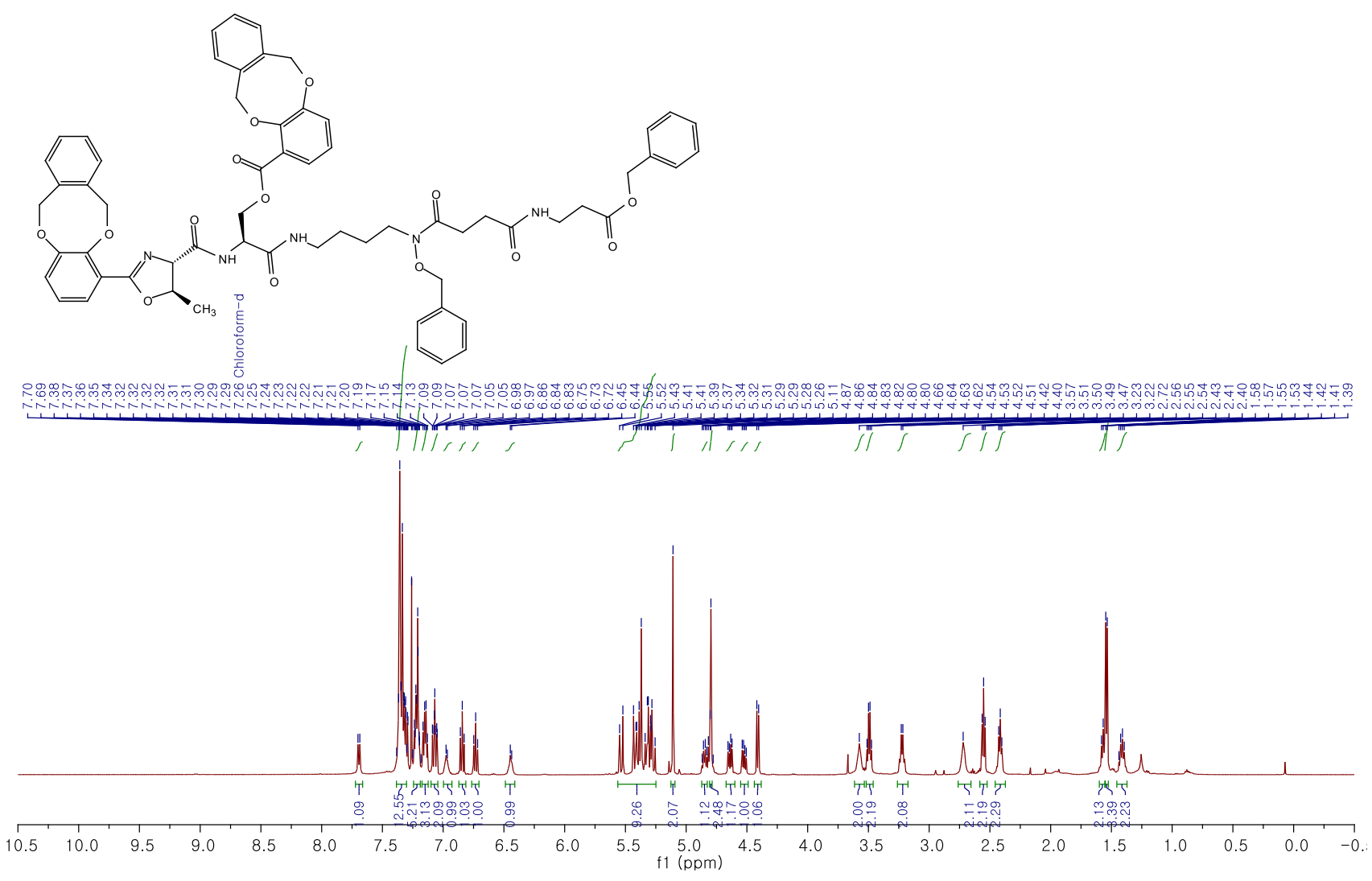

${ }^{1} \mathrm{H}-\mathrm{NMR}$ of compound $\mathbf{1 6 c}\left(500 \mathrm{MHz}, \mathrm{CDCl}_{3}\right)$

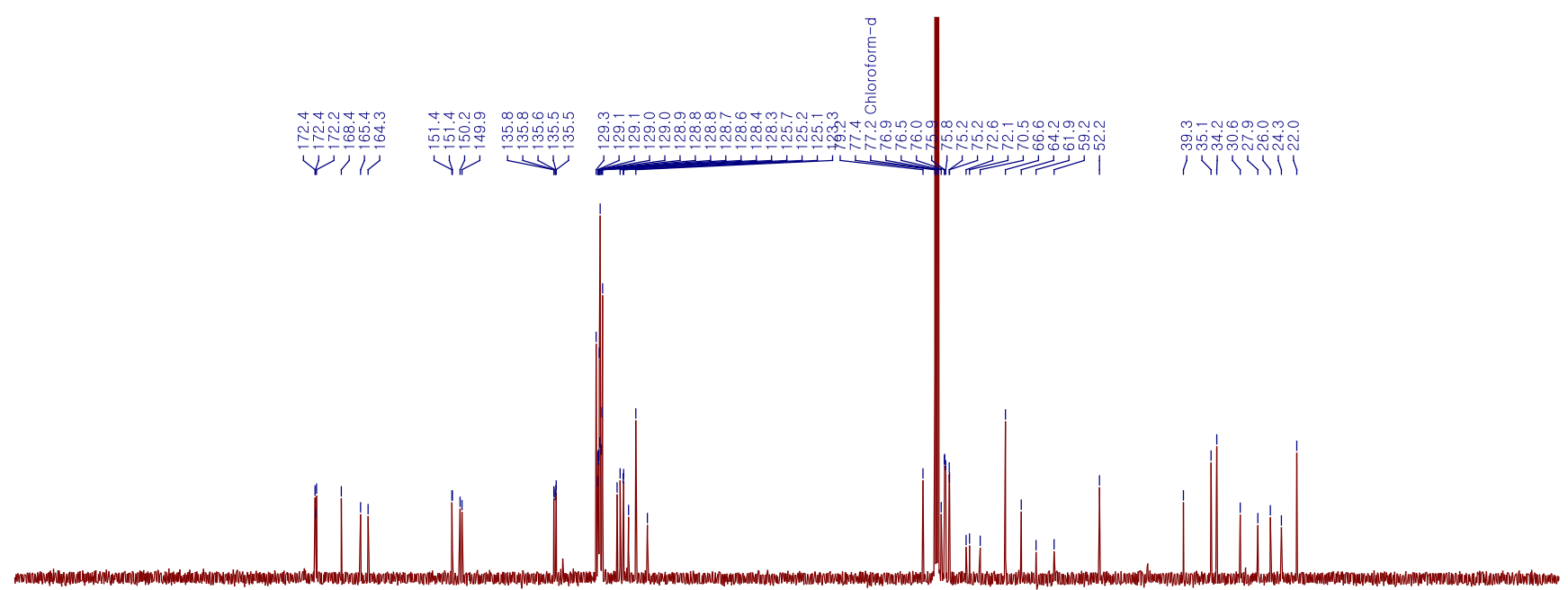

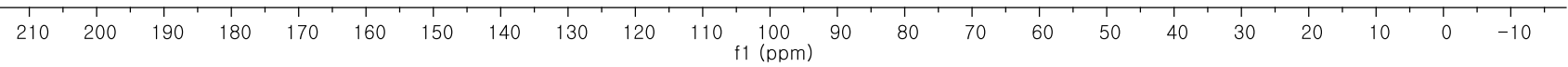

${ }^{13} \mathrm{C}-\mathrm{NMR}$ of compound $\mathbf{1 6 c}\left(125 \mathrm{MHz}, \mathrm{CDCl}_{3}\right)$ 


\section{Compound Spectrum SmartFormula Report}

\section{Analysis Info}

Analysis Name Method

Sample Name

Comment
Acquisition Date $\quad$ 10/16/2020 4:02:49 PM

D:IDatalDatal2020IKUIKHJKDY10IKDY024.d

Tune_pos_Standard_50_600_20201008.d.m

DY24-S19
Operator lee

Instrument compact

Acquisition Parameter

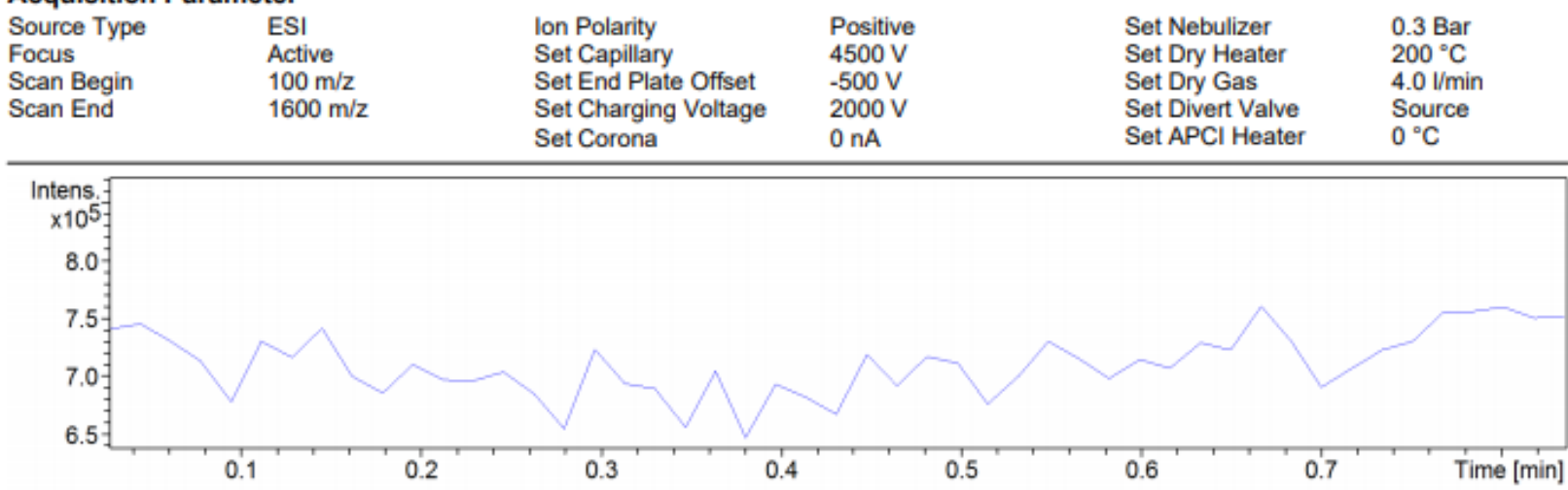

\# RT [min] Area Int. Type

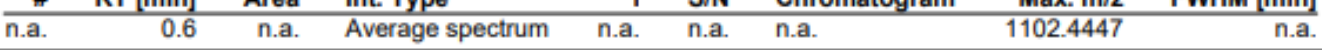

Max. $\mathrm{m} / \mathrm{z} \quad$ FWHM [min]

+MS, 0.5-0.7min \#27-41

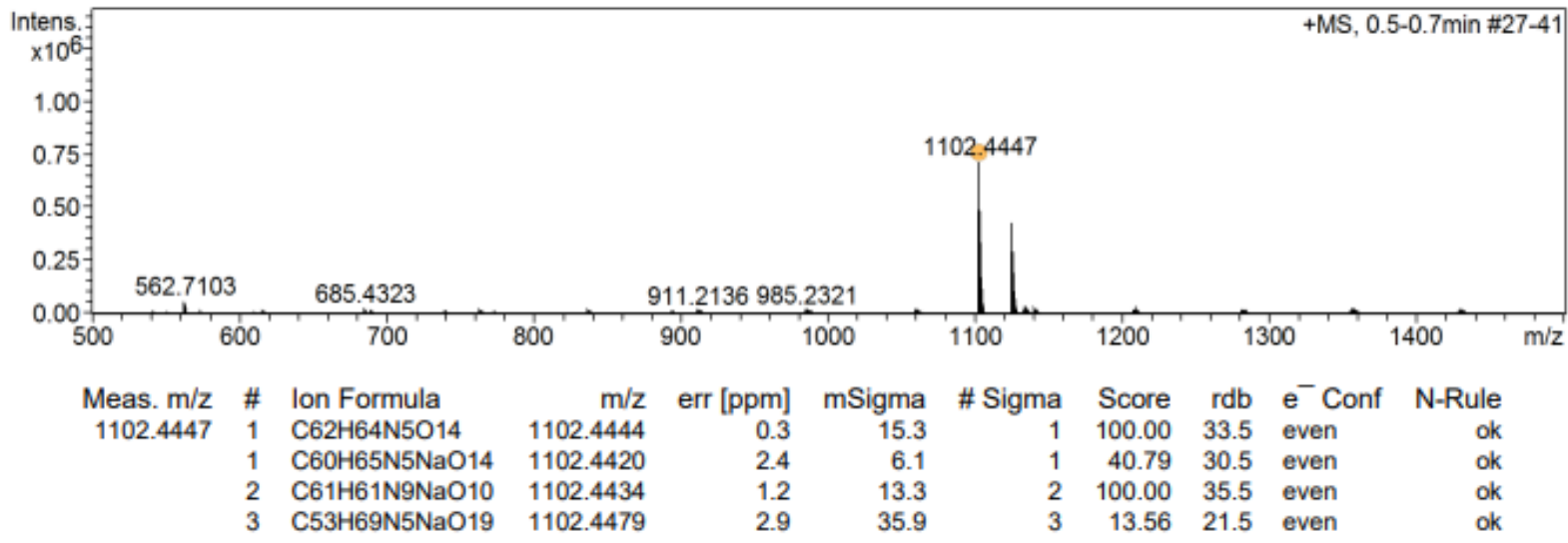

KDY024.d

HR-MS of compound $\mathbf{1 6 c}$ 


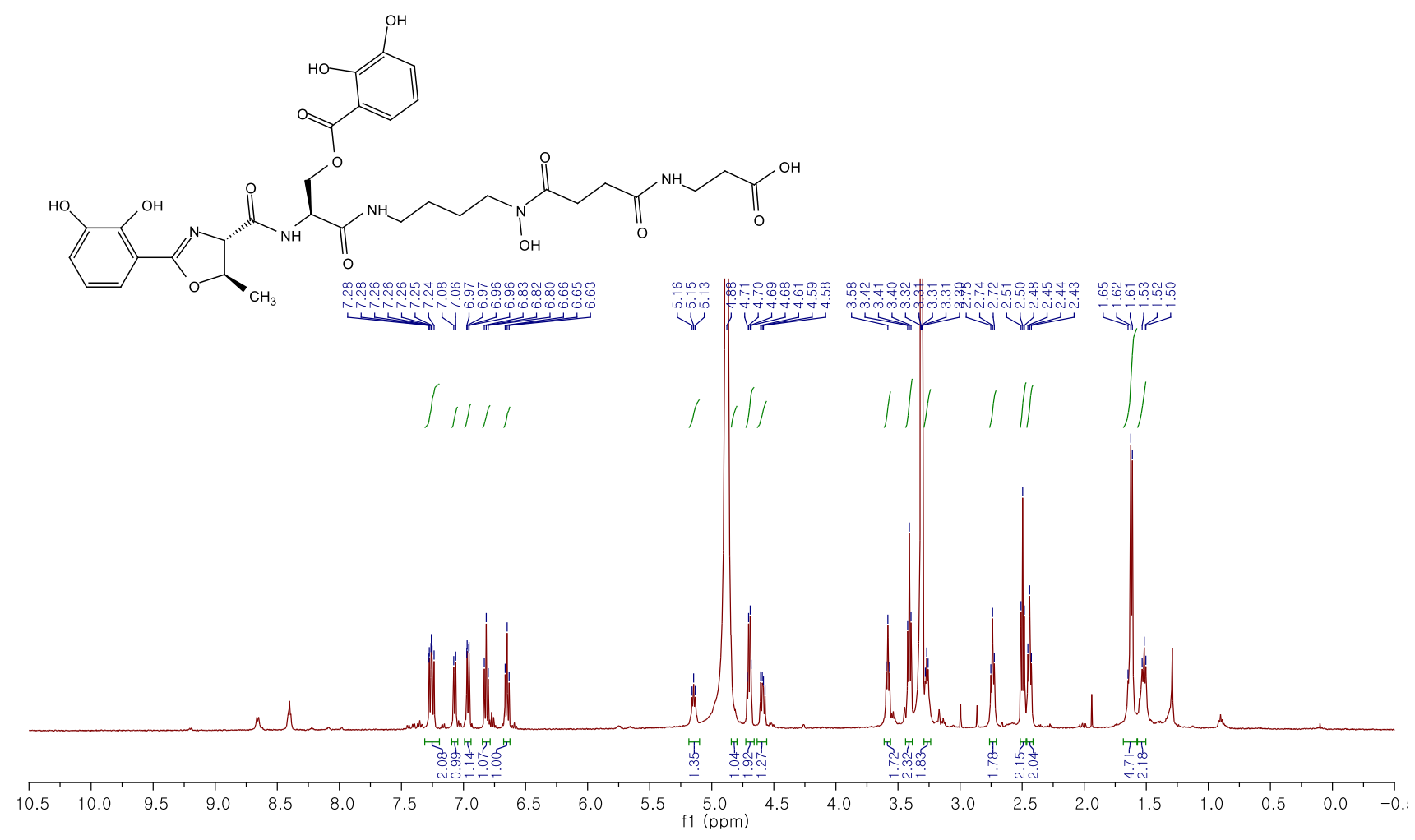

${ }^{1} \mathrm{H}-\mathrm{NMR}$ of compound $\mathbf{S 8}\left(500 \mathrm{MHz}, \mathrm{CD}_{3} \mathrm{OD}\right)$

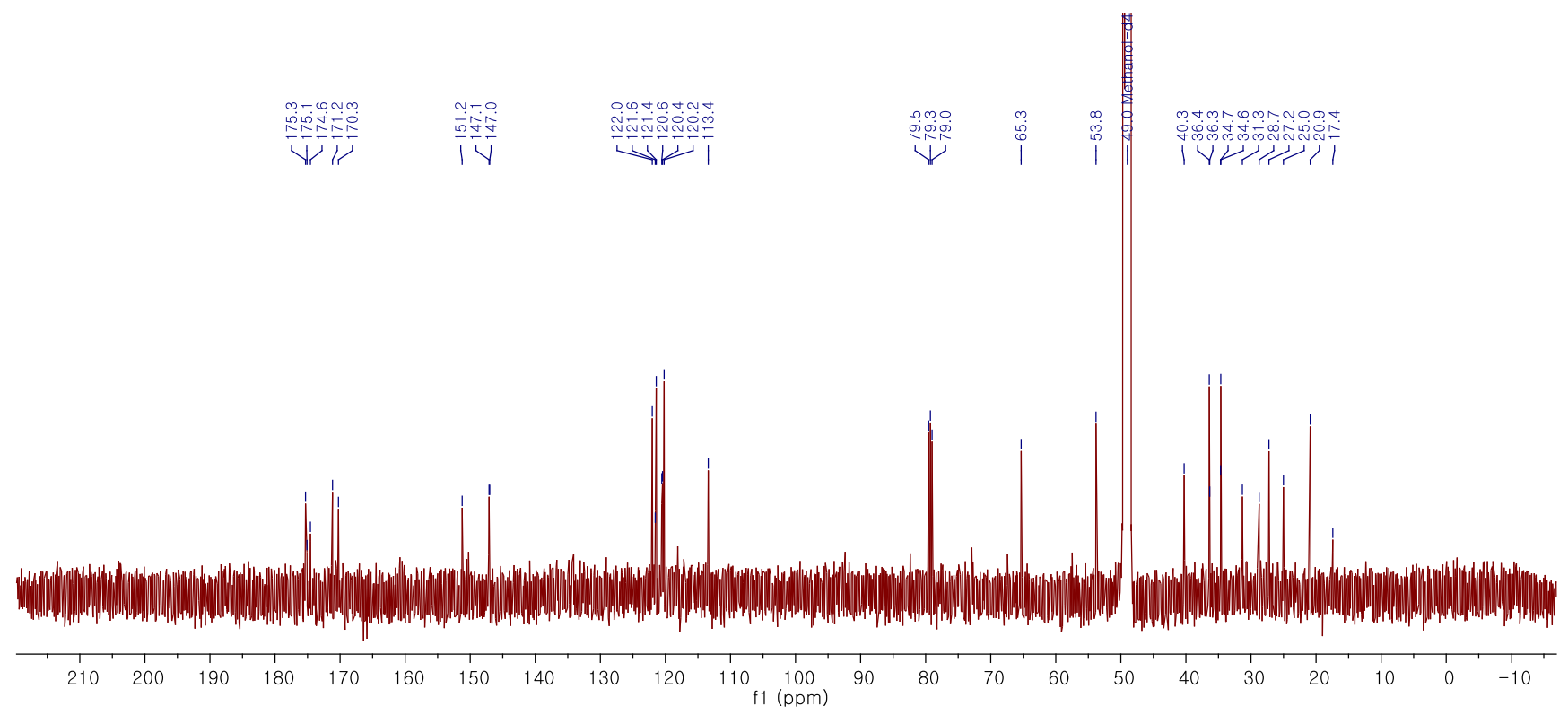

${ }^{13} \mathrm{C}-\mathrm{NMR}$ of compound $\mathbf{S 8}\left(125 \mathrm{MHz}, \mathrm{CD}_{3} \mathrm{OD}\right)$ 


\section{Compound Spectrum SmartFormula Report}

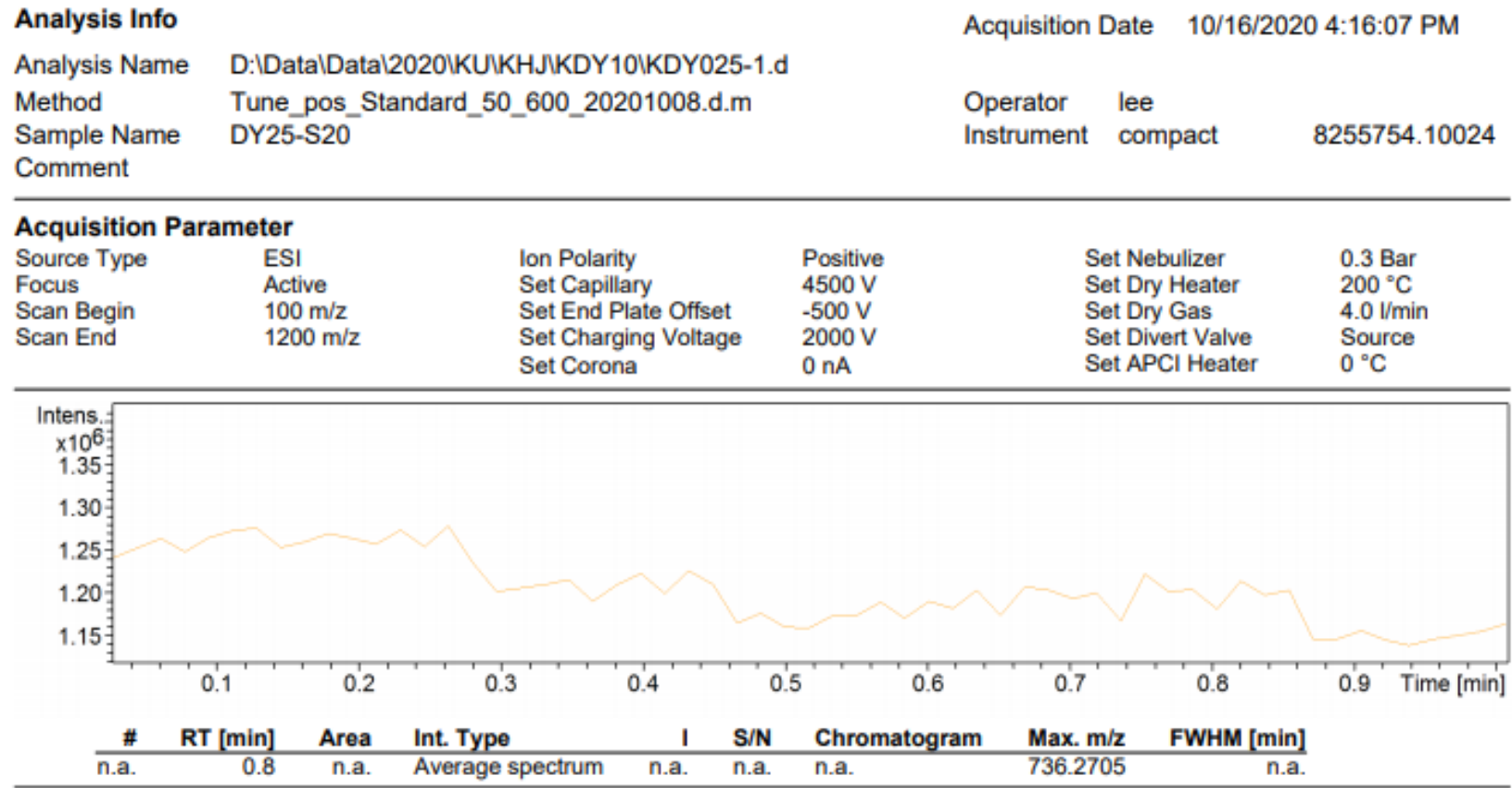

+MS, 0.7-0.9min \#39-55

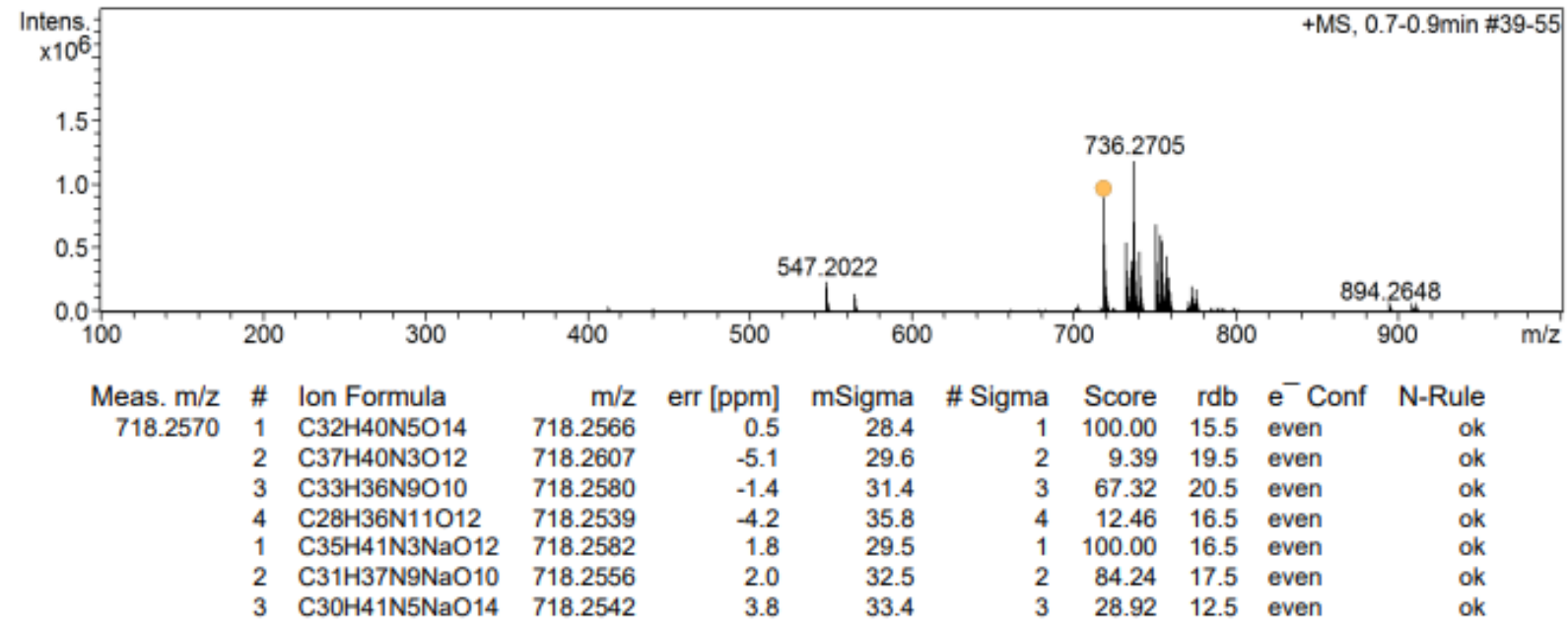

KDY025-1.d

HR-MS of compound $\mathbf{S 8}$ 


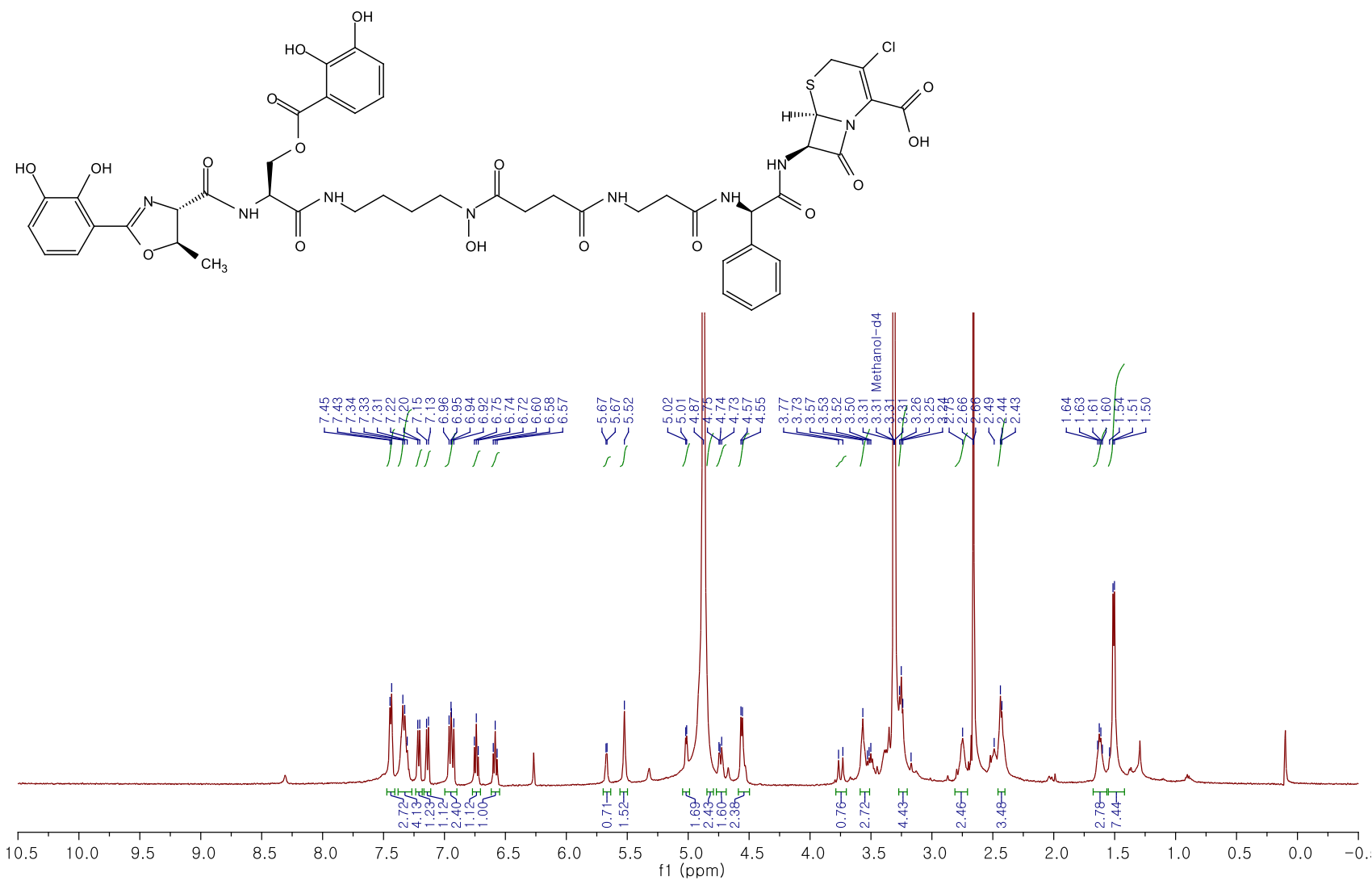

${ }^{1} \mathrm{H}-\mathrm{NMR}$ of compound $18 \mathrm{c}\left(500 \mathrm{MHz}, \mathrm{CD}_{3} \mathrm{OD}\right)$

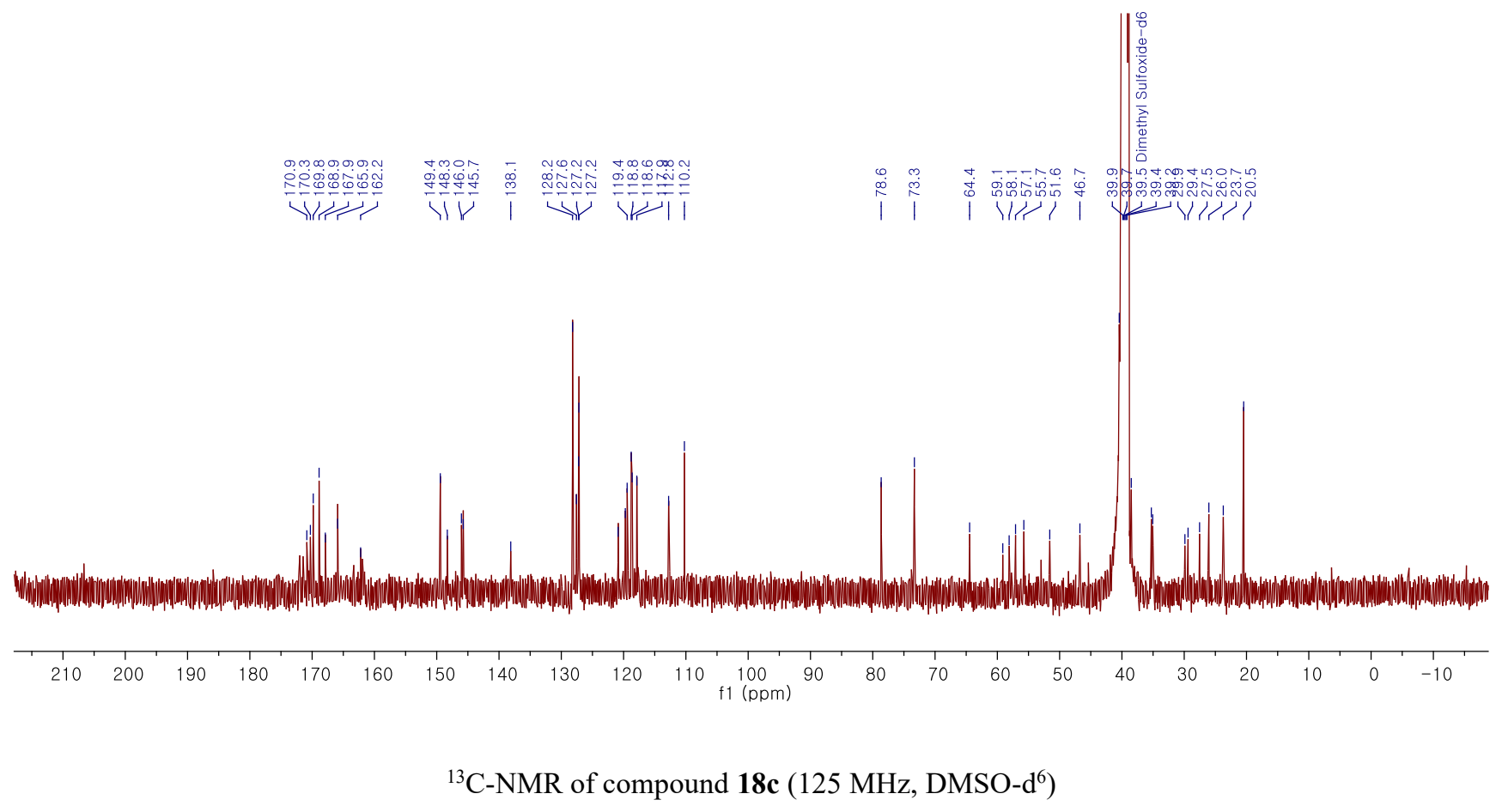




\section{Compound Spectrum SmartFormula Report}

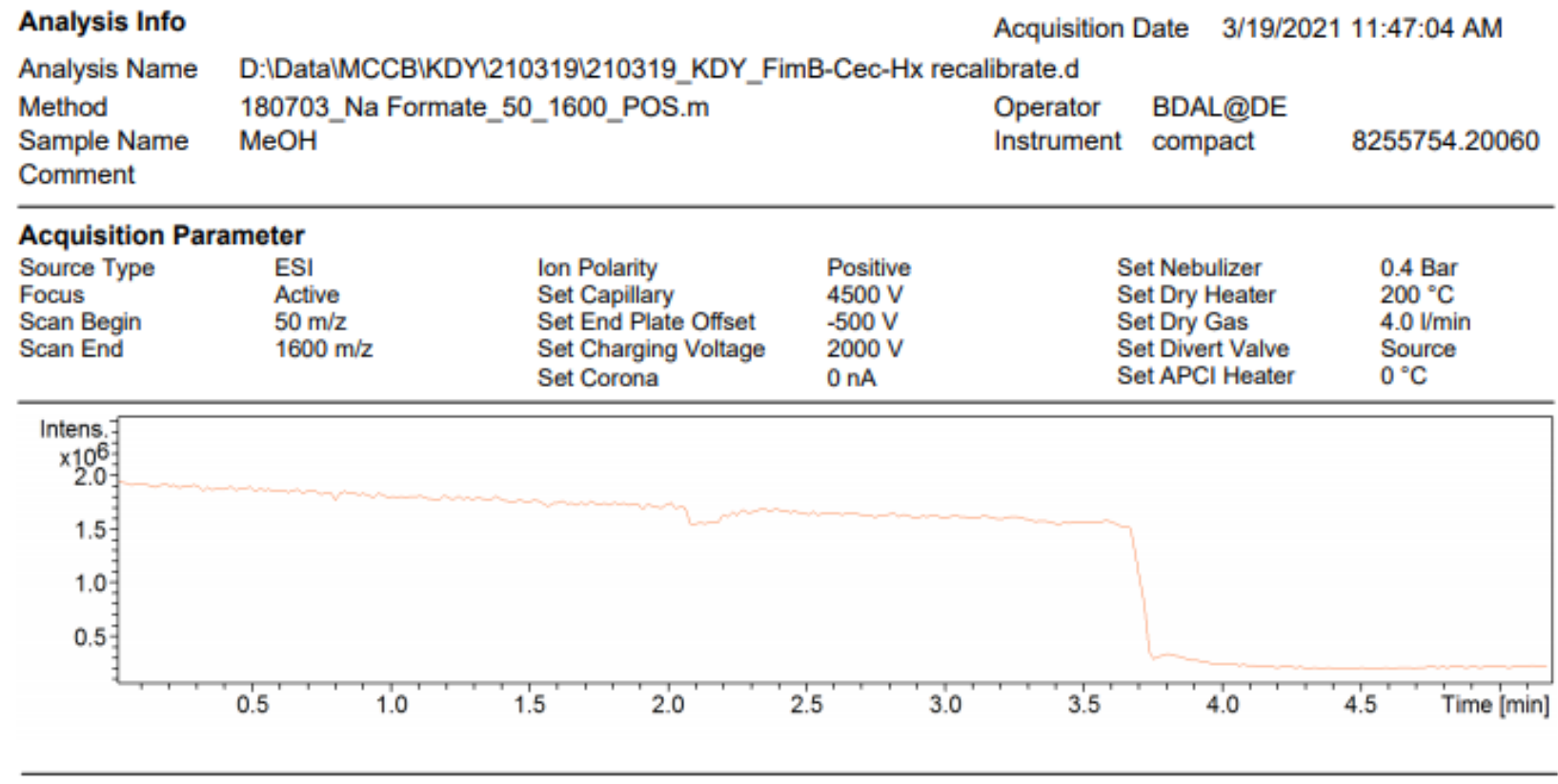

+MS, 5.0-5.1min \#295-296

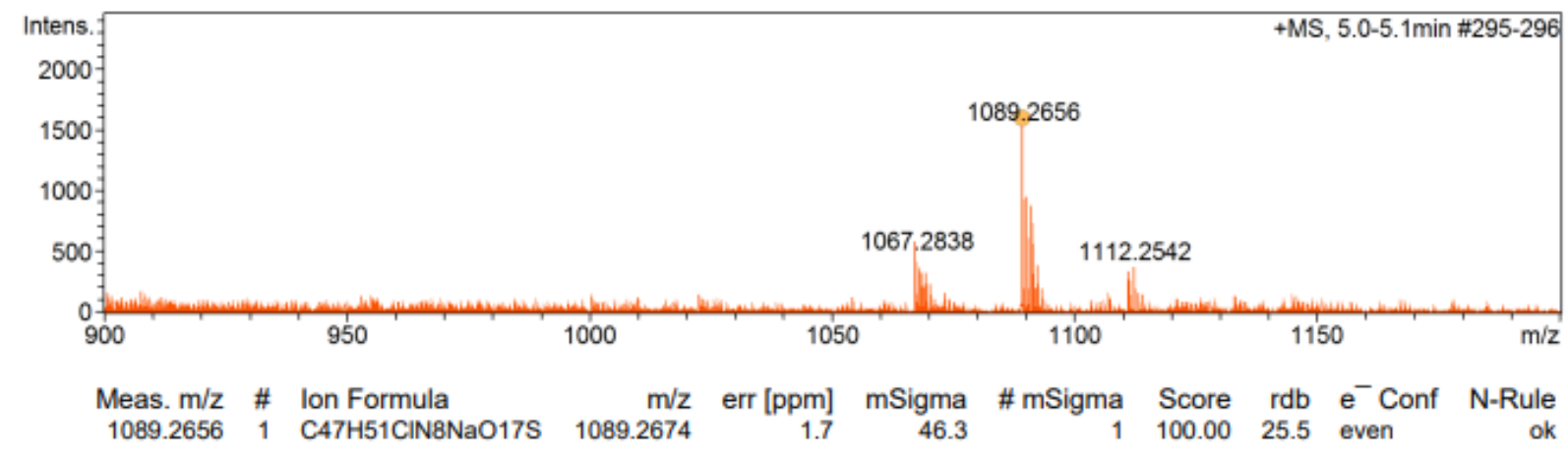

210319_KDY_FimB-Cec-Hx recalibrate.d

HR-MS of compound 18c 